\title{
Fuels from Microalgae: Technology Status, Potential, and Research Requirements
}

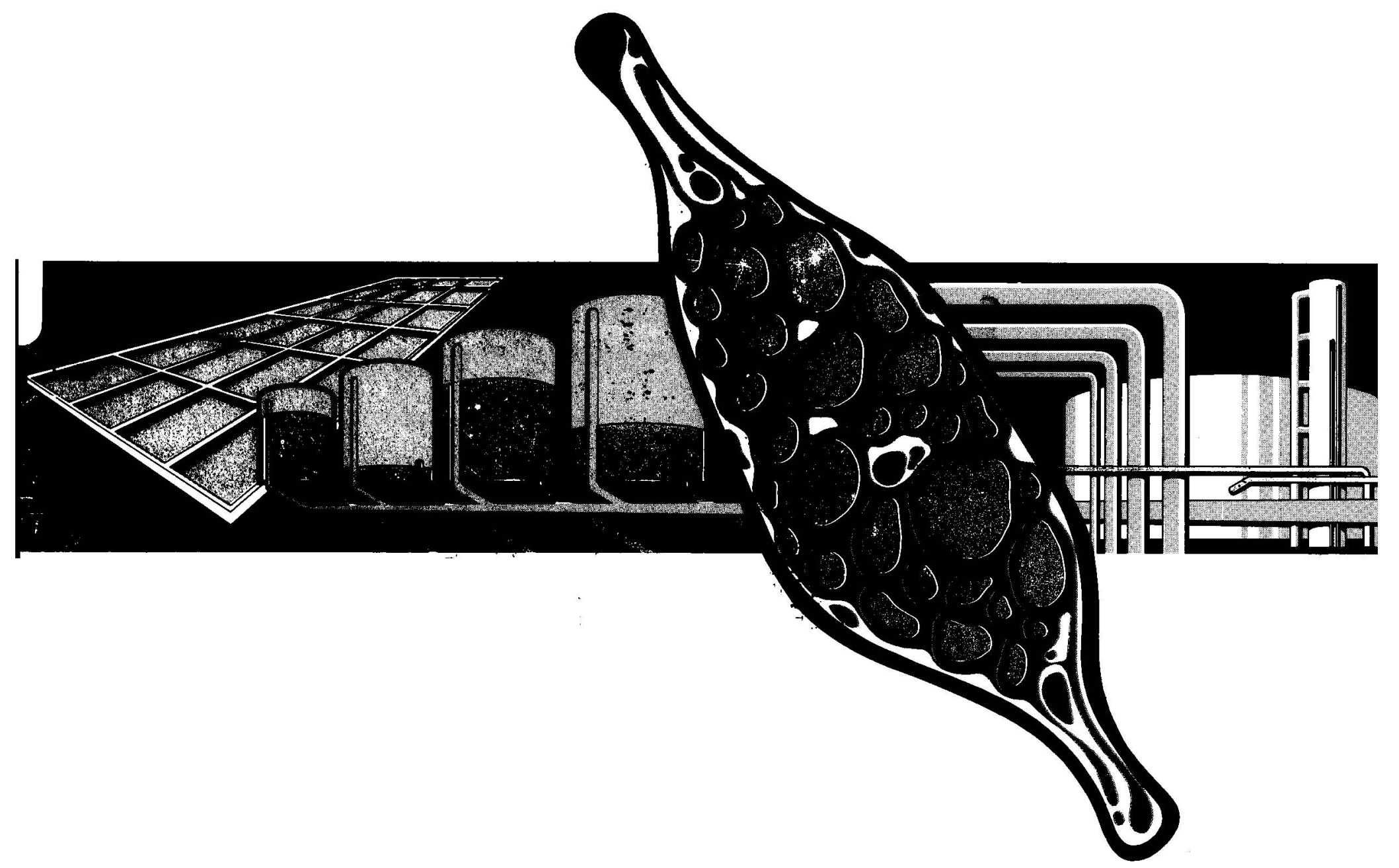




\section{NOTICE}

This report was prepared as an account of work sponsored by the United States Government. Neither the United States nor the United States Department of Energy, nor any of their employees, nor any of their contractors, subcontractors, or their employees, makes any warranty, expressed or implied, or assumes any legal liability or responsibility for the accuracy, completeness or usefulness of any information, apparatus, product or process disclosed, or represents that its use would not infringe privately owned rights.

Printed in the United States of America

Available from:

National Technical Information Service

U.S. Department of Commerce

5285 Port Royal Road

Springfield, VA 22161

Price: Microfiche A01

Printed Copy A08

Codes are used for pricing all publications. The code is determined by the number of pages in the publicalion. Information pertaining to the pricing codes can be found in the current issue of the following publicalions. which are generally available in most libraries: Energy Research Abstracts, (ERA): Govermmom! Roports Announcements and Index (GRA and 1): Scientific and Technical Abstract Roporls (STAR), ind publication. NTIS-PR-360 available from NTIS at the above address. 


\title{
Fuels from Microalgae: Technology Status, Potential, and Research Requirements
}

\author{
Bernie Neenan \\ Daniel Feinberg \\ Andrew Hill \\ Robins Mclntosh \\ Ken Terry
}

August 1986

Prepared under Task No. 4513.20

FTP No. 513

Solar Energy Research Institute

A Division of Midwest Research Institute

1617 Cole Boulevard

Golden, Colorado 80401-3393

Prepared for the

U.S. Department of Energy Contract No. DE-AC02-83CH10093 


\section{PREFACE}

This study was conducted at the request of the Department of Energy, Biomass Technology Division, to provide a comprehensive overview of the concept of fuels from microalgae. The scope of the study is limited to evaluating alternative fuels from mass cultured microalgae in the southwestern United States, the basis for current program activities. This approach focuses on the feasibility of producing fuel products from microalgae and does not specifically address application of the technology to other ends.

The authors acknowledge the contributions of Sid Browne and Art Power to the development and execution of this study. The strong and enlightened guidance of Sid Browne through the most demanding and formative stages of this project contributed immeasurably to the final report. Art Power provided insight and support to the engineering appraisals and resource evaluations critical to the overall technology evaluation. We also extend our appreciation to Stan Bull for his guidance, unwavering support, and considerable patience, and to Bill Barclay for timely review and technical guidance. Editorial and administrative assistance were generously provided by JoAnn Smith and Shirley Valdez.

Finally, an integral part of this assessment was the contribution of a dedicated and knowledgeable team of consultants who reviewed the input parameters, provided insight on the complexities of algal culture, and pointed out issues that otherwise might have gone unnoticed. The authors, while retaining responsibility for the content of the report, acknowledge the contributions of D. Brune, J. Dodd, G. Eskamani, K. Foster, C. Hinman, J. Huguenin, R. Krauss, T. Mumford, E. Pryde, and R. Young.

Approved for

Solar Energy Research Institute

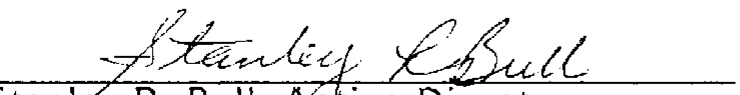

Stanley R. Bull, Acting Director

Solar Fuels Research Division 


\section{EXECUTIVE SUMMARY}

This technology assessment demonstrates that gasolime and denel fuels could be produced from mass-cultured microalgae at prices that will be innpelitive with conventional

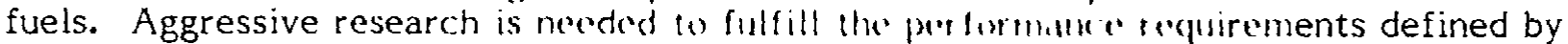
the analysis, but the required improvements are whthin he bouncts of attainability and have been closely approached under controlled conditums. lumprovements need to be made in the productivity, lipid yield, and salmity and temperature tolerance of microalgae species. Engineering improvements are also needed in the cultivation system design and in harvesting. Two critical resource parameters are the availability of saline waters in the Southwest deserts and the cost of carbon dioxide. Based on the achievement of these research goals and economic availability of the critical resources, liquid fuels that are potential direct substitutes for conventional hydrocarbon fuels can be produced from microalgae for $\$ 1.60-\$ 2.00 /$ gallon by the year 2010 .

In 1979, DOE and SERI initiated a research program to pursue opportunities for producing liquid fuels from microalgae. Microalgae are unique photosynthetic organisms in that they are known to accumulate storage lipids in great quantities and will thrive in high salinity water. The DOE/SERI program emphasizes the development of microalgae systems in the desert Southwest because this area offers flat land, high incident solar radiation, few competing land uses, and large reservoirs of saline water. Thus, locating the mass culture facility in this region minimizes land costs, while the use of saline water, not suitable for agricultural, domestic, or industrial purposes, minimizes competition with other uses for the limited supplies of fresh water in the Southwest. Finally, the program is focused on the production of lipids from microalgae because plant storage lipids could be among the best biomass feedstocks for producing renewable, high-energy liquid fuels such as gasoline and diesel fuel.

To assess the feasibility of producing fuels from microalgae, four major questions were answered in this evaluation.

1. Which fuel products are the best candidates for production from microalgae?

2. Given those fuel products, what improvements in the microalgae cultivation systems are required to achieve greater cost effectiveness?

3. Given the microalgae feedstock characteristics, what improvements in fuel processing are required to achieve cost effectiveness?

4. Given the required improvements in the mass culture system and fuel processing, what are the implications for a research program designed to meet the objective?

Analysis of a number of fuel conversion options for microalgal biomass has demonstrated that the promise of microalgae for fuel production is best realized through the utilization of conversion processes based on cellular lipids, an energy-rich hydrocarbon. The ability to produce lipids that can constitute $60 \%$ or more of the total biomass is a distinguishing characteristic of microalgae and makes them uniquely attractive candidates for conversion to liquid fuels. The two most promising fuel conversion options are transesterification to produce fuels similar to diesel fuels and catalytic conversion to produce gasoline. While microalgal lipids represent the premium energy product, the energy trapped in the other biomass constituents can also be utilized; e.g., the cell residue after lipid extraction can be anaerobically digested for the production of methane and carbon dioxide. 
The availability of saline water will be an important factor in determining the ultimate scale of fuel production technology based on this resource. Due to high evaporation rates, water demands for uncovered cultures in this region will be extremely high. While saline aquifers are found throughout the Southwest, the total volume these aquifers can supply on a sustained basis has not been determined. Since very little information is available on the saline water resources of the desert Southwest, the quantification of this resource is a high priority activity.

Carbon dioxide supply is the largest single contributor to the cost of liquid fuels derived from microalgae. However, the acquisition of sufficient quantities of carbon dioxide should not impose constraints on the ultimate scale of the microalgal fuels technology. Existing and proposed coal-fired power plants will produce carbon dioxide in excess of the quantity required for microalgal production. Competing demands from enhanced oil recovery (EOR) are not anticipated, since most oil fields in the area are expected to be depleted by the year 2010, which is the expected date of emergence of an extensive microalgal mass culture technology for liquid fuel production. If methods were developed for the recovery of carbon dioxide previously injected into oil wells, these large reservoirs could supply additional abundant quantities of low-cost carbon dioxide.

The major issues to be resolved in the mass culture technology are biological. For this technology to become cost competitive, the biological productivity of these systems must be improved. The production analysis indicates that photosynthetic efficiencies of $12 \%-16 \%$ must be attained, and that $50 \%-60 \%$ by weight of the biomass produced must be in the form of lipids. If higher lipid contents were achieved, lower photosynthetic efficiency would be acceptable, and vice versa.

In addition to displaying improved productivity, species must demonstrate environmental tolerance characteristics that make them suitable for outdoor culture in arid regions. The productivity analysis has identified species salinity tolerance as a particularly significant aspect of environmental tolerance, since operation of cultures at high salinities is necessary to control water requirements through minimization of water consumption for blowdown.

The basic engineering needs for microalgal culture have been identified and form the basis of a significant portion of the production analysis. Considerable additional engineering research and development will be required. Important engineering issues are: efficient and inexpensive harvesting methods, mechanisms to reduce evaporation, production system design, brine disposal, and carbon dioxide input systems. 


\section{TABLE OF CONTINTS}

Page

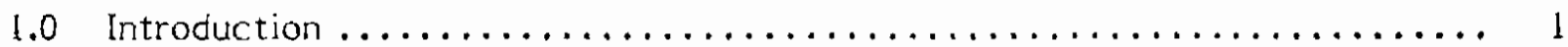

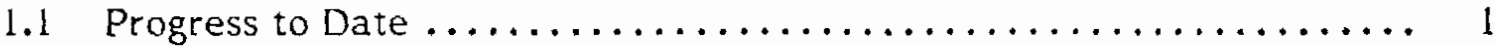

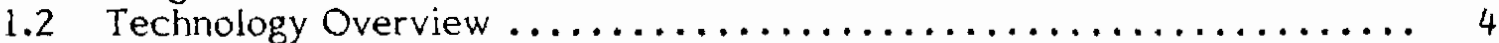

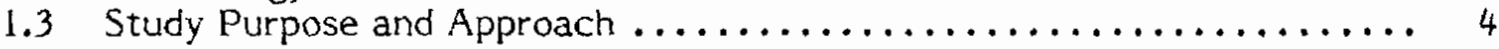

2.0 Principles of Microalgal Fuels Technology $\ldots \ldots \ldots \ldots \ldots \ldots \ldots \ldots \ldots \ldots \ldots$. 8

2.1 Mass Culture Technology $\ldots \ldots \ldots \ldots \ldots \ldots \ldots \ldots \ldots \ldots \ldots \ldots \ldots$

2.1.1 Introduction ................................ 8

2.1.2 Culture Water Demand and Salt Balance............... 8

2.1.3 Carbon Dioxide Supply .......................... 11

$2.1 .4 \quad$ Nutrient Supply ............................. 12

2.1.5 Algal Species for Mass Culture ................... 14

2.2 Design of Mass Culture Production Systems ................. 16

2.2 .1 Growth Conditions .......................... 16

2.2.2 Light Utilization in Mass Cultures ................. 19

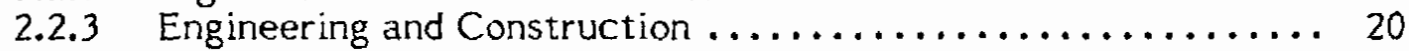

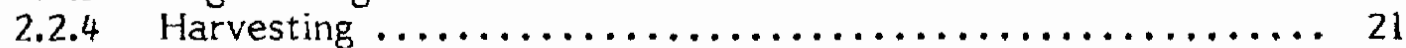

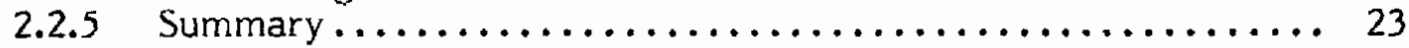

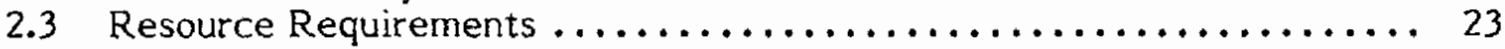

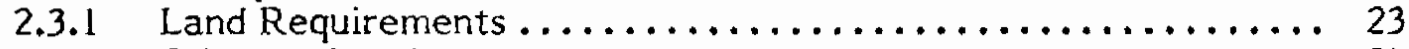

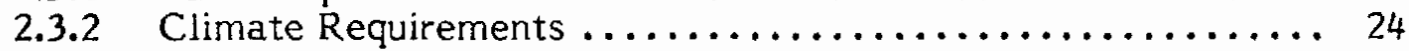

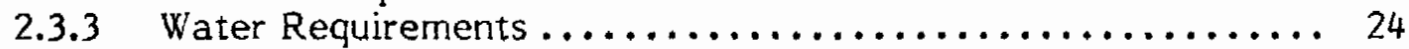

2.3.4 Carbon Dioxide Supply and Demand ................ 25

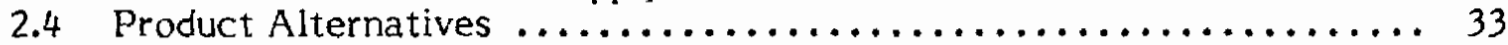

2.4.1 Functional Properties ......................... 33

2.4.2 Overview of Fuel Product Network ................ 35

2.4.3 General Characteristics of Crude Microalgae Feedstock ...... 35

2.4.4 Fuel Products from Lipids ..................... 41

2.4.5 Other Products and Processes .................... 45

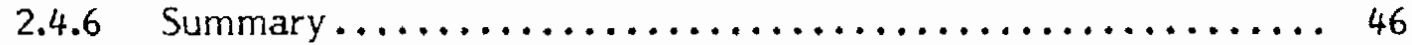

3.0 Mass Cuiture Economics $\ldots \ldots \ldots \ldots \ldots \ldots \ldots \ldots \ldots \ldots \ldots \ldots \ldots \ldots \ldots$

3.1 Process Design Economics ......................... 48

3.1.1 Pond Configurations and Cost Factors .............. 48

3.1.2 Harvesting Options .......................... 49

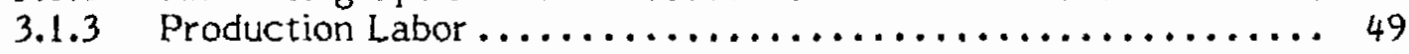

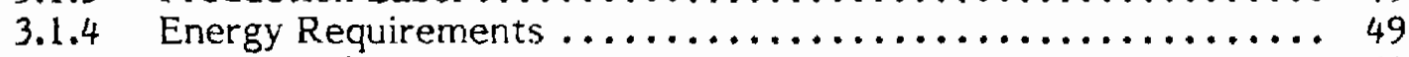

3.1.5 Resource Supplies .......................... 50 


\section{TABLE OF CONTENTS (continued)}

3.2 Analytical Method for Evaluation ..................... 51

3.2.1 Model Description ........................... 51

3.2.2 Overview of Reference Values and Ranges for Model

Parameters............................. 51

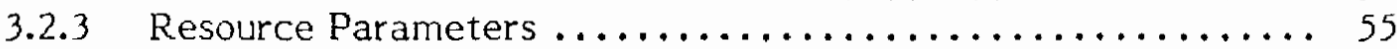

3.2.4 Facility Design Parameters .................... 59

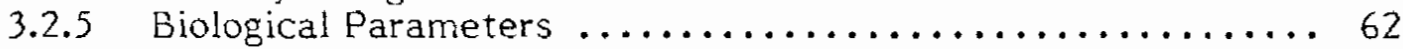

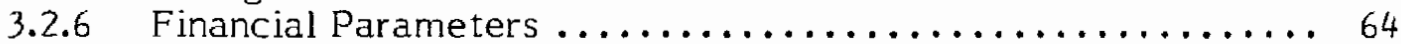

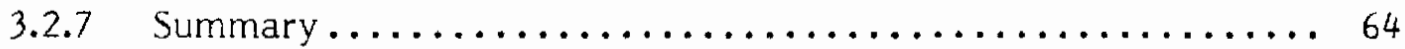

3.3 Reference Case Economics .......................... 64

3.3.1 Cost Contribution to Reference Case ................. 65

3.3.2 Summary.............................. 69

4.0 Economics of Producing Fuels from Microalgae $\ldots \ldots \ldots \ldots \ldots \ldots \ldots \ldots \ldots$. 1

4.1 Estimating Algal Processing Costs $\ldots \ldots \ldots \ldots \ldots \ldots \ldots \ldots \ldots \ldots \ldots \ldots$

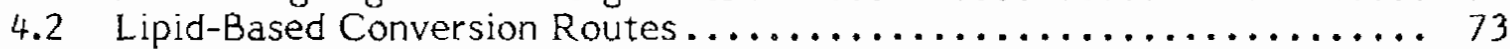

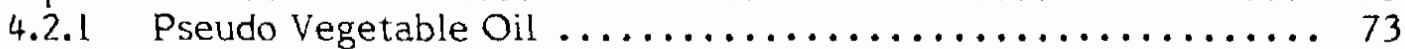

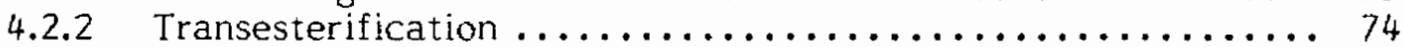

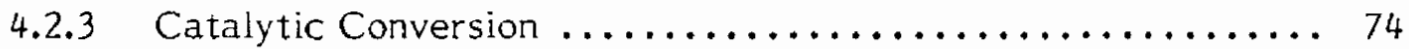

4.3 Nonlipid-Based Processes .................................. 74

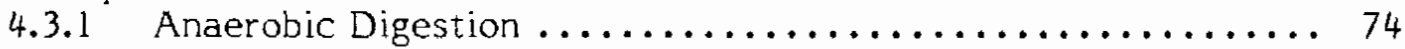

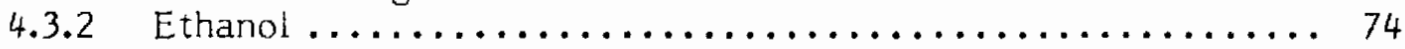

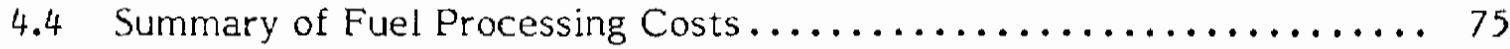

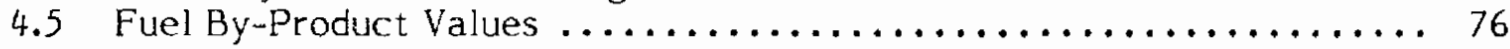

4.6 Reference Fuel Production Costs $\ldots \ldots \ldots \ldots \ldots \ldots \ldots \ldots \ldots \ldots$

5.0 Initial Screening of Cost Contributions $\ldots \ldots \ldots \ldots \ldots \ldots \ldots \ldots \ldots \ldots$

5.1 Resource Parameter Sensitivity ..................... 86

5.2 Facility Design Parameter Sensitivity ................... 87

5.3 Biology Parameter Sensitivity ........................ 90

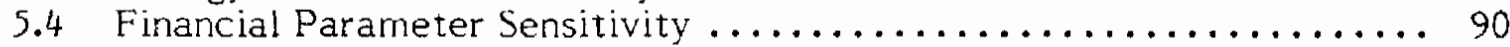

5.5 Important Parameters for Further Evaluations ............... 93

6.0 Evaluation of Important Technology Issues .................... 95

6.1 Important Linkages and Critical Issues in Microalgae

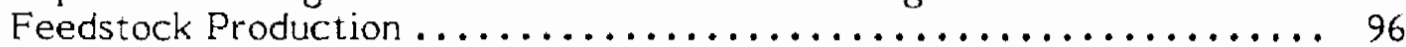

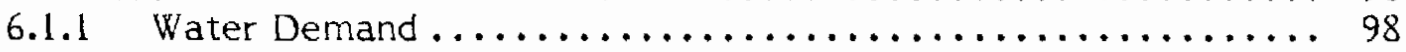

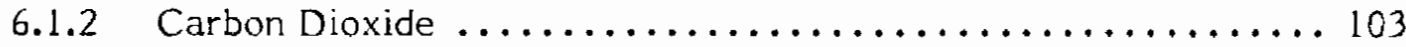

6.1 .3 Algal Productivity and Products .................... 107

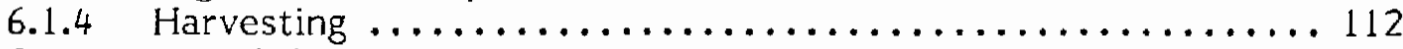

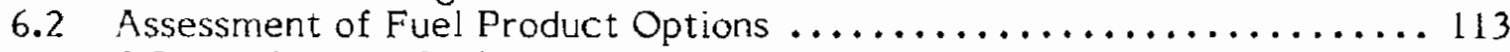

6.2 .1 Process Options . .............................113

6.2 .2 Screening of Fuel Option Feasibility $\ldots \ldots \ldots \ldots \ldots \ldots \ldots \ldots 113$ 
TABLE OF CONTENTS (concluded)

$\underline{\text { Page }}$

6.3 Attainability Targets for Microalgae Feedstock Production

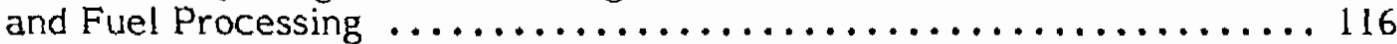
6.3.1 An Attainable Production Technology Configuration ......... 117

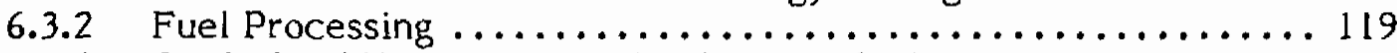
6.3.3 Analysis of Feedstock Production and Fuel Processing Requirements................................. 123

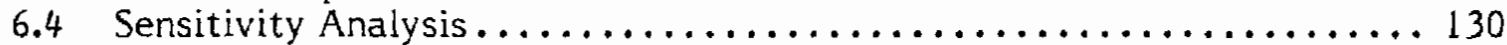
6.4.1 Sensitivity of the Attainability Production Cost

to Constraints .................................. 130 6.4.2 Special Attainability Cases with Low Production Costs ....... 135 6.4 .3 Lipid Sensitivity Cases $\ldots \ldots \ldots \ldots \ldots \ldots \ldots \ldots \ldots \ldots \ldots \ldots \ldots$

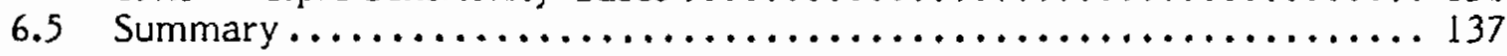

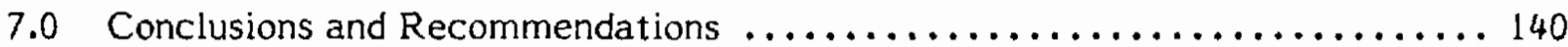

7.1 Fuel Products from Microalgal Feedstocks ................... 140

7.2 Resources and Environmental Considerations .................. 141

7.3 Biological and Engineering Aspects of Feedstock Production ........ 142

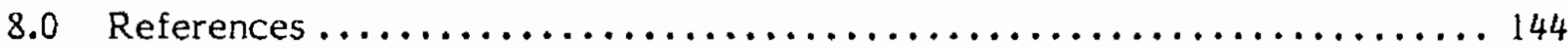

Appendix A Microalgae Production Model ......................... 149 


\section{LIST OF FIGURES}

2-1 Major $\mathrm{CO}_{2}$ Productive Areas and Oil Reservoirs Amenable

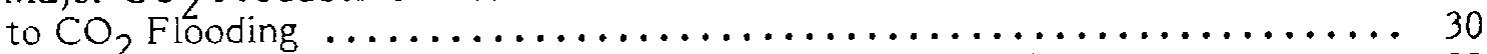

2-2 Generalized Overall Refinery from Crude Oil to Salable Products ........ 38

2-3 Network of Fuel Products from Natural Gas . . . . . . . . . . . . . . 39

2-4 Network of Products from Coal ........................ 40

2-5 Major Lipid Fractions as Recovered via Sequential

Solvent Extraction .............................. 43

2-6 Product Distribution from Catalytic Conversion of Triglycerides ......... 44

3-1 Overview of Algal Production and Economic Model (APEM) ......... 52

3-2 Average Annual Net Evaporation from Pan A Data ................ 56

3-3 Contribution to Product Cost by General Cost Category ............ 66

3-4 Cost Contribution Detail for Direct Operating and

Maintenance Expenses ............................. 66

3-5 General Cost Contribution of Capital Cost Categories ............... 68

3-6 Cost Contribution Detail for Nondepreciable Portion of

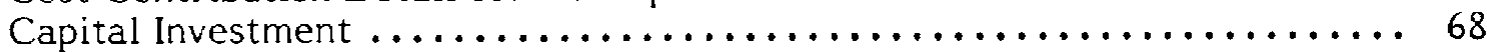

3-7 Cost Contribution Detail for Depreciable Capital Investment .......... 69

4-1 Overview of Microalgae Fuel Refining $\ldots \ldots \ldots \ldots \ldots \ldots \ldots \ldots \ldots \ldots \ldots \ldots$

4-2 Pseudo-Vegetable Oil Production Process Generalized Flow Diagram . . . . . . 73

4-3 Schematic of Transesterification Process $\ldots \ldots \ldots \ldots \ldots \ldots \ldots \ldots \ldots \ldots \ldots$

4-4 Mobil M-gas Process Adapted for Lipid Oils . . . . . . . . . . . . . . . 77

4-5 Anaerobic Digestion of Microalgae $\ldots \ldots \ldots \ldots \ldots \ldots \ldots \ldots \ldots \ldots \ldots \ldots$

4-6 Ethanol Production Process Generalized Flow Diagram . . . . . . . . . . . . 79

4-7 Cost Contribution Detail for Lipid-Based Processes ............... 81

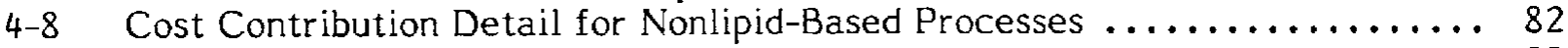

5-1 Resource Parameter Sensitivity ......................... 88

5-2 Facility Design Parameter Sensitivities ....................... 89

5-3 Biological Parameter Sensitivities ......................... 91

5-4 Sensitivity of Algal Production Cost to Detention Time, Depth,

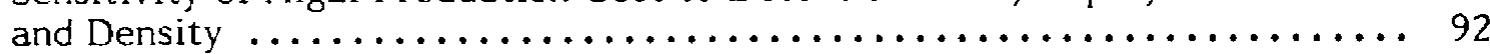

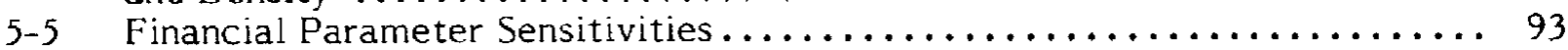

6-1 Linkages among Water Source Salinity, Water Use, and Algal Species Requirements............................... 99

6-2 Sensitivity of Reference Production Cost to Variations in the Evaporation Rate and Algal Salinity Tolerance ................. 100

6-3 Carbon Dioxide Costs and Transportation Distance for Reference ........ 104

6-4 Carbon Dioxide Scale Economies for Reference Transportation .......... 104

6-5 Experimental Design for Evaluation of Algal Species Improvements ....... 109

6-6 Biomass Production Cost Sensitivity to Photosynthetic Efficiency and Lipid Content ................................... 11

6-7 Reference, Research Opportunity, and Fuel Option Cases and

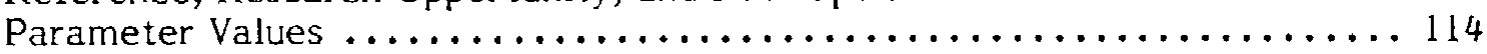

6-8 Capital Costs for Attainability Fuel Processing Plants .............. 123

6-9 Total Operating Costs for Attainability Fuel Processing Plants ......... 124

6-10 Operating Costs (Excluding Feedstock) for Attainability Fuel

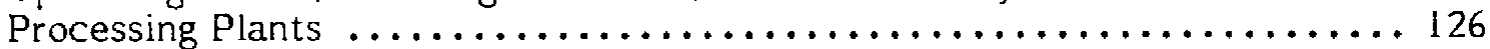

6-11 Photosynthetic Efficiencies Required to Maintain Fuel Product Costs Equal to the Cost Goals: Uncertainties that Increase Costs . . . . . . . . 134 


\section{LIST OF FIGURES (concluded)}

6-12 Photosynthetic Efficiencies Required to Maintain Fuel Product Costs Equal to the Cost Goals: Uncertainties that Decrease Costs . . . . . . . . 138

6-13 Combinations of Lipid Content and Photosynthetic Efficiency that Will Lead to the Production of Cost-Competitive Liquid Fuels . . . . . . . . . . . 139 


\section{LIST OF TABLES}

Page

2-1 Potential $\mathrm{CO}_{2}$ Demand by Petroleum Basin $\ldots \ldots \ldots \ldots \ldots \ldots \ldots \ldots \ldots \ldots \ldots$

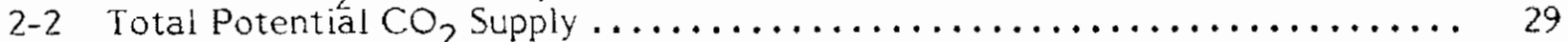

2-3 Carbon Dioxide Supplies in the Permian Basin $\ldots \ldots \ldots \ldots \ldots \ldots \ldots \ldots \ldots \ldots \ldots$

2-4 Energy Content of Some Liquid Fuel Products .................... 34

2-5 Composition and Energy Content of a Representative Sample of

Crude Algae Feedstock ............................. 41

2-6 Suggested Standards for Vegetable Oil and Ester Fuels vs. Diesel Oil Specifications .......................... 42

2-7 Summary of Reference Processing Product Yields $\ldots \ldots \ldots \ldots \ldots \ldots \ldots \ldots \ldots 47$

3-1 Parameter Ranges Identified for Microalgae Systems ................ 53

3-2 Summary of Reference Production Facility Cost Contributions

for Annual Direct Cost and Capital Cost ................... 70

4-1 Reference Production Summary for Microalgae Processing Options......... 75

4-2 Summary of Reference Capital, Operating, and Allowable Feedstock

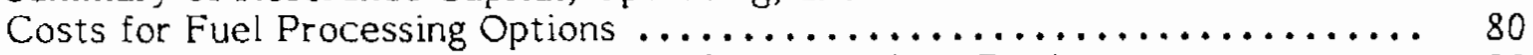

4-3 Estimated Year 2000 Fuel Cost Goals for Microalgae Products . . . . . . . . 83

4-4 Algal Fuel Processing By-products and Values ................... 83

4-5 Comparison of Feedstock Production Cost and Allowable

Processing Cost for the Reference Case .................... 85

5-1 Summary of Most Critical Resource, Facility Design, and

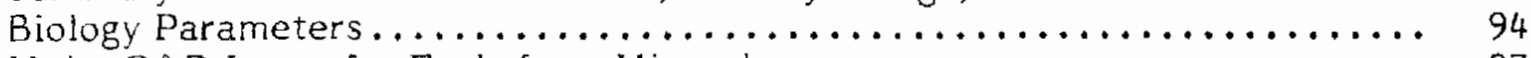

6-1 Major R\&D Issues for Fuels from Microalgae .................... 97

6-2 Tradeoffs between Salinity Tolerance and Water Costs ............. 102

6-3 Summary of Alternative Scenarios to Reduce $\mathrm{CO}_{2}$ Costs to a Mass Culture Facility ........................... 106

6-4 Parameter Assumptions for Evaluating Photosynthesis on Lipid Content ................................... 108

6-5 Comparison of Reference Algal Feedstock Values and Attainable Levels

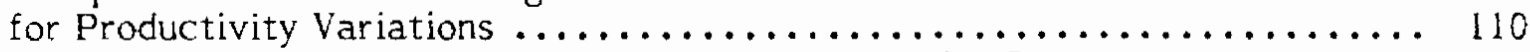

6-6 Productivity and Fuel Type Cases for Algal Processing Evaluation ........ 116

6-7 Parameter Levels for Attainability Microalgae Production Cases:

Changes from Reference Case ......................... 119

6-8 Summary Output for Attainability Microalgae Production Cases . . . . . . . . 120

6-9 Reference Case and Attainability Values for Lipid Processing Plant

Scale and Conversion Efficiency .......................... 121

6-10 Summary of Attainability Product Yields $\ldots \ldots \ldots \ldots \ldots \ldots \ldots \ldots \ldots \ldots \ldots \ldots . \ldots \ldots$

6-11 Production Summary of Attainability Microalgae Refinery Options ... . . . . . 122

6-12 Summary of Attainability Capital and Operating Costs for Fuel

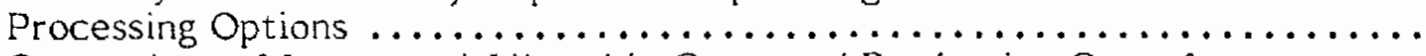

6-13 Comparison of Integrated Allowable Costs and Production Costs for Microalgae-Derived Fuels . . ...........................

6-14 Derived Microalgae Fuel Production Costs for Integrated

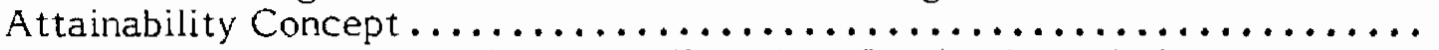

6-15 Parameter Values for Attainability Microalgae Production: Variances from Reference Case Values ........................... 
LIST OF TABLES (concluded)

$\underline{\text { Page }}$

6-16 Input and Output Material Flows for the Integrated Attainability

Microalgae Production Concept .......................... 130

6-17 Summary of Integrated Attainability Production Facility

Cost Contributions ................................. 131

6-18 Sensitivity Cases for Attainable Microalgae Production

Cost: Uncertainties that Increase Costs ...................... 132

6-19 Mitigating PSE Levels for Attainability Production

Case Sensitivities ................................. 133

6-20 Sensitivity Cases for Attainable Microalgae Production Cost:

Uncertainties that Decrease Costs ........................ 136 


\subsection{INTRODUCTION}

Microalgae are single-celled aquatic plants that have many potential uses including irrigation, waste-water treatment, and the production of food, fuels, and commodity chemicals. The SERI/DOE microalgae program focuses on the production of fuels from microalgae feedstocks. This study presents the results of a comprehensive evaluation that assesses the technology of fuels from microalgae and its potential for becoming a costeffective energy supply option.

\subsection{Progress to Date}

The concept of using mass-cultured algae for fuel production grew out of earlier efforts to use algae for food production and waste treatment (Burlew 1953). Some of these efforts have produced algae with a high market value. For example, the blue-green alga Spirulina produces high-quality protein and is sold in health food stores for up to $\$ 10 / \mathrm{lb}$. Researchers at the Carnegie Institute (Burlew 1953) experimented with mass culture of algae for food in the early 1950s, and this work led to expanded efforts by American, German, Israeli, and other researchers to mass-produce various species of microalgae for both waste water treatment and animal feed protein (Shelef and Soeder 1980).

The use of microalgae as a fuel was first proposed 30 years ago for the production of methane gas (Meier 1955). In the 1970s, the cost of conventional fuels began rising rapidly, and the possibility of using algae as a fuel source received renewed attention.

In 1979, DOE and SERI initiated a research program to pursue opportunities for producing fuels from microalgae. Although previous research on algae grown for other uses provided a basic foundation for the program, the unique challenges of fuel production required a directed, goal-oriented research and development program.

Past research and assessment efforts have resulted in a concept for using microalgae that has four main features: (1) a mass culture system for producing microalgae feedstocks, (2) location of mass culture facilities in the U.S. desert Southwest, (3) the use of saline waters, and (4) an emphasis on the production of lipids. Mass culture of microalgae is important to the viability of the technology because it would produce feedstocks in sufficient quantities to take advantage of economies of scale in fuel processing.

The DOE/SERI program emphasizes the development of microalgae systems in the desert Southwest because this area offers flat land, high incident solar radiation, and few competing land uses; locating the mass culture facility in this region would minimize land costs. The use of saline water, which is a suitable medium for the growth of many microalgae, minimizes both water costs and competition with other uses for limited supplies of fresh water in the Southwest. Finally, the program emphasizes the production of lipids from microalgae because plant storage lipids could be among the best biomass feedstocks for producing renewable, high-energy liquid fuels. Microalgae are among the few photosynthetic organisms that directly produce and are known to accumulate storage lipids in great quantities. 


\section{Microalgae Mass Culture Technology}

Mass culture technology requires several components that must be integrated into a system for efficient production and harvesting.
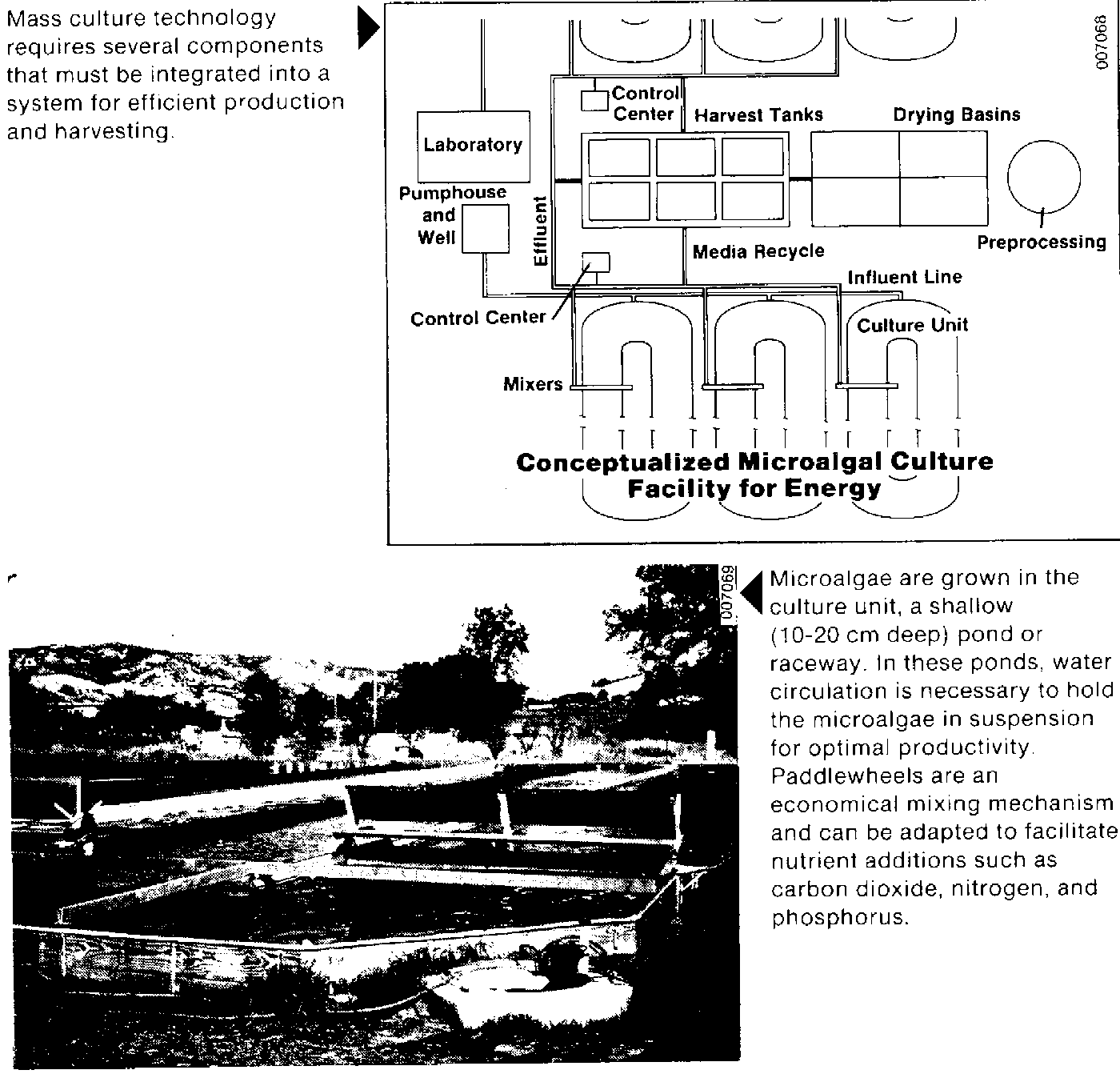

Microalgae are grown in the culture unit, a shallow (10-20 cm deep) pond or raceway. In these ponds, water circulation is necessary to hold the microalgae in suspension for optimal productivity Paddlewheels are an economical mixing mechanism and can be adapted to facilitate nutrient additions such as carbon dioxide, nitrogen, and phosphorus.
High productivity requires uniform mixing of the culture to assure that the algae circulate systematically between the dark bottom zones and the lighted surface zones. Foils have been proposed to provide this uniform mixing.

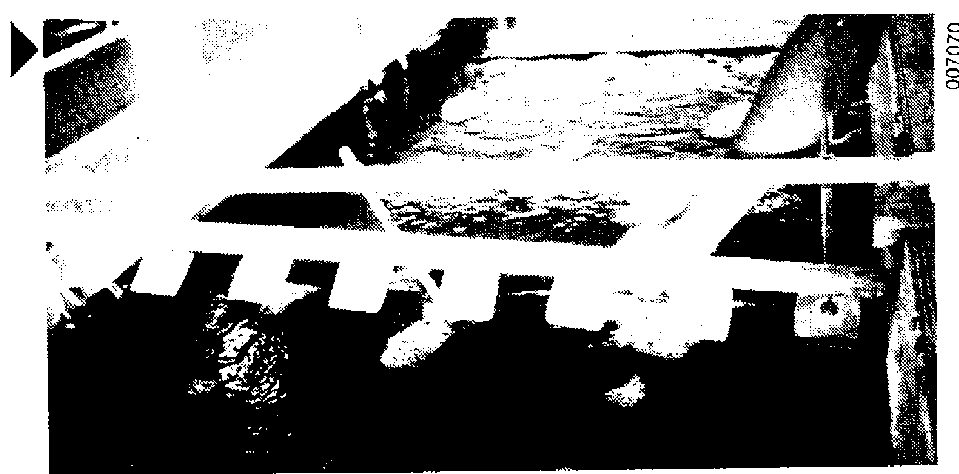


Microalgae Mass Culture Technology

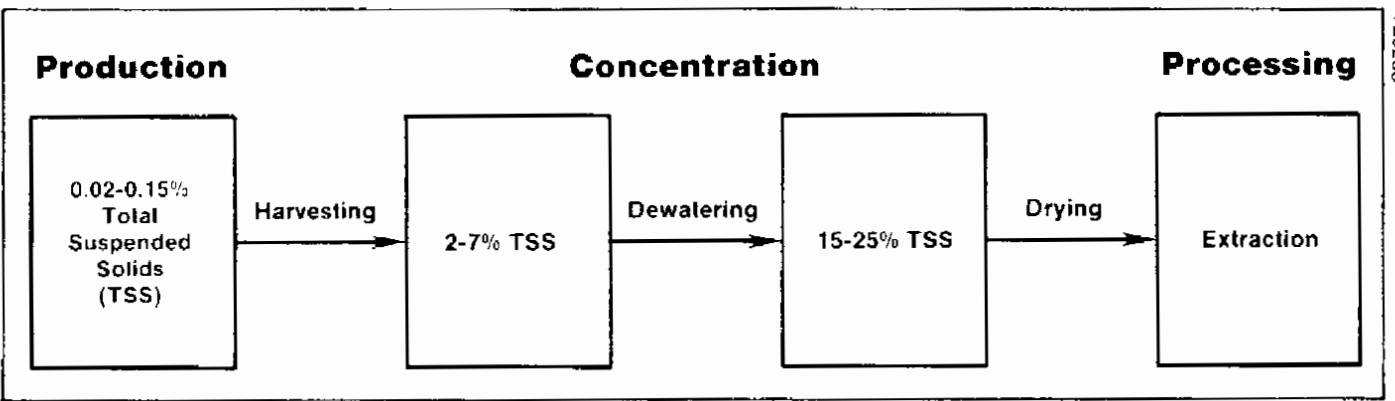

Microalgae must be harvested from the culture medium. concentrated, and dried before they can be processed. The optimal harvesting technique depends on the characteristics of the species. Some algae can be induced to form clumps (autoflocculate) and float or settle to facilitate harvesting Otherwise filtering must be employed to harvest the small algal cells.
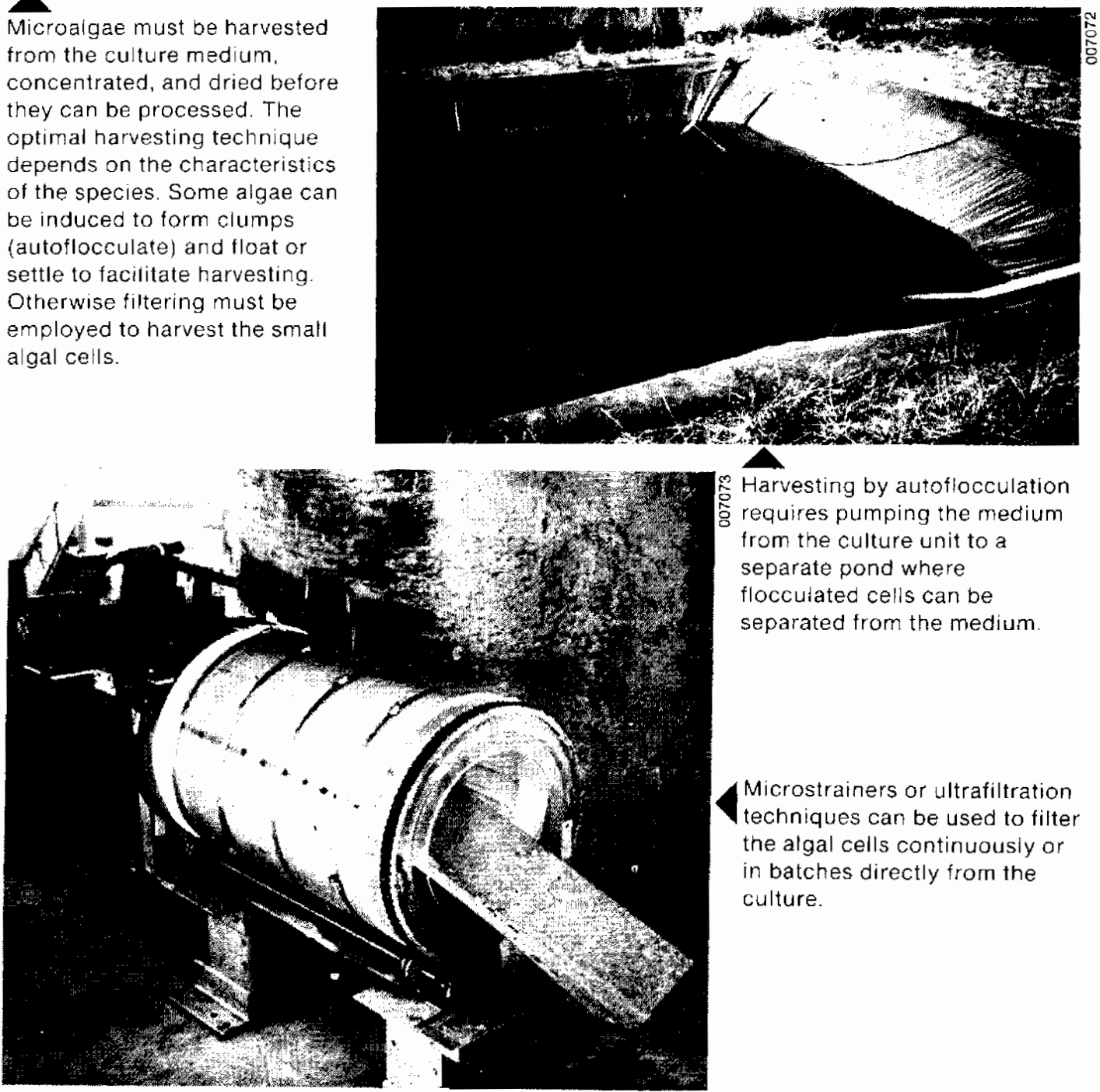

Harvesting by autoflocculation requires pumping the medium from the culture unit to a separate pond where flocculated cells can be separated from the medium

Microstrainers or ultrafiltration techniques can be used to filter the algal cells continuously or in batches directly from the culture. 
In research sponsored by the SERI/DOE microalgae program and other, independent researchers, sustained photosynthetic efficiency* in the laboratory and controlled outdoor experiments has achieved over $12 \%$, and lipid concentrations, normally about $10 \%-$ $20 \%$, have reached $60 \%$ using nitrogen starvation techniques. These are significant achievements that represent a valuable beginning to an emerging technology. However, progress must be measured against the ultimate goal of producing cost-competitive fuels. The objective of this study is to define research goals consistent with costcompetitiveness.

\subsection{Technology Overview}

Two basic steps are involved in producing fuels from microalgae: feedstock production and fuel processing. As the technology is envisioned today, microalgae would be grown in large, shallow ponds of saline water in the desert Southwest, harvested, and processed to make a feedstock that can be used in the production of liquid or gaseous fuels. This specific concept is the subject of this evaluation.**

Two characteristics of microalgal fuels technologies make them particularly difficult to assess. First, a wide variety of fuels can be produced, including ethanol, triglyceridebased diesel fuel, ester fuel, methane, and gasoline. Each fuel has a different market value as well as different feedstock and fuel processing requirements. It is not clear which fuels are the best candidates for production from microalgae.

The technology's second major feature is that it contains interrelationships so complex that no single parameter--either within or between phases of production--can be evaluated or optimized in isolation. The magnitude of the assessment problem is illustrated by the following example.

To improve the cost effectiveness of microalgae-based fuels, the productivity of the mass culture system must be improved from current levels. But specific improvements-in photosynthetic efficiency, nutrient supplies, and the production of different algal products--cannot be evaluated completely without first specifying the fuel product to be produced and its processing requirements. Similarly, the required improvements in fuel processing cannot be determined without first specifying the feedstock to be processed and the fuel product to be produced. Finally, the most promising fuel products cannot be identified without an understanding of feedstock characteristics and fuel processing requirements. These complex technology interdependencies are described in detail in Section 2.0 and largely determined this study's assessment approach.

\subsection{Study Purpose and Approach}

The broad purpose of this study is to assess the feasibility of producing fuels from microalgae. Specifically, achieving this goal involves answering four major questions. First, which fuel products are the best candidates for production from microalgae? Second,

* In this document photosynthetic efficiency is defined in terms of photosynthetically active radiation (PAR), those wavelengths of sunlight between 400 and $700 \mathrm{~nm}$.

**A comprehensive overview of algal biomass technologies can be found in Barclay and McIntosh (1986). 
given those fuel products, what improvements in the feedstock production process are required to achieve greater cost effectiveness? Third, given the microalgae feedstock characteristics, what improvements in fuel processing are required to achieve cost effectiveness? Finally, given the required improvements in the mass culture system and fuel processing, what are the implications for a research program designed to meet the objective?

Because of the complex interrelationships within and between feedstock production and fuel processing, it is not possible to answer these questions in sequential order. Instead, the nature of the technology requires an iterative approach that allows for the sequential screening of major technical issues. Generally, in our assessment process we established a reference case for mass culture and fuel processing, conducted sensitivity analyses to identify major cost drivers and optimal fuel products, and then established cost and performance requirements for a viable microalgal fuels technology. Specifically, the assessment approach involves four basic steps.

1. Estimate current costs for feedstock production. This step is necessary to provide a starting point from which the analysis could proceed. Section 3.0 provides an overview of the processes involved in mass culture production of microalgae feedstocks, derives representative characteristics for each step in the production process, and then estimates the cost of producing feedstock from the reference system. The reference case is based on the best available information from small- and large-scale experiments and engineering studies. Thus, it represents a first approximation of the expected costs if a production facility were constructed and operated today and identifies ranges for all variables that represent the potential for improvement.

2. Estimate current costs for fuel processing. This step provides a basis for further evaluation. In Section 4.0 we use the feedstock characteristics derived in Section 2.0 to establish a reference fuel conversion process and then estimate the cost of fuel processing. As in the feedstock reference system, the fuel processing reference case is based on best available information and represents a first approximation of processing technology.

3. Determine the major parameters affecting the cost of microalgae feedstocks. More than 100 parameters were specified in the development of the reference mass culture system. This step was necessary to screen the most important parameters so that later analyses could be reduced to manageable proportions. Section 5.0 describes the sensitivity analyses that were conducted to identify the major cost drivers.

4. Assess $R \& D$ requirements for both feedstock production and fuel processing. This step simultaneously permits an assessment of the viability of the technology and helps to define R\&D priorities. Section 6.0 begins by assessing important linkages among related parameters in mass culture technology. These linkages reduce the major cost drivers to their lowest common denominator and facilitate the analysis of $R \& D$ goals. In Section 6.0 we estimate allowable feedstock costs to establish attainability targets for mass culture production and to identify the best fuel products for production from microalgae. Then we determine the required improvements in fuel processing, first in reference to a single, subjective technology configuration that is judged to be attainable and then relative to uncertainties inherent in the attainability case. 


\section{Microalgae - The Simplest Plants}

Microalgae are the most primitive, and the most simply organized, members of the plant kingdom. The vast majority of microalgae exist as single cells in aqueous habitats, while a few are organized into simple colonies. Macroalgae-the seaweedshave achieved a further level of organization, with cells that are grouped into structures which resemble the leaves, stems, and roots of higher plants.

Almost twenty thousand species of algae have been identified. These species have been grouped into a number of divisions, three of which contain algae that are frequently employed for largescale cultivation. The members of the first group, the bluegreen algae, are organized similarly to bacteria in that their cells do not possess a nucleus. In some cases, the blue-green algae can utilize atmospheric nitrogen gas to fulfill their nitrogen requirements and thus serve an important ecological role as producers of fixed nitrogen. The remainder of the algae are true plants possessing nuclei and chloroplasts, which are the subcellular organelles in which photosynthesis takes place. The green algae (Chlorophyta) are among the most common, especially in fresh water, and can occur as single-celled or colonial forms. The golden algae (Chrysophyta) have developed more complex pigment systems and can appear yellow, brown, or orange in color. Golden algae can form oils (lipids) as their major energy storage product, in contrast to the majority of the green algae,

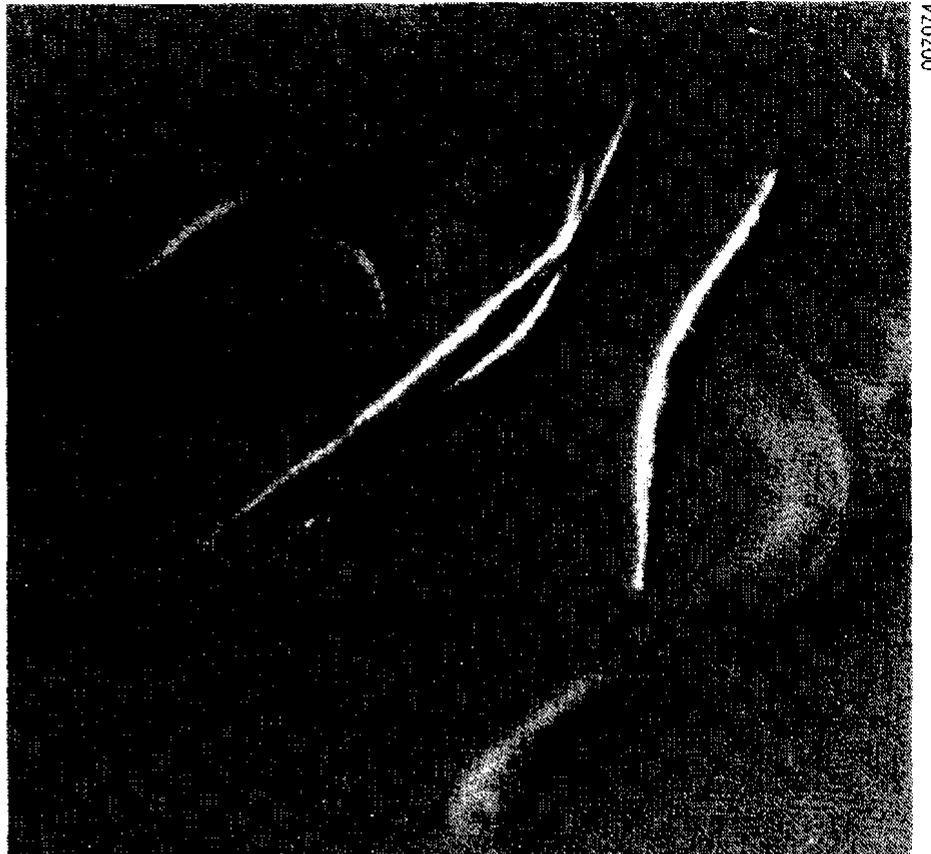

Ankistrodesmus faicatus - A single-cell green algae
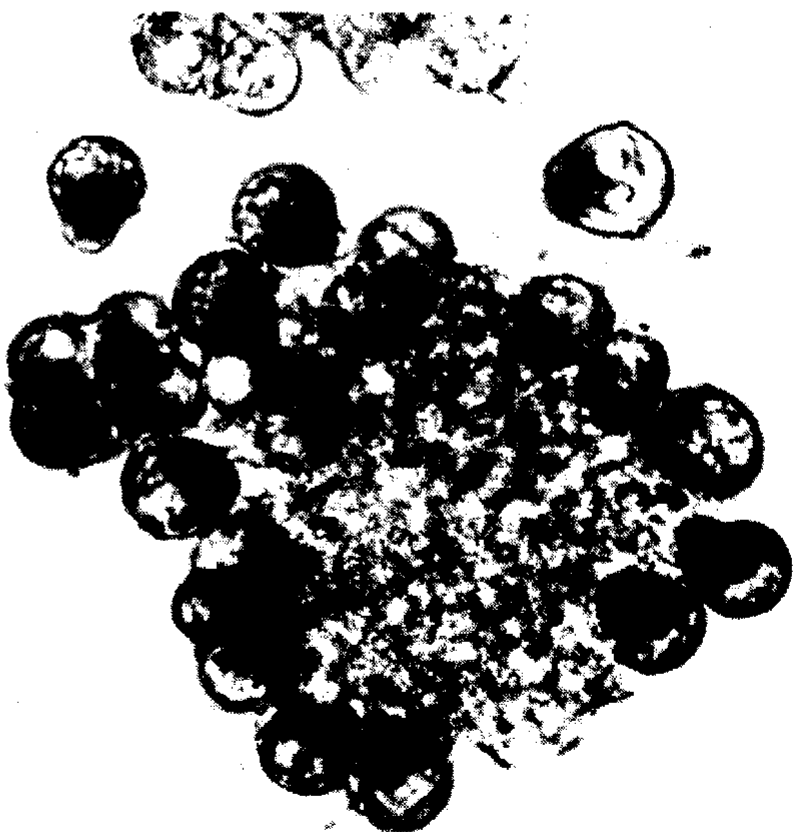

Colony of Botryococcus braunni 


\section{Microalgae - The Simplest Plants}

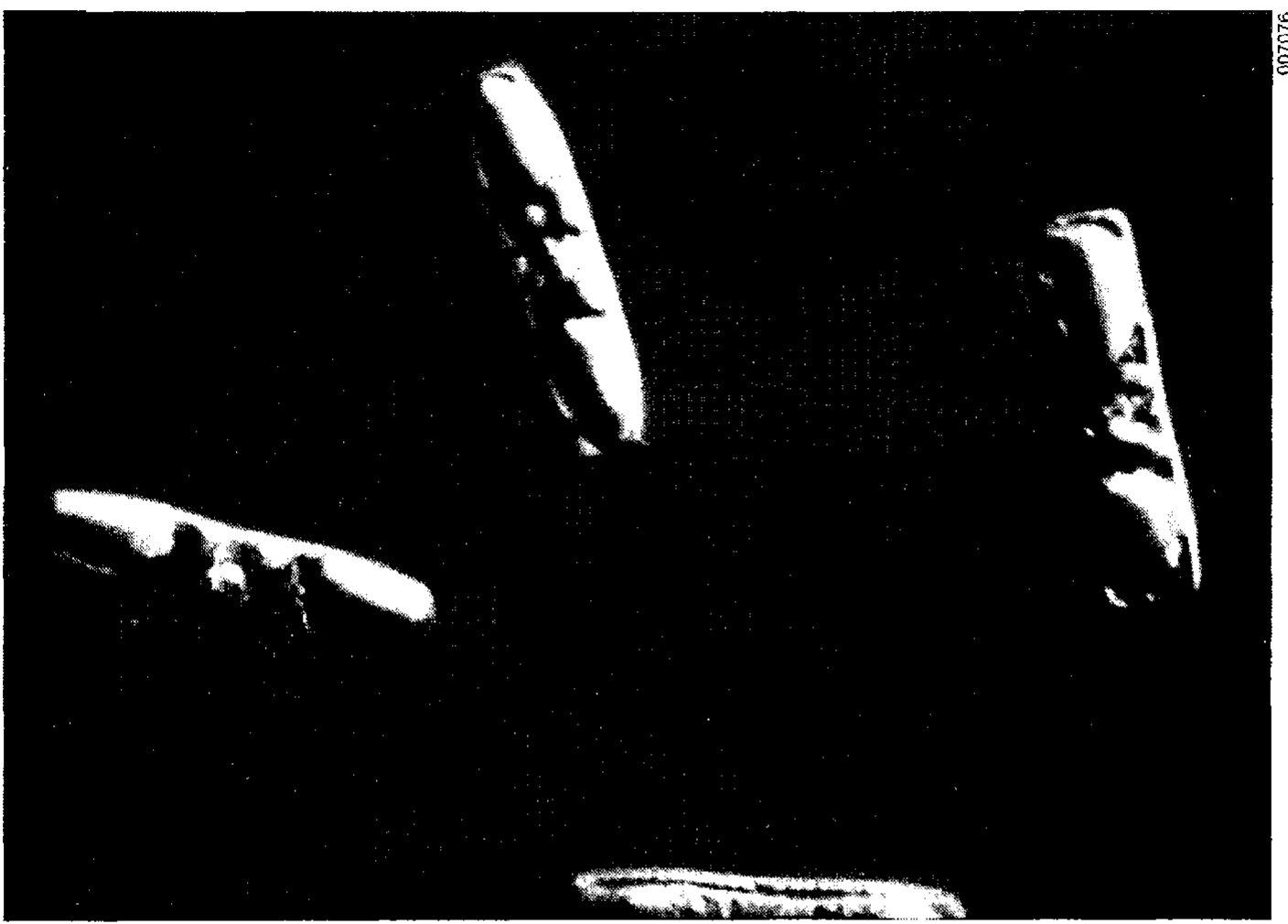

Hantzschia sp. A diatom (a golden algae) showing lipid accumulation

which produce starches. These oils can constitute $60 \%$ or more of the cell biomass under some conditions. Diatoms, a particularly common group of golden algae, normally form silica shells surrounding their cell mass.

Microalgae, as their name implies, are very small, typically about one one-thousandth of an inch in size. Their size varies widely, however; single-celled species larger than $1 / 4$ inch can be found, as can species which are as small as some of the bacteria, less than one tenthousandths of an inch.
While the biochernical mechanism of photosynthesis in algae is similar to that found in all plants, algae can be particularly efficient converters of solar energy to biomass by virtue of their "low overnead " Free of the need to generate elaborate support and reproductive structures, the microalgae can devote ine majority of the energy they trap to biomass growth. In addition, single cells suspended in an aqueous environment can directly acquire the water, carbon dioxide, and nutrients they require for growth. The energetic efficiency of the microalgae, along with their ability to produce large portions of their total biomass in the form of oils, has caused them to be regarded as one of the most promising crops for liquid fuels production from plant biomass. 


\subsection{PRINCIPLES OF MICROALGAL FUELS TECHNOLOGY}

This section is a primer on the principles of microalgae mass culture, engineering requirements and options, basic resource requirements, and fuel product alternatives. Sections 2.1 through 2.3 provide a review of basic concepts and establish the foundations for estimating production costs in Section 3.0. Section 2.4 describes the basic chemical components of algal biomass and develops the fundamental concepts for fuel process design and economics developed in Section 4.0.

\subsection{Mass Culture Technology}

\subsubsection{Introduction}

The fundamental components of a microalgal mass production technology (illustrated in the sidebar in Section 1.0) include water, nutrient, and carbon dioxide supply systems; culture ponds; harvesters; and processing equipment. In this section we describe the biological and engineering principles governing the design and performance of the components related to biomass production and harvesting. Processing components will be examined in Section 2.4.

\subsubsection{Culture Water Demand and Salt Balance}

Water demand and salt balance are governed by the evaporation rate, a function of climate and culture design, and by the salinity of the source water. Interactions among these variables are discussed first for uncovered and then for covered systems since this facility design variable affects the basic relationships. Then the effect of salinity on culture operation is discussed.

\section{Uncovered Systems}

One major advantage of microalgal production technologies is their ability to utilize saline waters, which are unsuitable for conventional agriculture or for higher plant production. Uncovered algal ponds lose significant amounts of water through evaporation, thus becoming solar concentrators of dissolved salts. Since many algal species can tolerate salinity well in excess of that of saline groundwater [4-25 g total dissolved solids (TDS)/L], and some species tolerate salinity in excess of that of seawater ( $35 \mathrm{~g}$ TDS/L), this concentration process is not viewed as detrimental within expected operating limits. However, constraints are placed on system operations by the upper limits of the algae's salt tolerance and by the solubility of various salts, and it is necessary to remove salts from the systems before concentrations reach these limits. Salt removal is achieved by a process termed "blowdown," in which a portion of the water in the culture is removed, carrying the appropriate amount of salt with it. Over the long term, the salt equilibrium in the system must be held within tolerable limits to maintain algal productivity.

The water volume consumed for blowdown is small relative to the source water input volume as long as the operating salinity of the culture is significantly greater than the source water and evaporation rates are not excessive. The remainder of the source water input volume replaces evaporation losses. Water for blowdown is ideally removed downstream of the harvester to minimize the loss of algal biomass with the blowdown stream: blowdown is achieved in the water recycle loop by replacement of part of the recycle with fresh water. 


\section{Covered Systems}

Transparent coverings over microalgal mass culture systems can reduce evaporative water losses, protect cultures from heavy rains and contamination, and permit improved temperature control. Such coverings, however, will add significantly to the cost of production systems. In addition to their cost, covers cause the following logistic problems:

- All covering materials will absorb and reflect portions (about $10 \%$ minimum) of the incident light, reducing the light utilization efficiency of the system. Water condensation on the inner surface of the covering and the accumulation of dust and dirt on the outer surface could reduce further the amount of light reaching the culture.

- While coverings can permit the maintenance of warmer cultures in the winter, they can cause severe overheating in the summer. This ef fect can be partially offset by the use of covering materials that reflect or absorb infrared radiation, but only at additional cost and with some loss of photosynthetically active radiation (PAR). Passive ventilation systems can be added to the covered systems to prevent overheating, but evaporation control, the main purpose of covers, would then be lost.

- Although culture systems are likely to be established in areas where there will be a net loss of water due to evaporation, severe rainstorms can be expected occasionally. If large areas are covered and protected from this rainfall, a significant problem of runoff and water disposal will be created unless mitigating actions are taken.

A comprehensive analysis of the trade-offs between the problems and the advantages of covered systems has not been performed. Engineering designs proposing covered or enclosed systems cannot be rejected out of hand, but their designers must thoroughly address these issues. In Section 6.0, we address minimum design requirements and tradeoffs between cost and productivity.

\section{Salinity Effects on Culture Performance}

Two aspects of the concentrations of salts in an algal culture medium influence growth: the total salinity or ionic strength of the solution, and the concentrations of the individual ions that make up the total salt content.

Microalgal species vary widely in their ability to tolerate salinity: while some species survive only in fresh water, other species can tolerate saturated brines (Bonin, Maestrini, and Leftly 1981). In uncovered cultures in arid regions, average operating salinities can be expected to be high due to high evaporation rates. However, since periodic rainstorms can be expected to lower the salinity temporarily, the most desirable species will be those that tolerate large fluctuations in salinity as well as high average salinity.

The concentrations of ions in solution have significant impacts on the growth of algal species. The major ions in saline waters (sodium, potassium, calcium, magnesium, chloride, and sulfate) are biologically active, but very few studies have been done on the effects of ionic concentrations on algal growth. Ions present in lower concentrations, which have been identified as limiting to algal growth in natural waters, have been studied intensively. These ions include nitrate, nitrite, ammonium, phosphate, carbonate, bicarbonate, and iron. Algal requirements for these low concentration nutrients and the ability of algae to assimilate them from the environment are well understood. Water added to algal cultures after evaporation or blowdown will carry nutrient ions that in 
some cases can help to meet nutrient demand. The significance of such contributions can be assessed only after more intensive study of specific saline water resources.

Salts in natural waters also include trace elements that, while vital to the survival and growth of microalgae, can become toxic to the algae in higher concentrations. These trace elements include copper, cobalt, zinc, manganese, molybdenum, and vanadium. Since algal cultures are expected to concentrate the groundwater salts, the effects of concentrating these ions must also be considered, especially with respect to the requirements of the algae and to the potential toxicity of these metals. The potential toxicity of other elements such as mercury, cadmium, and selenium must also be considered. Quantitative requirements of microalgae for trace elements have not been determined, and in most cases the levels at which the ions become toxic have not been well documented. It is known, however, that the presence of chelating agents in the water substantially reduces the activity of metal ions and hence their toxicity (Huntsman and Sunda 1980). It may be necessary to add chelating agents to culture systems, but some evidence shows that natural chelating substances produced by the algae may contribute to this detoxification function. The following aspects of water supply are of particular interest:

- Operating salinities. To minimize blowdown requirements, it may be desirable to operate systems at the highest possible salinities. High operating salinities can also reduce the possibility of contamination and may aid in predator control; however, the cells may require more energy to grow in a high salinity medium, which may be detrimental to productivity. Microalgal species that will perform well as fuel producers in high-salinity outdoor cultures must be identified and characterized. These microalgae must tolerate both the total ionic strength and the ionic composition of the culture source water.

- Requirements for nutrients. Most nutrients required by the algal cultures will be supplied through the addition of commercial fertilizers or other compounds, although some source waters can make significant contributions to these requirements (Section 2.1.4). Source waters can, in many cases, meet some of the requirements for major ions, including potassium, and can contribute to the requirements for nitrogen and phosphorus. If harvesting and processing mechanisms can be developed that permit significant nutrient recycling, the requirement for externally supplied nutrients can be further reduced. However, significant nutrient requirements will remain for startup or for facility expansion.

- Ionic toxicity. The concentrating effects of algal systems on potentially toxic trace elements and other toxic metals cannot be ignored. Since many of these elements are incorporated into the algal biomass, nutrient recycling systems will return these elements to the culture and further increase the concentration effects of the system. Thus, if the quantity of trace elements supplied by the source water exceeds the quantity removed in the product stream, elevated concentrations of these ions will develop. An understanding of the ion concentrations in the source water, the quantitative requirements of the algae, the toxicity responses of the algae, and the effects of natural and artificial chelation is necessary before this effect can be evaluated fully. 


\subsubsection{Carbon Dioxide Supply}

Algae utilize carbon dioxide $\left(\mathrm{CO}_{2}\right)$ as the source of carbon for the synthesis of organic compounds such as lipids. Since carbon demand increases in proportion to productivity, intensive mass culture of microalgae requires a large, reliable source of $\mathrm{CO}_{2}$.

The Necessity for Carbon Dioxide Supply

Carbon dioxide is present in the atmosphere at very low concentrations $(0.032 \%$ by volume in dry air). Passive flux of atmospheric $\mathrm{CO}_{2}$ through the surface of algal mass cultures is inadequate to supply the amount of carbon dioxide required to support intensive productivity. If a culture producing 25 grams of dry algal biomass per square meter per day was solely dependent on atmospheric $\mathrm{CO}_{2}$ as a carbon source, it would require all of the carbon dioxide in the air 50 meters directly above the surface of the culture each day. If this amount of air was bubbled through the cultures, the efficiency of transfer of $\mathrm{CO}_{2}$ from the air to the water would be extremely low, reflecting the low concentration of $\mathrm{CO}_{2}$ in the air. It would be necessary to bubble hundreds of cubic meters of air per square meter of culture each day to supply the necessary $\mathrm{CO}_{2}$. Moreover, it would be necessary to do this over the few hours of the day when the culture carbon demand was highest. The costs and logistics of such a system would be prohibitive. Thus, outdoor systems for the photosynthetic culture of microalgae have consistently required additions of $\mathrm{CO}_{2}$ to achieve high productivity.

\section{Carbon Dioxide Additions}

For the reasons outlined above, concentrated carbon dioxide must be added to systems to produce biomass fuels from microalgae. The sources of this carbon dioxide are discussed in Section 2.3.4. If $\mathrm{CO}_{2}$ additions to the culture raise concentrations above the equilibrium concentration with the atmosphere, significant quantities of $\mathrm{CO}_{2}$ will be lost (outgassed). Stringent automated control of $\mathrm{CO}_{2}$ additions will be required to minimize these losses, as will careful selection of the criteria governing $\mathrm{CO}_{2}$ addition so that a balance is achieved between possible $\mathrm{CO}_{2}$ limitation of the algae and $\mathrm{CO}_{2}$ loss to the atmosphere. The only major prospect for reduction of the total $\mathrm{CO}_{2}$ requirement is the utilization of the dissolved forms of carbon dioxide (primarily bicarbonate) that can be present in source waters. As with the nutrients discussed above, the large volumes of water added to the culture system to compensate for evaporation and blowdown may carry significant quantities of bicarbonate. Depending on the evaporation rate and the bicarbonate content of the source water, this water can supply from a few percent to more than $20 \%$ of the total carbon dioxide requirement.

\section{Carbon Dioxide Supply Systems}

Two factors determine the efficiency with which externally supplied carbon dioxide is converted into fixed organic carbon in microalgal systems. The first factor, carbon dioxide loss from the culture surface to the atmosphere, has been described. The second factor is the efficiency with which the carbon dioxide can be dissolved in the culture medium before it escapes to the atmosphere. If carbon dioxide is added as bubbles, large quantities can be lost if the bubbles reach the surface and burst. Alternatively, carbon dioxide may be added as small bubbles in counterflow columns, where the downward movement of the water offsets the upward movement of the bubbles until they are consumed. A third alternative supply mechanism holds the carbon dioxide in a containment vessel below the surface of the culture, and the free surface of the gas contacts the flowing water. Hybrid systems can also be envisioned. Since the addition of bicar- 
bonate salts to algal systems will lead to increased alkalinity, it seems unlikely that the bulk of $\mathrm{CO}_{2}$ will be added in this manner, and one of the above concepts must be developed to meet carbon requirements.

Little attention has been given to determining the efficiency with which carbon dioxide can be added to outdoor algal systems. Rough estimates from SERI subcontractors indicate that $50 \%-80 \%$ of the $\mathrm{CO}_{2}$ added to their systems is incorporated into the product algal biomass (Laws et al. 1983). Very little engineering effort has been devoted to the design of the $\mathrm{CO}_{2}$ addition systems used in these cultures, and significant improvements can be expected with improved engineering. If cultures are carefully monitored, if $\mathrm{CO}_{2}$ concentrations are maintained slightly below saturation with respect to the atmosphere, and if contained systems for $\mathrm{CO}_{2}$ addition are employed, highly efficient carbon dioxide utilization can probably be achieved without detrimental effects on algal production rates.

\subsubsection{Nutrient Supply}

Nutrient requirements are determined by basic biological requirements, the quality of the nutrients, and the design of the culture system.

\section{Algal Nutrient Requirements}

In the present context, nutrients can be defined as inorganic or organic compounds, other than carbon dioxide and water, that are incorporated into algal biomass during the process of growth, or whose presence in the cell is necessary to cellular function. Various compounds of over twenty elements are known to function as inorganic nutrients by this definition (Ukeles 1976). In addition, some algae require specific organic compounds synthesized by other organisms. However, many algae require only inorganic nutrients, and it is likely that these algae will be utilized for biomass fuel production.

Many inorganic nutrients required by algae are required in such low concentrations, or are present in natural waters in such relatively high concentrations, that they have not been observed to limit algal growth in nature (Huntsman and Sunda 1980). Nutrients that normally become limiting in outdoor mass cultures, as well as in natural populations, are nitrogen, phosphorus, silica (for diatoms), and iron. Algae can store nutrients in substantial excess of their requirements, presumably as a reserve against nutrient depletion; algal cells may contain 2-4 times the amount of nitrogen and more than 10 times the amount of phosphorus they require.

A major concern in algal mass culture is the regulation of nutrient consumption. The requirements for nutrients must be regarded as absolute, as these nutrients are components of the cellular "machinery" through which biomass is produced. Nevertheless, the consumption of these nutrients can be reduced if cellular processes can be directed away from the replication of cell machinery and toward the production of energy-rich storage products, which typically contain much smaller amounts of inorganic nutrients.

A two-stage system can be employed to induce algae to utilize their internal nutrient reserves for growth and product formation before harvest. Such a system may have important implications for the determination of product type as well (Section 2.1.5). Thus, the total nutrient content of the biomass product could be reduced, but it has yet to be determined whether such a reduction in algal nutrient content, and thus in the amount of nutrients required to produce a certain amount of biomass, can be achieved without detrimental effects on photosynthetic efficiency. However, if an efficient 
system of nutrient recycle can be developed, the nutrient content of the algal cells need not be a major concern, although the portion of the nutrients organically incorporated into the final product must always be replaced. In later sections we explore tradeoffs in production cost and recycle efficiency.

\section{Nutrient Sources}

The nutrients required by algae cultures can be supplied in a variety of forms. Many can be found in solution as a variety of ions or bound in organic compounds. Ionic forms can be supplied to the cultures as salts, both inorganic and organic. Nitrogen, for example, can be supplied as nitrate, nitrite, or ammonium ions combined with each other or with other ions in various salts; as urea; as ammonia gas or liquid; or as a wide variety of organic nitrogen compounds. The form in which nutrients are supplied is a major issue only for nutrients acquired in large quantities. The selection of nutrient source is most significant for nitrogen, because possible sources are numerous and the requirement is large.

Nitrogen source. Of the available nitrogen sources, ammonia $\left(\mathrm{NH}_{3}\right)$ and urea $\left[\mathrm{CO}\left(\mathrm{NH}_{2}\right)_{2}\right]$ are the least expensive. Ammonia can be supplied as a liquid under pressure, which reverts to the gaseous phase at atmospheric pressure. Nutrient handling costs are low with a pumpable fluid such as ammonia. In addition, ammonia is $83 \%$ nitrogen by weight. Urea is another attractive source of nitrogen. The cost of urea per nitrogen atom is similar to that of ammonia; however, handling can add to this cost, since urea is supplied as a crystal and cannot be inexpensively pumped. In addition, only $42 \%$ of the weight of urea is nitrogen; thus, more material by weight must be handled with urea than with ammonia. However, the urea molecule contains a carbon atom as well as two nitrogen atoms; this carbon atom is released as $\mathrm{CO}_{2}$ when urea is utilized, and presumably it is available for photosynthetic assimilation. Thus, urea can supply both the nitrogen requirement and $1.5 \%-10 \%$ of the carbon requirement. A more complete analysis of these trade-offs is required to determine the optimum nitrogen source.

Nutrient sources other than commercial fertilizers. Municipal wastewaters contain nutrients in forms that are readily available to algae or become available to the algae after bacterial decomposition. Bacterial-algal interactions for wastewater nutrient utilization have been studied extensively and are the basis of existing sewage treatment technology. Warm temperatures are a prerequisite for the operation of these systems, which are called high-rate oxidation ponds. Several obstacles restrict the use of sewage nutrients in biomass fuels production. Probably the greatest obstacles are the absence of sizable municipalities in the vicinity of anticipated production sites and alternate wastewater utilization technologies that are being developed and applied. In addition, municipal wastewaters do not provide a nutrient source concentrated enough to support intensive cultures of microalgae with a high population density in shallow water.

Since saline groundwaters contain significant quantities of certain nutrients, groundwater can contribute to the overall nutrient supply. However, the groundwater supplies nutrients in direct proportion to the volume of water utilized; thus, engineering strategies that minimize water use, such as covered systems, will also minimize the supply of nutrients from this source. Also, saline groundwaters vary widely in nutrient and nonnutrient ion content. There are insufficient data available to determine whether highnutrient (and in particular high-bicarbonate) groundwaters are available in sufficient quantities to support a microaigal fuels technology. Moreover, the long-term effects of pumping on the composition of these groundwaters is not known. 


\section{Nutrient Supply Strategies}

Careful regulation of the quantities of nutrients added to culture systems is extremely important. Two goals must be achieved: (1) minimization of the amount of nutrient lost with the water in the blowdown stream or to ammonia degassing, and (2) minimization of the nutrient content of the harvested algae, to the extent that this minimization does not significantly reduce product yield. Most mass culture systems operated to date have used nutrients in excess of the algae's requirement, constantly sustaining a high nutrient concentration in the culture medium. Thus, water removal from the system for blowdown and harvesting has led to significant nutrient loss and reduced efficiency.

Since the water expelled during blowdown is identical in composition to the culture medium, minimization of the nutrient loss to blowdown requires minimization of the standing concentrations of nutrients in the culture. Algae can grow rapidly at ambient nutrient concentrations that are nearly undetectable. In very dense outdoor cultures, nutrients are consumed quickly and nutrients must be added at the same rate at which they are consumed to maintain low concentrtions without nutrient limitation. This balance between addition and consumption can be achieved by continuous monitoring of nutrient concentrations in the culture combined with continuous additions designed to maintain low but satisfactory concentrations. However, reliable, easy-to-use instrumentation to monitor nutrient concentrations is not available.

\subsubsection{Algal Species for Mass Culture}

The design of microalgal mass culture systems is a synergistic process: the design must be tailored to the characteristics of the culture organism while species must be selected that contribute to economic construction and operation of the facility. Thus, a primary task in mass culture development is the "engineering" of the organism itself. In practice, these organisms are not amenable to direct engineering; rather, species with the most desirable characteristics must be selected from the thousands of natural species. Through breeding and molecular genetics, selected species can be altered to enhance these characteristics, and to suppress detrimental attributes.

\section{Characteristics of Suitable Species}

The first priority in selecting species for mass culture applications is to identify the desirable characteristics. This in turn requires some assumptions about the types of systems that will be employed and in particular about their potential location and the types of water that will be used. Many species characteristics considered desirable by the SERI microalgae program reflect the decision to locate the system in temperate latitudes in arid regions and to use saline source waters. These characteristics include (1) tolerance of high temperatures, (2) tolerance of a wide range of temperatures, (3) tolerance of high salinity, (4) tolerance of a wide range of salinity, and (5) high productivity at high incident-light intensity (resistance to photoinhibition). In addition, certain characteristics of species are desirable regardless of the location of the culture facility. These include (1) high photosynthetic efficiency, (2) size, buoyancy, or behavioral characteristics that enhance harvestability, (3) resistance to predators and contaminants, (4) absence of autotoxicity, (5) suspendability (absence of sticking or settling), (6) the ability to tolerate high nutrient concentrations, and (7) a life cycle that permits continuous culturing. Ideally, a species will also have a readily inducible sexual reproductive phase to permit genetic modification through classical breeding and selection. 
The final consideration, and one of the most important, is the slate of substances produced by the organism. In the Department of Energy's program, interest is focused on biomass products that can be converted to fuels. The characteristic of greatest interest is the fuel production rate, which is the biomass production rate multiplied by the fractional fuel product content. A species that exhibits a high biomass production rate under one set of conditions and a high fuel product content under another set of conditions cannot be considered desirable for fuel production systems, although it may be a good candidate for species development activities.

Species that fulfill all of the above criteria may not be available in the natural environment. The environment in a mass culture system differs significantly from most natural environments, and the performance criteria in mass culture (fuels production) are not equivalent to the performance criteria in nature (species survival and reproduction). Species must be collected from natural environments that reflect desired culture conditions as closely as possible, and these species must be screened for their fuels production ability early in the selection process. When species with the best possible combinations of the desired characteristics have been identified, development of these species through classical and molecular genetics can be undertaken.

\section{Species Development}

Since the natural environment does not select for algal species that will be optimum performers in mass cultures, it may be necessary to genetically alter these organisms through selective breeding (classical genetics) and genetic engineering (molecular genetics). In the classical genetic approach, cells that have desirable characteristics are mated, and their offspring are screened for attractive combinations of these characteristics. This process can be repeated many times, leading to significantly improved strains. Genetic engineering techniques permit the introduction of genetic material from other organisms or artificially synthesized genetic material into algae with good production potential, or direct modification of the algal genome. The following discussion presents basic issues relating to each of these approaches.

Selective breeding. In order for selective breeding to be successful, a genetic base must be developed. It is necessary to identify species that are suitable candidates for this process and to collect a large enough number of strains of each species to permit crossbreeding. The use of selective breeding is complicated because some algal species do not have known mechanisms of sexual reproduction, while others that have been observed to reproduce sexually cannot be induced to do so when required. Thus, improved knowledge of algal life cycles is required before sexual genetic recombination can be achieved.

An additional genetic technique is the creation of mutants through the use of radiation or chemical mutagens, and the subsequent selection of those mutants that offer improved characteristics for mass culture for fuels. The major drawback to mutagenic techniques is that the majority of mutations result in deletions of characteristics. Thus, mutagenesis is primarily applicable when characteristics are to be eliminated, for example regulatory mechanisms that prevent excessive accumulation of storage product. Mutagenic techniques can be utilized in combination with selective breeding techniques to suppress undesirable characteristics while adding or improving those that are desirable.

Genetic engineering. While genetic engineering permits direct manipulation of the physiological processes of the cell, many obstacles remain to the application of this technique to microalgae. To introduce genetic material into a host organism, it is necessary to have both a knowledge of the genetic material that governs the process to be 
altered and of a mechanism for introducing this material into the host cell (vector). Also, the physiology of the organism that receives an introduced gene will not always permit it to be expressed (to perform its physiological function). Genetic manipulation of the process of fuel (lipid) production in microalgae, for example, requires knowledge of the pathway by which lipids are produced, the mechanisms by which this pathway is regulated, and the discovery of a vector to introduce the necessary genetic alterations into viable cells of suitable species.

An additional technique that may hold some promise for species development is protoplast fusion. Some success has been achieved in generating protoplasts of macroalgae, but only limited success has been reported in regenerating viable plants from the protoplasts, or in obtaining fusion and regeneration (Cheney 1986; Saga, Bolne-Fuller, and Gibor 1986). Protoplasts are cells that lack a cell wall and are only surrounded by the plasma membrane. In some organisms, it has been possible to induce these protoplasts to merge and thus share their genetic material. This technique has been particularly successful in transferring characteristics genetically coded in the cytoplasmic organelles. Protoplast fusion techniques offer a form of genetic manipulation between classical breeding and genetic engineering. The application of this technique will require the development of techniques for protoplast formation and regeneration, and also the development of a genetic base of species and strain material.

\subsection{Design of Mass Culture Production Systems}

\subsubsection{Growth Conditions}

Growth conditions in microalgal mass cultures can be divided into two categories: those dictated by the location of the culture and those based on culture management strategy. Location-related variables include insolation, evaporation, rainfall, temperature, and wind velocity. Variables that can be managed include salinity, nutrient concentration, carbon dioxide concentration, culture mixing, culture aeration, and residence time of the population.

Growth conditions dictated by the culture's location will be among the prime considerations in siting the production facility. The SERI program has been based on the assumption that it will be necessary to locate a production facility in an area that receives large amounts of sunlight and has relatively warm temperatures.

Many other growth conditions in outdoor mass cultures are dictated by the management strategy selected. For example, through the blowdown technique mentioned earlier, the salinity of the culture can be maintained between the salinity of the source water and the algal salinity tolerance. The maintenance of selected nutrient concentrations and carbon dioxide concentrations has also been discussed. The rates of culture mixing, culture aeration, and culture turnover (detention time) can be selected. Culture management techniques also include the selection of strategies for the addition of medium, the supply of nutrients, the removal of product, and the selection of species for cultivation during various seasons. The major factors in the design and operation of mass culture systems are discussed below.

\section{Sunlight Requirements}

Since sunlight drives the production of biomass, ideal production systems will be located in areas that receive high insolation. Nevertheless, it is necessary to consider the tradeoffs involved in achieving high insolation. If the costs of land, raw materials, or 
operation are significantly increased at a location with high insolation, siting solely by the solar input may be disadvantageous. In addition, the search for areas of high insolation implicitly assumes that all solar radiation can be used by algal systems, regardless of its intensity. This assumption can be supported for well-mixed, energyintensive systems, but in less well mixed systems it is not clear that high-intensity light can be efficiently utilized.

\section{Culture Temperatures}

Algal growth rates have been positively correlated with culture temperature, at least until temperatures become high enough to be detrimental. However, algae in outdoor mass cultures do not normally grow near the maximum growth rate dictated by the temperature, but are limited in their growth by the availability of light or other factors. Properly selected species may continue growing with good photosynthetic efficiency even at low winter temperatures. However, low temperatures are normally correlated with low light intensities, and cultures at high or mid latitudes have become unproductive during the winter months (Benemann et al. 1977). While some limited level of production could be maintained at these times, productivity might be insufficient to support operational costs. While mechanisms to maintain higher culture temperatures during winter months might be suggested, these mechanisms will not be practical if light intensity falls below a critical level. Calibration of culture economics is needed to determine strong requirements.

\section{Culture Aeration}

The introduction of carbon dioxide into culture systems is necessary to achieve high rates of production, but other functions of aeration should not be overlooked. Among these is the reduction of culture oxygen concentrations during the highly productive midday period. During this period, culture concentrations can reach more than five times the equilibrium oxygen saturation concentration if no mechanism of vigorous gas exchange is provided. Oxygen, which is an end product of photosynthesis, inhibits the photosynthetic process when it is present at high concentrations through a process referred to as photorespiration.

The rate of photorespiration is determined by the relative concentrations of carbon dioxide and oxygen in the cell; when oxygen concentrations become too high, the photosynthetic process slows down--photorespiration becomes the limiting growth factor. Thus, to maintain productivity it is necessary either to provide a mechanism for oxygen removal from the culture during the highly productive midday period, or to select species that are particularly resistant to photorespiration. Culture aeration can remove excess oxygen--highly aerated systems have been shown not to become severely oversaturated-but may be excessively costly. Significant interspecific variability in resistance to photorespiration may exist among algae; it has been demonstrated that algae vary in their ability to concentrate $\mathrm{CO}_{2}$ within the cell (Miyachi, Tsuzuki, and Yagawa 1985). Since photorespiratory activity is determined by the relative concentrations of carbon dioxide and oxygen, this activity can be reduced by intracellular accumulation of $\mathrm{CO}_{2}$ as well as by the elimination of oxygen.

\section{Continuous and Batch Cultures}

There are three fundamental management strategies for cultures of suspended microorganisms: batch culture, continuous culture, and semicontinuous culture. In batch culture, a small inoculum of cells is added to a large volume of culture medium, and growth is allowed to continue until the desired population for harvest is obtained. In continuous 
culture, once the desired population is attained (usually through a batch startup procedure), fresh medium is continuously added and the biomass product is removed from the system at the same rate. Semicontinuous cultures are very similar to continuous cultures, except that portions of the population are removed and medium is added at discrete intervals, frequently on a daily basis.

Both continuous and semicontinuous cultures of fer significant advantages over batch cultures since population size and growth conditions can be maintained at or near a steadystate. If these conditions are near the optimum for growth and product formation, system productivity can be maximized. In batch culture, the population size is not optimal for most of the growth cycle. Recent culture management experiments (Laws 1984) have shown that semicontinuous strategies are particularly conducive to high production rates, although the physiological mechanism is not understood.

Species Utilization and Rotation

The operation of outdoor mass culture systems with harvesting and processing facilities can be simplified where a single algal species is maintained year round. However, the anticipated seasonal variations in culture conditions are wide enough that it is unlikely that a single species can be found which will perform optimally at all times. Thus, it seems more likely that mixed cultures will be employed, in which different species are dominant at different times.

The harvesting and processing characteristics of these different species must be similar enough that capital equipment can be used all year without significant modifications, particularly since multispecies populations may occur during transition periods. Maintaining startup populations of seasonally adapted species can also be a significant problem. It is desirable, but far from assured, that seasonal crops will remain at low levels in the culture at all times, and will reappear as conditions become suitable. Careful culture management would be required to avoid significant drops in system productivity during transitions between species or strains.

Product Manipulation

As noted in Section 2.1.4, the metabolism of microalgal cultures can be shifted from the production of cellular machinery toward the generation of storage products by nutrient deprivation. Lipid production can be induced by nitrogen deprivation in most algae, and by silicon deprivation in diatoms (Shifrin 1980). Through this mechanism, cells can be induced to accumulate a large proportion of their cell mass in the form of storage products. Chemical additions affecting cell biochemistry might also be utilized to induce storage product formation, although the economics of such mechanisms are questionable. It has not been determined whether the formation of storage products can be induced without significant reductions in photosynthetic efficiency. If a product induction phase is to be included in the culture system, it can be achieved by a number of mechanisms.

Most simply, cultures can be grown to a certain density with excess nutrients, and then nutrient additions discontinued until the desired degree of product accumulation is realized. For a single reactor, this implies batch culture operation. It may be more desirable, therefore, to achieve product induction through a system of linked continuous cultures. In such a system, cells would be produced in the first-stage continuous culture with ample nutrients available. The outflow from the first-stage culture would become the input to the second-stage continuous culture, which would receive little or no 
nutrient addition. Harvested material would be drawn from the second culture after a residence time that would be determined on the basis of maximum product formation rate. The advantages of continuous culture would apply for both stages of the linked system.

\subsubsection{Light Utilization in Mass Cultures}

Light utilization by algal mass cultures is determined by two factors: (1) the amount of the incident light that actually reaches the algal cells, and (2) the efficiency with which the light received is utilized. Losses of incident light that are not related to the photosynthetic efficiency of the algae themselves, but that influence the photosynthetic efficiency of the culture system as a whole, include losses of light to covering materials, reflection by the water surface, nonproductive areas of the facility such as roads and earthworks, and suspended particles other than algae in the culture medium. The loss of light as a result of the presence of covering materials has been discussed in Section 2.1.2. Loss of light to reflection by the water surface cannot be avoided and eliminates about $10 \%$ of the available radiation, although this loss is decreased somewhat by wind-induced ripples on the surface of the culture.

Once all light losses are accounted for, the utilization efficiency for the remainder of the light is determined by the photosynthetic efficiency of the algae themselves. The maximum efficiency with which light energy can be converted to the stored chemical energy of plant biomass is the theoretical efficiency of the photosynthetic process. When the energy contents of the quanta received from sunlight and the thermodynamic efficiencies of the photochemical conversion processes are considered, maximum theoretical photosynthetic efficiencies for sunlight utilization are estimated to be about $23 \%$ (Radmer and Kok 1979). Experimental observations that have shown efficiencies in excess of this maximum have been the subject of heated debate (Aiba and Ogawa 1983).

The practical limits of photosynthetic efficiency are below the theoretical efficiency. It is likely that the short-term utilization of light of low intensities can be very near this theoretical value, but plants typically utilize high-intensity light with much lower efficiency. Normally, increased light intensity beyond about $20 \%$ of full sunlight does not lead to a proportionate increase in the growth rates of cells. Thus, outdoor cultures with high light intensities have inherently low efficiencies. However, when outdoor populations are sufficiently dense, light is attenuated with depth in the cultures and only those cells at the surface receive light of very high intensity. In addition, algal cells can utilize high-intensity light at high efficiency if they are exposed to this light only for short periods of time. Thus, if cells in the culture are moved from the surface of the culture to the darker areas at depth, they can sustain a higher photosynthetic efficiency.

Nonproductive Areas of the Culture Facility

While the presence of nonproductive areas in the facility will reduce the overall light utilization efficiency, this effect is significant only to the extent that land costs contribute to the total production costs. Operating costs will be determined mainly on the basis of the actual area under cultivation. An exception is when barriers or earthworks shade parts of the cultures. This suggests that barriers between culture channels should be located as far apart as the hydraulics of the system will permit, and that their height should be minimized. 


\section{Control of Nonalgal Turbidity in Cultures}

While care must be exercised to prevent the accumulation of dust, dirt, and chemical precipitates in the culture medium, with reasonable care it seems likely that the only major potential source of nonalgal turbidity will be bacterial biomass. In cultures supplied with inorganic fertilizers, bacterial populations are normally small when culture growth remains vigorous. Where municipal wastewater or some forms of nutrient recycling are employed, bacterial stocks may be much higher, due to the presence of organic substrates.

\section{Culture Mixing}

It has been demonstrated that the application of vertical mixing to outdoor cultures can significantly enhance their photosynthetic efficiency and hence their productivity [e.g., Laws et al. (1983)]. It is less clear, however, whether this sort of enhancement can be cost-effective, since mixing can be extremely costly. This trade-off can only be quantified through the resolution of several key issues, including the amount of mixing required to achieve a given degree of enhancement and the energy consumption of the hydraulic systems employed to generate the required mixing. However, mixing designed to enhance photosynthetic efficiency need only be applied during the few hours of the day when the incident light intensity is high. Mixing effects other than the direct enhancement of photosynthetic efficiency must also be considered in analyzing this trade-off; these effects include enhanced nutrient transfer across the cell boundary layer and the maintenance of a suspended cell population.

\subsubsection{Engineering and Construction}

Although many pond designs have been employed during the forty-year history of microalgal mass culture, a single design is now used almost exclusively. This design employs a shallow pond, $15-50 \mathrm{~cm}$ deep, configured in a racetrack or meandering style, through which water is circulated at a moderate rate to retain culture suspension. A motorized paddlewheel is commonly employed to circulate the water. The materials utilized for the construction of such ponds vary widely.

Paddlewheels offer advantages over other pumping systems in the energetic efficiency of water movement and in the avoidance of mechanical damage to the algal populations. Other circulation systems have been tested as well. Air-driven systems have been a particularly popular alternative, whether in the form of airlift pumps or direct culture bubbling, but their energetic efficiency is much lower than that of paddlewheel systems.

There are limits to the temperature tolerance of algal species, and in many cases the fluctuation of temperatures in outdoor cultures of microalgae exceeds these limits, causing culture failure or contamination. The identification of temperature-tolerant algae has been a major objective of the SERI program, but absolute temperature control in outdoor cultures may be possible.

\section{Pond Configuration}

The pond design described above has been widely accepted for several reasons. Construction is inexpensive relative to most of the other designs proposed, and culture circulation is achieved efficiently. However, this design has not been the most productive 
of all designs tested (Terry and Raymond 1985); its selection is based on productivity/ cost trade-offs, rather than on the optimization of production. While the merits of the presently accepted design should be recognized, it should not be considered to be either optimum or final; innovation should be encouraged.

\section{$\underline{\text { Pond Materials }}$}

Ponds have been constructed with a wide variety of bottom materials, including compacted soils, a thin blanket of crushed rock or other erosion-resistant material over impervious soil, soil cement, concrete, and plastic liners. These materials vary in roughness, stability, permeability, and resistance to failure. Surface roughness determines both the resistance to water movement and the potential for cell aggregation. Unstable bottom materials are subject to shifting and settling, which can destroy the original hydraulic design of the facility. Permeability or leakage (particularly in the case of plastics) can lead to the loss of both water and algae. Failures, including cracking of the bottom and the breakdown of the culture sides, lead to high maintenance cost and lost production. The construction of very large culture facilities for products such as fuels, in view of the potential scale of the technology, could support the development of specialized techniques for lining once design requirements and cost/performance tradeoffs have been identified.

\section{Culture Circulation}

The paddlewheel circulation system described above is regarded as the best design currently available. Again, it cannot be accepted as the optimal design, and research in alternative circulation systems is ongoing (Terry and Raymond 1985).

\section{Artificial Temperature Control}

Culture heating and cooling by conventional means can almost certainly be eliminated for energy production systems, since the necessary energy input would outweigh the energy production of the system and lead to an unacceptable negative energy balance. Alternative techniques, including passive solar and geothermal heating deserve consideration, although they pose significant logistic barriers. Alternative techniques for culture cooling will be particularly difficult to develop, and the selection of species that tolerate high temperatures appears to be a desirable objective since it offers a means of mitigating the deleterious effects of temperature.

\subsubsection{Harvesting}

\section{General Harvesting Considerations}

Techniques for harvesting microalgae include settling or flotation, centrifugation, and filtration. These processes are aided by cell flocculation, either through the addition of chemical flocculants or through culture autoflocculation. Flocculation causes the cells to become aggregated into larger clumps; these clumps are more easily filtered and settle more rapidly, either through gravity settling or through accelerated settling by centrifugation. Flotation is achieved through the introduction of small bubbles into the culture medium; algae are attracted to these bubbles and lifted to the surface for removal.

Alternative harvesting techniques. The present slate of harvesting techniques for microalgae is costly both in terms of capital investment and in terms of energy input. It is not clear whether these techniques can be significantly improved, but the total engineering 
investment in developing these techniques to date is small relative to the potential scale of the technology. Continued innovation and research in the area of microalgal harvesting may offer significant improvements in harvesting costs.

Salinity effects on harvesting procedures. Many of the procedures developed for the harvesting of algae from freshwater systems or from wastewater systems have been based on the interaction between cells and various charged particles employed as flocculants. Since the effectiveness of these flocculants is significantly reduced in waters of high ionic strength, greatly increased doses of flocculant are required to achieve the desired effect at high salinities.

Chemical flocculation. Chemical flocculation substantially increases cell harvestability, particularly for small unicellular species. However, the addition of flocculants introduces chemicals into the product stream and into the culture itself if the medium is to be recycled after harvesting. Autoflocculation processes can take advantage of $\mathrm{pH}$ increases caused by the growth of the algae to stimulate flocculation, but these processes probably depend on the formation of precipitates such as calcium phosphate in the medium, and these ions must subsequently be replaced. In addition, autoflocculation processes are less effective at high salinities.

\section{Biological Aspects of Harvesting}

The harvestability of microalgae depends primarily on the organism's size, which determines the settleability and filterability of the species. Cell motility may also have a significant effect on harvesting processes that depend on settling. Cell surface charge, aggregation properties of the cells in culture, and behavioral characteristics of the cells may enhance or decrease their harvestability.

Species harvestability. The most rapidly growing algal species are frequently very small, and often motile, unicells, which are among the most difficult to harvest. It is necessary to maintain an effective interaction between the development of harvesting technologies and the isolation and selection of algal species for possible mass culture applications to ensure that the species selected can be harvested by techniques presently in practice or under development. While harvestability cannot be an absolute requirement for species selection, species readily harvestable by known techniques should receive preferential attention in species development activities.

Environmental manipulation of harvestability. Many of the harvesting processes currently in use depend on the electrical charge at the surface of microalgal cells. There is some evidence that the strength of this charge varies with the growth conditions of the algae (Sherbet 1978). If so, the growth conditions might be manipulated to improve cell harvestability. Characteristics such as cell size and colony formation can also be manipulated through variation of growth conditions and can affect harvestability. Where growth conditions are manipulated to improve harvestability, however, the effects of these manipulations on production must be taken into account.

Biological activity in harvested material. For harvesting techniques such as settling, which takes place relatively slowly and may require a long residence time, consideration must be given to the biological activity within the concentrated material. Metabolic activity of the cells can lead to the development of anaerobic conditions, cell death, and decomposition by autolytic and bacterial processes. Storage of algal cells in the dark will lead to storage product utilization and loss of the fuel product. Where timedependent harvesting processes such as long-term settling are to be utilized, such potential losses must be considered. 


\subsubsection{Summary}

The wide variety of unresolved issues in mass culture technology is strongly indicative of the state of the art. As an emerging biotechnology, microalgal mass culture remains in its infancy. Although present microalgal technologies, based on high-value products, can survive without the complete resolution of these issues, systems for the production of high-volume, low-value products such as fuels will require resolution of key issues.

These issues form the basis for the identification of research needs. The broad range of questions that must be answered, however, indicates that priorities for research activities must be established, and that the questions most crucial to the development of process viability must receive priority. The identification of the most crucial questions, however, is not always straightforward. Our economic analysis that begins in Section 3.0 examines the response of product cost to various systems parameters; the relative sensitivity of product cost to these parameters can help to suggest priorities for research.

\subsection{Resource Requirements}

As part of the development of the mass microalgal culture concept, SERI has conducted and sponsored studies to determine the process requirements for major resources. A study by Vigon et al. (1982) described the resources of the desert in the southwestern United States and factors affecting their availability for production of microalgal or emergent aquatic plant species. Resource availabilities are outlined in this study along with recommendations for measuring the quantity of each resource available at a particular site.

A subsequent resource evaluation by Maxwell et al. (1984) suggested screening procedures for resource evaluation. Land, water, and climatic criteria were established, quantitative and qualitative data were assembled, and through the use of quantitative weights, multivariate criteria were combined into a single measure of "suitability" for the Southwest.

These studies discuss relevant resource requirements and related issues that are too extensive to reiterate here. Instead, we summarize the major resource issues that relate to land, climate, and water. For carbon dioxide, we provide a more extensive synthesis of several evaluations of supply options and competing demands for this critical resource.

\subsubsection{Land Requirements}

The mass culture of microalgae can be envisioned as a series of ponds connected by hydraulic and utility grids. Several physical characteristics of the land affect the construction and maintenance of the ponds. We group these characteristics into two general categories, physical requirements and location requirements, and briefly review concepts that are discussed in detail elsewhere (Benemann et al. 1982; Vigon et al. 1982; Maxwell et al. 1984).

\section{Physical Requirements}

To maintain culture hydraulics in a flowing culture $10-20 \mathrm{~cm}$ deep, ponds must be graded to acceptable tolerances and maintained within these tolerances. Land of uneven topography or severe slope presents difficulties in the grading and preparation of ponds; 
the flatter the land, the less grading is required to achieve the basic pond gradients. Soil type also affects site preparation costs through grading requirements and pond design. Soils of low porosity and permeability may provide sufficient barriers to water loss to preclude the use of liners (Dodd 1984).

\section{Location Requirements}

The physical requirements of land for sustaining algal culture place no strict contraints on their location. Factors such as ownership can exclude certain tracts under existing social or political arrangements, but these factors relate to prevailing political, social, and economic conditions and do not reflect the requirements of the algae. Climatic conditions, which are joint properties of land resources, are important factors in algal culturing and are discussed next.

\subsubsection{Climate Requirements}

Since microalgae rely on sunlight to energize their metabolism, the availability of sunlight is a major factor in determining the production rate. Average daily, monthly, and annual insolation determines the total energy input to the culture. Factors such as cloud cover and atmospheric turbidity also limit the amount of sunlight that reaches the culture and reduce the net energy available for production (Maxwell et al. 1984).

Other climatic conditions do not necessarily preclude mass culture of microalgae but may limit production. Rain or snow dilutes open cultures and may temporarily disrupt culture salinity balance and reduce productivity. Storms can deposit dust or debris in the cuiture and inhibit algal growth. Violent climatic events can damage the facility or its components, but these risks are undertaken in any agricultural enterprise and are not absolute barriers to feasibility.

\subsubsection{Water Requirements}

A major factor in determining water needs is evaporation, the rate at which water is lost to the atmosphere. Evaporation rates are characteristic of the environment and, unless reduced by some protective device, are fixed and calculable by site.

Water demand is further influenced by the source water characteristics and the algae themselves. Algae are endowed with a specific salinity tolerance. A closed system can maintain salinity at or near the salinity of the source water. Open systems suffer losses through evaporation; thus salinity rises and eventually the tolerance limits can be exceeded. The blowdown process expels water from the culture, replacing it with the source water and lowering salinity to within tolerance. However, water demand is increased through blowdown, which is required more of ten for more saline source water and for greater evaporation rates. Harvesting can also increase water demand in an open system lacking complete recycling. Precipitation could reduce water demand but is beneficial only if it does not endanger culture maintenance.

Water demands for mass culture of microalgae depend on complex, interrelated issues and factors, some dictated by natural conditions and some determined by management strategies and therefore by system economics and objectives. At a minimum exact calculations of culture water demands require sufficient site specificity to determine evaporation rates and source water salinity. With these dimensions fixed, trade-offs in water costs becomes important determinants in optimizing depth, blowdown, and harvesting strategies. 


\subsubsection{Carbon Dioxide Supply and Demand}

This section discusses the potential supply and demand of carbon dioxide in the southwestern United States. The importance of carbon dioxide as an inorganic nutrient for mass cultivation of microalgae is described in Section 2.1.3. Previous studies have suggested that the lack of adequate supplies of carbon dioxide might be a severe constraint to optimal production (Benemann et al. 1982; Nelson 1982; Vigon et al. 1982).

A multimillion dollar industry supplies carbon dioxide for industrial uses such as chemical manufacturing (e.g., urea and methanol), refrigeration, and beverage carbonation. During the 1970s industrial demand for carbon dioxide increased at an average annual rate of $7.3 \%$. In addition, use of $\mathrm{CO}_{2}$ in enhanced oil recovery (EOR) projects has created a new, potentially large demand that could significantly impact the price and supply of $\mathrm{CO}_{2}$ (Meegan 1977).

National Demand for Carbon Dioxide for Enhanced Oil Recovery

The demand for carbon dioxide in enhanced oil recovery (EOR) projects will depend on the future price for crude petroleum and government tax policy. At lower crude petroleum prices, the value of carbon dioxide flooding declines dramatically because EOR projects require heavy front-end investments and the purchase of carbon dioxide represents about $47 \%$ of the production cost. Carbon dioxide-based EOR projects currently account for over $6.5 \%$ of all EOR-produced oil in the United States.

In a recent study on enhanced oil recovery, the National Petroleum Council (1984) estimated that an additional 14.5 billion barrels (bbl) of oil could be produced nationally using current EOR technology with stable $(\$ 30-\$ 35 / \mathrm{bbl})$ petroleum crude prices. Carbon dioxide miscible flooding is anticipated to contribute over $38 \%$ (or 5.5 billion bbl) to this incremental increase in recoverable oil reserves. Using $\mathrm{NPC}$ projections, $\mathrm{CO}_{2}$-based oil production is expected to increase from the current $31,300 \mathrm{bbl} /$ day to a peak of $500,000 \mathrm{bbl} /$ day by the year 2000 . After 2010 , this production rate is predicted to decline due to reservoir depletion.

Total carbon dioxide demand for the EOR projęcts in the United States has been estimated to range from 850 billion to $\frac{1.7}{3.7}$ trillion $\mathrm{m}^{3}$. This estimate is based on a $\mathrm{CO}_{2}$ utilization rate between 140 and $280 \mathrm{~m}^{3}$ per barrel of recovered oil. Daily demand estimates for carbon dioxide for EOR projects depend on assumptions regarding extended lifetimes for oil fields using $\mathrm{CO}_{2}$ recovery. Lifetimes for carbon dioxide fields range from 10 to 20 years, depending on the characteristics of the reservoir and price of $\mathrm{CO}_{2}$. Through the lifetime of an EOR project, the yield of oil diminishes to a point where it is no longer economical to inject $\mathrm{CO}_{2}$ for additional recovery. However, over the next forty years, the national average daily demand for $\mathrm{CO}_{2}$ is estimated to be over 100 million $\mathrm{m}^{3} /$ day, assuming net $\mathrm{CO}_{2}$ requirements of $280 \mathrm{~m} / \mathrm{bbl}$. The peak daily demand for $\mathrm{CO}_{2}$ is estimated to be 140 million $\mathrm{m}^{3}$ for an oil production rate of $500,000 \mathrm{bbl} / \mathrm{day}$.

Demand for $\mathrm{CO}_{2}$ in the Southwestern United States

The demand for carbon dioxide for EOR is not uniformly distributed throughout the United States. In fact, the NPC study (1984) indicates that over $56 \%$ of the oil reservoirs most amenable to $\mathrm{CO}_{2}$ recovery are located in the Permian Basin of West Texas and eastern New Mexico. The Permian Basin is estimated to contain 3.1 billion bbl of oil recoverable using $\mathrm{CO}_{2}$. Of the $63 \mathrm{CO}_{2}$ projects under production or construction, 35 are 


\section{Saline Groundwater}

In its usual sense, groundwater is defined as subsurface water below the water table in fully saturated geologic and soil formations. A broader concept includes very deep, saturated geologic formations and nearsurface, unsaturated soil moisture regimes. Inflow to the hydrologic system occurs as precipitation in the form of rainfall and snowmelt. Water may then move by surface flow, subsurface flow, and evapotranspiration from land to the ocean or atmosphere.

Water arriving at the soil surface as rain or snowmelt can be described chemically as an extremely dilute, slightly to moderately acidic, oxidizing solution containing low quantities of dissolved solids [several to tens of milligrams per liter $(\mathrm{mg} / \mathrm{L})]$. As it infiltrates the soil zone, water usually becomes considerably more acidic due to soluble $\mathrm{CO}_{2}-$ from root respiration and oxidation of organic matter- with which it reacts to form carbonic acid $\left(\mathrm{H}_{2} \mathrm{CO}_{3}\right)$. The $\mathrm{H}_{2} \mathrm{CO}_{3}$-enriched water encounters minerals that are dissolvable in numerous sequences of reactions within a system that is more or less continuously recharged with additional inputs. As groundwater moves along its flow path, the content of dissolved solids and major ions increases, ultimately becoming similar in composition to seawater. With increasing depth below the surface, residence time, and sluggishness of flow, the dominant anions tend to evolve from bicarbonate $\left(\mathrm{HCO}_{3}{ }^{-}\right)$to sulfate $\left(\mathrm{SO}_{4}{ }^{2-}\right)$ to chloride $\left(\mathrm{Cl}^{-}\right)$. Solubility sequences among the major cations-potassium $\left(\mathrm{K}^{-}\right)$, sodium $\left(\mathrm{Na}^{+}\right)$, calcium $\left(\mathrm{Ca}^{2}\right)$, and magnesium $\left(\mathrm{Mg}^{2 \cdot}\right)$ -are more variable due to cation exchange reactions. The species and concentration of both cations and anions are affected by the geochemical characteristics of the lithology

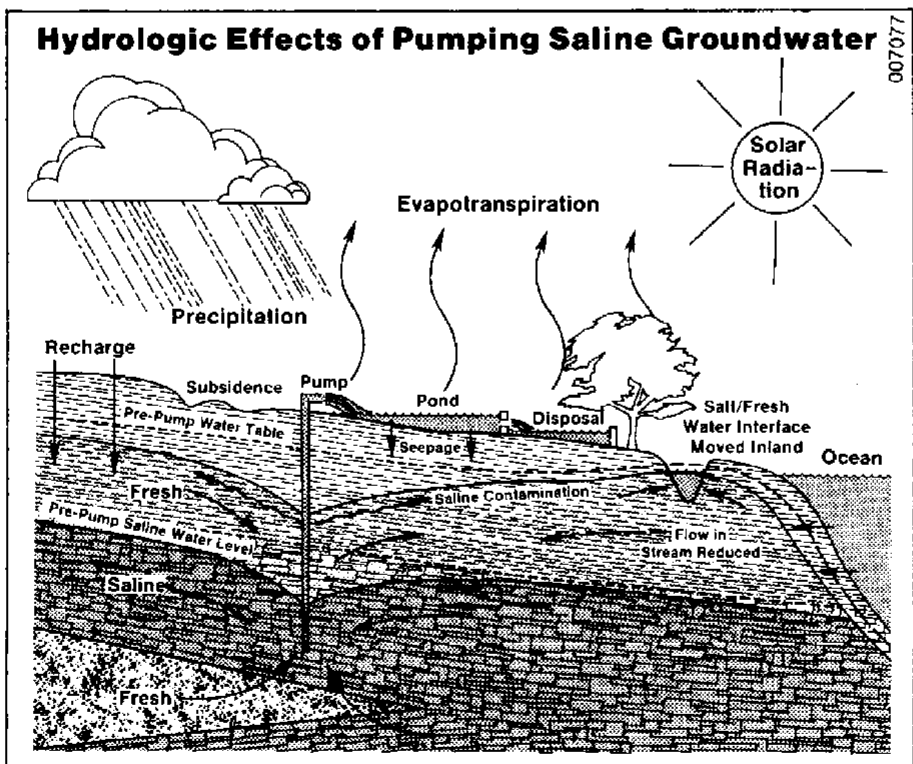

\begin{tabular}{cc}
\hline $\begin{array}{c}\text { A Groundwater Classification } \\
\text { Based on Total Dissolved Solids }\end{array}$ \\
\hline Description & g TDS/L \\
\hline Fresh & $0-1$ \\
Brackish & $1-10$ \\
Saline & $10-100$ \\
Brine & $>100$
\end{tabular}

through which the groundwater moves. Chloride content, for example, may be substantially increased if the flow encounters strata of evaporated marine basins.

The tendency of groundwater to become mineralized is most pronounced in arid regions such as the U.S. Southwest, where the potential evaporation exceeds the precipitation. Near-surface strata and soils frequently become saline or alkaline due to deposition of dissolved minerals during evaporation of soil moisture. including that carried upward by capillary flow. In confined topographic basins, which are common in the Southwest, the groundwater may gain dissolved minerals as water movement alternates between downward infiltration and upward migration in response to discontinuous recharge and the evaporative pull. At greater depths, the mineralization sequence may be enhanced by the occurrence of salts in ancient marine deposits.

Groundwater can be classified according to the amount of dissolved inorganic chemical constituents, or concentration of total dissolved solids (TDS), it contains. One commonly used scheme is presented in the table above. Water containing up to 2 to 3 gTDS/L may be tolerated for human consumption provided toxic 


\section{Saline Groundwater}

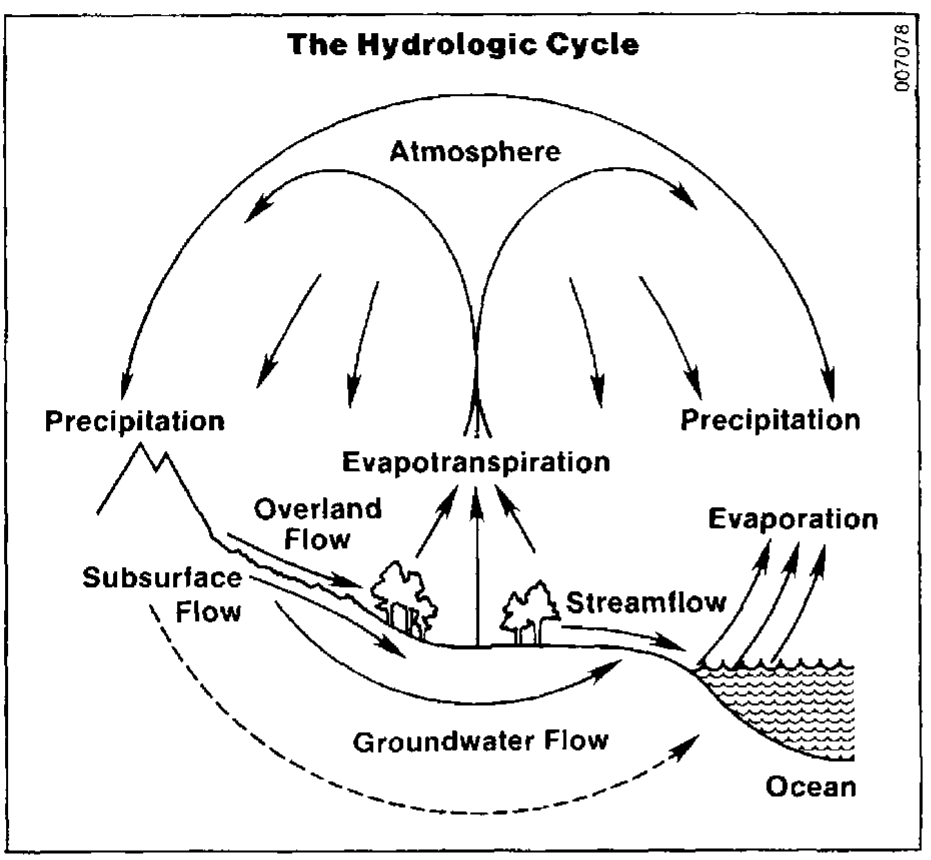

constituents such as selenium lead, and arsenic are virtually absent. Livestock may tolerate from 4 to $10 \mathrm{~g}$ TDS/L. Most commercially useful plants have lower tolerances than humans and livestock.

Seawater has a TDS concentration of about $35 \mathrm{~g} / \mathrm{L}$. The TDS concentrations of confined basins such as Great Salt Lake substantially exceed that of seawater.

The occurrence and characteristics of saline groundwater in the U.S Southwest are poorly known due to lack of interest in it as a resource. The principal source of available information is the Geological Survey of the U.S. Department of Interior. Existing data can be viewed as data of opportunity - that is, they have resulted largely from other purposes, such as exploration for petroleum or fresh water. rather than from deliberate saline groundwater investigations.

If three major phases of groundwater development are identified as exploration, evaluation, and exploitation. the status of our understanding of saline ground water in the U.S. Southwest lies somewhere between exploration and evaluation. The occurrence of major aquifer systems is probably known, but their characteristics of recharge, discharge, and confinement are not well understood. Even more sparse is information on hydrologic features such as lithology, chemical quality, prospective yields, and potential undesirable side effects. Information developed with models and field data collection, such as electric logs and pumping tests, are needed to determine the location, number, spacing, yield, and effects of wells. Development of saline groundwater for microalgae culture should, in particular, consider effects such as land subsidence, fresh water contamination, and drawdown of the water table.

\section{References}

1. Davis, S.N., 1974, Hydrogeology of Arid Regions, in Brown, Jr., G.W. (ed.) Desert Biology, Volume II, Academic Press, New York.

2. Feth, J.H. et al., 1965 Preliminary Map of the Conterminous United States Showing Depth to and Quality of Shallowest Ground-Water Containing more than 1,000 Parts Per Million Dissolved Solids, Hydrologic Investigations Atlas HA-199, Geological Survey, U.S. Department of the Interior.

3. Freeze, R.A. and J.A. Cherry 1979, Groundwater, PrenticeHall, Inc., Englewood Cliffs, NJ.

\section{Summary Appraisals of the} Nation's Ground-Water

Resources, Professional Paper 813 series, Geological Survey, U.S. Department of the interior.

5. Maxwell et al., 1984,

Resource Evaluation and Site Selection for Microalgae

Production Systems, SERI/TR215-2484, NTIS, Springfield, VA 
in the Permian Basin. Total carbon dioxide demand in this basin could reach 880 billion $\mathrm{m}^{3}$ ( 120 million $\mathrm{m}^{3} /$ day) over the next twenty years. This estimate for the Permian Basin is based on a shorter time frame than the national estimate because most oil fields within the Permian Basin are expected to be developed first and therefore depleted first.

Comparable estimates for carbon dioxide demand were developed in a study conducted jointly by Science Applications, Inc. (SAI) and several large petroleum production companies. The demand data developed in this report, presented in Table 2-1, indicate a similar, albeit higher, total demand estimate for $\mathrm{CO}_{2}$ in the Permian Basin. This inconsistency in demand forecast is due to different assumptions in net $\mathrm{CO}_{2}$ utilization, reservoir lifetime, and crude oil prices between study periods. The geographic distribution in Table 2-1 presents a higher percentage of $\mathrm{CO}_{2}$ utilization in the Permian Basin, where total demand for $\mathrm{CO}_{2}$ is 1.2 trillion $\mathrm{m}^{3}$ or 230 million $\mathrm{m}^{3} /$ day. If reservoir lifetime were assumed to be twenty years, the adjusted $\mathrm{CO}_{2}$ demand from the SAI study is about 180 million $\mathrm{m}^{3} /$ day. This daily dęmand estimate is more consistent with $\mathrm{NPC} \mathrm{CO}_{2}$ demand projections (120 million $\mathrm{m}^{3} /$ day) given anticipated real oil price increases and new EOR technologies in the future.

In summary, the demand for $\mathrm{CO}_{2}$ for enhanced oil recovery in the Southwest could vary from 120 million to 180 million $\mathrm{m}^{3} /$ day over the next twenty years if crude oil prices remain relatively stable (in real terms) between $\$ 30$ and $\$ 40 / \mathrm{bbl}$. Lower crude prices would reduce $\mathrm{CO}_{2}$ demand because EOR would be less profitable. Higher prices might accelerate $\mathrm{EOR}$ profits and $\mathrm{CO}_{2}$ demand, or stimulate drilling and result in the opposite effect as a result of tax benefits for new sources of oil.

Carbon Dioxide Supply - National Estimates

Carbon dioxide is obtained from three major sources:

- Naturally occurring $\mathrm{CO}_{2}$ deposits or $\mathrm{CO}_{2}$ located in conjunction with natural gas deposits;

Table 2-1. Potential $\mathrm{CO}_{2}$ Demand by Petroleum Basin

\begin{tabular}{lccc}
\hline Basin & $\begin{array}{c}\text { Total } \\
\text { Demand } \\
\left(10^{12} \mathrm{~m}^{3}\right)\end{array}$ & $\begin{array}{c}\text { Daily } \\
\text { Demand over } \\
\text { a 15-yr } \\
\text { Period } \\
\left(10^{6} \mathrm{~m}^{3}\right)\end{array}$ & $\begin{array}{c}\text { Daily } \\
\text { Demand over } \\
\text { a 20-yr } \\
\text { Period } \\
\left(10^{6} \mathrm{~m}^{3}\right)\end{array}$ \\
\hline Permian & 1.28 & 233.1 & 174.82 \\
Williston & 0.03 & 5.51 & 4.11 \\
Appalachian & 0.01 & 1.93 & 1.42 \\
Los Angeles & 0.05 & 8.75 & 6.54 \\
Total & 1.37 & 249.30 & 186.88 \\
\hline To convert cubic meters $\left(\mathrm{m}^{3}\right)$ to cubic feet, divide $\mathrm{m}^{3}$ by 35.31. \\
Source: Anada et al. 1983.
\end{tabular}


- By-product $\mathrm{CO}_{2}$ from the manufacture of chemicals, fertilizers, alcohol, and coal gasification plants; and

- $\mathrm{CO}_{2}$ recovered from the flue gas of fossil fuel combustion plants.

National reserves and by-product sources of $\mathrm{CO}_{2}$, developed by SAI, are preşented in Table 2-2. The national supply of carbon dioxide is estimated at 1.7 billion $\mathrm{m}^{3} / \mathrm{day}$, of which natural deposits are $2.2 \%$ and power plants are almost $90 \%$ of the total supply.

Natural deposits of carbon dioxide have been discovered in many parts of the United States. These known deposits, shown in Figure 2-1, are generally located in the southwestern United States and Appalachia, in close proximity to the petroleum basins amenable to EOR techniques. These natural deposits are typically capable of producing $83 \%-97 \%$ pure carbon dioxide, with reservoir pressures ranging from 600 to 3500 psig. These unique characteristics led to recent interest in known deposits by petroleum companies pursuing carbon dioxide EOR. Clearly, estimated natural deposits of 38.7 million $\mathrm{m}_{3}$ /day cannot meet estimated of peak EOR demands of over 120 million $\mathrm{m}^{3} /$ day.

Power plants are the single largest potential source for carbon dioxide. In contrast to natural deposits of carbon dioxide, power plants are widely distributed geographically, and the flue gas, at atmospheric pressure, contains up to $16 \%$ carbon dioxide. These impure, low-pressure carbon dioxide sources are distributed over wide regions and currently are not widely used by EOR operators. Utilization of flue gas in appreciable quantities at locations distant from the power plant requires collection, purification, compression, and transport of the carbon dioxide.

These additional requirements to obtain pure, high-pressure carbon dioxide result in large capital investments and operating cost. Most EOR project analyses limit estimates of carbon dioxide supply from flue gas power plants and by-product plants to within an $800-\mathrm{km}$ radius of the oil field (Anada et al. 1983). This constraint on the maximum economic transport distance lowers the national potential carbon dioxide supply for EOR to 654 million $\mathrm{m}^{3} /$ day or $38 \%$ of the total available supply.

Table 2-2. Total Potential $\mathrm{CO}_{2}$ Supply

\begin{tabular}{lc}
\hline \multicolumn{1}{c}{ Source } & $\begin{array}{c}\text { Amount } \\
\left(10^{6} \mathrm{~m}^{3} / \text { day }\right)\end{array}$ \\
\hline Natural $\mathrm{CO}_{2}$ deposits & 38.7 \\
Power plants & 1546.9 \\
Cement plants & 75.0 \\
Ammonia plants & 20.3 \\
Ethylene oxide plants & 1.57 \\
Ethanol plants & 0.4 \\
Natural gas processing & 7.21 \\
Hydrogen plants & 10.4 \\
Fluid catalytic cracker unit & 3.6 \\
regenerator & 1724.0 \\
Total &
\end{tabular}

Source: Anada et al. 1983. 

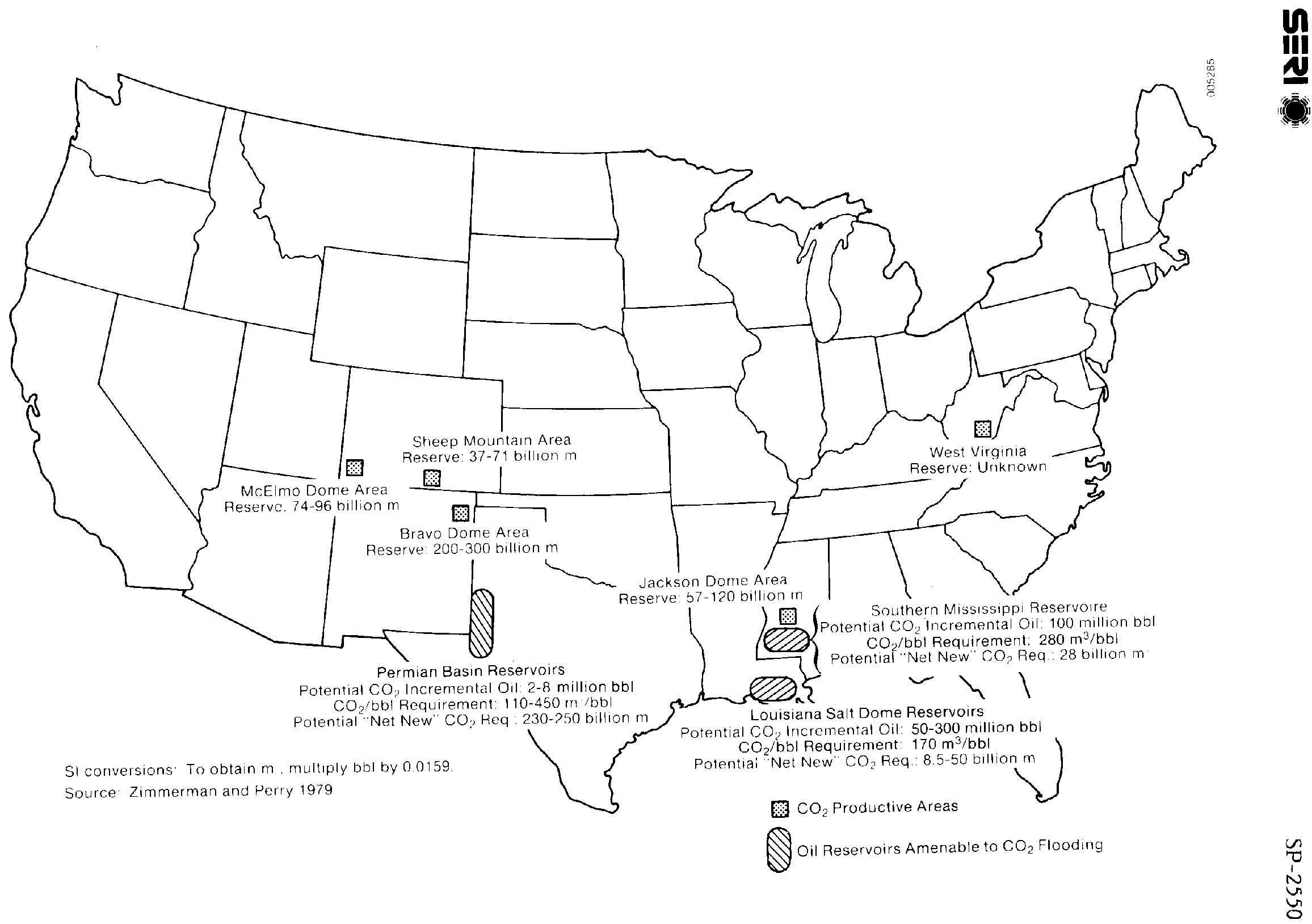
Natural deposits and flue gas sources represent the most feasible supply of carbon dioxide for enhanced oil recovery and also for microalgae production. Natural deposits, although a low percentage of the total supply, are capable of producing $\mathrm{CO}_{2}$ at required purity and pressure levels for both EOR and microalgae projects.

\section{Carbon Dioxide Supply in the Southwestern United States}

In their supply study, SAI estimates that over 617.6 million $\mathrm{m}^{3} /$ day of carbon dioxide are potentially available within $800 \mathrm{~km}$ of the geographical boundary of the Permian Basin (Anada et al. 1983). This supply estimate includes natural deposits of $\mathrm{CO}_{2}$, by-product $\mathrm{CO}_{2}$, and fluje gas streams from present and proposed plants. Presently about 360 million $\mathrm{m}^{3} /$ day of $\mathrm{CO}_{2}$ are available from existing plants in the region. Table 2-3 lists these supply sources along with additional supplies that would become available as a result of completion of proposed $\mathrm{CO}_{2}$ by-product plants in the region, about $80 \%$ of which is from power plant flue gas.

The SAI study concluded that low-cost carbon dioxide will be available only from large point sources such as coal- or gas-fired power plants. Carbon dioxide recovery technology based on extraction processes such as aqueous monoethanolamine (MEA) or

Table 2-3. Carbon Dioxide Supplies in the Permian Basin

\begin{tabular}{lc}
\hline \multicolumn{1}{c}{ Source } & $\begin{array}{c}\text { Amqunt } \\
\left(10^{6} \mathrm{~m}^{3} / \text { day }\right)\end{array}$ \\
\hline Natural deposits & $38.2^{\mathrm{a}}$ \\
Existing by-product plants & \\
Power plants & 285.1 \\
Cement plants & 14.8 \\
Ammonia plants & 5.5 \\
Ethylene oxide plants & 1.2 \\
Ethanol plants & .5 \\
Natural gas processing & 6.7 \\
Hydrogen plants & 1.5 \\
Refineries & 9.4 \\
Total existing by-product & 324.7 \\
& \\
Proposed by-product plants & \\
SNG plants & 88.5 \\
Power plants & 160.9 \\
Ethanol plants & 2.0 \\
Ethylene oxide plants & - \\
Refineries & 1.5 \\
Hydrogen plants & 1.2 \\
Total proposed & 254.2 \\
\hline a Includes natural CO 2 from Jackson Dome area. \\
Source: Anada et al. 1983.
\end{tabular}


potassium carbonate $\left(\mathrm{K}_{2} \mathrm{CO}_{3}\right)$ produces carbon dioxide from flue gases in a price range from $\$ 2.95$ to $\$ 8.82 / 10^{3} \mathrm{~m}^{3}$ ( $1984 \$$ ) depending on actual recovery rate, fuel type, and volume. Additional costs include compression, drying, and transport of $\mathrm{CO}_{2}$ from the point source to the user.

Therefore, the actual supply of $\mathrm{CO}_{2}$ from natural deposits and flue gas for EOR projects in the Southwest will depend on whether individual algae projects can secure sufficient quantities of low-cost $\mathrm{CO}_{2}$ and transport the carbon dioxide to the algae fuel farm at costs that are less than its value in EOR projects. Natural deposits cannot meet the entire projected $\mathrm{EOR}$ demand for $\mathrm{CO}_{2}$, but sources of by-product carbon dioxide may be required to sustain extensive $\mathrm{EOR}$ production through $\mathrm{CO}_{2}$ flooding methods. This potential shortage of carbon dioxide has stimulated a great deal of research on developing new technologies to extract carbon dioxide from flue gases.

Implications for Microalgae Development and Utilization

The preceding discussion of $\mathrm{CO}_{2}$ supply and demand in the southwestern United States indicates that while sufficient quantities of carbon dioxide exist to meet expected future EOR demand, there may be spot shortages due to high development costs at certain locations. Because of these costs, extensive research in further development of $\mathrm{CO}_{2}$ extraction from flue gas is being actively pursued by a number of companies.

Research in improving flue gas utilization for potential EOR projects also will aid in the economical recovery of $\mathrm{CO}_{2}$ for microalgae production. However, microalgae technology is expected to compete with these EOR projects for available sources of carbon dioxide. Within the Southwest, natural deposits will probably be developed just because of the desirable characteristics of the carbon dioxide from these sources. At present, three major natural $\mathrm{CO}_{2}$ deposits in the Southwest--McEImo Dome, Sheep Mountain, and Bravo Dome--have already been developed by petroleum companies. Their potential yield is 25 million $\mathrm{m}^{3} /$ day delivered to West Texas.

Economics of EOR dictate that beyond $800 \mathrm{~km}$, the cost of transporting $\mathrm{CO}_{2}$ is prohibitive. In addition, there is a question of scale in supplying $\mathrm{CO}_{2}$ to $\mathrm{EOR}$ fields: the large quantities of $\mathrm{CO}_{2}$ required for $\mathrm{EOR}$ must come from large power plants. While microalgae production requires more $\mathrm{CO}_{2}$ per barrel of oil produced, the total $\mathrm{CO}_{2}$ supply requirement is typically less than for large oil projects, and smaller power plants may be viable sources of $\mathrm{CO}_{2}$. Microalgae culture, which is more dispersed than concentrated oil projects, makes utilization of power plant $\mathrm{CO}_{2}$ feasible, especially if algal facilities are located near existing power plants.

A final consideration is the time frame for $\mathrm{CO}_{2}$ demand. EOR projects within the Permian Basin are being developed now for $\mathrm{CO}_{2}$ flooding. With an expected project lifetime of 20 to 30 years, many reservoirs will be depleted in the next 30 to 50 years. This depletion will weaken the demand by $\mathrm{EOR}$ projects for $\mathrm{CO}_{2}$ at the time microalgae development is expected to grow. As one $\mathrm{CO}_{2}$ consultant to the oil and gas industry reported, in about 30 years the Permian Basin is expected to be the world's largest underground source of $\mathrm{CO}_{2}$ (Petzet 1983).

In summary, there is good reason to believe that sufficient quantities of carbon dioxide will be available for microalgae production for liquid fuels. The supply sources for microalgae production are expected to be existing and planned power plants in the Southwest. In Arizona and New Mexico, existing power plant capacity is 13,939.5 MW, 
producing 122 million $\mathrm{m}^{3} /$ day of $\mathrm{CO}_{2}$. An additional $14 \%$ increase in capacity is planned over the next five to ten years (Energy Information Agency 1983). While EOR demands may utilize part of this supply between now and the year 2000 , depletion of oil reserves after 2000 should free large supplies of $\mathrm{CO}_{2}$ for microalgae production.

\subsection{Product Alternatives}

The major objective of the fuel products portion of this analysis is to determine how best to exploit the chemical composition of the algae to produce fuel products. The issue of comparing species product composition with fuel chemical requirements has been discussed in Feinberg (1984). Before examining the variety of potential fuel products, we first examine the properties that make an acceptable fuel product, both to producers and consumers. An assessment of the commercial fuel product network, both present and future, is presented since any promising fuel product will ultimately have to fit into that network. A brief characterization of the microalgae feedstock (as produced at the culture facility and fed to the fuel production facility) is presented to establish the basis for determining appropriate process requirements for converting algal chemical constituents into fuels.

\subsubsection{Functional Properties}

The first step in the evaluation of alternative fuel products is comparison with the physical, chemical, and functional properties of conventional fuels. Physical properties include the state of the fuel (solid, liquid, or gas), the density or specific gravity, the viscosity, various thermal properties such as boiling point and freezing point, and other complex properties such as cloud point, pour point, and flash point. The state of the fuel must be suited to its application: liquid fuels make the best fuels for transportation because they flow well, are stored easily, and provide a large amount of energy per unit volume. Gaseous fuels work well when no storage is available or necessary (e.g., pipeline systems), and solid fuels are best for stationary or semistationary applications (e.g., locomotives, power plants).

The density and viscosity of liquids and gases primarily affect the design of pumping and storage systems. Viscosity is a critical parameter for oxygenated diesel fuel substitutes (Ryan et al. 1984; Hill and Feinberg 1984). The thermal properties must be matched by the conditions of usage. For fuel products that are mixtures of components rather than pure substances, the mixture boils (or freezes) at a range of temperatures rather than at a discrete point but standards are usually set relative to some critical point in the range. In the case of diesel fuels, the point below which the fuel cannot be poured is the pour point, which is an indicator of performance; the fuel will not flow and burn efficiently below this point. Engine manufacturers generally do not recommend diesel fuel use below the cloud point--the point at which the fuel becomes cloudy and waxes start to form (Lane 1980). Cloud points are typically $6^{\circ}-10^{\circ} \mathrm{C}$ above the pour point. The flash point is the lowest temperature at which the fuel will ignite in the presence of an open flame. Among liquid fuels, gasoline has the lowest (best) flash point, about $-50^{\circ} \mathrm{F}$ $\left(-45^{\circ} \mathrm{C}\right)$, while methanol and ethanol have flash points that are of ten above ambient temperatures, $52^{\circ} \mathrm{F}\left(11^{\circ} \mathrm{C}\right)$ and $70^{\circ} \mathrm{F}\left(21^{\circ} \mathrm{C}\right)$, respectively.

In terms of chemical properties, the first and most important distinction between nonrenewable (fossil) fuels and those typically derived from biomass is that the constituents of petroleum, natural gas, and coal are all hydrocarbons--compounds composed entirely of carbon and hydrogen. In contrast, most biomass-derived fuels contain oxygen as well, 
1980). The validity of the cetane number for nonpetroleum-derived fuels is in question and is the subject of current research (Pryde 1984; Ryan et al. 1984). Diesel fuels marketed in the United States have cetane numbers ranging from 35 to 65 . Most manufacturers of truck and bus engines and of diesel locomotives specify use of fuels with a cetane number of 45 or above. Manufacturers of tractors and heavy construction equipment generally recommend a cetane number of 40 or above. The types of hydrocarbons that contribute to an increased cetane number are paraffins and naphthenes, the same compounds that would lower the octane number of a gasoline.

A detailed examination of fuels from biomass product is required to verify, if not identify, the valuable properties present. Octane enhancement is a good example of how a renewable fuel (ethanol) can improve a particular property of the nonrenewable fuel (gasoline) to which it is added. It has been hypothesized that a microalgae-derived fuel additive would improve the cetane number or the viscosity of diesel fuel. However, establishing if a microalgae-derived fuel is compatible with diesel fuel or could substitute for it requires rigorous evaluation of processing options and the product fuel.

\subsubsection{Overview of Fuel Product Network}

Of the three primary fuel sources in use today, petroleum provides the greatest variety of fuel products. Figure 2-2 shows schematically the fuel products produced from petroleum. Transportation by highway, railroad, and air are all represented, as are industrial and residential boilers and furnaces. As the feedstock characteristics or the product requirements change, the refinery can be adjusted to produce new product slates, such as more heating oil in the winter. Note that this figure includes refinery products only, not the network of petrochemicals.

Figures 2-3 and 2-4 illustrate the corresponding networks for natural gas and coal, the other two primary fuel product sources. Natural gas is a precursor of fewer products than petroleum, which is not surprising since it contains fewer components. Coal has a much greater variety of possible products, but only a few of them, notably benzene, toluene, and xylene (known collectively as BTX), plus coke and fuel gas, are utilized directly as fuels. The few entries under coal and natural gas contrast with the large variety of products derived from petroleum. The petroleum network is so tightly integrated that it is extremely hard to introduce new products into that market. If the ultimate objective is to displace an entire barrel of crude oil, then a whole set of substitutes, virtually an entire set of new products, must eventually be found. This makes the problem considerably more complex than simply developing new fuel products one at a time. However, alternative fuels with one attractive feature, for example ethanol and methanol with high octane values, have an opportunity for market entry, especially when they complement fuels derived from conventional sources and processes.

\subsubsection{General Characteristics of Crude Microalgae Feedstock}

To bridge the gap between the products produced by the microalgal culture facility and the various fuel production options, we must consider in detail the physical and chemical nature of the products produced by the culture system. Crude algae biomass is a thick slurry that consists of about $10 \%$ solids by weight and the components lipid, carbohydrate, protein, intermediates, and ash. Intermediates are low-molecular-weight substances that do not belong to the first three categories. Ash is the inorganic fraction, which does not contribute to the fuel value of the algal feedstock. 


\section{Microalgal Lipids}
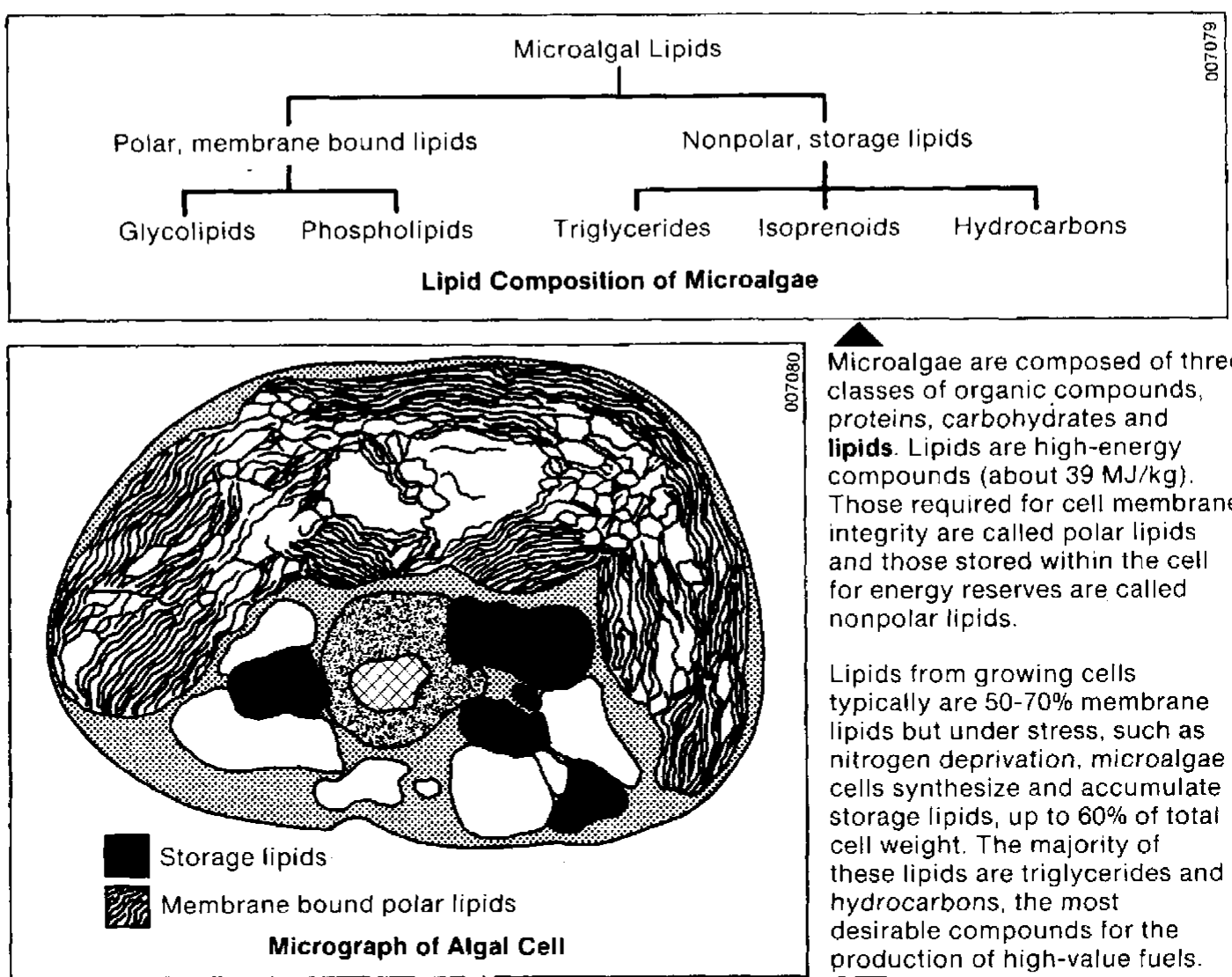

Microalgae are composed of three classes of organic compounds, proteins, carbohydrates and lipids. Lipids are high-energy compounds (about $39 \mathrm{MJ} / \mathrm{kg}$ ) Those required for cell membrane integrity are called polar lipids and those stored within the cell for energy reserves are called nonpolar lipids.

Lipids from growing cells typically are $50-70 \%$ membrane lipids but under stress, such as nitrogen deprivation, microalgae cells synthesize and accumulate storage lipids, up to $60 \%$ of total cell weight. The majority of these lipids are triglycerides and hydrocarbons, the most desirable compounds for the production of high-value fuels.

Storage lipids are promising feedstocks for fuel production due to their fundamental structural and chemical characteristics. Microalgae form vesicles (globules) of storage lipids within the cell that are more easily extracted chemically or mechanically than membrane lipids. Furthermore, storage lipids can be used, as extracted, as a diesel fuel substitute (triglycerides) or as a refinery blendstock (hydrocarbons). Additional processing can convert these storage lipids, especially triglycerides, into higher-value fuels such as ester fuel (a diesel fuel substitute) or gasoline.

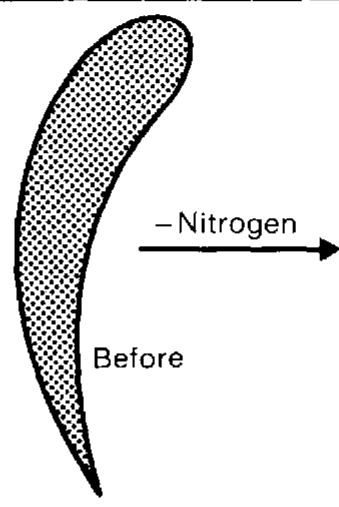

$25 \%$ Lipid

$40 \%$ Glycolipid

5\% Triglyceride 
Microalgal Lipids

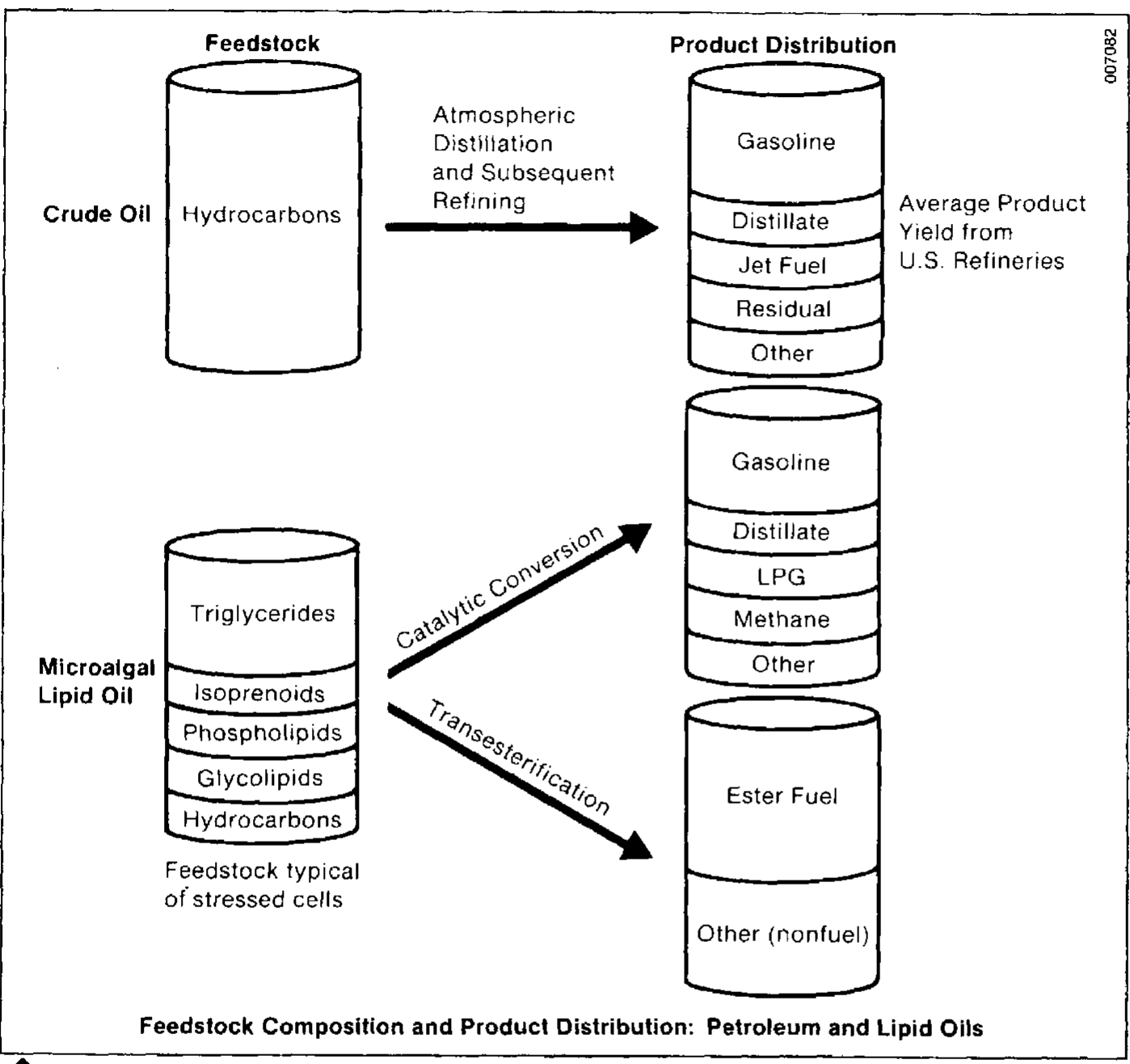

Microalgal lipid oils have been compared to crude petroleum but differ in their basic composition, required processing and final product yields. Proposed processing schemes yield gasoline and distillate fractions similar to average yields from petroleum. However, microalgae catalytic conversions are quite different from those used in oil refining. Transesterification of algal oils could yield up to $75 \%$ ester fuel.
The chemical composition of lipids is species specific and varies by the technique used to stimulate accumulation of storage lipids. Thus,

processing yields also can be expected to vary. Better definition of final product yields from microalgal lipid components is needed to define the desirable microalgae species characteristics and to optimize processing for the desired (highest value) fuel product distribution. 


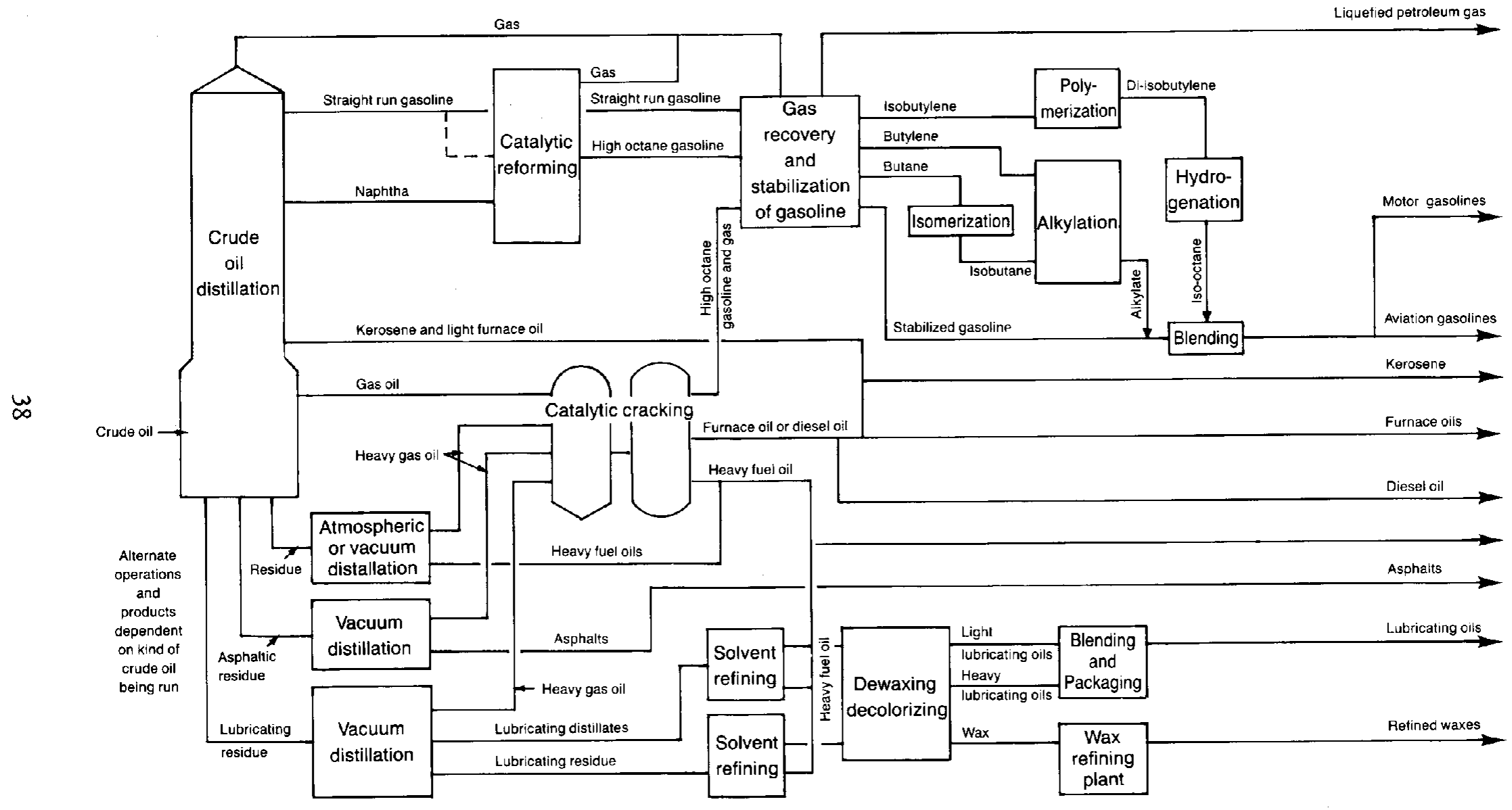

Figure 2-2. Generalized Overall Refinery from Crude Oil to Salable Products 
Wet Natural Gas

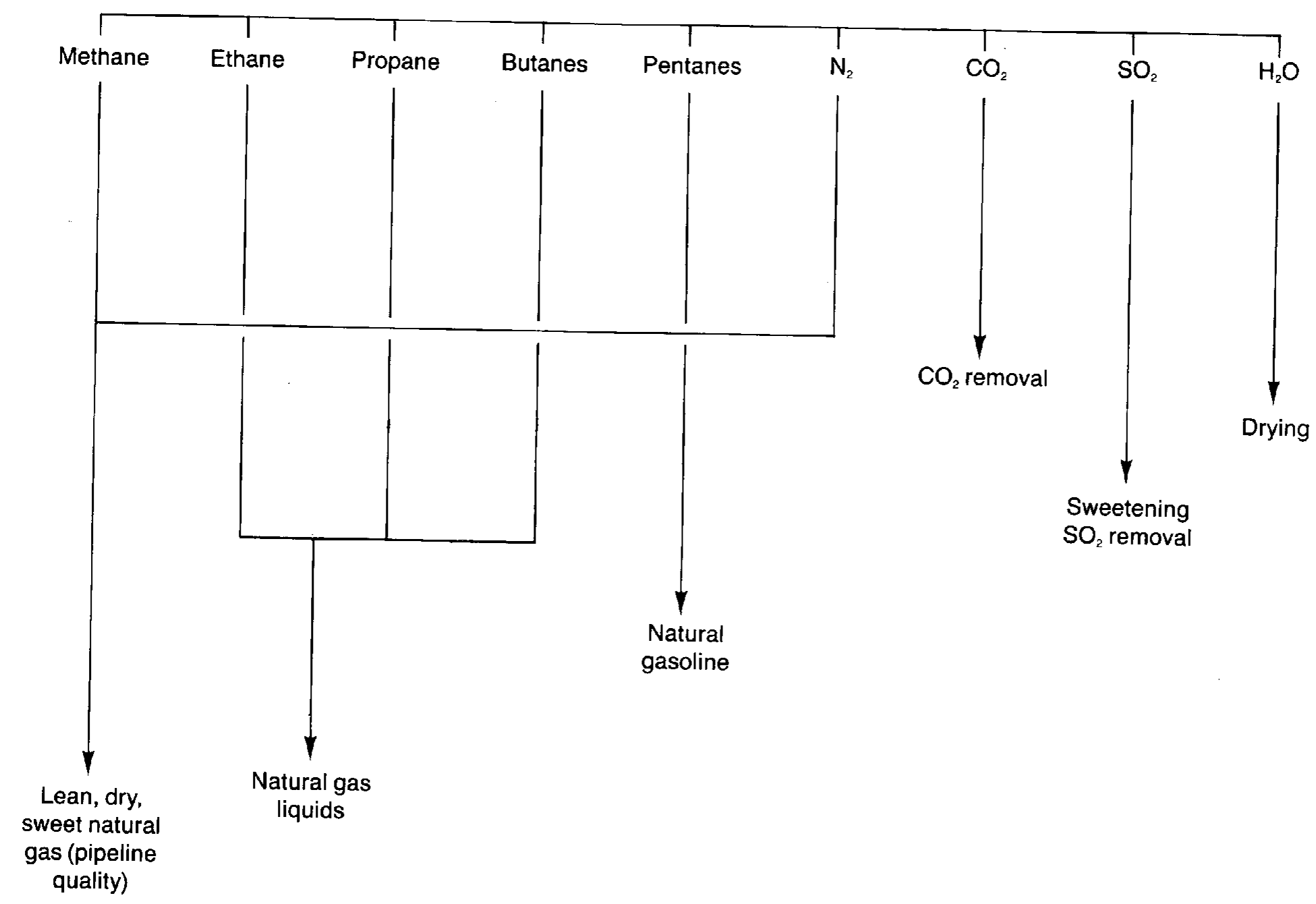

Figure 2-3. Network of Fuel Products from Natural Gas [Based on Shreve and Brink (1977)] 


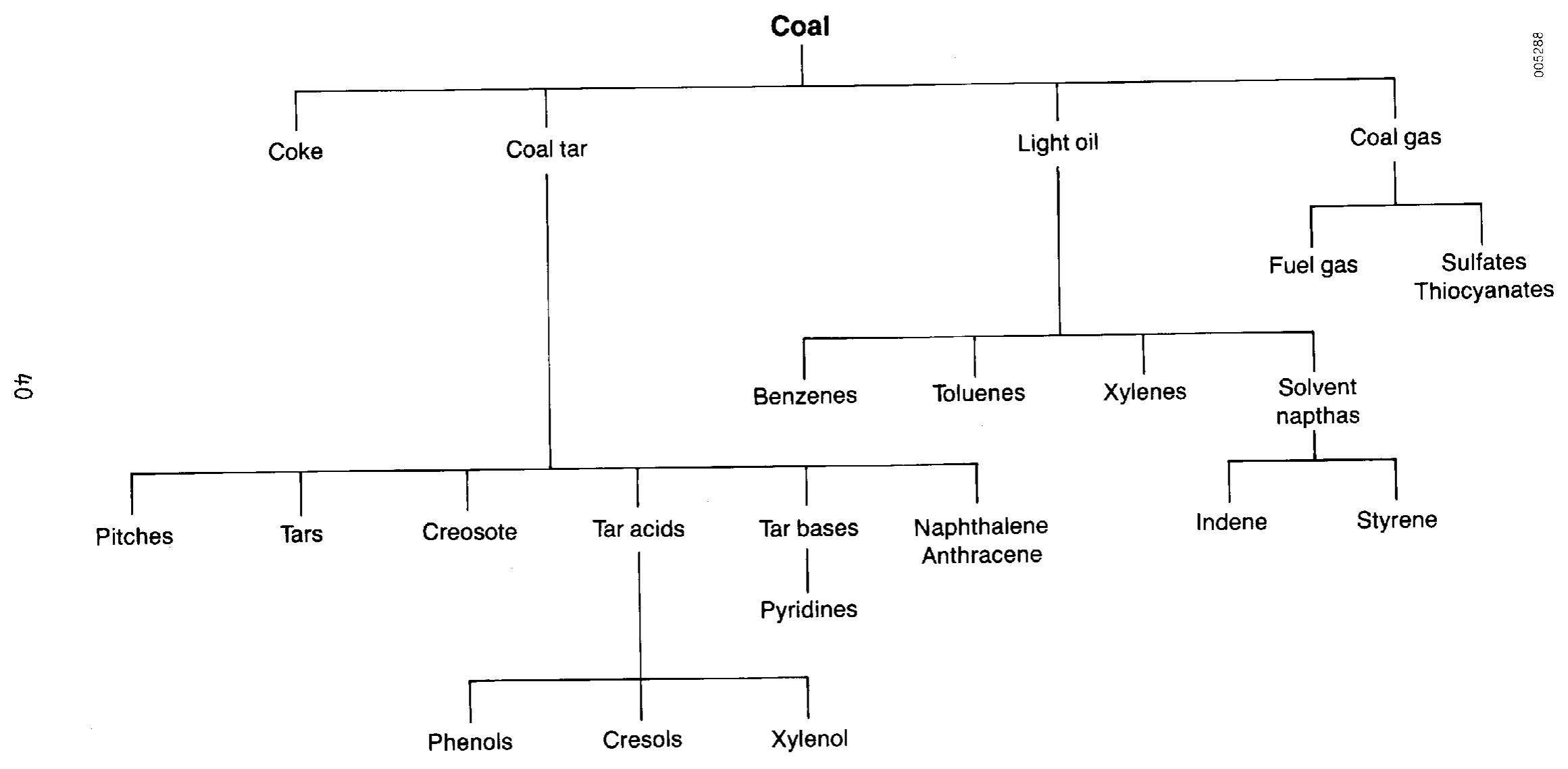

Figure 2-4. Network of Products from Coal

(Source: Shreve and Brink 1977) 
Table 2-5 shows the composition, specific energy, and gross energy content of a representative sample of algal biomass. The gross energy content is simply the product of the weight fraction and specific energy for each component. Lipids, while constituting only $30 \%$ of the total weight of the representative sample, contain over $46 \%$ of gross total energy content. Carbohydrates constitute $20 \%$ of the total weight but contain only $14 \%$ of the total energy. The calculated total gross energy content of the algae, $24.9 \mathrm{GJ} / \mathrm{t}$, is equivalent to a heat of combustion of $10,730 \mathrm{Btu} / \mathrm{lb}$. This compares favorably with coal whose heat of combustion varies from 7,000 Btu/lb for lignitic types to $14,000 \mathrm{Btu} / \mathrm{lb}$ for bituminous types (Perry and Green 1984). Additional characteristic types of the microalgal feedstocks are discussed below by specific conversion processes and final fuel products.

\subsubsection{Fuel Products from Lipids}

To examine fuel products from microalgae, we first must determine if crude algae feedstocks can be utilized directly in refineries, either alone or blended with crude petroleum. In the absence of detailed research to the contrary, the answer at the present time has to be no, primarily because of the presence of large amounts of oxygen. Algal biomass would be a poor blendstock because the oxygenates could react with the unsaturated hydrocarbons at the high temperatures used in crude distillation, causing polymerization or other undesirable reactions. Crude petroleum contains essentially no oxygen. The carbohydrate fraction of algae contains about 50\% oxygen and the lipid fraction about 10\%; thus, a feedstock with the chemical composition shown in Table 2-5 would contain about $13 \%$ oxygen. Now we will look at the utilization of the individual components of the algae and emphasize in this study the use of the highest energy fraction--the lipids. We will also look at alternative processes that utilize the carbohydrate fraction and the whole algae product.

\section{Pseudo Vegetable Oil (PVO)}

In recent years a significant amount of research and development has been directed toward the use of diesel oils refined from seed-bearing crops. These refined oils consist of the glycerol esters (trigiycerides) of a variety of fatty acids. Table 2-6 compares physical and chemical properties of triglycerides with those of diesel fuel. 'The fuel

Table 2-5. Composition and Energy Content of a Representative Sample of Crude Algae Feedstock

\begin{tabular}{lcccc}
\hline Component & $\begin{array}{c}\text { Weight } \\
\text { Fraction }\end{array}$ & $\begin{array}{c}\text { Specific } \\
\text { Energy } \\
(\mathrm{GJ} / \mathrm{t})\end{array}$ & $\begin{array}{c}\text { Gross Energy } \\
\text { Content } \\
(\mathrm{GJ} / \mathrm{t})\end{array}$ & $\begin{array}{c}\text { Percentage } \\
\text { of Total } \\
\text { Energy }\end{array}$ \\
\hline Lipid & 0.300 & 38.930 & 11.679 & 46.860 \\
Carbohydrate & 0.200 & 17.580 & 3.516 & 14.107 \\
Protein & 0.320 & 23.860 & 7.635 & 30.635 \\
Intermediates & 0.100 & 20.930 & 2.093 & 8.398 \\
Ash & 0.080 & 0 & $\frac{0}{24.923}$ & $\frac{100.000}{1.000}$ \\
Total & 1.00 & & & \\
\hline
\end{tabular}


Table 2-6. Suggested Standards for Vegetable Oil and Ester Fuels versus Diesel Oil Specifications

\begin{tabular}{|c|c|c|c|}
\hline \multirow{2}{*}{ Property } & \multicolumn{2}{|c|}{ Suggested Standards } & \multirow{2}{*}{$\begin{array}{l}\text { ASTM Specifications for } \\
\text { No. } 2 \text { Diesel Oil (D975) }\end{array}$} \\
\hline & Vegetable Oil & Ester Fuels & \\
\hline \multicolumn{4}{|l|}{ Physical } \\
\hline $\begin{array}{l}\text { Cetane number (minimum) } \\
\text { Cloud point (maximum) }\end{array}$ & $\begin{array}{l}30-40 \\
20^{\circ} \mathrm{C}\end{array}$ & $\begin{array}{l}30-40 \\
20^{\circ} \mathrm{C}\end{array}$ & $\begin{array}{l}40 \\
6^{\circ} \mathrm{C} \text { above } 1 / 10 \text { percen- } \\
\text { tile minimum ambient } \\
\text { temperature }\end{array}$ \\
\hline $\begin{array}{l}\text { Distillation temperatures, } 90 \% \text { point } \\
\text { minimum } \\
\text { maximum } \\
\text { Flash point (minimum) } \\
\text { Pour point (maximum) } \\
\text { Specific gravity, } 15 / 15^{\circ} \mathrm{C} \\
\text { Viscosity, } \mathrm{mm}^{2} / \mathrm{s} \text { at } 38^{\circ} \mathrm{F}\end{array}$ & $\begin{array}{l}>300^{\circ} \mathrm{C} \\
300^{\circ} \mathrm{C} \\
-5^{\circ} \mathrm{C} \\
0.910-0.930 \\
30-50\end{array}$ & $\begin{array}{l}>300^{\circ} \mathrm{C} \\
300^{\circ} \mathrm{C} \\
-5^{\circ} \mathrm{C} \\
0.8-0.9 \\
3-6\end{array}$ & $\begin{array}{l}282^{\circ} \mathrm{C} \\
338^{\circ} \mathrm{C} \\
52^{\circ} \mathrm{C} \\
- \\
- \\
1.9-4.1\end{array}$ \\
\hline \multicolumn{4}{|l|}{ Compositional } \\
\hline $\begin{array}{l}\text { Ash (maximum) } \\
\text { Free fatty acid (maximum) } \\
\text { Insolubles (maximum) } \\
\text { Iodine value } \\
\text { Moisture (Karl Fischer) (maximum) } \\
\text { Phosphorus (maximum) } \\
\text { Sulfur (maximum) } \\
\text { Volatile matter (maximum) } \\
\text { Water and sediment (maximum) } \\
\text { Wax (maximum) }\end{array}$ & $\begin{array}{l}0.05 \% \\
0.2 \% \\
0.001 \% \\
80-145 \\
0.2 \% \\
0.02 \% \\
-- \\
0.3 \% \\
--.02 \%\end{array}$ & $\begin{array}{l}0.05 \% \\
0.2 \% \\
0.001 \% \\
80-145 \\
0.2 \% \\
0.02 \% \\
-- \\
0.3 \% \\
-- \\
0.02 \%\end{array}$ & $\begin{array}{l}0.01 \% \\
-- \\
-- \\
-- \\
-- \\
-- \\
0.50 \% \\
-- \\
0.05 \% \\
--\end{array}$ \\
\hline
\end{tabular}

${ }^{\mathrm{a}}$ For example, the 10 th percentile minimum temperature for Northern Illinois is $16^{\circ} \mathrm{F}$ for November and $12^{\circ} \mathrm{F}$ for March, bracketing the winter period when there would be minimum use of tractors on farms.

Source: Pryde 1982.

value of refined vegetable oil is typically $5 \%$ lower and the boiling range is higher overall than diesel; the cetane number (subject to the uncertainties discussed earlier) is 35-40. The greatest difference between diesel fuel and vegetable oils occurs in viscosity, especially at low temperatures. Fuel system modifications (e.g., a fuel preheater or precombustion chamber) are required for reliable performance from vegetable oils.

The questions that need to be answered concern the similarities and differences between seed-oil lipids and alga! lipids (so-called pseudo vegetable oil or PVO). First, algal lipids are much more heterogeneous than the seed-oil lipids. Figure 2-5 shows the major components and typical amounts of five lipid fractions as recovered by a sequential solvent extraction technique. The chloroform fraction of the algal lipids is roughly equivalent to the seed oil, containing mostly triglycerides plus free fatty acid. The individual fatty acid components are quite different from those in the seed-oil lipids (Tornabene et al. 1983; Hill and Feinberg 1984), and the variation in chain length and degree of unsaturation are much greater for algal lipids. These factors make it difficult to characterize confidently algae-derived fuel products without more detailed investigations. At this time it is possible only to treat algal lipids as analogous to 


\begin{tabular}{|c|c|c|}
\hline Solvent & $\begin{array}{c}\text { Major components } \\
\text { of extract }\end{array}$ & $\begin{array}{c}\text { Range of } \\
\text { Typical content }\end{array}$ \\
\hline Hexane & Paraffins & $10 \%$ \\
\hline Benzene & $\begin{array}{c}\text { Olefins } \\
\text { Aromatics } \\
\text { Isoprenoids }\end{array}$ & $10-50 \%$ \\
\hline
\end{tabular}

Chloroform Mono-, di-, triglycerides $\quad 5-35 \%$

Fatty acids

Acetone $\quad$ Glycolipids $\quad 10-50 \%$

Methanol Phospholipids 5-40\%

Figure 2-5. Major Lipid Fractions as Recovered via Sequential Solvent Extraction (Source: Tornabene et al. 1983)

vegetable oil and evaluate existing vegetable oil processes to determine the costs and yields of PVO from lipids.

\section{Ester Fuel}

Another option for using algal lipids offers some improvements over PVO in critical areas. This option is the transesterification of the triglycerides: the interchange of the glycerol (the component exploited in PVO fuels) for methanol or ethanol, thereby producing methyl or ethyl esters. This process has also been utilized with seed oils, so the adaptation to algal lipids is straightforward. Important properties of the resulting socalled ester fuel are discussed by Hill and Feinberg (1984) and are listed in Table 2-6. Although the fuel value is about $10 \%$ lower than diesel fuel $15 \%$ lower than vegetable oils), ester fuel has significant advantages over seed oil in both viscosity and cetane number. The distillation curve (boiling range) is between those of diesel and vegetable oil. The only disadvantage of the ester fuel is that the cloud point is estimated to be between $0^{\circ}$ and $10^{\circ} \mathrm{C}$. Use of ester fuel at or near these temperatures would require some fuel or fuel line preheating, although a diesel-ester blend (as well as a diesel and seed-oil blend) would minimize this requirement.

\section{The M-Gas Process and Its Potential}

The high energy content of hydrocarbons, plus their greater familiarity to petroleum refiners, has led to a recent interest in the conversion of oxygenates to hydrocarbons. In particular, methanol has been evaluated extensively as a candidate because it can be produced from coal. Mobil Research and Development Corporation has done extensive 
work in developing thermal-catalytic processes that convert methanol to light hydrocarbons in the gasoline range (Voltz et al. 1976). Mobil researchers have also investigated similar processes using other feedstocks such as corn oil, castor oil, jojoba oil, and latex (Weisz et al. 1979), which all resemble microalga! lipids.

A typical product distribution from corn oil is shown in Figure 2-6. The distinguishing products are paraffins, olefins, and aromatics, with the rest identified as nonaromatics. Of particular interest are the aromatics with carbon numbers 6 through 8; these compounds, benzene, toluene, and the xylenes (BTX), represent octane-enhancing refinery streams currently used in both leaded and unleaded gasolines. The other significant products are in the liquefied petroleum gas (LPG) range and include mostly paraffins and some olefins. Both castor oil, which contains hydroxy-substituted fatty acid chains, and latex, a polymer of isoprene (2-methyl,1,3-butadiene), produced an even larger BTX fraction. The similarity between these feedstocks and the algal lipids provides encouragement, although the algal lipids contain more polyunsaturated acids than the corn oil, and their isoprenoids may be ring compounds rather than straight chained as in latex. These differences affect reactivity, yields, and product distribution. Nonetheless, algal lipids appear to be good candidates for catalytic conversion to high-value liquid fuels.

More research with Mobil's or a similar catalyst would be required to verify the potential of algal lipids as feedstocks. Drying, extraction of the reactive fraction (triglycerides, etc.), and other preprocessing might be required to prepare the harvested algae for such a process.

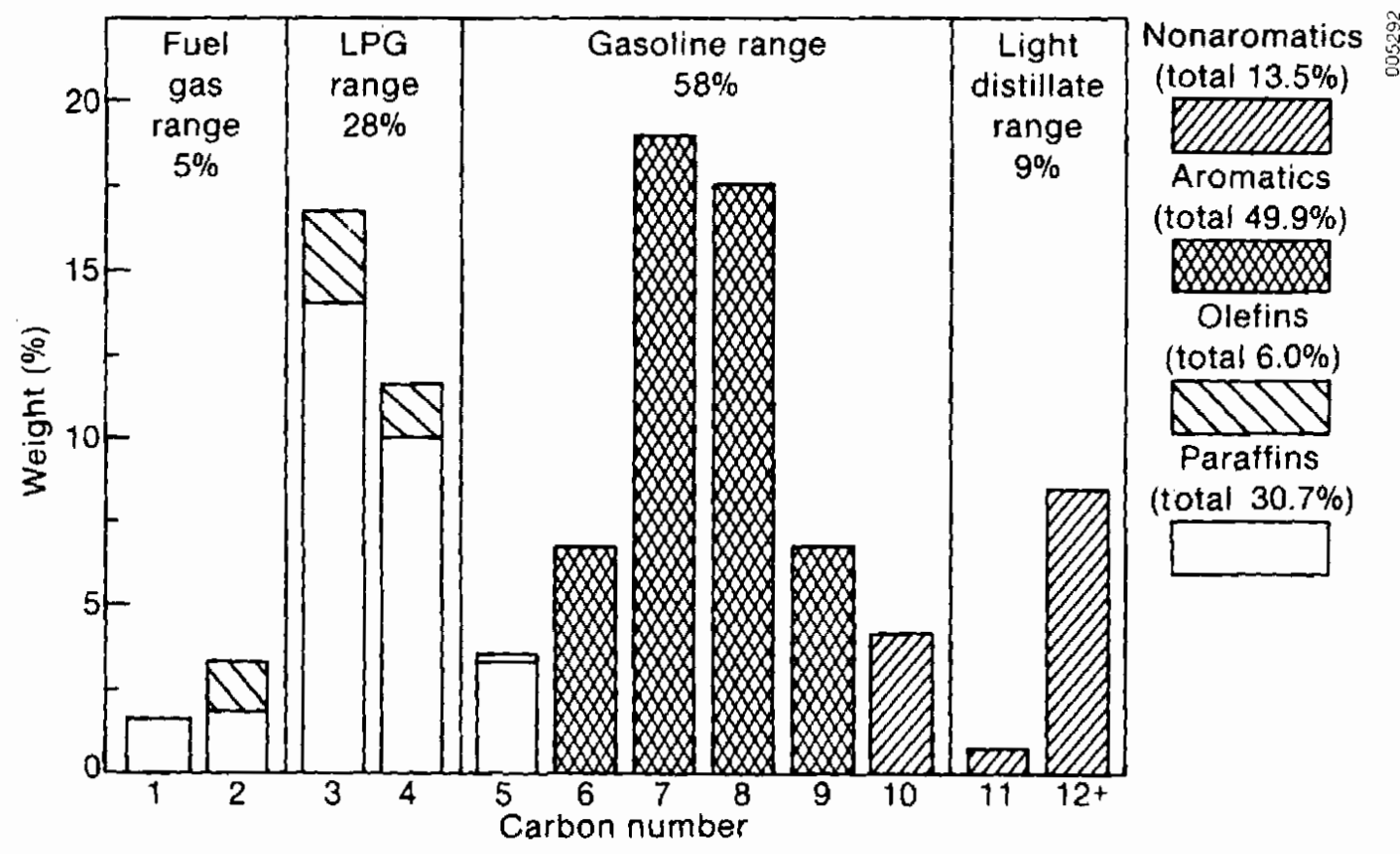

Figure 2-6. Product Distribution from Catalytic Conversion of Triglycerides (Corn oil at $450^{\circ} \mathrm{C}$ ) (Source: Weisz et al. 1979) 


\subsubsection{Other Products and Processes}

In addition to lipids, algae contain carbohydrates and protein that can be used in more developed (relative to lipid-based) processes to produce liquid or gaseous fuels. Two relatively well developed technologies are chosen as alternative processes for producing fuels from microalgae.

\section{Methane}

Anaerobic digestion is the one known process by which most of the algal cell mass is converted into a single fuel product. Methane, the primary constituent of natural gas, is widely used as both a fuel and as a chemical feedstock. Since under normal conditions it is a gas and therefore bulky to handle, its use as a transportation fuel has been limited. However, natural gas boilers and combustion turbines are in wide use. Liquefied natural gas (LNG), with increased density, has begun to be used as a transportation fuel.

An anaerobic digester contains synergistic microbial populations to convert a variety of organic substrates to methane and carbon dioxide. Thus algal organic compounds (lipid, protein, or carbohydrate) can be converted to methane.

Few problems are expected in the adaptation of the microalgae feedstock to anaerobic digestion. Microalgal feedstock digestion is already being studied (Wagener 1981; Samson and Leduy 1983), and macroalgae are also promising feedstocks for methane (GRI 1983). The major technical concern is the continuing development of more efficient digesters. The analysis presented here was based on a plug-flow reactor design, which has been studied widely. Other digester designs, in particular those employing fluidized beds, might offer better conversion efficiencies and shorter residence times.

The primary digester product, usually referred to as biogas, is not pure methane, but contains large amounts (typically 40\%) of carbon dioxide, plus trace amounts of water and hydrogen sulfide. Water, sulfur, and nitrogen must be removed prior to use, but removal of the $\mathrm{CO}_{2}$ is optional. If the gas is to be burned on site, $\mathrm{CO}_{2}$ removal is not required but the gas will have a lower Btu content per cubic meter, so a larger volume of gas is required compared to natural gas. Direct use of this medium-Btu gas, as it is commonly called, is still probably cheaper than $\mathrm{CO}_{2}$ removal in small applications. However, when large volumes of gas are sold to a pipeline company or gas utility, $\mathrm{CO}_{2}$ removal will be required. This can be accomplished via several gas absorption processes; water, sodium carbonate, and ethanolamines are the most common absorbents. Removal of the carbon dioxide from microalgae-based biogas would create the potential for $\mathrm{CO}_{2}$ recycle into the culture system, while producing a high-Btu gas suitable for either pipelines or further chemical processing.

\section{Ethanol}

Ethanol is the highest-volume product, fuel or otherwise, produced commercially by biological fermentation. In the beverage and fuel industries, the primary substrate is starch from corn or other grains, although any carbohydrate source is suitable for conversion to sugars and subsequently to ethanol.

Algal protein could be separated from the fermentable sugars and recovered as a salable food or feed product similar to soybean meal, although species-specific research is required to characterize the protein product. Also, the polysaccharides in microalgae are in the form of starch, so the sugars are accessible for yeast fermentation. The major 
issues concerning ethanol production from microalgae are the maximum carbohydrate content that can be achieved and the portion of the carbohydrate that could be converted to fermentable sugars and then to ethanol.

\section{Thermochemical Conversions}

It is likely that fuel products other than those discussed here may be produced from microalgae. Most of the microalgal research to date has concentrated on their production and growth, and maximizing production of one fraction (i.e., lipids). Little attention has been paid to other potential products. The opportunities are great for improvements and even breakthroughs. For example, the entire algal mass, with a suitable catalyst, could be hydrogenated to a mixture of paraffins and olefins in the 16-22 carbon range, and either blended directly into crude oil or used as a cracking stock. This reaction has been investigated using a strain of Chlorella pyrenoidosa (Chin and Engel 1981). Other conversions are also possible, most notably a thermochemical conversion to produce higher alcohols, which have octane-enhancing properties of ethanol and methanol but pose fewer water solubility and phase separation problems. At this point there are insufficient data to analyze these processes further.

\subsubsection{Summary}

Table 2-7 summarizes estimated yields of five fuel products from microalgae with the composition shown in Table 2-5. These comparisons provide an overview of the various fuel options. A complete evaluation of the feasibility of producing these fuels from microalgae requires an estimation of feedstock production costs, which is presented in the next section, and conversion costs, which is presented in Section 4.0. 
Table 2-7. Surnmary of Reference Processing Product Yields ${ }^{\mathrm{a}}$

\begin{tabular}{|c|c|c|c|c|c|}
\hline & PVO & $\begin{array}{c}\text { Ester } \\
\text { Fuel }\end{array}$ & Gasoline & Methane & Ethünol \\
\hline Major algal component & Lipid & Lipid & Lipid & All & Carbohydrate \\
\hline Fraction of total algae & 0.30 & 0.30 & 0.30 & -- & 0.20 \\
\hline Fraction available for conversion & 0.40 & 0.40 & 0.60 & 0.90 & 0.65 \\
\hline Efficiency of utilization of major component & 1.00 & 0.98 & $0.90^{b}$ & 0.80 & $0.855^{\mathrm{C}}$ \\
\hline Major production $(t / t$ algae) & 0.120 & 0.112 & 0.085 & -- & 0.057 \\
\hline Common unit/t algae & $34.4 \mathrm{gal}$ & $33.5 \mathrm{gal}$ & $30.7 \mathrm{gal}$ & $14.00 \mathrm{GJ}$ & $18.99 \mathrm{gal}$ \\
\hline Product energy content $(G J / t)$ & 39.5 & $36.90^{\circ}$ & 43.86 & -- & 26.7 \\
\hline Main energy products (GJ/t algae) & 4.74 & 4.14 & 3.74 & 14.00 & 1.52 \\
\hline Total energy available (GJ/t algae) & 24.92 & 24.92 & 24.92 & 24.92 & 24.92 \\
\hline Recovery efficiency (major product) & $19.0 \%$ & $16.6 \%$ & $15.0 \%$ & $56.2 \%$ & $6.1 \%$ \\
\hline
\end{tabular}

${ }^{\mathrm{a}}$ Algal feedstock composition as shown in Table 2-5.

${ }^{\mathrm{b}} \mathrm{GJ}$ basis hydrolysis efficiency $\times 0.95$ fermentation efficiency.

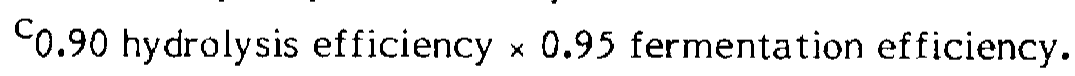




\subsection{MASS CULTURE ECONOMICS}

An important part of a technical and economic evaluation of mass culturing microalgae is estimating the costs associated with the production process. This section discusses the development of these cost estimates. Following a review of major system components and design issues we examine available physical and economic data for individual components. Finally, we develop a reference microalgae production system and a cost estimate. Cost estimates were facilitated by the use of the Algal Production and Economic Model developed by Hill (1984), which allows easy variation of key components to identify cost sensitivities, the starting point for establishing research targets.

\subsection{Process Design Economics}

\subsubsection{Pond Configurations and Cost Factors}

The Algal Production and Economic Model (APEM) has as its basic production unit a pond configuration similar to that described by Benemann et al. (1982). The facility is composed of individual modules or ponds arranged to facilitate the use of centralized distribution systems for nutrient and water additions and for harvesting. Between $15 \%$ and $20 \%$ of the total land area is dedicated to on-site nonproductive uses such as support facilities. Cost estimates are developed for the major subsystems of the facility and for individual modules using construction cost estimates reported by Benemann et al. (1982) and additional cost estimates from a variety of sources.

Civil construction costs, exclusive of lining for the growth ponds are estimated to be $\$ 8450 /$ ha $(1984 \$)$. These costs include site preparation and surveying, laser grading, and primary berm construction. Channel dividers in the ponds, modified from Benemann's (1982) secondary berm design, are thin plastic fences, which are less expensive than secondary berms and take up less surface area. In addition, each module is lined with a granular cover over a clay bed at a cost of $\$ 5000 /$ ha. At many sites, soil characteristics are conducive to compacted earth, granular-bed channels. However, with extensive adoption of the technology, developers may encounter soils of high permeability, and more expensive plastic or polymer liners that could cost two to three times as much to install may be required to prevent excessive water loss.

The circulation system for each module employs conventional paddlewheels at an estimated cost of $\$ 2500 /$ ha. Alternative mixing systems differ in their hydraulic mode and pump efficiency. For example, the mixing system efficiency is $59 \%$ for a paddlewheel but only $27 \%$ for an air lift pump. Either system may be installed at the $\$ 2500 /$ ha cost estimate, and the choice of mixing systems depends on the desired properties and module design. While more expensive to operate, the air-lift system can also distribute $\mathrm{CO}_{2}$ into the culture medium. The reference system for this study assumes paddlewheel mixing.

Capital costs for the carbon dioxide sparger system and the water/nutrient distribution system within the facility are based on the estimates presented by Benemann et al. (1982) designed for the distribution of dilute carbon dioxide flue gas but adjusted for the use of pure $\mathrm{CO}_{2}$. Exclusive of the capital costs for the large concrete supply pipe, Benemann's cost estimates include $\$ 4.6$ million of fixed costs for each 2000 -acre facility and $\$ 46,000$ in incremental costs for each module. The water/nutrient distribution system requires $\$ 420,000$ of fixed costs and incremental module costs of $\$ 21,000$. 
The prices of major nutrients are assumed to be contracted prices. These nutrients include ammonia gas, superphosphate, and potassium muriate. The quantity required for each nutrient is based on total consumption determined by algal productivity requirements, outgassing losses, and blowdown losses. Nutrients that are not incorporated into algal cell mass but remain in the media may be recycled to the extent that the culture salinity tolerance is not exceeded; the amount recycled is an input parameter determined exogenously.

\subsubsection{Harvesting Options}

Harvesting cost estimates are based on three harvester subsystems commonly employed in algal dewatering schemes: a microstrainer, a centrifuge, and a belt filter. The microstrainer and belt filter systems are used as the first-stage systems; the centrifuge used as a second stage brings the product stream to the design requirement of at least $10 \%$ solids.

The concentration factors for the harvester systems are 10,70, and 150 for the microstrainer, belt filter, and centrifuge respectively (Mohn 1980; Dodd 1984), and energy consumption rates are $0.2,0.88$, and $1.2 \mathrm{kWh} / \mathrm{m}^{3}$ of throughput, respectively. Capital cost estimates represent the largest commercial system available for each harvester. The effluent from the ponds is divided into individual streams that are capable of being handled by the harvesters. The microstrainer and belt filter are capable of handling $13 \mathrm{~m} /$ minute, whereas the centrifuge has a design limitation of $3.8 \mathrm{~m}^{3} /$ minute.

\subsubsection{Production Labor}

Production labor was estimated at $\$ 1345 /$ ha of total facility size (Benemann et al. 1982). For a 1000-ha facility, the labor estimate assumes annual salary requirements for 1 plant engineer, 4 shift supervisors, 20 pond operators, 8 secondary harvesting operators, 8 processing operators, and 2 laboratory personnel. Additional overhead expenses of $75 \%$ of direct labor are also included in final production labor costs for the facility. These small labor requirements are consistent with a highly automated facility, the basis for all design examined in this report.

\subsubsection{Energy Requirements}

Energy consumption for the mass culture facility is divided among harvesting, pumping, and mixing systems. Energy consumption for mixing and pumping is calculated from total head loss. Energy consumption for harvesting is based on throughput to the harvesters using the energy consumption estimates of Mohn (1980). Total energy used for mixing was calculated from Manning's equation for head loss in channel flow systems.

Energy used in pumping the medium from the modules to the harvester subsystems, recycled water from harvesters to the headbox, makeup water, and disposal water are estimated from assumed head losses for a nearly flat site. Each major pumping routine is summed for the entire facility based on flow rates appropriate for maintaining mass balances among all processes. Based on horsepower requirements for each pumping routine, energy consumption is determined by the number of days the facility operates. Energy costs are based on average energy costs to large industrial users in the Southwest. 


\subsubsection{Resource Supplies}

\section{Water}

Microalgae culture in this analysis is based on utilization of low value saline waters that are presumed to be abundant in the southwestern United States. The high salinity precludes their use for potable or irrigation water, and enhanced oil recovery (EOR) projects represent the only demand for these resources--carbon dioxide or other inert gases and saline water are injected into low-pressure wells to force entrapped oil to the surface. Sufficient saline water for this purpose is found in conjunction with the oil or gas, so EOR will not compete for water from saline reservoirs.

Data collected on saline water resources typically reflect only the interests of the oil and natural gas industry and are insufficient for ascertaining the quantity and quality available for microalgae recovery. Well logs record the occurrence and chemical composition of saline water found near oil and gas reserves. However, well log data do not include estimates of how much of the saline water resource can be pumped to the surface, since historically this water is of little value.

To estimate the available quantity of saline water for use above ground, additional data on area yield is required. Area yield estimates characterize the reservoir in terms of pumping capacity and the impact on adjoining (perhaps potable) water reservoirs and give estimates of sustainable pumping limits and the total available water resource. Without detailed pumping data it is not possible to estimate area yields, and the quantity of saline water available for use in microalgae culture remains uncertain.

The water costs are based on a delivered cost $\left(\$ / \mathrm{m}^{3}\right)$ to supply a microalgae facility with sufficient water as makeup to control culture salinity. Because of the uncertainty in the design and scope of a water supply project that would be capable of supplying the necessary water to a mass culture facility, a range of water costs was estimated based on analyses conducted by Maxwell et al. (1984). Area-specific estimates of drilling costs and delivered force await more extensive evaluation of the saline water resources of the Southwest.

\section{Carbon Dioxide}

Carbon supply costs are estimated initially on the basis of $\mathrm{CO}_{2}$ obtained from a power plant. This offsite facility is assumed to use a potassium carbonate extraction system with purification and compression; the $\mathrm{CO}_{2}$ would be transported via pipeline to the mass culture facility. This supply system is costed based on a functional relationship between daily supply requirements, transport distance, and delivered average unit cost for the carbon dioxide.

Once the mass culture facility's daily requirement for $\mathrm{CO}_{2}$ is determined, the delivered cost of $\mathrm{CO}_{2}$ is determined by calculating the 30-year amortized cost of the potassium carbonate extraction system and the amortized cost of a pipeline to transport the $\mathrm{CO}_{2}$ to the culture facility. The cost functions for these parameters are based on data developed for determining the delivered cost of $\mathrm{CO}_{2}$ to enhanced oil recovery fields (Anada et al. 1983). The initial design serves as a basis for completing a full production configuration but is subject to uncertainty, so we examine later alternative $\mathrm{CO}_{2}$ delivery schemes and the range of costs they imply. 


\subsection{Analytical Method for Evaluation}

\subsubsection{Model Description}

The Algal Production and Economic Model (APEM; Hill 1984) consists of a series of linked physical and economic relationships representing the major aspects of microalgae cultivation, production costs, and economics. While a comprehensive treatment of all factors affecting cultivation and cost must await resolution of many issues, the model was designed and has evolved to provide sufficient detail in critical areas of microalgae production technologies.

A schematic of the APEM is presented in Figure 3-1 (see Appendix A for the entire model). The main computer model contains the algal production system, which specifies the appropriate data for all the other models. Because the operations and production levels within the culture system determine the levels of resource input requirements and ultimately establish production output levels from the facility, knowledge of the basic biology of the system is most important.

The production model reflects a steady-state, continuous production calibrated to empirical data from field experiments. Gross productivity is determined by specifying culture depth, density, and detention time (or the inverse of dilution rate). Then, based on the assumed chemical composition of the microalgae and the size of facility, data on flow rates, nutrient requirements, and product yields are calculated according to deterministic, functional relationships.

Resource inputs are determined by productivity levels. Requirements for carbon, nitrogen, potassium, and phosphorus are based on inputs required to maintain growth in the culture system. Gross nutrient demand includes the deficits resulting from blowdown for culture salinity control and culture outgassing losses; this demand can be partially offset by nutrients present in the source water. The amount of water required is a function of water losses due to culture surface evaporation, blowdown, and harvesting.

The culture density and rate of harvest from the system determine the harvester subsystem requirements. Nominally, a product requirement of $10 \%$ solids is used to determine the extent of harvester concentration required. Harvester flow rates determine the number of first-stage harvester systems--microstrainers or belt filters--required. The product stream from these harvesters is concentrated to the design value (10\% solids) by centrifuges.

\subsubsection{Overview of Reference Values and Ranges for Model Parameters}

\section{The Reference Case}

In order to explore the critical issues of mass cultivation of microalgae for fuel production, a reference (base) case was established. This reference case, defined as a specific set of input parameters to the model (Table 3-1), reflects a consensus by SERI researchers and other experts on values that might be expected if mass cultivation of microalgae were undertaken today in the southwestern United States. A consensus was difficult to reach on several parameters because data are lacking. In such cases, one reference value was chosen from the range of possible values. The complete reference case represents neither a minimum nor a maximum of the potential for microalgae but rather a set of values from the range of possible results. The reference system, based on 


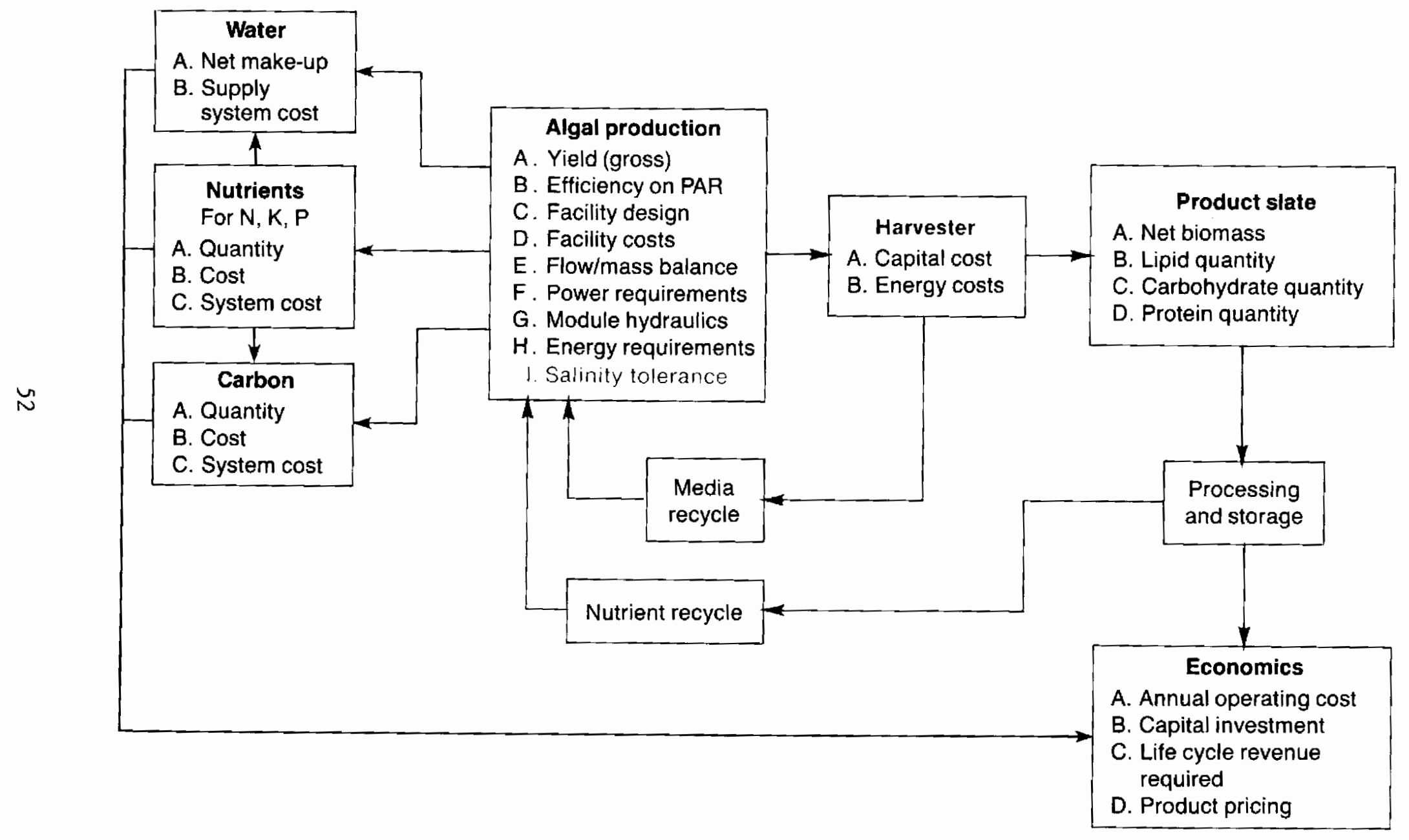

Figure 3-1. Overview of Algal Production and Economic Model (APEM) 
Table 3-1. Parameter Ranges Identified for Microalgae

Systems (1984\$)

\begin{tabular}{lll}
\multicolumn{2}{c}{ Range } & \\
\hline Low & Reference & High \\
\hline
\end{tabular}

Category A: Resource Parameters

Evaporation $(\mathrm{m} / \mathrm{d})$

$\begin{array}{ccc}0.002 & 0.0035 & 0.01 \\ 2.5 & 25 & 35 \\ 0 & 13 & 96 \\ 0 & 0.5 & 1 \\ 8 & 46 & 400 \\ 10 & 200 & 420 \\ 1000 & 1245 & 1500 \\ 0.040 & 0.065 & 0.10 \\ 0.05 & 0.067 & 0.20 \\ 165 & 185 & 205 \\ 225 & 254 & 280 \\ 80 & 92 & 100 \\ 0 & 0 & 100 \\ 0.13 & 0.13 & 0.13\end{array}$

Salinity of source water (g TDS/L)

Nitrogen in source water $\left(\mathrm{g} / \mathrm{m}^{3}\right)$

Phosphorus in source water $\left(\mathrm{g} / \mathrm{m}^{3}\right)$

Potassium in source water $\left(\mathrm{g} / \mathrm{m}^{3}\right)$

Carbon in source water $\left(\mathrm{g} / \mathrm{m}^{3}\right)$

Land cost (\$/ha)

Energy cost $(\$ / k W h)$

Water cost $\left(\$ / \mathrm{m}^{3}\right)$

Ammonia cost $(\$ / t)$

Superphosphate cost $(\$ / t)$

Potassium cost $(\$ / t)$

Distance to $\mathrm{CO}_{2}$ source $(\mathrm{km})$

$\mathrm{CO}_{2} \operatorname{cost}(\$ / \mathrm{m})$

0.13

70

86

90

Effective culture area ( $\%$ of total size)

30

10

5

Module size (ha)

10

20

40

Channel width $(\mathrm{m})$

6

30

60

Channel roughness (Manning's " $n$ ")

0.023

0.025

0.03

Depth of culture $(\mathrm{m})$

0.10

0.15

0.20

Carbon in medium $\left(\mathrm{g} / \mathrm{m}^{3}\right)$

7.5

12

25

Nitrogen in medium $\left(\mathrm{g} / \mathrm{m}^{3}\right)$

0.14

0.28

14

Phosphorus in medium $\left(\mathrm{g} / \mathrm{m}^{3}\right)$

0.31

0.62

31

Potassium in medium $\left(\mathrm{g} / \mathrm{m}^{3}\right)$

5

Carbon losses $\left(\mathrm{g} / \mathrm{m}^{3} / \mathrm{d}\right)$

25

0

0.1

400

Nitrogen losses $\left(\mathrm{g} / \mathrm{m}^{3} / \mathrm{d}\right)$

0

Mixing velocity $(\mathrm{m} / \mathrm{s})$

0.05

0.1

0.2

0.3 
Table 3-1. Parameter Ranges Identified for Microalgae

Systems (1984\$) (concluded)

\begin{tabular}{|c|c|c|c|}
\hline & \multicolumn{3}{|c|}{ Range } \\
\hline & Low & Reference & High \\
\hline Mixing system efficiency (\%) & 30 & 65 & 75 \\
\hline Harvester solids removal (\%) & 50 & 90 & 98 \\
\hline $\begin{array}{l}\text { Harvester type } \\
\text { First stage } \\
\text { Second stage }\end{array}$ & \multicolumn{2}{|c|}{$\begin{array}{l}\text { Microstrainer } \\
\text { Centrifuge }\end{array}$} & $\begin{array}{l}\text { Belt filter } \\
\text { Eentrifuge }\end{array}$ \\
\hline \multicolumn{4}{|l|}{ Category C: Biology Parameters $^{\mathrm{a}}$} \\
\hline Ash content ( $\%$ dry wt) & 8 & 8 & 8 \\
\hline Lipid content ( $\%$ dry wt) & 20 & 30 & 60 \\
\hline Carbohydrate content ( $\%$ dry wt) & 49 & 20 & 9 \\
\hline Protein content ( $\%$ dry wt) & 13 & 32 & 13 \\
\hline Intermediate content ( $\%$ dry wt) & 10 & 10 & 10 \\
\hline Salinity tolerance (g TDS/L) & 10 & 35 & 120 \\
\hline Phosphorus cell content ( $g / g$ dry wt) & 0.0012 & 0.007 & 0.023 \\
\hline Growing season $(d)$ & 200 & 250 & 365 \\
\hline Photosynthetic efficiency on PAR (\%) & 4 & 5 & 16 \\
\hline Depth $(m)$ & 0.10 & 0.15 & 0.30 \\
\hline Detention time $(d)$ & 7 & 7 & 3 \\
\hline Density (mg/L) & 500 & 600 & 1000 \\
\hline \multicolumn{4}{|l|}{ Category D: Financial Parameters } \\
\hline Return on debt (\%) & 3.7 & 3.7 & 5.6 \\
\hline Return on common stock (\%) & 6.5 & 6.5 & 9.75 \\
\hline Return on preferred stock (\%) & 4.5 & 4.5 & 6.75 \\
\hline Capital cost escalation $(\% / y r)$ & 0 & 0 & 2 \\
\hline Operating cost escalation $(\% / y r)$ & 0 & 0 & 2 \\
\hline Maintenance cost escalation $(\% / y r)$ & 0 & 0 & 2 \\
\hline Cover cost $\left(\$ / m^{2}\right)$ & 0 & 0 & 1.5 \\
\hline Liner cost $(\$ / h a)$ & 4000 & 5000 & 6000 \\
\hline
\end{tabular}


current knowledge of mass culture facilities, is a point of departure for further evaluation of cost improvements that could be achieved through continued research and technical progress.

\section{Specific Data}

Each parameter used in the algal production and economic model was assigned a reference value within a range of values based on information in the literature and the judgment of experts who reviewed concepts and offered direction in setting ranges.

In the sections below the input parameters used in the algal production and economic model are divided into four groups representing resource, biology, facility design, and financial information. The determination of the base value and range for each parameter is described for each general category. Table 3-1 summarizes the range of values for all parameters and lists the reference value for each.

\subsubsection{Resource Parameters}

\section{Evaporation}

Evaporation from a culture pond's surface is the major source of water loss from outdoor microalgal cultures. Gross evaporation is typically reported as "pan A" evaporation rate, while net evaporation rates include precipitation that offsets gross evaporation. Figure 3-2 illustrates average annual net "pan A" evaporation rates for the United States.

The pan A net evaporation data in Figure 3-2 must be modified to estimate evaporation rates for open reservoirs. Benemann (1982) reports that reservoir net evaporation rates are $50 \%-70 \%$ of pan A net evaporation rates, although the net evaporation in open culture microalgae systems would be higher than for open reservoirs because the algal ponds are shallower, absorb more light, and are stirred.

Vigon et al. (1982) reported that gross reservoir evaporation rates in the Southwest range from less than 1.00 to more than $2.00 \mathrm{~m} / \mathrm{yr}$ and selected an average precipitation rate of $0.2 \mathrm{~m} / \mathrm{yr}$ for their analysis of microalgae production, resuiting in net evaporation rates in the Southwest between 0.82 and $1.82 \mathrm{~m} / \mathrm{yr}$. These net reservoir evaporation rates are consistent with the $0.75-$ to $2.0-\mathrm{m} / \mathrm{yr}$ range indicated in Figure 3-2 for the desert Southwest.

For this analysis, we initially selected this range of $0.82-1.82 \mathrm{~m} / \mathrm{yr}(0.0022-0.005 \mathrm{~m} / \mathrm{d})$ to determine net evaporation in microalgae culture systems located in the Southwest. However, based on information from consultants, a value of $0.01 \mathrm{~m} / \mathrm{d}$ for the high end of the range was finally selected; this value reflects peak evaporation rates during part of the year. The range of $0.002-0.01 \mathrm{~m} / \mathrm{d}$ chosen for this analysis is comparable to "pan $\mathrm{A}^{\text {" }}$ data for the Southwest. Also, the value of $0.01 \mathrm{~m} / \mathrm{d}$ presents a very conservative (resource-intense) view of net evaporation in mass culture facilities. The microalgae reference system assumes a net evaporation rate of $0.0035 \mathrm{~m} / \mathrm{d}(1.28 \mathrm{~m} / \mathrm{yr})$.

\section{Salinity of Source Water}

The salinities investigated cover the range found in saline groundwaters of the southwestern United States (Feth et al. 1965). The salinity levels that distinguish potable from nonpotable water are not clearly defined. While U.S. Public Health Service standards specify not more than $0.5 \mathrm{~g}$ TDS/L in potable waters, waters with about 


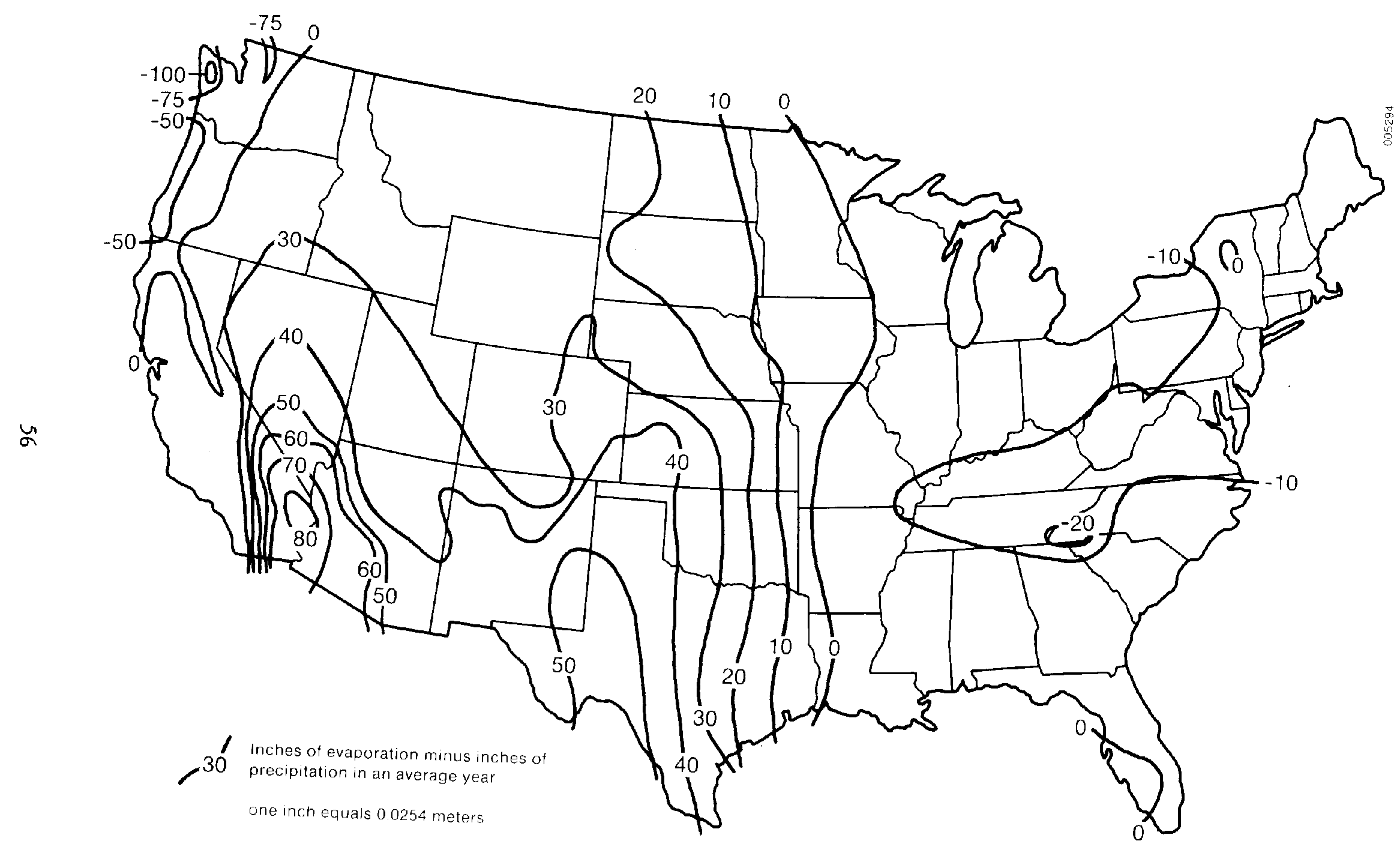

Figure 3-2. Average Annual Net Evaporation from Pan A Data 
$2 \mathrm{~g}$ TDS/L are used for domestic water supply in some regions. In addition, crops are being developed that will grow at salinities significantly higher than previously accepted for plants. Thus, $2.5 \mathrm{~g}$ TDS $/ \mathrm{L}$ was selected as the lower salinity limit for available groundwaters and $35 \mathrm{~g}$ TDS/L as the upper limit. Isolated wells have salinities greater than $25 \mathrm{~g}$. In contrast, the salinity of seawater is relatively constant at about $33 \mathrm{~g} \mathrm{TDS} / \mathrm{L}$. The reference value $25 \mathrm{~g}$ TDS/L, imposes on the system the use of highly saline water with little or no alternative uses.

\section{Energy Cost}

Electrical costs at large industrial facilities are composed of demand charges and energy charges. Demand charges are established in the utility rate base as the measured peak instantaneous power demand (in kilowatts) for the facility. Energy charges represent the actual consumption charged to an industrial facility.

The reference system assumes an electrical cost of $\$ 0.065 / \mathrm{kWh}$. Within the Southwest, comparable electrical energy costs (e.g., demand charges plus energy charges) from investor-owned utilities range from $\$ 0.04$ to $\$ 0.053 / \mathrm{kWh}$ (Edison Electric Institute 1982). A more conservative high end of this range was selected based on information from the Energy Information Agency (1983), which indicates that a few utilities in the Southwest may charge industrial users up to $\$ 0.10 / \mathrm{kWh}$. Therefore, depending on the utility company, the estimated cost of electrical energy for a microalgae facility ranges from $\$ 0.040$ to $\$ 0.10 / \mathrm{kWh}$. The reference value of $\$ 0.065 / \mathrm{kWh}$ is about the midpoint of that range.

\section{$\underline{\text { Land Costs }}$}

A common assumption associated with the microalgal fuel production concept is that low-value, arid land deemed unsuitable for other uses will be used for mass culture facilities. Benemann et al. (1982) estimated that land costs between $\$ 1185$ and $\$ 1480 /$ ha can be anticipated in such areas. In comparison, irrigated cropland could cost over $\$ 7200 /$ ha. For the reference system land costs were $\$ 1245 /$ ha. And the low and high values for sensitivity analyses are $\$ 1000$ and $\$ 1500 /$ ha, respectively.

\section{Nutrient Costs}

The major nutrients required for mass cultivation of microalgae for fuel are carbon, nitrogen, phosphorus, and potassium. Each nutrient can be supplied in various watersoluble forms that can be directly utilized by the microalgae.

Carbon cost estimates are based on the use of carbon dioxide obtained from flue gas. While certain levels of bicarbonate are available in the saline source water, additional carbon is required to support the high productivity of microalgae. This additional carbon from flue gas was costed based on information from the petroleum industry, which uses large quantities of flue gas carbon dioxide in enhanced oil recovery projects (Anada et al. 1983). Because the price of carbon dioxide from a flue gas source depends on the quantities of carbon dioxide required and the distance transported, the microalgae production and economic model used in this analysis determines the quantity of carbon dioxide required to support the assumed productivity level and then, based on transport distance, determines the price of delivered carbon dioxide to the facility.

The model $\mathrm{CO}_{2}$ costs are calibrated to the cost of flue gas from a coal-fired power plant. The carbon dioxide must be scrubbed from the flue gas using a potassium carbonate 
recovery system, dried, and compressed for economical transport via pipeline. The pipeline size and required distance represent additional costs for the delivered carbon dioxide.

The reference case assumes a pipeline distance of $80 \mathrm{~km}$. Carbon dioxide can be transported as far as $300 \mathrm{~km}$. Based on reference system productivity levels of $17 \mathrm{~g} / \mathrm{m}^{2} / \mathrm{d}$ and the availability of $200 \mathrm{~g}$ carbon $/ \mathrm{m}^{3}$ in the source water, the reference system microalgae facility requires $83 \times 10^{3} \mathrm{~m} / \mathrm{d}$. The delivered price for this carbon dioxide is $\$ 0.13 / 10^{3} \mathrm{~m}^{3}$.

Nitrogen is supplied as ammonia gas at a cost of $\$ 185 / \mathrm{t}$. Potassium is supplied as potassium muriate at a delivered price of $\$ 92 / t$, and phosphorus is supplied as superphosphate at a delivered price of $\$ 254 / \mathrm{t}$. The amount of nutrients required is determined by productivity levels within the culture system; like carbon, these nutrients may be partially supplied by the source water.

Nutrient prices are based on supplier quotes for spot purchases as listed in the Chemical Marketing Reporter (1984) and do not reflect contract prices that might be available to users of large quantities of industrial chemicals. Contract prices for the chemicals used in microalgae production may be lower than these spot prices; alternatively, actual delivered prices for these nutrients may be higher due to delivery charges to remote locations in the Southwest.

The reference system values for delivered prices of these noncarbon nutrients reflect spot prices for ammonia gas, potassium muriate, and superphosphate. The range for each nutrient is about $10 \%$ above and below the spot price to account for the uncertainty in actual delivered prices.

\section{Nutrients in Source Water}

Reference system values for concentrations of nitrogen, carbon, and potassium in the source water are drawn from data on saline well water presented by Feth et al. (1965), and from the results of a multivariate statistical analysis of New Mexico groundwater (Barclay 1984a). Neither study includes data for phosphorus.

Feth et al. (1965) present nitrogen (as nitrate) content data for wells in New Mexico. The mean nitrate content of all well waters with salinities greater than $4 \mathrm{~g}$ TDS/L was $13 \mathrm{~g} / \mathrm{m}^{3}$, which was taken as the reference value. Some well waters contained no detectable nitrate, whide the maximum reported by Feth was $96 \mathrm{~g} / \mathrm{m}^{3}$. Well logs have shown that a few wells have nitrate concentrations in excess of $10,000 \mathrm{~g} / \mathrm{m}^{3}$, but these data appear to be anomalous, and sustainability of such a supply would have to be questioned (Barclay 1984b). If such concentrations were sustainable, these wells would represent a significant nutrient source. Unpolluted seawater can contain up to $0.6 \mathrm{~g} \mathrm{~N} / \mathrm{m}^{3}$ when collected from deep ocean sources.

The reference phosphorus or phosphate content of the source water $\left(0.5 \mathrm{~g} / \mathrm{m}^{3}\right)$ was estimated by assuming a nitrogen:phosphorus ratio of approximately 30 for the reference case; a range of $\pm 100 \%$ was arbitrarily established for phosphorus content. The phosphorus content of unpolluted seawater seldom exceeds $0.1 \mathrm{~g} / \mathrm{m}^{3}$.

The reference value for potassium $\left(46 \mathrm{~g} / \mathrm{m}^{3}\right)$ is the same as Barclay's Type I low-salinity water, which is an average associated with water that has a salinity of $8 \mathrm{~g}$ TDS/L, the 
reference value (Barclay 1984a). Data presented by Feth et al. (1965) for Arizona and New Mexico showed potassium concentrations between 7.6 and $372 \mathrm{~g} / \mathrm{m}^{3}$ for saline

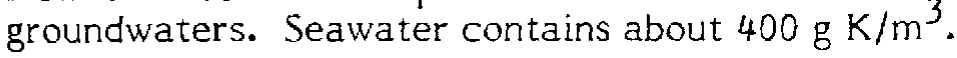

Many groundwaters contain significant concentrations of bicarbonate ions, in sharp contrast to seawater, which contains only about $25 \mathrm{~g} / \mathrm{m}^{3}$ of total dissolved carbon dioxide (dissolved $\mathrm{CO}_{2}$ plus bicarbonate plus carbonate). Barclay's Type I low-salinity water contains about $500 \mathrm{mg} \mathrm{HCO}_{3}{ }^{-}$, which is equivalent to about $100 \mathrm{~g} / \mathrm{m}^{3}$, the value chosen for the reference case (Barclay 1984a). Feth's data show bicarbonate concentrations as low as $50 \mathrm{~g} / \mathrm{m}^{3}$, while Barclay shows concentrations of bicarbonate up to $2100 \mathrm{~g} / \mathrm{m}^{3}$. These values are equivalent to the minimum and maximum, values of source water inorganic carbon content chosen for the analysis $\left(10\right.$ and $\left.420 \mathrm{~g} / \mathrm{m}^{3}\right)$.

\subsubsection{Facility Design Parameters}

\section{Effective Culture Area}

The effective culture area parameter apportions the total facility size to land area under cultivation and land area used for "nonproductive" accoutrements such as harvesting systems, buildings and laboratories, access roads, pipe runs, and the headbox. Based on preliminary design analysis, the effective culture area was found to be relatively insensitive to module size, ranging from $85.6 \%$ for 20 -ha ponds to $86 \%$ for 60 -ha ponds.

The reference case assumes an effective culture area ratio of $86.0 \%$. Because of uncertainty in this value until more extensive research is conducted, the sensitivity range for this parameter is from $70.0 \%$ to $90.0 \%$.

\section{Downtime}

Additional cultivation surface area is lost due to downtime. This parameter reduces the total production surface area due to cleaning, repairing, draining, and filling ponds. The reference case assumes a downtime of $10 \%$. The low value in this sensitivity range was arbitrarily set at $30 \%$ (most downtime) and the high value at $5 \%$ (least downtime).

\section{Module Size}

Module size is another parameter for which little information is available. The available data indicate that certain economies of scale may be achieved by increasing the size of a module or pond, although there is likely to be a loss of operational control with increasing size. Benemann et al. (1982) presented a cost analysis of microalgae cultivation based on a facility composed of twenty 40 -ha ponds.

The reference system assumes a similar size module or pond. Thus, the reference system is composed of forty-three 20-ha modules that have a total surface area of 860 ha (86\% of nominal facility size), of which 774 ha are under productive cultivation at any given time ( $10 \%$ reduction for downtime). The reference size of the individual modules (20 ha) represents a significant increase in scale over current technology. For this reason, 10 ha was selected as the lower value of module size for the sensitivity analysis, and 40 ha was selected as the upper value. 
Channel Width

Within each module the reference design assumes that the total surface area is divided into individual channels. The maximum width of a channel is determined by the largest paddlewheel capable of operating in the channel. Benemann et al. (1982) reported that the largest conceivable paddlewheel is approximately $60 \mathrm{~m}$; this value was selected as the maximum channel width.

As before, the uncertainty in constructing and operating a paddlewheel mixing system of this size necessitated sensitivity ranges for channel width that were determined according to input from consultants. While a $60-\mathrm{m}$ width was deemed possible with careful engineering, a channel width of $6 \mathrm{~m}$ was also examined to estimate the impact on costs of employing narrower channels. The reference system assumes a channel width of $30 \mathrm{~m}$.

\section{Channel Roughness}

Channel roughness is used to calculate head loss and thus to estimate power requirements to circulate the culture medium within a module. In shallow, open-channel systems, headloss is calculated from the Manning's equation, which requires a channel roughness parameter "n." Average values for n, obtained from Perry and Green (1984) indicate ranges from 0.023 for earthen channels that are straight and uniform to 0.03 for winding, sluggish channels with pools and eddies. Based on this parameter range, the reference roughness value is assumed to be 0.025 , with variation in this value consistent with the above range.

\section{Mixing Velocity}

The speed at which the culture medium is circulated within the module is a trade-off between keeping the algae in suspension and increased power requirements. Power requirements increase as the cube of the velocity; however, increased velocity induces more turbulence in the culture system, which will provide for better culture mixing and may lead to higher productivity.

The reference system's nominal mixing velocity is $0.2 \mathrm{~m} / \mathrm{s}$ based on similar mixing speeds used in experimental cultivation facilities. To determine the cost sensitivity of the mixing speed, the low value of mixing speed employed was $0.05 \mathrm{~m} / \mathrm{s}$ and the high value $0.3 \mathrm{~m} / \mathrm{s}$.

\section{Culture Depth}

Culture depth in SERI-sponsored experimental microalgae cultivating facilities has varied from 0.1 to $0.3 \mathrm{~m}$. Experience has demonstrated trade-offs in culture depth; shallower systems support greater culture densities and result in lower harvest costs than deeper systems. But shallower systems require higher mixing speeds and thus higher energy costs. Deeper systems provide greater temperature stability. The reference culture depth is $0.15 \mathrm{~m}$ and a range of $0.1-0.3 \mathrm{~m}$ is considered.

\section{Nutrient Concentrations in Medium}

Concentrations of nutrients in the culture medium determine the quantity of nutrients lost with the water that enters the product stream and exits in the blowdown stream. The availability of nutrients to the algae is also determined by the concentration in the 
medium, but algae can obtain nutrients from very low medium concentrations. The major difficulty in maintaining low-standing nutrient concentrations lies in the development of a control system that can supply nutrients at the same rate at which they are required by the algae for growth. Historically, this problem has been solved by providing the aigae with high standing nutrient concentrations to eliminate the risk of nutrient shortage, and accepting the associated costs of nutrient loss.

The reference value for carbon content (total $\left.\mathrm{CO}_{2}\right)$ of the medium $\left(12 \mathrm{~g} / \mathrm{m}^{3}\right)$ has been observed to support the growth of Phaeodactylum tricornutum (Caperon and Smith 1978). The maximum value $\left(25 \mathrm{~g} / \mathrm{m}^{3}\right)$ represents the approximate total $\mathrm{CO}_{2}$ content of seawater, while the minimum value $\left(7.5 \mathrm{~g} / \mathrm{m}^{3}\right)$ represents a value at which the growth of $P$. tricornutum is moderately carbon-limited.

The dependence of the phytoplankton growth rate on medium concentrations of various nitrogen sources has been extensively investigated (e.g., Caperon and Meyer 1972). Normally, algae can grow at concentrations of inorganic nitrogen as nitrate or ammonium that are nearly undetectable by present techniques--less than about 1 micromolar. Growth occurs near the maximum rate at concentrations of about 10 micromolar. Nevertheless, nutrient concentrations in outdoor cultures have frequently been maintained in the range of 100-1000 micromolar for the reasons discussed above. The reference case values for medium content of nitrogenous nutrients is $0.28 \mathrm{~g} / \mathrm{m}^{3}$, while the minimum value is 10 micromolar $\left(0.14 \mathrm{~g} / \mathrm{m}^{3}\right)$ and the maximum is 1000 micromolar $\left(14 \mathrm{~g} / \mathrm{m}^{3}\right)$.

Microalgal growth is sustained at concentrations of phosphate that are as low as or lower than those observed for nitrogen sources (e.g., Terry 1982). Thus, the molar values selected for the medium phosphate concentrations were identical to those selected for medium nitrogen concentrations. These molar values for phosphate are $0.31,0.62$, and $31 \mathrm{~g} / \mathrm{m}^{3}$.

Few data are available on the growth response of microalgae to potassium concentrations. Input from the microalgae assessment consultant team indicated that at least some species can grow rapidly at very low potassium concentrations, and $5 \mathrm{~g} / \mathrm{m}^{3}$ was chosen as a minimum. McLachlan (1964) suggests that the response to potassium concentrations in the medium is species specific; some species are capable of growth at these low concegtrations. The reference value is set at $25 \mathrm{~g} \mathrm{~K} / \mathrm{m}^{3}$ and the upper bound at $400 \mathrm{~g} \mathrm{~K} / \mathrm{m}^{3}$.

\section{Carbon and Nitrogen Losses}

If the carbon dioxide content of the nutrient medium exceeds the value expected in equilibrium with the atmosphere, significant losses of $\mathrm{CO}_{2}$ can occur through the culture surface. Control of such losses will be a critical feature of the carbon dioxide system. With proper control, it is likely that these losses can be completely eliminated. The minimum value in the sensitivity analysis represents no losses, while the reference value represents an equilibrium exchange between the culture surface and the atmosphere. The maximum value is an arbitrarily extreme rate $\left(3.0 \mathrm{~g} / \mathrm{m}^{2} / \mathrm{d}\right)$. A similar logic was used to develop nitrogen losses from the culture surface.

\section{Mixing System Efficiency}

Recirculation within a culture system may be accomplished by a variety of mechanical devices. Because shear stresses within many pumping devices may break algal cell walls, 
the various options typically considered by aquaculture operators include paddlewheels and air lift pumps. A major distinction between these two mixing systems is the efficiency of the pump. Paddlewheel efficiencies are about 60\% (Benemann et al. 1982), whereas air lift pump efficiencies are about $30 \%$. Additional efficiency losses of up to $24 \%$ are experienced in the drive system.

The reference case assumes a mixing efficiency characteristic of a paddlewheel system (60\%). To evaluate the cost sensitivity to mixing system selection, the sensitivity analysis examines trade-offs between an air lift pump mixing system (e.g., mixing pump efficiency of $30 \%$ ) and an improved mixing system design having pump efficiency of $75 \%$.

\section{Harvester Solids Removal Efficiency}

The reference case assumes a solids removal efficiency of $90 \%$. This relatively high efficiency level either presumes chemical additions similar to those described above or assumes that microalgae species of sufficient size are cultivated to obtain easy harvestability using nonchemical, conventional harvesting technology. Because of these assumptions, the sensitivity of solids removal efficiency allows for removal efficiencies as low as $50 \%$ and as high as $98 \%$.

\section{Harvester Subsystem}

The selection of a harvester subsystem for a mass culture system depends on a number of variables such as cell size, capital and operating costs, throughput, and efficiency. Many of these issues are discussed in earlier sections of this report.

The reference case was developed to incorporate a two-stage harvester system. The first stage is a microstrainer capable of handling large influent flow rates and achieving concentration ratios up to 10 . The second stage is a centrifuge system used to bring the product stream from the first stage up to a design criterion of $10 \%$ solids, which is suitable for subsequent processing and conversion to a fuel product. As an alternative firststage harvester system, the sensitivity analysis examines the use of continuous belt filter harvester systems with a concentration ratio of 70 , which requires a $50 \%$ higher capital investment.

\subsubsection{Biological Parameters}

\section{Operating Salinity Tolerance}

The salinity parameter employed here represents the expected salinity of the system under operating conditions, although the system salinity will drop temporarily after major rainstorms. Some species of algae will grow only in fresh water, while other species will tolerate saturated sodium chloride brines. Theoretically, algal systems can be operated anywhere in this range. Very high salinities, however, will present significant problems in equipment maintenance because of the extremely corrosive nature of the water. The lower limit of system operating salinity is established by the salinity of the source water employed. In all cases the operating salinity will be higher than that of the source water due to evaporation; the minimum operating salinity of $10 \mathrm{~g}$ TDS/L was chosen to be slightly higher than the reference case source water salinity of $8 \mathrm{~g} T D S / L$. The reference case salinity, $35 \mathrm{~g} \mathrm{TDS} / \mathrm{L}$, is close to that of seawater and is a salinity at which a number of mass culture systems have operated. A maximum salinity of $120 \mathrm{~g}$ TDS/L has been employed in systems for the culture of the halotolerant chlorophyte Dunaliella, and serves as the upper range value. 


\section{Phosphorus Content in Cells}

The phosphorus content in microalgal cells varies widely. While the algal requirement for phosphorus is not highly variable between species, there is considerable variability in the capacity of cells to store phosphorus. In many cases, this capacity for phosphorus storage can be extremely large. Thus, microalgal systems that are operated at saturated medium concentrations of phosphate produce cells heavily loaded with phosphorus. The values selected for the cell phosphorus content represent the phosohorus content of Phaeodactylum tricornutum under extreme nutrient limitation $(0.0012 \mathrm{~g} / \mathrm{g}$ dry weight), at optimum phosphorus content for total biomass production $(0.007 \mathrm{~g} / \mathrm{g}$ dry weight), and at phosphorus-saturated conditions $(0.023 \mathrm{~g} / \mathrm{g}$ dry weight).

\section{Productivity}

The productivity of the system is determined by three input parameters: depth, density, and detention time. These parameters are discussed in detail elsewhere in this report, but their selection was based on a range of values established for the potential productivity of microalgal systems. Productivities range from $19 \mathrm{~g}$ dry wt $/ \mathrm{m}^{2} / \mathrm{d}$ minimum value) to $25 \mathrm{~g} \mathrm{dry} \mathrm{wt} / \mathrm{m}^{2} / \mathrm{d}$ (reference value) to $60 \mathrm{~g}$ dry wt $/ \mathrm{m}^{2} / \mathrm{d}$ (maximum value). The reference value is representative of the rates that have been achieved in outdoor systems of conventional design. Rates near the minimum have been observed under less favorable conditions. The maximum value is representative of the rates achieved under carefully controlled conditions in outdoor raceway systems, where considerable energy is expended to enhance production. In subsequent analyses (Section 6.0 ), we treat these variables as design targets set to achieve system cost-effectiveness.

\section{Proximate Chemistry}

Proximate analysis of cellular biochemical composition calculates cell content of lipids, carbohydrates, proteins, and metabolic intermediates. The term "metabolic intermediate" refers to any component of the cell biomass not extracted in one of the other three classes. Since each component is presented as a fraction of the total cell biomass, the sum of these fractions must equal $100 \%$. Thus, the model accepts values for the content of lipids, carbohydrates, and intermediates (including ash); the remainder of the cell biomass is allocated to protein. The model flags the results of any calculation that leads to unrealistically low cell protein content. Individual members of this set of parameters can not be varied independently since increases in the content of one biochemical fraction must be accompanied by decreases in the content of the other fractions. Thus, these parameters were linked in the sensitivity analysis, and the three sets of parameter values were established to represent low, moderate, and high lipid concentrations. The low lipid content was taken from data collected by Laws (1984) for Platymonas sp. grown in outdoor cultures under nutrient-saturated conditions. This species, which does not produce lipid as a storage product, contained $20 \%$ lipid, $49 \%$ carbohydrate, and an estimated $10 \%$ metabolic intermediates. The high lipid content case represents the composition of the lipid-producing species Phaeodactylum tricornutum, grown under nitrogen-limited conditions (Terry et al. 1985). This species produced about $60 \%$ lipid, $10 \%$ carbohydrate, and $10 \%$ intermediates. The moderate lipid content (i.e., reference case) species are assumed to produce $30 \%$ lipid, $20 \%$ carbohydrate, and $10 \%$ metabolic intermediates, with an ash content of $8 \%$ and a nitrogen content of $32 \%$. 


\subsubsection{Financial Parameters}

\section{Escalation Rate}

The analysis presented is this report uses constant 1984 dollars for reporting costs to avoid an additional assumption regarding future inflation rates. The reference escalation rates are based on zero real increases in the costs of goods and services. Because of the uncertainty in future projections of real escalation rates, the sensitivity analysis examined a $2 \%$ rate of escalation for all cost categories.

\section{Capital Investment Leveraging}

Most capital investment projects involve a combination of equity capital and borrowed money. Leveraging projects can provide a means for altering the rate of return for investors. If the after-tax cost of borrowed money is less than the discounted rate of return, it is economically desirable to borrow money rather than investing equity capital into a project.

The reference case presumes a project financing scheme in which $30 \%$ of the investment is borrowed and the balance is financed through stock equity. Another consideration in estimating the costs of financing a mass culture facility is the required rate of return for the debt portion and equity portion. The reference returns are $3.7 \%, 6.5 \%$, and $4.5 \%$ on debt, common, and preferred stock, respectively. These reference rates are increased by $50 \%$ to represent a high cost of capital case but are held constant at the reference level in the low case sensitivities.

\section{Capital Cost}

While considerable attention was devoted to developing realistic cost estimates for each component of the mass culture facility, questions on the validity of the capital cost estimates will remain until a more detailed analysis can be completed. Short of conducting such analysis, the sensitivity cases to evaluate possible changes in capital investment requirements for the mass culture project were based on $\pm 25 \%$ changes in total capital investment from the reference case. This can be interpreted as representing a case where capital costs for the balance of the system are not precisely identified in the reference systems due to the rudimentary status of development of the technology.

\subsubsection{Summary}

Parameter value ranges and reference case values are summarized in Table 3-1 for the four groups of parameters.

\subsection{Reference Case Economics}

The economics of the reference system are developed by two models: the microalgae production model and a revenue requirement economic model. The microalgae production model develops first-year cost estimates for a facility and determines production levels based on user-defined inputs and requirements. These first-year costs and production levels are then transferred to the revenue requirement model, which determines the required selling price of the algal products to achieve a specified minimum return on 
equity investment. These prices are determined by annualizing the present value of all facility cost streams and dividing these annual costs by the production volume. All costs are reported in constant $1984 \$$.

The total estimated present value capital investment for the microalgae reference system is $\$ 37$ million, which represents the installed depreciable and nondepreciable costs for the entire 1000-ha facility ( 860 ha are actually available for cultivation). The unit cost for the facility is $\$ 43,283 / \mathrm{t}$ a or $\$ 4.33 / \mathrm{m}^{2}$ of cultivation surface area or $\$ 4.33 / \mathrm{m}^{2}$.

Total annual operating and maintenance costs are $\$ 8.2$ million. These direct production costs, at $5 \%$ PSE or $17 \mathrm{~g} / \mathrm{m}^{2} / \mathrm{d}$, equal $\$ 393 / \mathrm{t}$ algae produced.

By annualizing the capital investment over the facility's lifetime, the total annualized production cost is about $\$ 13$ million/yr. Total algal biomass production costs are $\$ 393 / t$ or $\$ 0.39 / \mathrm{kg}$ based on a net algal biomass yield of 33,171 t/yr. At a $30 \%$ lipid content, the total annual lipid yield for the reference system facility is $71,012 \mathrm{bbl}$.

\subsubsection{Cost Contribution to Reference Case}

One method for evaluating the potential for reducing costs through continued research is an examination of the individual cost centers to determine which ones contribute significantly to the final product cost. These cost centers can be grouped in separate categories such as exogenous costs and endogenous costs to indicate that the cost is not or is, respectively, affected as a result of program $R \& D$. Additional detail within each of these general categories can provide specific information regarding the relative importance of specific subcategories to final product cost.

The general cost contribution to the microalgae reference case is illustrated in Figure 3-3. Operating costs represent about $68 \%$ of the total product cost. Capital costs, including both depreciable and nondepreciable capital investments, represent $23 \%$ of the total product cost and maintenance costs contribute almost $9 \%$ to the total annual cost of production.

\section{Contribution of Direct Costs}

Direct production costs, or the combined annual maintenance and operating costs, represent the largest cost category. These combined costs contribute $76.7 \%$ of the total production cost of microalgae lipids, providing a cost contribution of $\$ 302 / \mathrm{t}$ of algae produced. Because these direct costs are so significant, it is worthwhile to examine the subcategories within this general cost category to identify the major cost centers.

Figure 3-4 illustrates the subcategories for the direct cost of maintenance and operation. As a percentage of total present value, nutrient expenses are $33.7 \%$ and are the largest cost category within the direct expenses. These nutrient expenses include carbon, nitrogen, and phosphorus. Potassium expenses are zero in the reference system because the saline source water is assumed to contain sufficient quantities of potassium to support a productivity level of $17 \mathrm{~g} / \mathrm{m}^{2} / \mathrm{d}$. Carbon dioxide expenses represent $78.6 \%$ of the total nutrient expenses, or about $26 \%$ of total cost.

Labor and overhead are the second largest cost category, contributing about $24 \%$ to total production cost. Water, the third largest portion of cost (almost 16\%), is a vital component of production and is subject to considerable uncertainty given the range of 


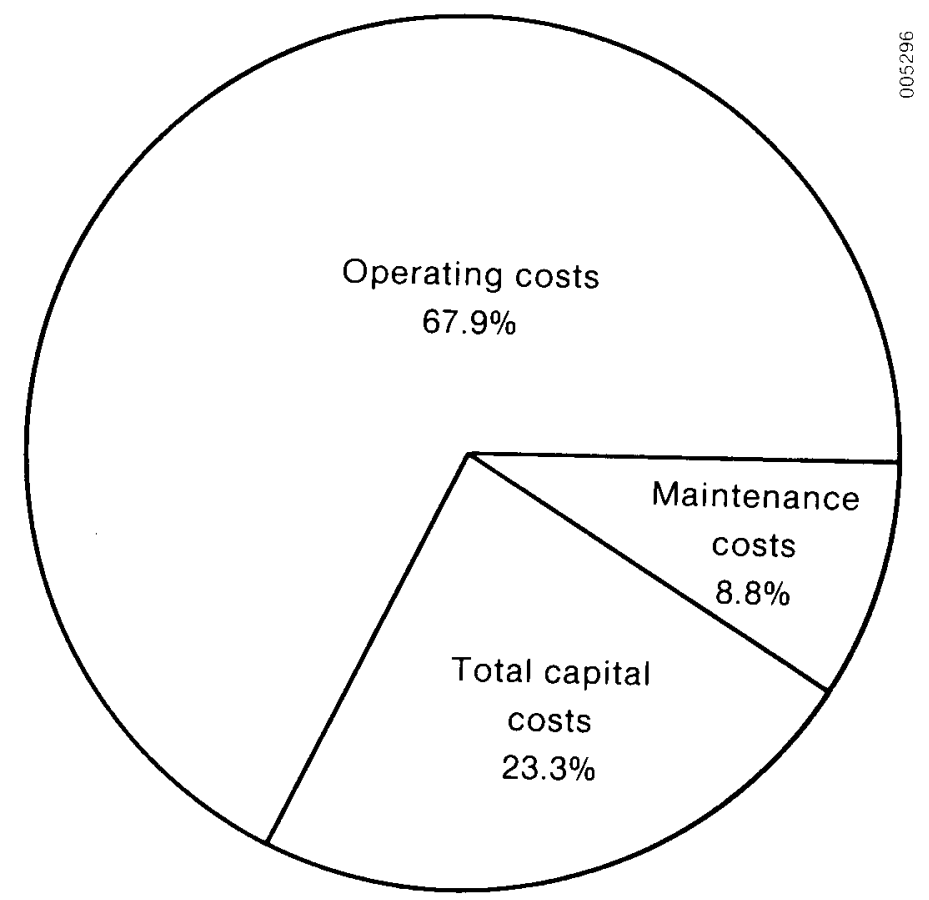

Figure 3-3. Contribution to Product Cost by General Cost Category

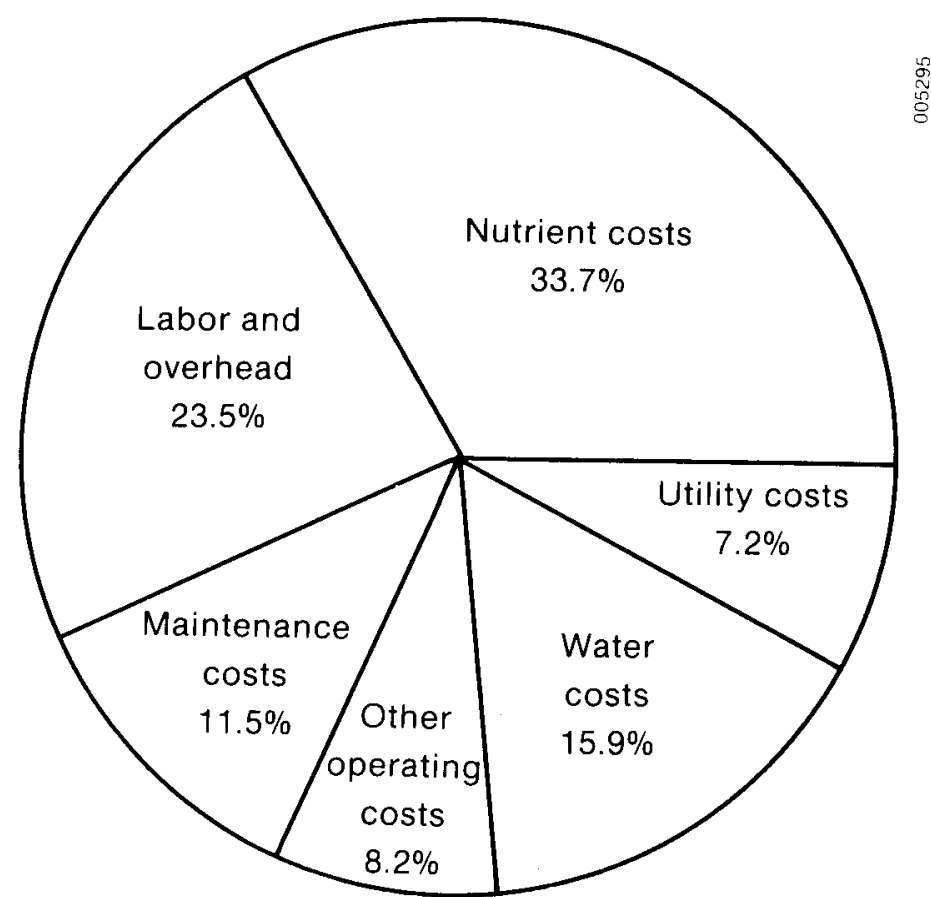

Figure 3-4. Cost Contribution Detail for Direct Operating and Maintenance Expenses 
possible costs discussed above. The other major factor input, electricity, contributes a substantial $7 \%$ to costs and, like water, is an area of considerable uncertainty. Other operating costs, estimated as $7 \%$ (nominal) of other operating costs, provide for a general category of maintenance expenses such as liner repair.

\section{Contribution of Capital Costs}

The total capital costs for the reference facility are about $\$ 37$ million and are the fixed costs incurred with design and construction of the mass culture facility. The fixed costs shown in Figure 3-3 represent $23.3 \%$ of the total product costs. Within this general cost category, depreciable equipment costs are $51 \%$ of the total present value for capital.

Figure 3-5 presents a summary of the relative contribution of the major capital cost components, combining both depreciable and nondepreciable investment categories. The culture system was estimated to contribute $37.7 \%$ of the total capital investment. The costs in this category include module construction, internal distribution systems for nutrients and water, pond lining, mixing systems, buildings, and electrical service to the facility site. Site preparation and surveying account for $26.2 \%$ of capital costs and the harvester subsystems represent $13 \%$ of the total capital investment. Because of harvester design capacity limitations, the reference system requires 22 microstrainers that have a design capacity of $13.3 \mathrm{~m}^{3} / \mathrm{min}$ per machine and eight second-stage centrifuges to concentrate the microalgae to at least $10 \%$ solids.

\section{Nondepreciable Capital Investment}

Nondepreciable capital investment items include site preparation and surveys, land costs, engineering fees, and contingency allowances; these items are nonequipment expenditures that cannot be depreciated for tax purposes. Together, these cost categories represent $49 \%$ of the total present value of the capital investment. This relatively high percentage is due to the site preparation and survey costs, which are major cost items in pond construction. The distribution of these nondepreciable capital items is presented in Figure 3-6.

Contingency allowances for new construction typically are between $10 \%$ and $15 \%$, depending on the stage of development of the technology and whether prior construction experience has been obtained. The novel concept of mass culturing microalgae for fuel requires a higher contingency fee of $20 \%$ (nominal) of total capital investment plus engineering fees, which results in relatively high costs; however, as construction experience is gained with these systems, these costs can be expected to decrease (Huguenin 1984).

Engineering and architectural fees for new construction range between $4.9 \%$ and $16 \%$ of project cost. Nominal fees for this category were based on several points. While most of the construction expense is for earthmoving, the scale of the facility may present unique engineering problems. Thus, a $15 \%$ fee for mechanical equipment and an $8 \%$ fee for earthwork were selected as nominal values for the reference case. The contribution of these engineering fees to total nondepreciable capital investment is $15.5 \%$ (Huguenin 1984).

Land costs represent $7.8 \%$ of the nondepreciable capital investment. This low percentage is expected because of the low value of the marginal land contemplated for mass culturing microalgae. 


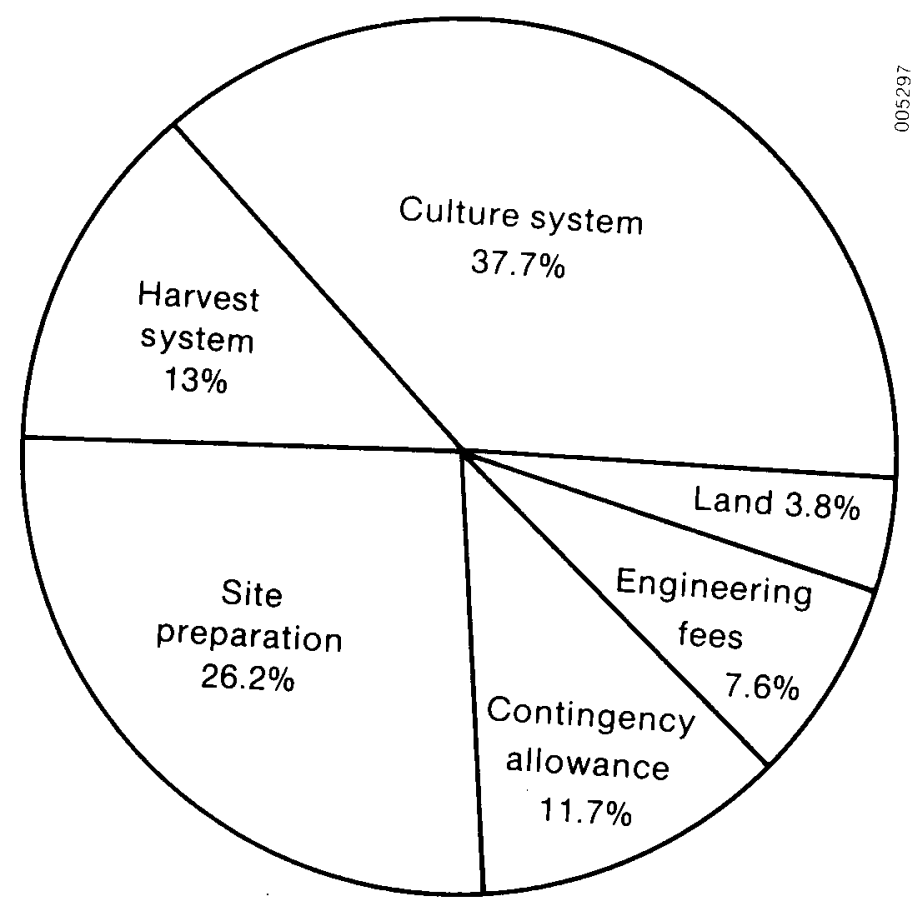

Figure 3-5. General Cost Contribution of Capital Cost Categories

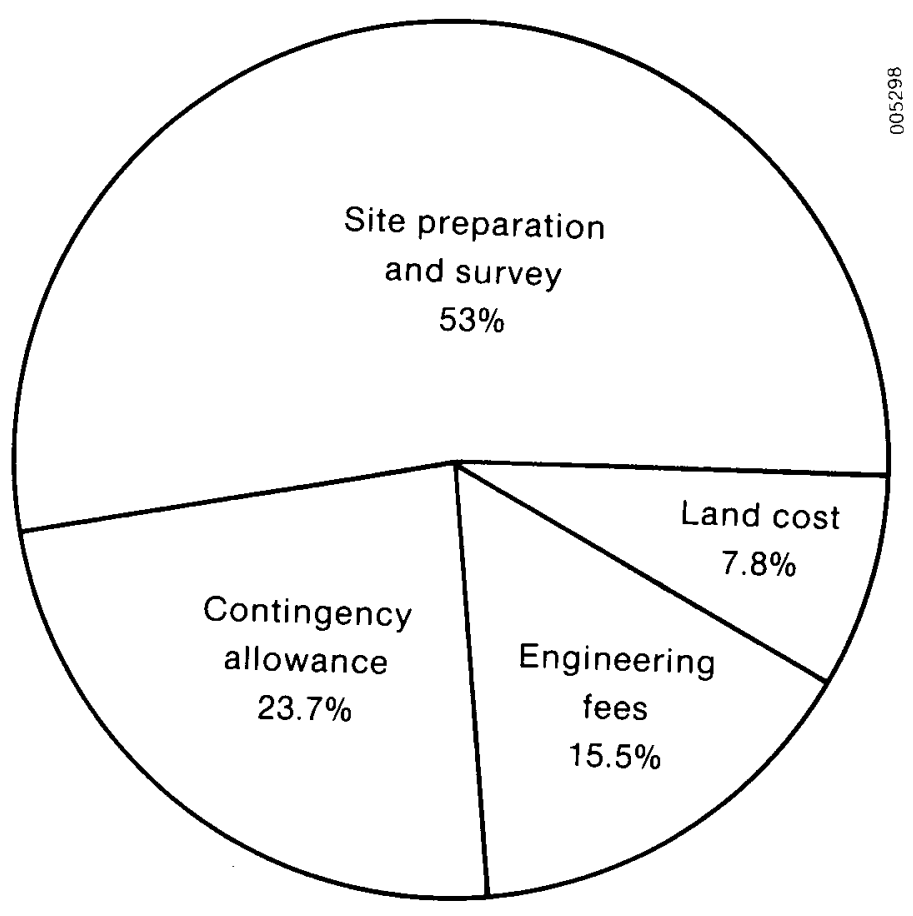

Figure 3-6. Cost Contribution Detail for Nondepreciable Portion of Capital Investment 


\section{Depreciable Capital Investment}

Depreciable capital investment includes equipment items that may be depreciated over a period of time established by the IRS. Specific lifetimes differ for various pieces of equipment, and while the analysis does not account for different lifetimes, a maintenance operating cost has been included as a sinking fund for equipment replacement. Also, by extending the depreciable lifetime of all equipment to 15 years, product costs increase because faster depreciation schedules allow quicker write-offs, thus decreasing revenue requirements.

Of the total depreciable investment, the largest contributing cost category was the firststage harvesting system. As illustrated in Figure 3-7, almost $26 \%$ of the total equipment investment is to purchase and install the microstrainers and centrifuges necessary for harvesting. Lining the pond bottoms contributes $26 \%$ to capital equipment costs. Other major equipment items include construction of the pond dike and piping system (14\%), the paddlewheel mixing system (13.1\%), and the electrical system to service the facility (9.1\%).

\subsubsection{Summary}

Table 3-2 presents a summary of the various cost contributions to reference case product costs for each general and specific cost category. The final cost estimate for producing microalgae in the reference case is $\$ 393 / t(1984 \$)$. For a research program with the goal of producing cost-competitive liquid fuels early in the next century, the present economics for mass cultivation are unfavorable; however, the production cost estimates in Table 3-2 reflect an opinion of what could be accomplished using the present capability of mass culture techniques to produce liquid fuels. These results presume one set of parameters derived by consensus; however, other plausible conditions could occur. Section 5.0 examines the cost sensitivity of these reference system parameters to variation within defined limits. First, we examine concepts for refining the raw algal product into useful fuels that compete with conventional, hydrocarbon-based products.

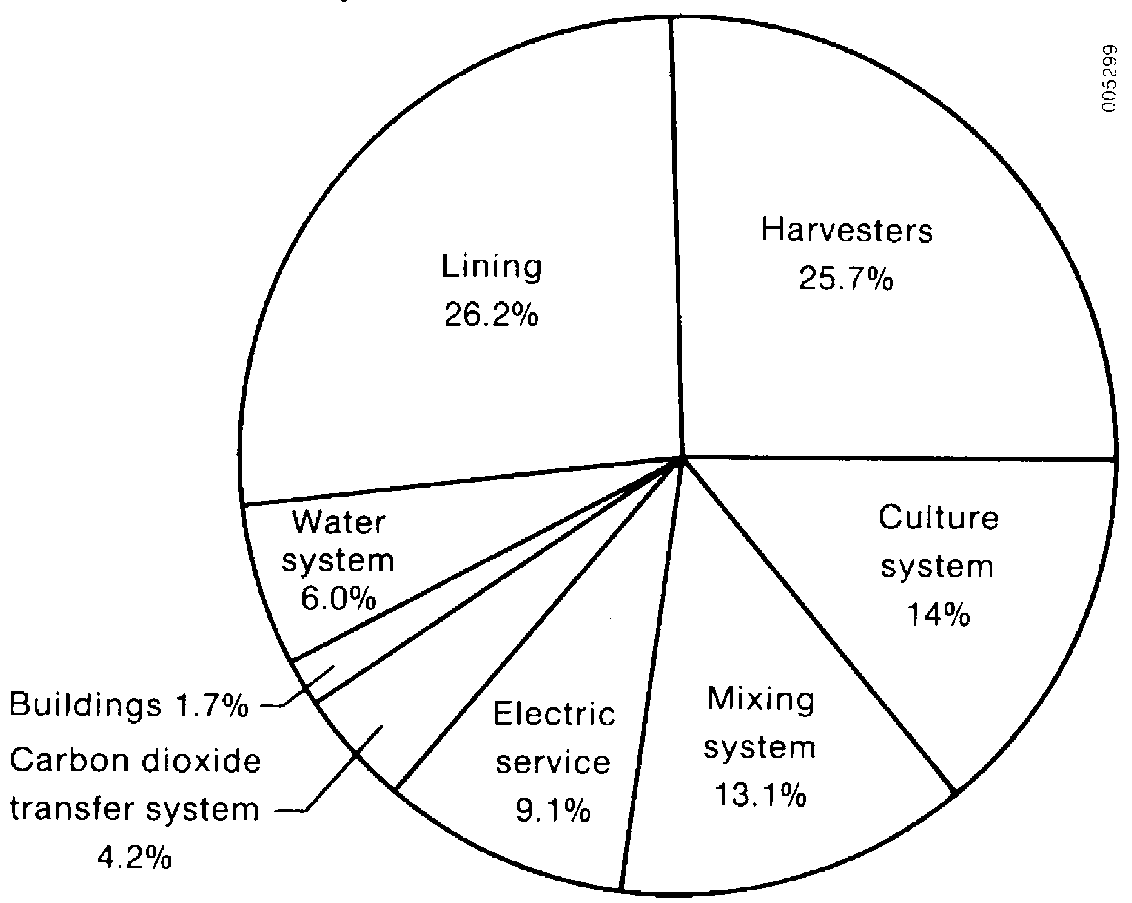

Figure 3-7. Cost Contribution Detail for Depreciable Capital Investment 
Table 3-2. Summary of Reference Production Facility Cost Contributions for Annual Direct Cost and Capital Cost (1984\$)

\begin{tabular}{|c|c|c|}
\hline Cost Category & $10^{6} 1984 \$ / y r$ & $\$ / t^{a}$ \\
\hline \multicolumn{3}{|l|}{ Capital Costs } \\
\hline $\begin{array}{l}\text { Site preparation } \\
\text { Culture system } \\
\text { Harvester systems } \\
\text { Engineering fees } \\
\text { Contingency } \\
\text { Land }\end{array}$ & $\begin{array}{l}0.796 \\
1.146 \\
0.395 \\
0.231 \\
0.356 \\
0.116 \\
\end{array}$ & $\begin{array}{r}24 \\
35 \\
12 \\
7 \\
11 \\
3 \\
\end{array}$ \\
\hline Total capital cost & 3.040 & 92 \\
\hline \multicolumn{3}{|l|}{ Operating Costs } \\
\hline $\begin{array}{l}\text { Labor and overhead } \\
\text { Utility } \\
\text { Nutrients } \\
\text { Water } \\
\text { Operations } \\
\text { Maintenance }\end{array}$ & $\begin{array}{l}2.354 \\
0.713 \\
3.374 \\
1.588 \\
0.822 \\
1.151 \\
\end{array}$ & $\begin{array}{r}70 \\
21 \\
102 \\
48 \\
25 \\
35 \\
\end{array}$ \\
\hline Total operating cost & 10.002 & 301 \\
\hline Total feedstock cost & 13.042 & 393 \\
\hline
\end{tabular}

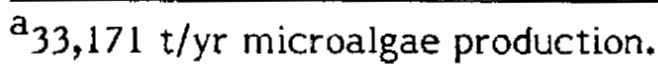




\subsection{ECONOMICS OF PRODUCING FUELS FROM MICROALGAE}

In the previous section we discussed the economics of mass microalgae culture as they relate to applications in the southwestern United States. We now turn to a discussion of the final component of the concept under review, the economics of producing fuels from microalgal feedstocks. The main components of microalgae--lipids, carbohydrates, and proteins--are amenable to conversion to fuel products through a variety of processes discussed in Section 2.4. We will develop conversion costs for three fuels that use the lipid fraction of the microalgae and two fuels that primarily use other components.

Algal carbohydrate conversion has a commercial analog in ethanol production from carbohydrates (e.g., corn). The major processing issues are the quantity and compostion of the microalgae carbohydrate and the available technology for hydrolysis of carbohydrates to sugars and subsequent fermentation to ethanol. Likewise, conversion of carbohydrates and lipids to methane gas via anaerobic digestion is a commercial technology. We therefore examine processing economics for ethanol and methane to determine the viability of using existing commercial processes for fuel production from microalgal feedstocks.

A unique aspect of the concept of fuels from microalgae is that many algae are known to accumulate cellular lipids, compounds that are similar enough to hydrocarbons to offer potential for producing highly refined fuels that could substitute directly for conventional transportation fuels. In Section 2.4 we reviewed processes that can utilize lipids to produce partial or complete substitutes for gasoline and diesel fuel. These lipid-based processes are neither highly developed nor optimized specifically for microalgal feedstocks. However, our objective to evaluate fuels from microalgae requires that we develop the best possible data on these processes to allow comparison with more conventional sources. Below, we estimate the costs of converting cellular lipids to pseudo vegetable oil (PVO) and ester fuel (both diesel substitutes) and to gasoline.

This section presents process flows and costs estimated for the five fuel options. Capital and operating costs are estimated for processing plants designed to handle the algae feedstock produced by the reference culture facility (1000 ha) discussed in Section 3.0. As with mass algal culture, a reference case for each fuel processing option is developed along with estimates of production costs. These reference fuel costs give us a perspective for measuring R\&D improvements to be developed in subsequent sections.

\subsection{Estimating Algal Processing Costs}

For each of the five fuel conversion processes in the study, the best available process and economic data have been compiled according to the steps shown in Figure 4-1. The processes selected convert the microalgae feedstock to the desired fuel products with the least amount of modification, although commercial viability depends on successful development work. Available fuel processing data are characterized by different levels of precision. These levels are listed below in order from most to least precise, and available microalgal feedstock processing data are categorized by the available level of precision. 


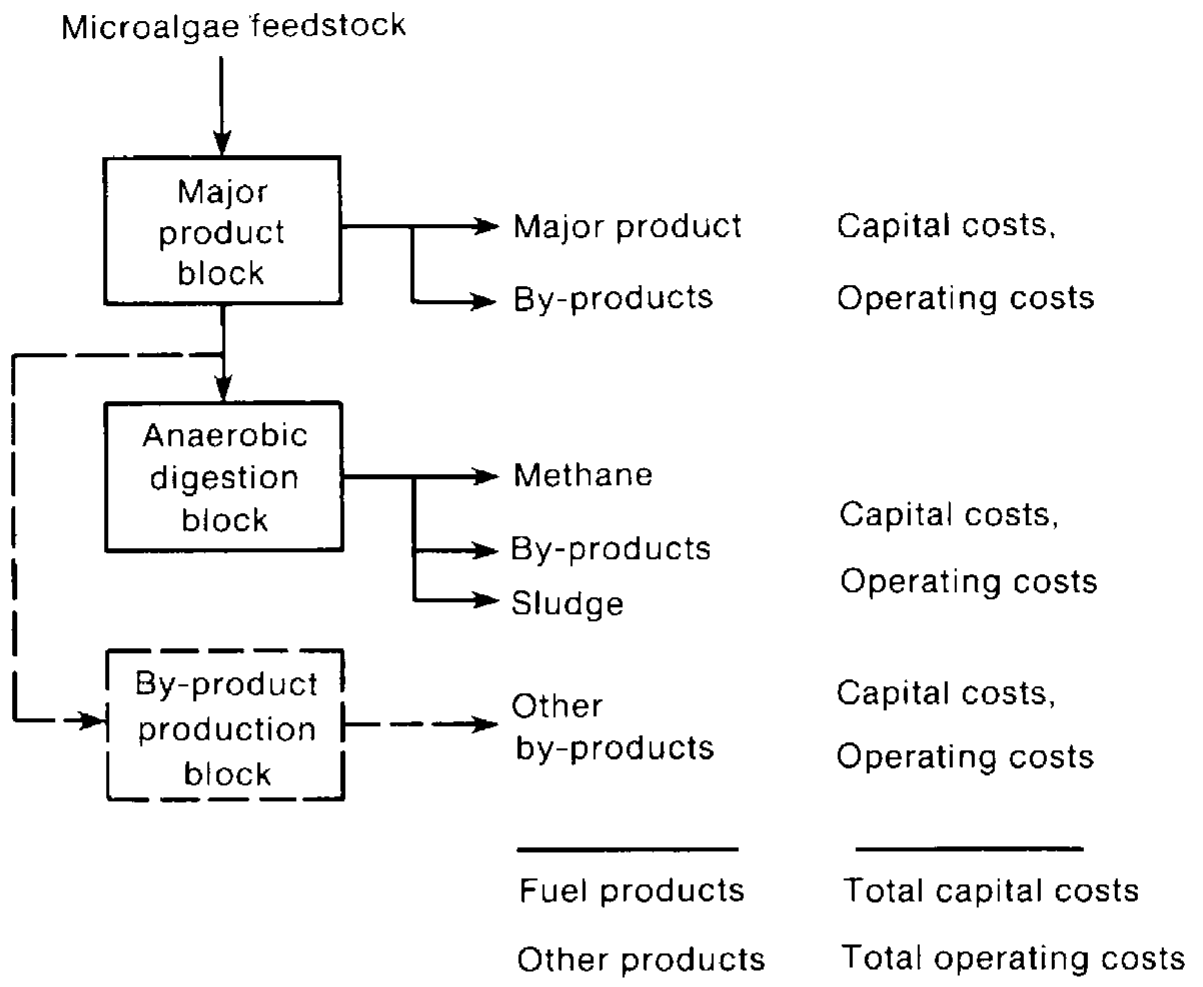

Figure 4-l. Overview of Microalgae Fuel Refining

1. Established commercial processes with precise cost and performance data for:

a. Microalgae feedstock (no processes)

b. Similar feedstock (anaerobic digestion, ethanol fermentation)

2. Detailed engineering estimates of pilot- or laboratory-scale processes with extensive data for:

a. Microalgae feedstock (PVO extraction, transesterification)

b. Similar feedstock (PVO, ester fuels)

3. Component data from analogous processes and facilities synthesized to represent microalgae applications (catalytic conversion).

Based on the available data, we constructed an estimate of capital costs (the initial fixed investment) and annual operating costs for the primary fuel process and subsequent byproduct recovery. The capital costs are expended as a lump sum at the beginning of the project; the construction period is taken as negligible compared to the facility's lifetime of 20 years. When only on-site data were available (not including support systems such as boilers and cooling water systems), the total fixed investment was adjusted by applying a contingency factor of $50 \%$ (a range of $30 \%$ to $100 \%$ is common).

Capital costs were estimated for facilities of various sizes by application of the sixtenths factor (Perry and Green 1984), which is a good estimator of economies of scale for chemical and biochemical process facilities. Scale-up by a factor of 10 would increase the capital costs by a factor of 4. All processes are designed to handle the annual algal output $(33,000 \mathrm{t})$ of the reference 1000 -ha production facility. 
Operating costs consist of the following major components: raw materials, utilities (electric power, steam, and cooling water), maintenance, labor and supervision, taxes, depreciation (10 year, straight line), and return on investment (15\%, based on a debt/equity ratio of 3). Below we summarize capital and operating costs for the five fuel production options. The lipid-based processes are discussed first followed by processes that do not rely solely on the lipid content of the algal feedstock.

\subsection{Lipid-Based Conversion Routes}

\subsection{Pseudo Vegetable Oil}

Figure 4-2 is a simplified flow diagram of a process to produce PVO from microalgae. No chemical reactions are involved; the components recovered are already present in the algae in a state suitable for diesel fuel.

This process has been adapted from one used comercially on soybeans (Adams et al. 1981). Its success depends on identification of a suitable solvent; the hexane used in the soybean process might extract other algal lipids (e.g., glycolipids) that are detrimental to the process, resulting in lower conversion rates. Process optimization requires the development of a selective solvent to extract from microalgae cellular lipids a fuel that has the desired properties.

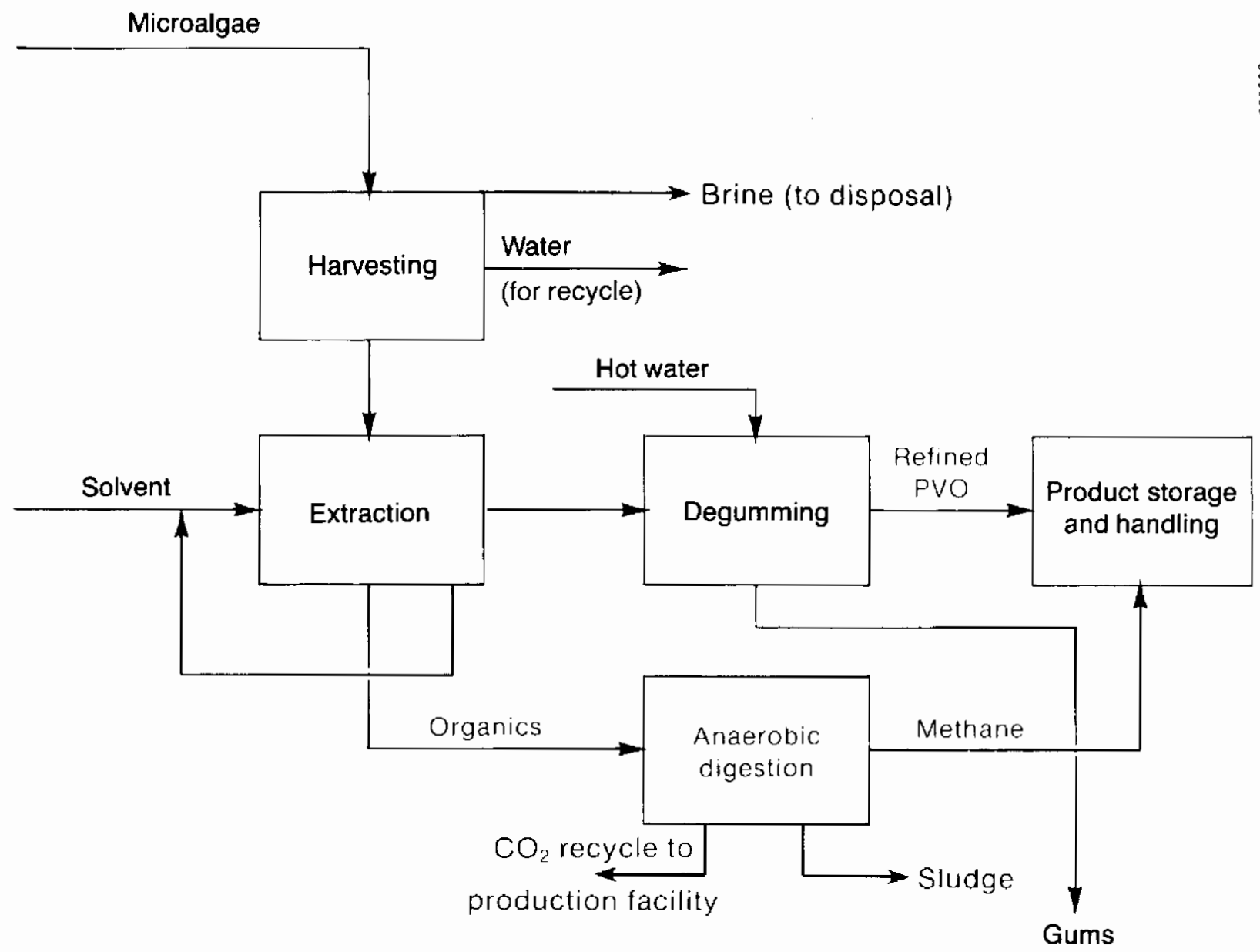

Figure 4-2. Pseudo-Vegetable Oil (PVO) Production Process Generalized Flow Diagram 
The lipids not extracted and the remaining portions of the algae (carbohydrates and protein) are anaerobically digested to produce methane and carbon dioxide. Alternatively, the protein is recovered and used as animal feed, and only the carbohydrates are digested. Table 4-1 summarizes mass and energy data estimated for the PVO process based on processing the feedstock of the reference algal culture facility $(31,000 \mathrm{t}$ annually of microalgae biomass with $30 \%$ lipid content).

\subsubsection{Transesterification}

Figure 4-3 shows how the transesterification process might be adapted to producing fuels from microalgae (Kusy 1982; Freedman and Pryde 1982; Technical Insights Inc. 1980). This part of the process is noticeably similar to the PVO extraction process because of the front-end solvent lipid extraction. However, the rest of the process, including the reactor itself, is more complex because lipid extracts, used directly in PVO conversion, are chemically modified by the addition of methanol or ethanol. Substitution of 3 moles of methanol (or ethanol) for 1 mole of glycerol yields glycerol that is recovered as a byproduct.

\subsubsection{Catalytic Conversion}

The Mobil methanol-to-gasoline process, as adapted to crude algal lipids, is shown in Figure 4-4 (Weisz et al. 1979; Voltz et al. 1976). A pre-extraction step, similar to those mentioned for the PVO and ester processes, would probably make higher conversion efficiencies possible. Again, details of the solvent system and extraction process are preliminary and depend on process optimization reactor studies. Calculated production data for the reference 1000-ha refinery are presented in Table 4-1; the methane quantity represents the total from catalytic conversion (5\% of the total) plus anaerobic digestion ( $95 \%$ from unreacted organics).

\subsection{Nonlipid-Based Processes}

\subsubsection{Anaerobic Digestion}

Figure 4-5 shows schematically the anaerobic digestion process as adapted for microalgae (Ashare and Wilson 1979). As discussed earlier, the organic fractions of the algae (all components except ash) are anaerobically digestible, so once the algae has been harvested, little if any pretreatment is required. Note that the other fuel processing options also utilize anaerobic digestion and produce methane as a secondary product. The biogas product typically contains $60 \%$ methane and $40 \%$ carbon dioxide by volume. The liquid effluent contains soluble nitrogen from the original algal proteins; the nitrogen can be recovered in the form of ammonia for recycle to the culture. Table 4-1 summarizes the various product yields from anaerobic digestion.

\subsubsection{Ethanol}

Figure 4-6 is a flow diagram of an ethanol production process adapted from a corn-based process (Ralph Katzen Associates 1980; Technical Insights Inc. 1980). Some details of the process would change for a $10 \%$ algal slurry; for example, the mash cooking step has somewhat higher energy requirement because of the amount of water present. The fungal amylase enzyme is added to the cooked mash to convert the starch polymer to monomeric sugar units. The fermentation products are separated by distillation, and all nonfermentables are discharged out the bottom of the still. From a corn substrate, the 
Table 4-1. Reference Production Summary for Microalgae Processing Options

Basis: 1000-ha algal culture facility

$33,000 \mathrm{t}$ of algal biomass processed annually

$7 \%$ photosynthetic efficiency

$30 \%$ algal lipids

\begin{tabular}{|c|c|c|c|c|c|}
\hline \multirow{2}{*}{ Process Products (units) } & \multicolumn{5}{|c|}{ Major Fuel Products } \\
\hline & PVO & Ester Fuel & Gasoline & Methane & Ethanol \\
\hline $\begin{array}{l}\text { Major fuel production } \\
\qquad\left(10^{6} \mathrm{gal} / \mathrm{yr}\right)\end{array}$ & 2.1 & 2.1 & 1.9 & (see below) & 1.2 \\
\hline $\begin{array}{l}\text { Other products } \\
\text { Methane }\left(10^{12} \mathrm{~J} / \mathrm{yr}\right)\end{array}$ & 600 & 600 & 510 & 870 & 690 \\
\hline $\mathrm{CO}_{2}\left(10^{6} \mathrm{~m}^{3} / \mathrm{yr}\right)$ & 11 & 11 & 9 & 16 & 14 \\
\hline $\begin{array}{l}\text { Nitrogen }\left(10^{3} \mathrm{t} / \mathrm{yr}\right. \\
\left.\text { as } \mathrm{NH}_{3}\right)\end{array}$ & 2.4 & 2.4 & 2.2 & 2.7 & 2.3 \\
\hline Glycerol (t/yr) & -- & 760 & -- & - & -- \\
\hline $\begin{array}{l}\mathrm{LPG}\left(10^{12} \mathrm{~J} / \mathrm{yr}\right) \\
\quad\left(10^{3} \mathrm{gal} / \mathrm{yr}\right)^{\mathrm{a}}\end{array}$ & - & $\begin{array}{l}-- \\
-\end{array}$ & $\begin{array}{r}90 \\
840\end{array}$ & - & - \\
\hline Diesel $\begin{aligned}\left(10^{12} \mathrm{~J} / \mathrm{yr}\right) \\
\left(10^{3} \mathrm{gal} / \mathrm{yr}\right)\end{aligned}$ & -- & -- & $\begin{array}{r}43 \\
320\end{array}$ & $\begin{array}{l}-- \\
--\end{array}$ & -- \\
\hline
\end{tabular}

as butane, 103,000 Btu/gal

components would be dried and sold as distiller's dried grains (DDG), slop, or stillage. Fermentables represent a much smaller fraction (13\%) of the total algae biomass than they do in corn (65\%), so separation of the nonfermentables before or during the mash cooking step might be economically desirable. Table 4-1 shows the various products from the ethanol-from-microalgae process. About $85 \%$ of the carbon dioxide produced is derived from anaerobic digestion; the remainder is derived from the fermentation unit.

\subsection{Summary of Fuel Processing Costs}

Table 4-2 and Figures 4-7 and 4-8 summarize the components that contribute to gross operating costs of each of the five reference fuel production processes based nn processing the output of the 1000-ha reference facility. The cost components shown are raw materials (not including the microalgae feedstock itself), utilities, labor, maintenance, taxes, and depreciation. Note that labor and depreciation are the primary components in every case. These refining operations would be considered highly capital-intensive; only in the PVO process does depreciable capital contribute less than $30 \%$ to the gross operating cost. The efficiency of capital in these processes could be improved by (1) further process development, including equipment design, to improve the operating efficiency, or (2) a centralized refinery that serves a number of algal ponds; the larger refinery would realize significant economies of scale, as described by the six-tenths factor. The fuel processing plant sizes associated with the 1000-ha algal facility are well below their optima for efficient, intensive use of capital. The large labor and maintenance components might also be reduced in a full-scale process. 


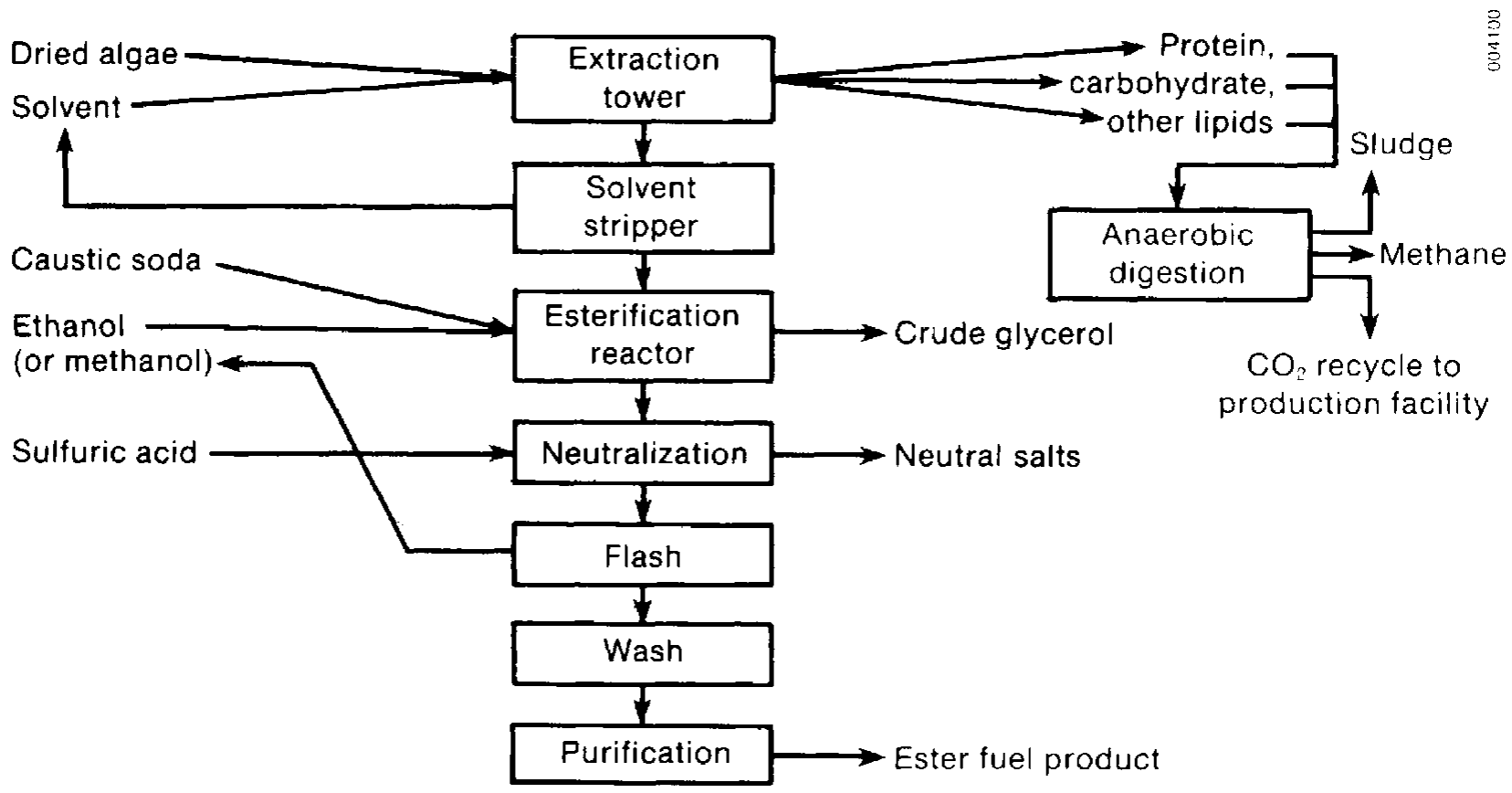

Figure 4-3. Schematic of Transesterification Process

\subsection{Fuel By-product Values}

Final product and by-product values for all fuel processing options are fixed according to estimates of year 2010 prices. Price forecasts are taken from DOE/BETD value-based cost goals that represent estimates of prices that alternative technologies must achieve to be competitive in 2010 .

Fuel cost goals are presented in Table 4-3 for all the primary fuels under consideration. Prices of gasoline, ethanol, methane, and diesel fuel are taken directly from the cost goals. Other primary fuel prices are derived by adjusting the fuel price according to its Btu content relative to a similar fuel. Prices of pseudo vegetable oil and ester fuels are derived from the projected price of diesel fuel, and the price of fuel gas from the projected price of methane.

By-products from the various primary fuel processing options and their assumed value are listed in Table 4-4. In this table all fuel by-products are valued at their cost goal level. Values for other by-products are set at their current market price since year 2000 projections for these products are not available. Thus, total revenues from processing are underestimated by the real price increase expected for these by-products. In the absence of consistent projections for nonfuel by-products, we chose to err on the side of conservatism in evaluating fuel option feasibility.

\subsection{Reference Fuel Production Costs}

So far we have described the basic elements of fuel processing facilities, described the range of parameters for each element, and established a reference system for the five processes. We now can combine reference microalgae production and fuel with 


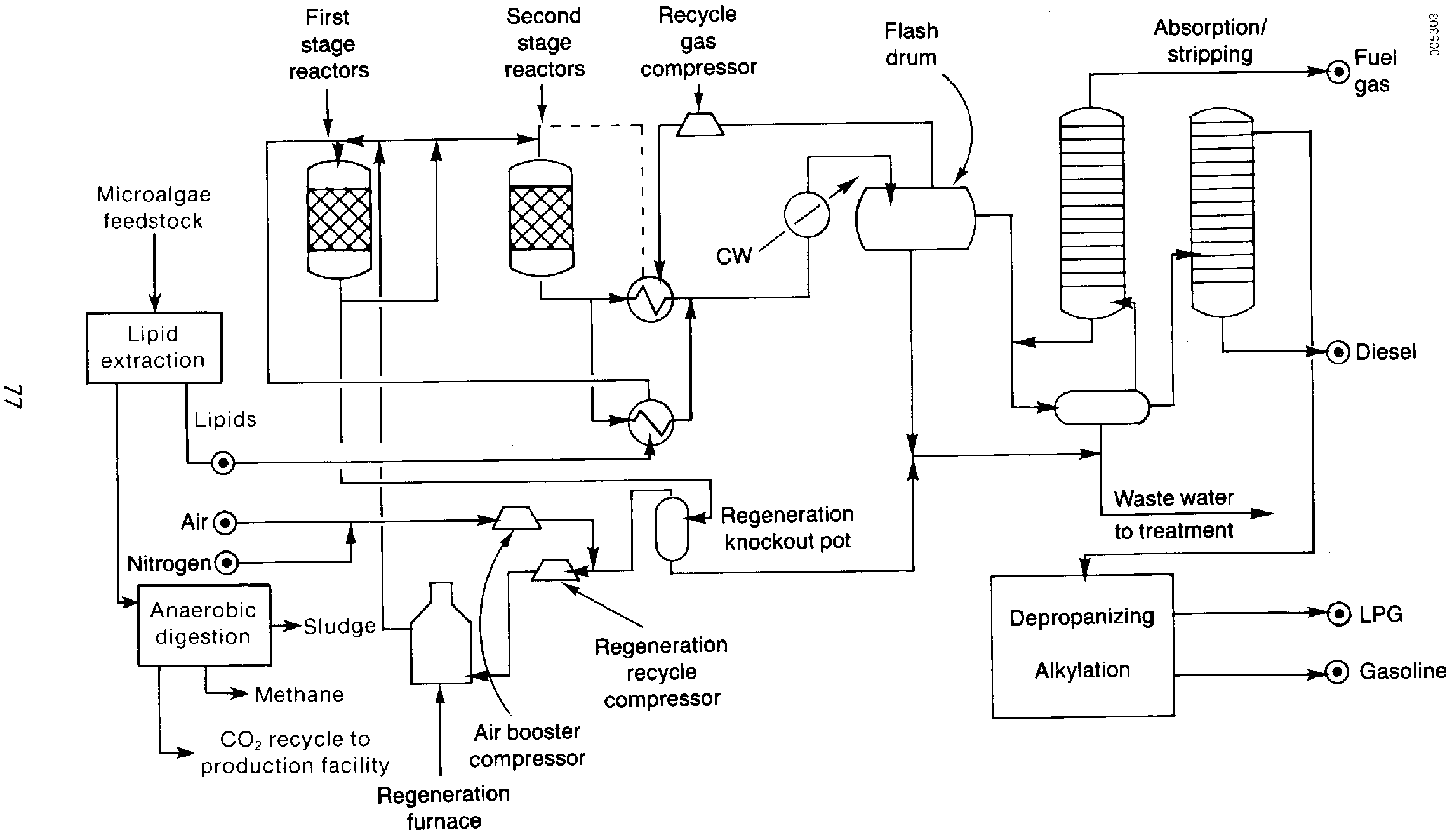

Figure 4-4. Mobil M-gas Process Adapted for Lipid Oils 


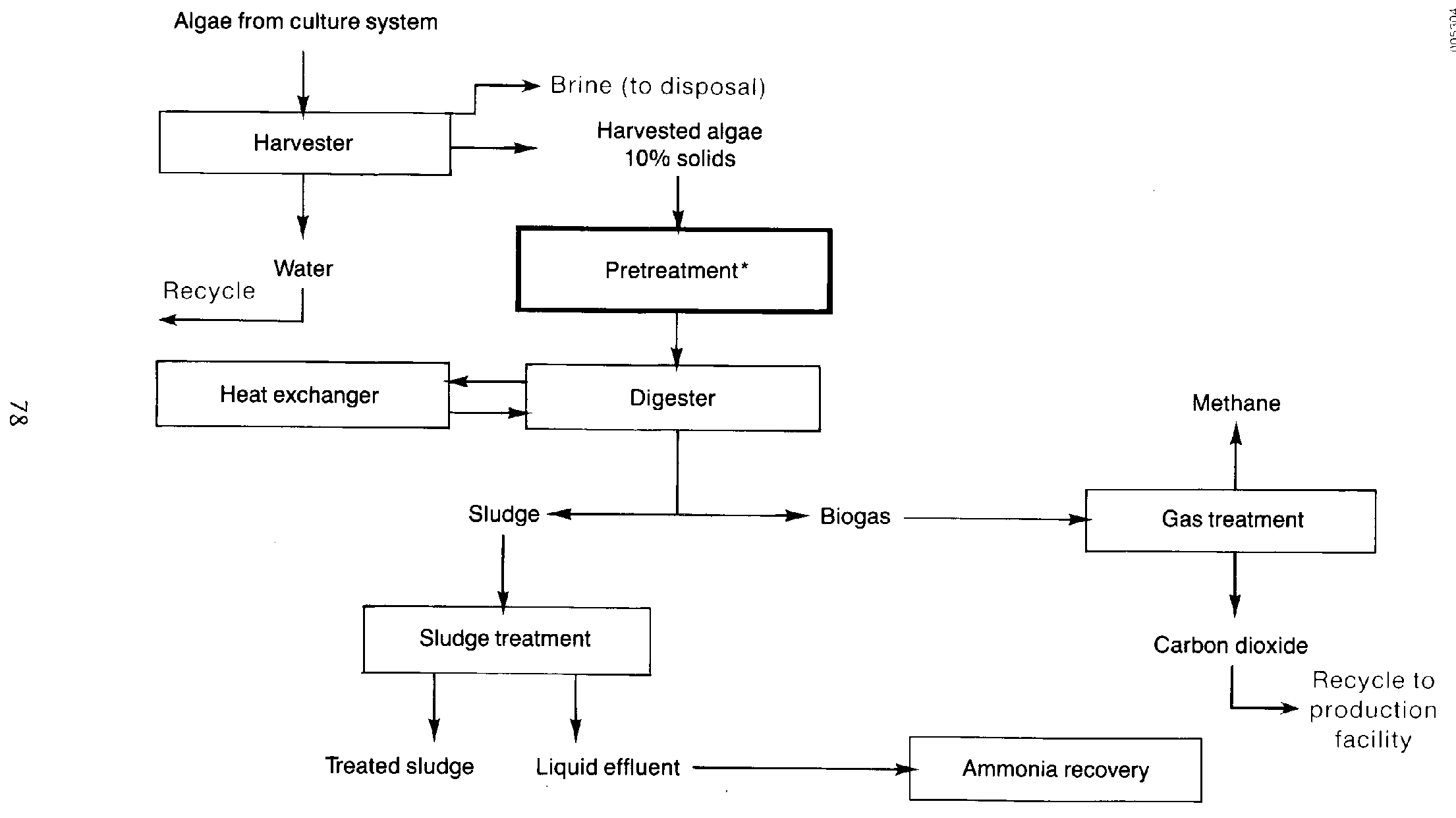

*Optional operation

Figure 4-5. Anaerobic Digestion of Microalgae 


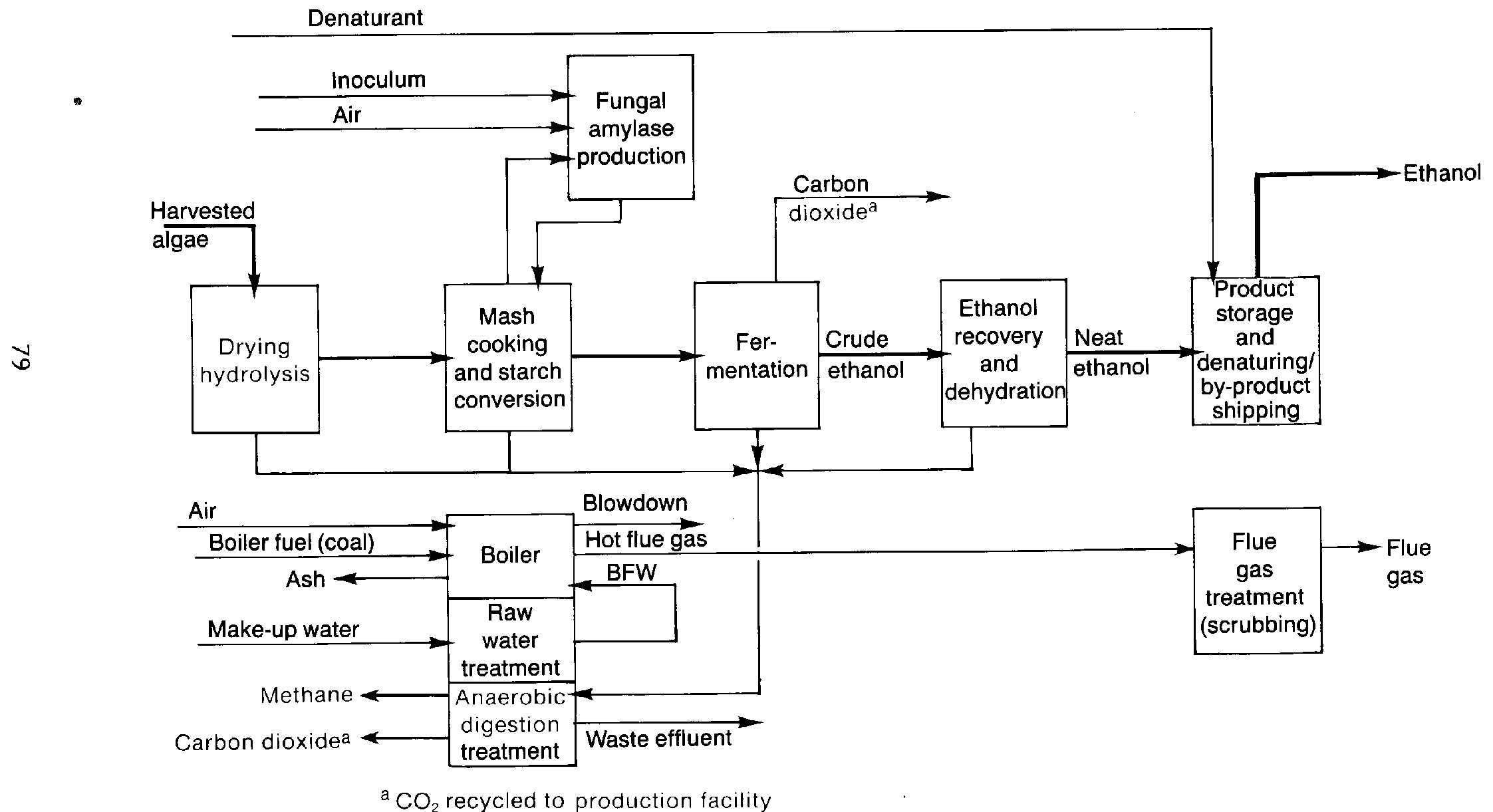

Figure 4-6. Ethanol Production Process Generalized Flow Diagram 
Table 4-2. Summary of Reference Capital, Operating, and Allowable Feedstock Costs for Fuel Processing Options

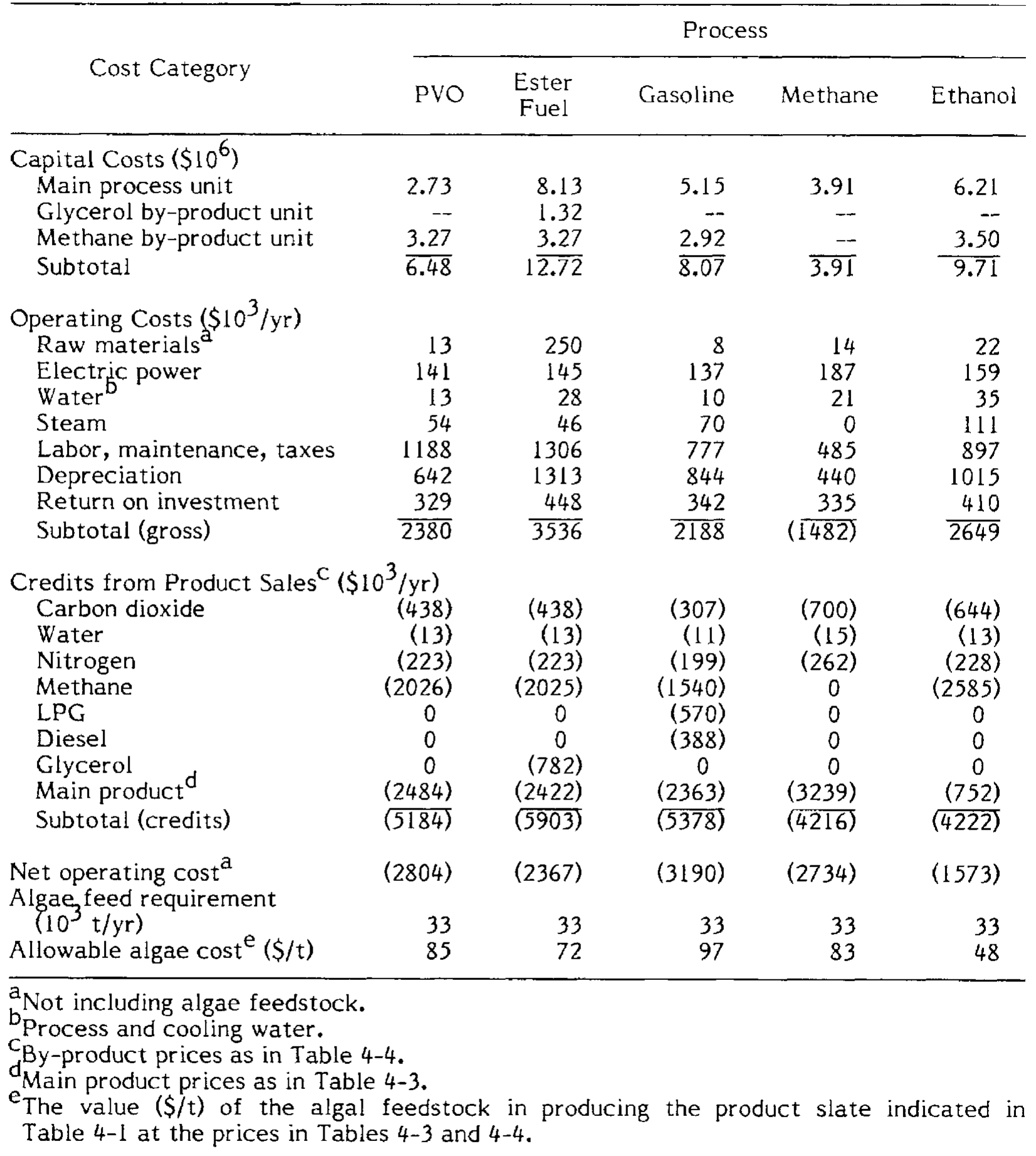



Anaerobic digestion
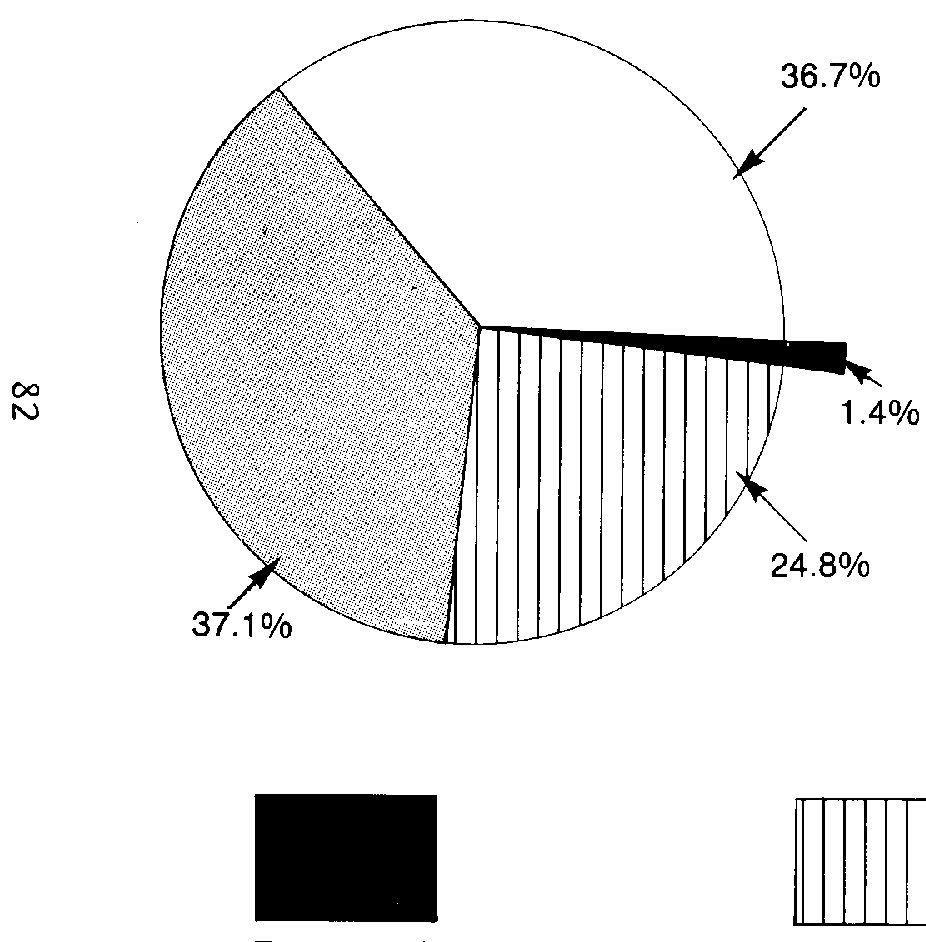

Raw materials

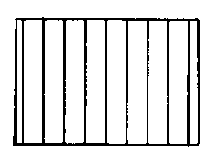

Utilities
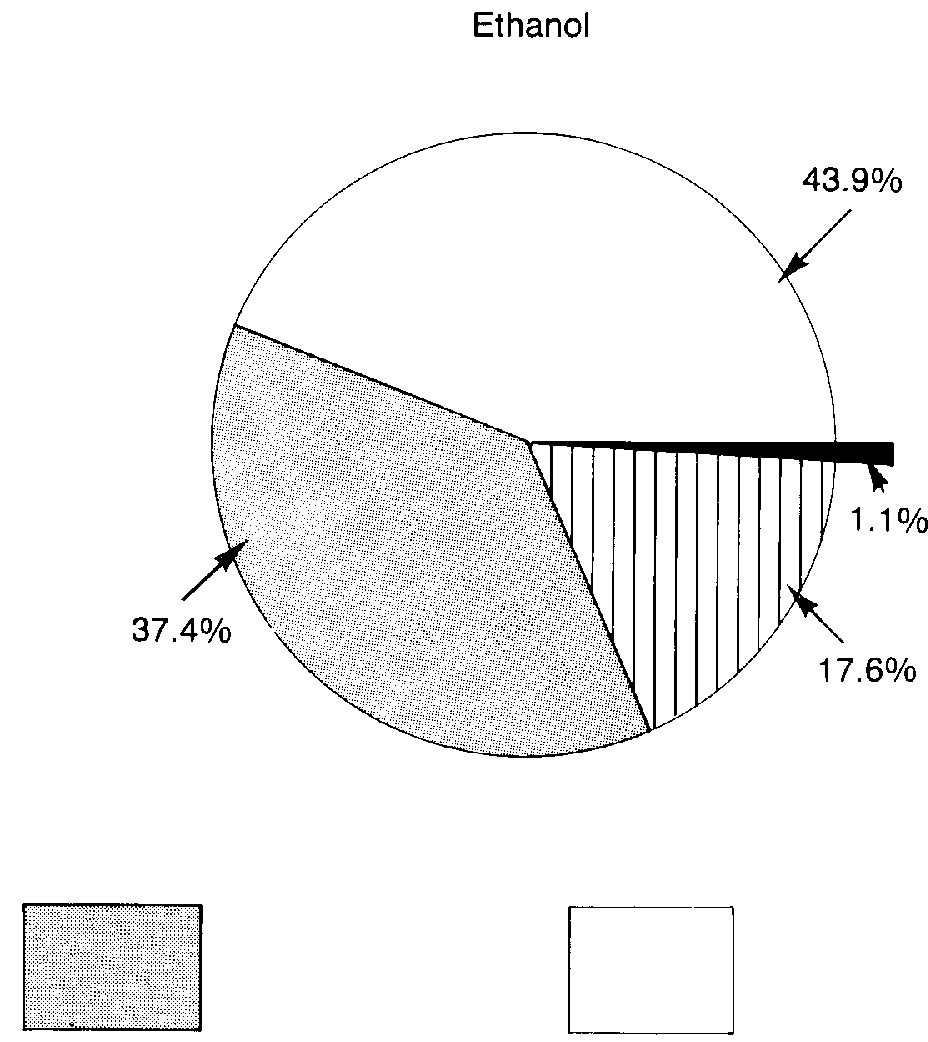

Labor,

maintenance,

taxes 
Table 4-3. Estimated Year 2010 Fuel Cost Goals for Microalgae Products

\begin{tabular}{lcc}
\hline & \multicolumn{2}{c}{ Project Price } \\
\cline { 2 - 3 } Fuel Product & $\$ / 10^{6}$ Btu & $\$ /$ gal $^{\mathrm{a}}$ \\
\hline Gasoline & 16.26 & 1.75 \\
Diesel fuel & 16.26 & 1.75 \\
Methane & 7.40 & -- \\
Fuel gas & 7.40 & -- \\
LPG & 7.40 & -- \\
Ethanol & -- & 1.20 \\
PVO fuel & -- & 1.75 \\
Ester fuel & -- & 1.75 \\
\hline a Estimated from Btu value using & lower heating \\
\multicolumn{2}{c}{ value of the liquid fuel. }
\end{tabular}

Table 4-4. Algal Fuel Processing By-products and Values

\begin{tabular}{|c|c|c|c|}
\hline Primary Fuel & ByProducts & Value (units) & Utilization $^{a}$ \\
\hline Ethanol & $\begin{array}{l}\mathrm{CO}_{2} \\
\text { Methane } \\
\text { Methane by-products }\end{array}$ & $\begin{array}{l}\$ 0.07\left(10^{3} \mathrm{~m}^{3}\right) \\
\$ 7.40\left(10^{6} \mathrm{Btu}\right) \\
\text { (see below) }\end{array}$ & $\begin{array}{l}\text { Captive } \\
\text { Export }\end{array}$ \\
\hline PVO & $\begin{array}{l}\text { Methane } \\
\text { Methane by-products }\end{array}$ & $\begin{array}{l}\$ 7.40\left(10^{6} \mathrm{Btu}\right) \\
\text { (see below) }\end{array}$ & Export \\
\hline Ester Fuel & $\begin{array}{l}\text { Glycerol } \\
\text { Methane } \\
\text { Methane by-products }\end{array}$ & $\begin{array}{l}\$ 1.54(\mathrm{~kg}) \\
\$ 7.40\left(10^{\circ} \mathrm{Btu}\right) \\
\text { (see below) }\end{array}$ & $\begin{array}{l}\text { Export } \\
\text { Export }\end{array}$ \\
\hline Methane & $\begin{array}{l}\mathrm{CO}_{2} \\
\text { Nitrogen }\left(\mathrm{NH}_{3}\right) \\
\text { Supernatant }\left(\mathrm{H}_{2} \mathrm{O}\right) \\
\text { Sludge }\end{array}$ & $\begin{array}{l}\$ 0.07\left(10^{3} \mathrm{~m}^{3}\right) \\
\$ 183(\mathrm{t}) \\
\$ 0.06\left(\mathrm{~m}^{3}\right)\end{array}$ & $\begin{array}{l}\text { Captive } \\
\text { Captive } \\
\text { Captive } \\
\text { Disposal }\end{array}$ \\
\hline Gasoline & $\begin{array}{l}\text { Fuel gas } \\
\text { LPG } \\
\text { Diesel fuel } \\
\text { Methane } \\
\text { Methane by-products }\end{array}$ & $\begin{array}{l}\$ 7.40\left(10^{6} \mathrm{Btu}\right) \\
\$ 7.40\left(10^{6} \mathrm{Btu}\right) \\
\$ 13.30\left(10^{6} \mathrm{Btu}\right) \\
\$ 7.40\left(10^{6} \mathrm{Btu}\right) \\
(\text { see above })\end{array}$ & $\begin{array}{l}\text { Export } \\
\text { Export } \\
\text { Export } \\
\text { Export }\end{array}$ \\
\hline
\end{tabular}


processing costs to calculate the reference case fuel production cost. We also have noted that cost goals for fuels have been developed for biomass technologies. The purpose of these cost goals is to fix year 2010 prices at levels consistent with expected supply and demand for energy. Technology evaluations use these goals as fixed targets for directing research and evaluating program progress.

We use these cost goals to aid our evaluation of microalgae technology by assuming that all fuel products are sold at their cost goal level (Table 4-3). In addition, we assume that by-products are sold at the values indicated in Table 4-4. Since revenues are fixed when product and processing streams are identified, to determine technology status we must compare the cost of producing a microalgae feedstock with its value in the production of fuels and by-products of fixed value.

The measure of cost-effectiveness we utilize for the reference case, and in later sections to evaluate the benefits of $\mathrm{R} \& \mathrm{D}$ improvements, is a comparison of production cost with the allowable processing cost. The allowable processing cost is the maximum value of (or amount a facility operator would pay for) microalgal feedstock used to produce a slate of products and by-products valued at the cost goal level. To calculate reference allowable processing costs, we use the feedstock composition and quantity of the reference algal biomass as inputs to each fuel process. We then calculate product yields according to data for the reference process described above. Capital costs are fixed by the assumed plant size, and operating costs are calculated according to the amount of material processed.

Annualized processing costs must be offset by revenues from the sale of products, so we estimate total revenues from the sale of all products, subtract the annualized capital and operating costs, and assume that the algal feedstock is free. The difference between revenues and costs is the maximum value of the feedstock. Dividing this value by the total amount of feedstock processed yields the allowable processing cost for the feedstock-the maximum amount a facility operator would pay for it. This allowable cost must be equal to or less than the production cost for competitive fuel production from microalgae.

Allowable processing feedstock costs for the reference production and fuel systems are listed in Table 4-5. The large difference between estimated algal feedstock production cost and its value in fuel production illustrates the nascent stage of technology development. In the following sections we identify specific areas where technology can be improved and calculate the extent to which these improvements narrow the gap between production cost and processing value. Just as we chose specific parameter values to derive reference production and processing cost, the comparison of reference cost and value serves to place $R \& D$ improvement in perspective. The reference comparison shows that fuel production from microalgae currently is not commercially viable.

In the next sections we evaluate how the gap between production and allowable processing cost might be closed and how a cost-effective technology can be developed by evaluating production cost improvements. We then evaluate processing improvements and combine them into an attainability concept that embodies achievement of the cost goals through identifiable, attainable R\&D improvements. 
Table 4-5. Comparison of Feedstock Production Cost and Allowable Processing Cost for the Reference Case

\begin{tabular}{lcc}
\hline & \multicolumn{2}{c}{ Reference Case } \\
\cline { 2 - 3 } Fuel Processing Option & $\begin{array}{c}\text { Feedstock } \\
\text { Production } \\
\text { Cost } \\
(\$ / \mathrm{t})\end{array}$ & $\begin{array}{c}\text { Allowable } \\
\text { Processing } \\
\text { Feedstock Cost } \\
(\$ / \mathrm{t})\end{array}$ \\
\hline Ethanol & 393 & 64 \\
Methane & 393 & 90 \\
Pseudo vegetable oil (PVO) & 393 & 85 \\
Ester fuel & 393 & 95 \\
Gasoline & 393 & 102 \\
\hline
\end{tabular}

Note: All parameters for feedstock production and processing are set at the reference value, and all products and coproducts are valued at their cost goal value. All costs are in 1984 \$. The feedstock production cost is the reference case cost of producing microalgae of $30 \%$ lipid content. The allowable processing cost is the value of the algal feedstock to a processing facility faced with fuel and bymproduct prices listed in Table 4-4. 


\subsection{INITIAL SCREENING OF COST CONTRIBUTIONS}

To evaluate the effect of changes in microalgal feedstock cost from the reference value due to changes in individual parameters, a sensitivity analysis was conducted for each parameter discussed in the previous section. The parameters were grouped into four general areas--resources, biology, facility design, and financial--to permit relative comparisons among similar parameters. In this way, those parameters that significantly affect product cost can be identified. These "high sensitivity" parameters within each general area become a subset of parameters for subsequent examination. Our objective is to first screen the numerous variables to identify those with the greatest impact on production cost. Then we examine the effect of parameter changes that can be associated with specific improvements where several parameter values change, not just one.

Sensitivities were derived in a static-comparative manner--parameter values for each variable were varied over the ranges developed in Section 3.0 holding all other variables at their reference value. Thus, they measure the relative sensitivity of each variable, not groupings of related variable changes. In the next section we will examine optimizations of the system by adjusting or improving groups of functionally interrelated effects.

Two other methodological points should be kept in mind in evaluating the sensitivities. First, the range of sensitivity for each variable corresponds to those established in Section 3.0 as representative of current technology status and likely (achievable) and worst case values. Thus, in comparing sensitivities among variables, the differences do not represent numerically symmetric variations (e.g., $10 \%$ or $20 \%$ from the reference value) from the base case. Instead, relative sensitivity values compare likely outcomes derived from the set of technical or economic possibilities.

Second, some variables cannot be independently varied due to technical interrelationships. Carbon dioxide supply cost is calculated as a fixed and variable cost with scale economics. Thus, parameter changes that induce production variability increase or decrease the amount of carbon dioxide required and therefore costs change. We have not adjusted sensitivity values of algal production cost for these variations as they are inexorable and representative of the technology. Other variables are subject to summation constraints; e.g., the sum of the proportions of lipid, carbohydrate, nitrogen, ash, and intermediate content of cells must equal one. Thus, changing one requires adjustments in all others. These variables are varied as a complete set maintaining a viable biochemical cell composition.

In summary, sensitivities presented in this section serve to evaluate the effect on algal production cost of prescribed variations in the levels of key variables. These sensitivities are useful to identify factors whose level have a large impact on algal production cost and to establish priorities for reducing cost.

\subsection{Resource Parameter Sensitivity}

Thirteen of the 14 resource input assumptions were varied under the general category of resource parameters; 2 only the assumed daily solar constant, established at a reference value of $5000 \mathrm{kcal} / \mathrm{m}^{2} / \mathrm{d}$, was not varied. This reference value for the daily solar constant represents average sunlight available in the Southwest and is used to calculate the photosynthetic efficiency of the algal based on gross yield within the culture. Since we evaluate the region in general rather than specific sites, we hold this variable constant at a level representative of the region, thus taking the solar resource as given. 
The resource parameter sensitivities are presented in Figure 5-1. We have identified three major areas in which resource issues significantly affect feedstock cost that can be categorized as water, nutrients, and energy.

The water parameters are interrelated. Based on the reference system values and sensitivity ranges for these parameters, evaporation, salinity of the source water, and water supply costs are the major variables affecting product cost. Maximum evaporative losses in the microalgae facility can increase product costs $12 \%$ from the reference value of $\$ 393 / \mathrm{t}$. However, even if evaporative losses were significantly reduced to $0.002 \mathrm{~m} / \mathrm{d}$, the effect on product cost is smaller $(-3 \%)$ because acquisition costs for makeup water remain high and there are other nonevaporative water losses. The cost of water can have a large impact on algal costs and will be largely determined by local resource conditions. Although some individual water parameters do not have a large impact on reference production cost, their combined impact can be greater. In Section 6.0 we examine water linkages in detail and derive an integrated perspective on water issues; that is, we relate all sets of water variables to conditions likely to be encountered in practice.

The major nutrient expenses for the reference system are carbon dioxide and nitrogen (ammonia). Carbon and nitrogen requirements and costs are determined by gross algal productivity minus the available supply in the source water and the unit costs for each nutrient. A more nutrient-rich source water can reduce nutrient cost (3\% for nitrogen and $7 \%$ for carbon) but these benefits are tied to particular water resource characteristics and may be limited in availability.

Energy costs also affect product costs for the reference system. While this parameter is less significant than water and nutrients, the large energy requirement for the facility indicates that a low-cost electrical energy supply is beneficial and should be considered during facility siting and design.

Of all the parameters shown in Figure 5-1, six warrant additional analysis because their influence on product cost is profound. These parameters are evaporation, salinity of the source water, carbon content of the source water, energy costs, water supply costs, and the distance carbon dioxide must be transported to the microalgae production facility.

\subsection{Facility Design Parameter Sensitivity}

Of the 21 design-related parameters used in the model, 15 parameters are varied. Figure 5-2 presents the sensitivity of product cost to changes in these 15 parameters. Parameters that were not varied include basic pond geometry (length-to-width ratio set at 10 in the reference system), facility size (fixed at a nominal value of $1000 \mathrm{ha}$ ), efficiency of the drive system for mixing the culture system (90\%), and the minimum solids concentration of the product stream (10\%).

Effective culture area and effective operational downtime are design features that directly affect the productivity of the system; improvements in either parameter increase the overall productive area for the facility, thereby increasing the total net yield. Changes in mixing velocity, mixing drive efficiency, and depth change the energy required to move or pump the culture water and hence energy costs for the system. 


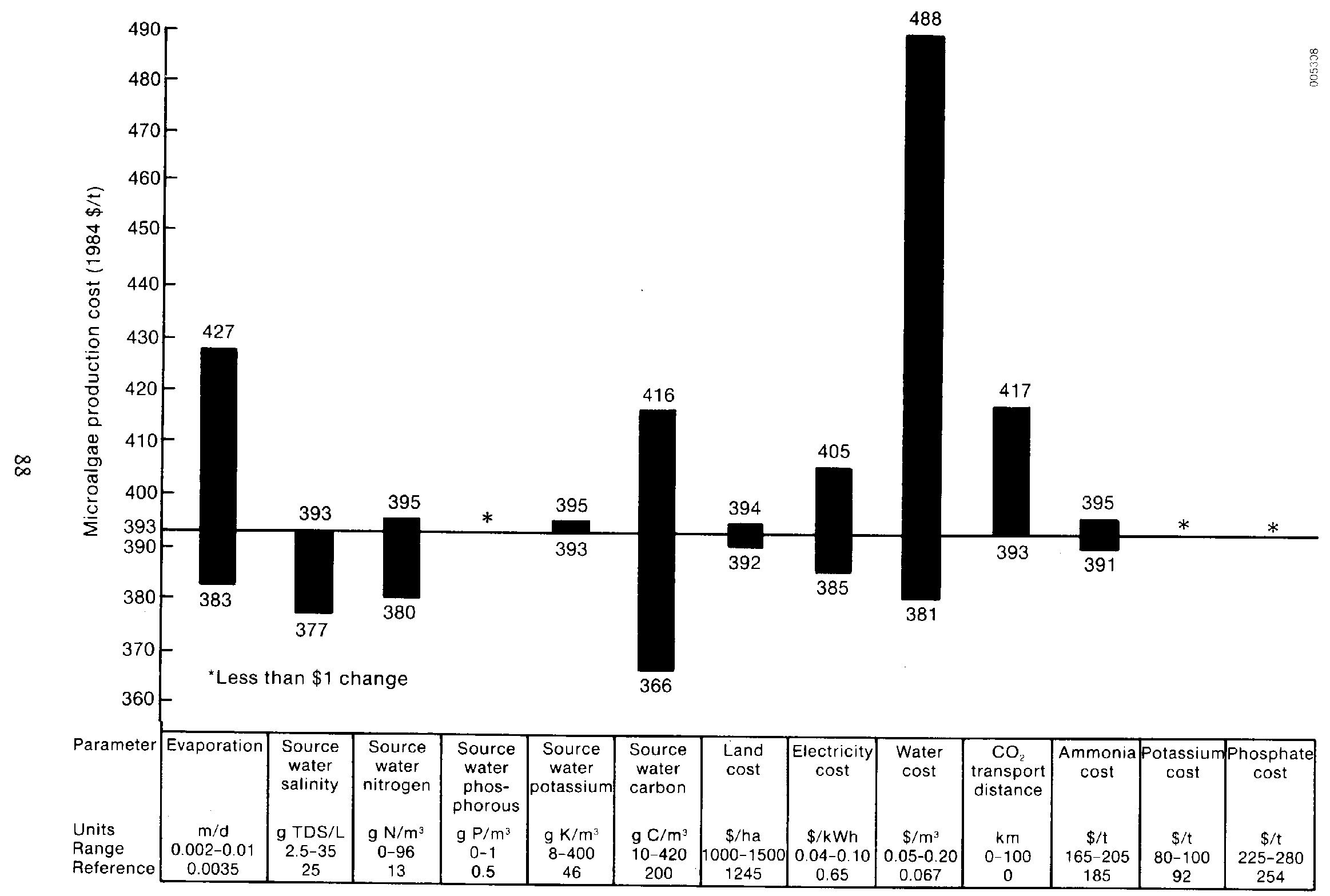

Figure 5-1. Resource Parameter Sensitivity 


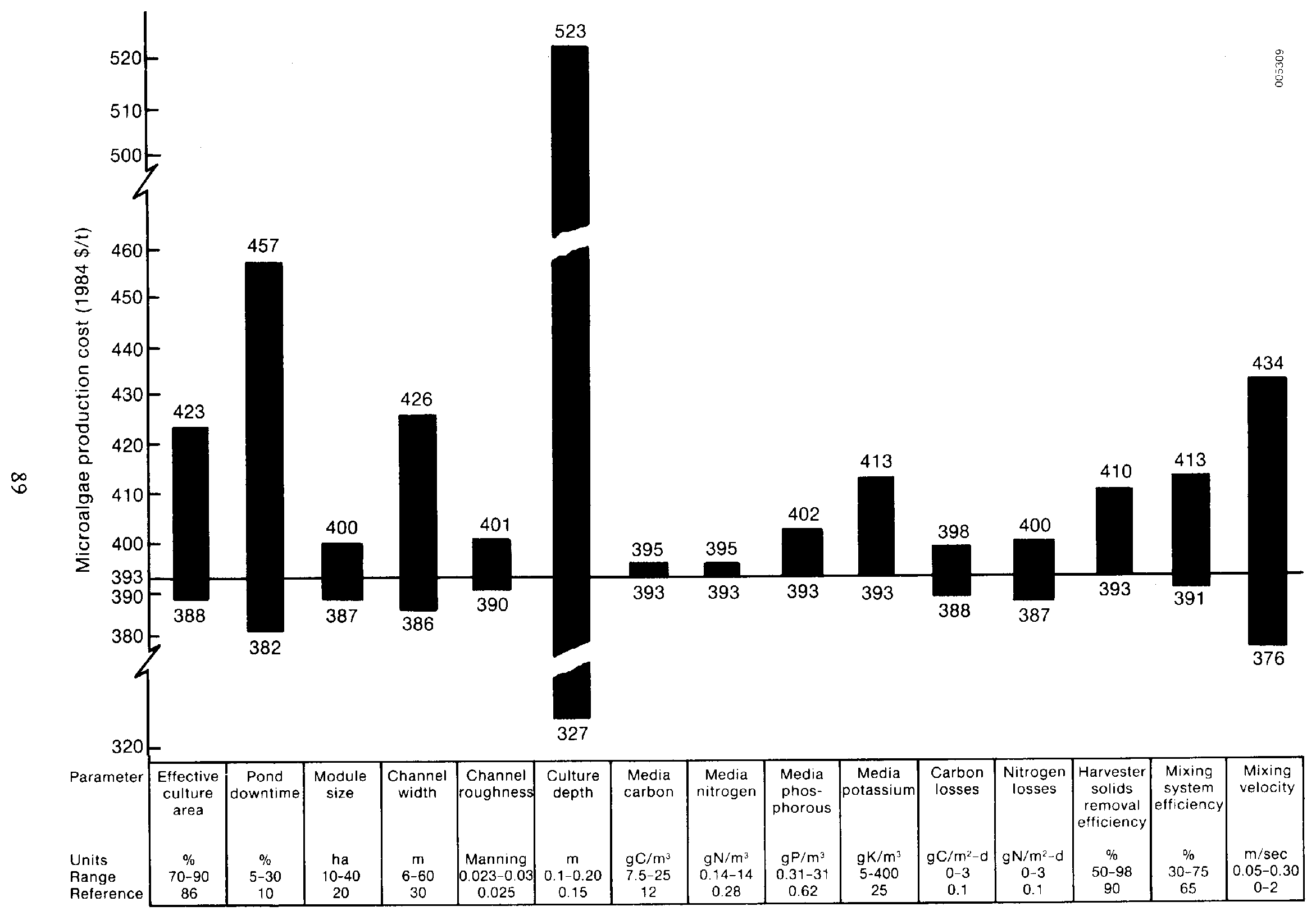

Figure 5-2. Facility Design Parameter Sensitivities 
Similarly, carbon and nitrogen losses due to outgassing from the culture surface are significant design parameters also affected by resource cost issues. Finally, the type of harvester system or the efficiency of solid removal by any harvester system affects final product cost.

The important facility design parameters influencing product costs are effective culture area, downtime, channel width, mixing velocity, culture depth, carbon and nitrogen outgassing losses, mixing system pump efficiency, and harvester solids removal efficiency.

\subsection{Biology Parameter Sensitivity}

The model utilizes 18 parameters to define the biological relationships of the mass culturing microalgae. Of this total, many parameters are fixed because they are typically well understood and are used by the model to calibrate other system effects. Examples of such values are minimum nitrogen cell content $(0.03 \mathrm{~g} / \mathrm{g}$ dry weight), nutrient content of the cell, and energy content of the various cell contents. Parameters such as lipid, carbohydrate, and protein content and parameter values used to calculate algal productivity must be varied as a group to maintain consistency among related variables. Independent variation of any one parameter was avoided to prevent unrealistic single parameter values. The biological parameters affecting feedstock cost are presented in Figures 5-3 and 5-4. The sensitivity of nonproductivity related parameters in Figure 5-3 indicates that salinity tolerance and growing season have a large effect on feedstock costs.

Sensitivities for detention time, depth, and density are illustrated in Figure 5-4. Functionally interrelated in nature and in the model, these factors are each associated with common aspects of the technology. Increased depth at constant density has a corollary in increased density and a fixed depth. However, achievement of improvements in either are tied to the characteristics of algal species and to the design of the facility. Thus, not all combinations represented in the sensitivities are feasible when viewed in light of species characteristics. However, the sensitivities do proxy for the dramatic cost reductions possible if species can be developed to live in denser cultures at greater depths. Likewise, decreased detention time illustrates enhanced productivity, a derivative of improved species efficiency. In the next section we will summarize these effects through low mass productivity to illustrate more clearly possible improvements in the technology.

\subsection{Financial Parameter Sensitivity}

Financial parameter sensitivities illustrated in Figure 5-5 capture the effect of a variety of economic uncertainties associated with any large construction project. As is customary in technology evaluations, real cost increases for basic inputs are assumed zero. All costs are measured in real (constant 1984) dollars and assuming constant real costs is consistent with assuming that a microalgae project, by itself, would not tax resources sufficiently to induce real price increases and that the facility uses materials not expected to incur price inflation above the general rate of inflation. To illustrate the impact of relaxing these assumptions, we calculated algal production costs at $2 \%$ real price increases, throughout the period of construction and operating period for capital, operating, and maintenance costs. The effect is most severe for operating costs, 


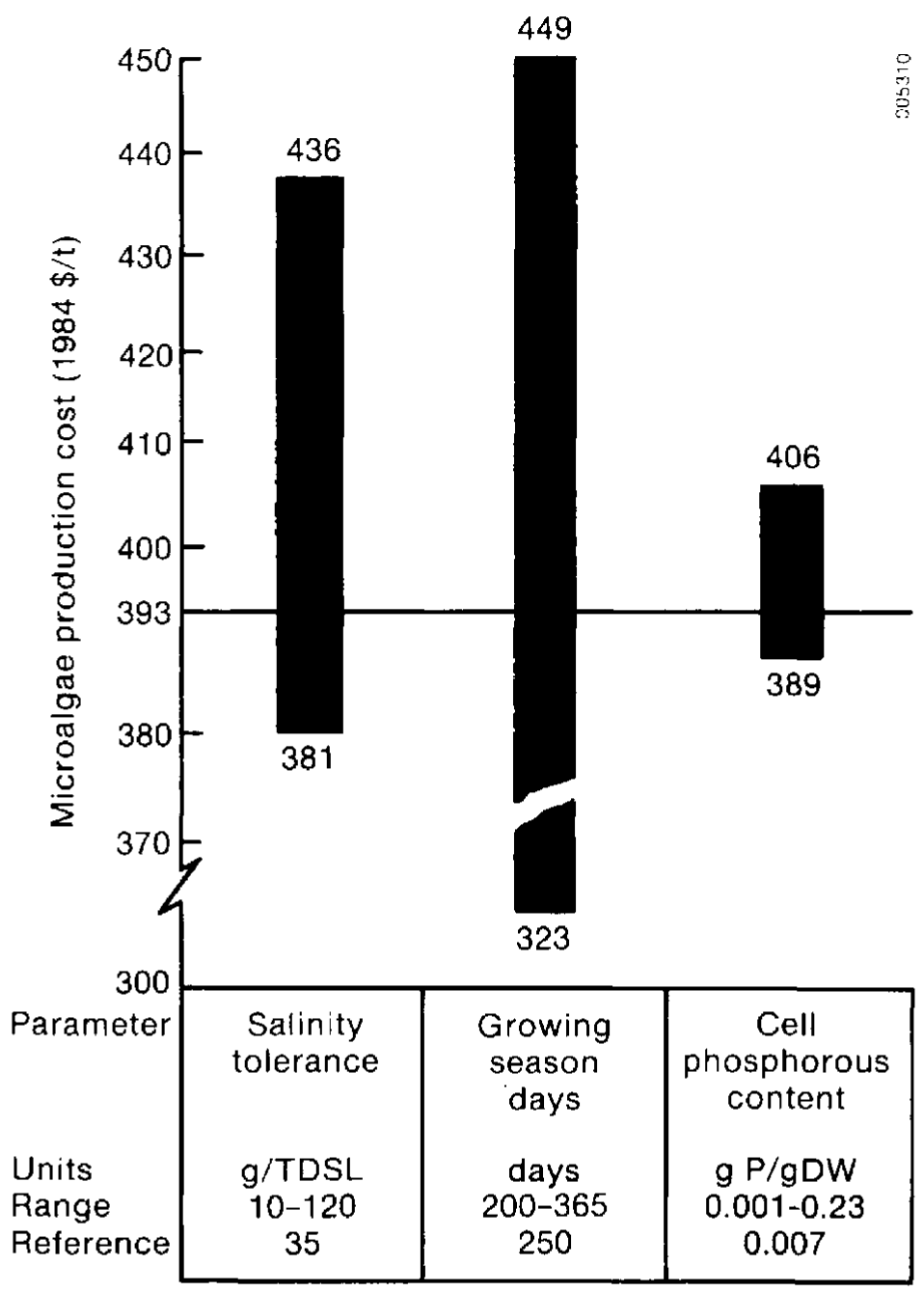

Figure 5-3. Biological Parameter Sensitivities

increasing the price by about $63 \%$. We illustrate these impacts for completeness as there is no reason to believe that real increases in microalgae costs will exceed those of the general economy.

The reference case assumes an open system design with culture exposed to the environment. Covers have been proposed as a means to protect the culture from adverse environmental impacts. Tq see how the addition of covers affects costs, we add capital costs equivalent to $\$ 1.50 / \mathrm{m}^{2}$ for the effective culture area. The resulting feedstock cost is $\$ 490 / t$, a $22 \%$ increase over the reference case. We did not reflect the possible productivity benefits of covers in this analysis in the spirit of the sensitivity analyses of this section. Below, we will examine the integrated impact of covers.

The reference cost assumes a capital cost of $\$ 5000 /$ ha for pond liners. To capture the uncertainty attendant to this particular component we examine a $25 \%$ decrease and increase in cost. As illustrated in Figure 5-5, the variation assumed changes algal cost by only $2 \%$, an encouraging result given the speculation that current liner technology may not be sufficient for extended, commercial operation (Dodd 1984). 


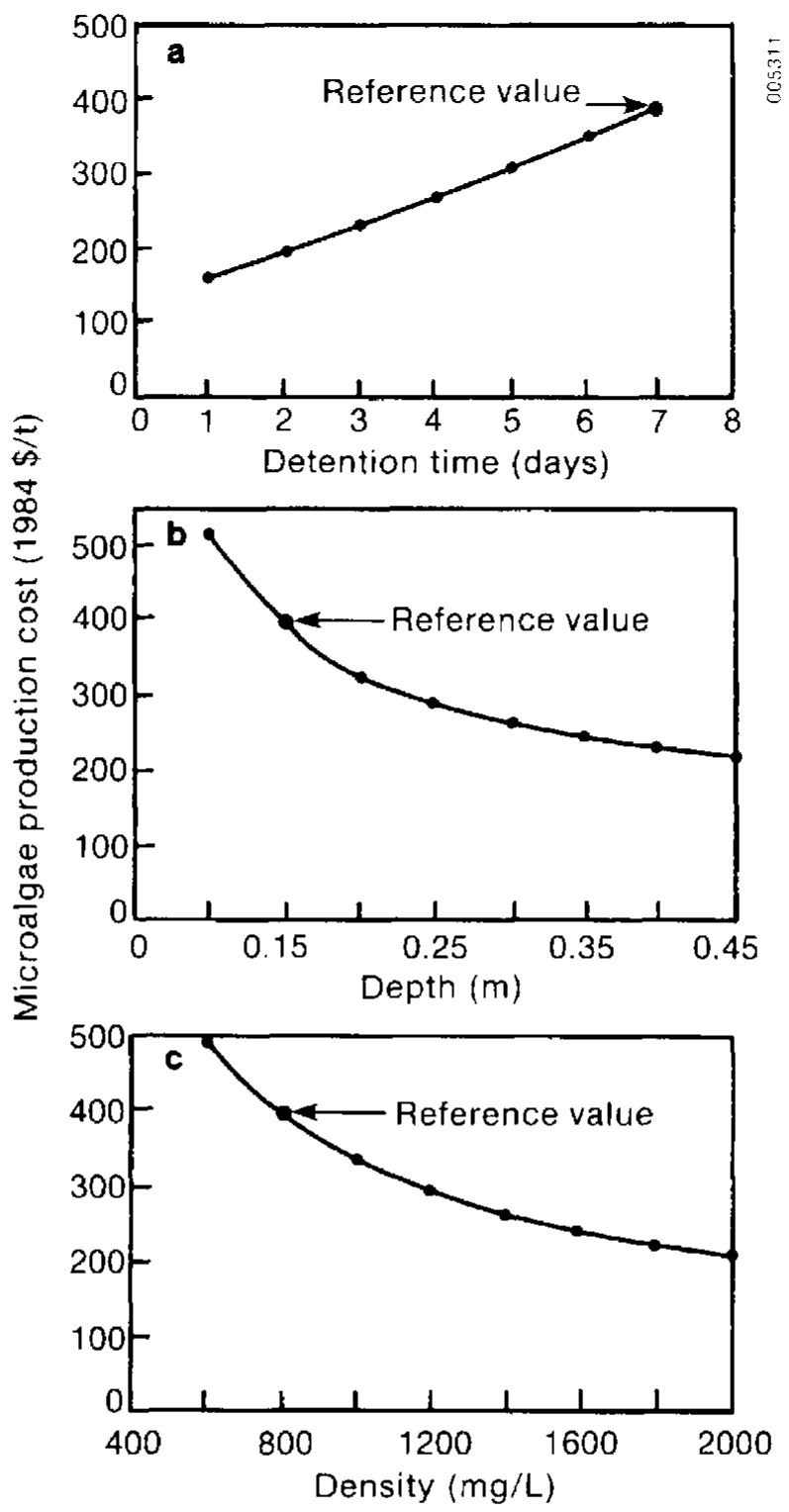

Figure 5-4. Sensitivity of Algal Production Cost to Detention Time, Depth, and Density

We reflect capital cost uncertainty by increasing them $25 \%$, a means of accounting for possible underestimation of balance of system costs. The result is an $8 \%$ increase over the reference algal production cost.

Finally, higher rates of return are of ten applied to new, risky investments for compression with more mature technologies. We raise the return on debt and common and preferred stock by $50 \%$ over the reference case and find that production cost increases by $8 \%$ to $\$ 425 / \mathrm{t}$. This increase may be appropriate for early, risky applications but a mature microalgae technology should compete for capital at rates closer to the reference values.

In the next section we look at integrated representations of these uncertainties as a means of bounding expected costs and of deriving the extreme bounds of required technology improvements. 


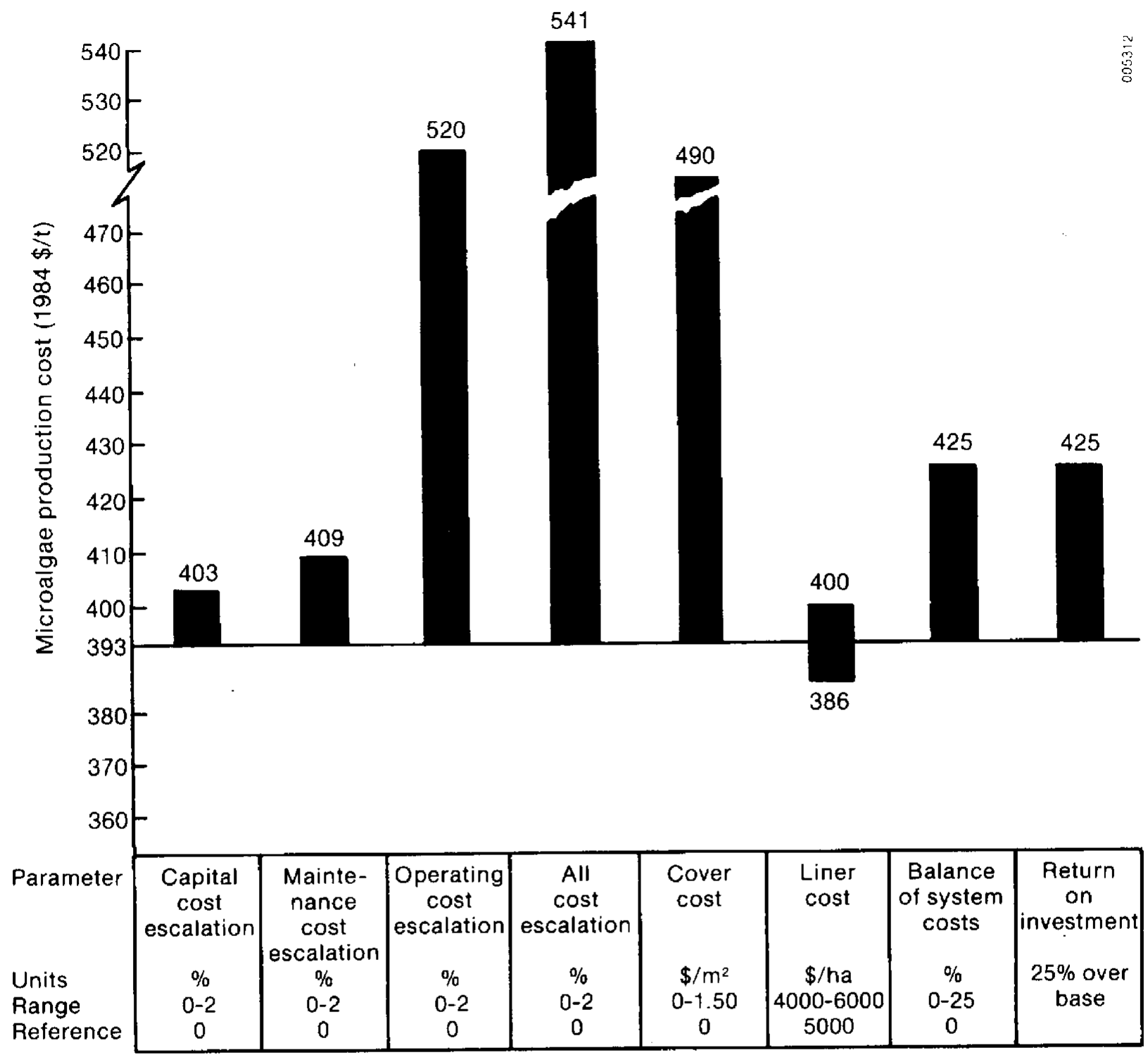

Figure 5-5. Financial Parameter Sensitivities

\subsection{Important Parameters for Further Evaluation}

Figures 5-1 through 5-5 present all the sensitivity results of changing input assumptions to calculate product cost. Table 5-1 lists those parameters by category that have a significant effect on feedstock cost through the range of assumed values. Analyzing sensitivities performed one at a time gives an indication of the relative importance of the parameters. However, structural linkages among variables, when taken into account, can exacerbate or dampen single variable sensitivities. The next section examines functional linkages and their effect on microalgal production cost with the object of identifying technology configurations that maintain resource and biological feasibility and marketdetermined production cost goals. 
Table 5-1. Summary of Most Critical Resource, Facility Design, and Biology Parameters

Resource

Evaporation

Source water salinity

Carbon content of source water

Energy cost

Water supply costs

Distance to carbon dioxide supply

$\underline{\text { Facility Design }}$

Effective culture area

Downtime

Channel width

Mixing velocity

Culture depth

Carbon outgassing losses

Nitrogen outgassing losses

Mixing drive efficiency

Harvester solids removal efficiency

Biology

Salinity tolerance

Phosophorus content

Growing season

Algal chemical content

Algal productivity 


\subsection{EVALUATION OF IMPORTANT TECHNOLOGY ISSUES}

In this section we assess the major $R \& D$ issues associated with producing fuels from microalgae in order to determine the viability of the technology and the appropriate development paths. This objective is achieved in five sequential stages of analysis. The first is to determine the important linkages among the technical parameters that ultimately dictate the cost of producing microalgae feedstocks.

Section 5.0 discussed 20 critical parameters in the mass culture system identified through sensitivity analysis. These parameters are too numerous for extensive comparative static analysis and are related in important respects. By establishing the linkages among them, the analysis in Section 6.1 develops quantifiable cost impacts, brings the major research issues to the forefront, and thereby facilitates the analysis of $R \& D$ priorities.

The second stage of the analysis is to evaluate alternative fuel products to determine which are the best candidates for microalgae production. This stage is conducted by a process of elimination that includes three distinct steps. (1) The total revenues are projected for each fuel product and production configuration, including the value of the primary fuel and by-products produced; (2) the costs of fuel processing for the reference case are subtracted from the estimated revenues, and the residual is the amount that can be spent for the feedstock, which we call allowable feedstock processing costs; and (3) allowable feedstock processing costs are compared to projected feedstock production costs for the five major fuel types. Those fuel products for which projected production costs are closest to the allowable processing costs are judged to be the best candidates for production from microalgae. These fuel cycles serve as the basis for defining research targets that, if realized, meet the economic requirement--a commercially viable fuel production technology.

The third stage of analysis is to construct and compare attainability targets for microalgae feedstocks production. Our comparison of costs includes a number of assumptions about critical technical parameters, such as microalgae productivity. This analysis is the basis for establishing attainability targets by varying those parameters that can be affected by $R \& D$ investments.

The fourth stage of the analysis is to evaluate attainability targets for fuel processing. These attainability targets embody improvements in specific parameters that are judged to be technically attainable but require research and development. Attainability targets for feedstock production and fuel processing are combined to construct an integrated attainability concept, which is compared with fuel cost goals to determine the potential of the technology for becoming a cost-competitive source of energy. Finally, the targets are then subjected to sensitivity analyses to examine alternative combinations of parameter levels that meet the cost-effectiveness test. This last step explores alternative $R \& D$ paths that fulfill the criteria for technology feasibility.

The objective of this analysis is to ascertain what improvements in microalgae production and processing technology are needed for competitive (with conventional fuel technology) fuel production. In order to judge what level of parameter improvements are needed, we need some standard or goal to serve as the threshold for costcompetitiveness. We have chosen the year 2010 cost goals for microalgae-derived fuels presented in Section 4.0 (and summarized in Table 6-1). We wish to derive, if feasible, an integrated set of improvements in microalgal production and fuel processing that achieve 
fuel costs equal to or less than these cost goals. Our method of analysis therefore calculates fuel and by-product revenues at their respective cost goal levels, and the standard by which we measure technology feasibility is the comparison of feedstock production cost with the allowable processing cost. Equality of these values signals fuel production at the target cost goal level since the sum of value added at each stage of production exactly matches the competitively determined market price.

Before identifying and evaluating technology improvements, it is essential to understand the implications of the methodology. Fixing fuel values at their cost goals, and evaluating the technology by equating feedstock values in the two stages of production, ignores uncertainty inherent in the projection of year 2010 fuel values. This uncertainty is not passed through to the evaluation of production and processing costs, which are compared as though they are discrete, certain indicators of costs, values, and technology feasibility.

Initially, we make rigid (deterministic) comparisons based on these cost goals since they represent a uniform estimate of year 2010 values and since the present goal of the microalgae program is to achieve cost effectiveness soon after the turn of the century. But neither the cost goal values nor their year should be taken as fixed targets that, if not met, signal technology infeasibility. Fuel values could be higher in 2010, so exact equivalence of microalgae-based fuels with forecasts of conventional fuels is not an absolute indicator. Moreover, expected depletion of fossil fuel reserves results in everincreasing fuel prices after 2010, so if the target is missed for that year, the technology may still be able to compete at a later time. We seek to match the conditions of technical feasibility to market conditions to aid in program development decisions.

The comparisons in the following sections set very rigid criteria for technology feasibility--goals for year 2010 must be met exactly or exceeded. We will relax this constraint after it has served its purpose, which is to focus our attention toward identifying specific, attainable research and development improvements. Sensitivity analyses in the final sections will provide a wider perspective on the questions of technology feasibility and $R \& D$ requirements.

\subsection{Important Linkages and Critical Issues in Microalgae Feedstock Production}

The parameter screening in Section 5.0 evaluated the changes in algal biomass production costs resulting from a change in the value of an individual parameter, all other parameters held at their reference level. This procedure isolates the relative importance of parameters but ignores biological, engineering, and economic interrelationships inherent in the technology configuration.

For example, we saw that varying culture depth from the reference value has a significant impact on the cost of producing lipids (see Section 5.0). However, this first-order impact, estimated by varying a single parameter, could be reduced or exacerbated when secondary impacts are taken into account. For example, at constant productivity, the advantage of deeper cultures is that there is less variation in culture conditions (e.g., temperature) with diurnal variation. Such protection may be very important to microalgae that meet high performance levels but are sensitive to temperature. On the other hand, deeper cultures increase head loss and flow rates in the system, resulting in higher energy consumption for mixing and pumping. In order to map response effects accurately and use these mappings to determine the optimal $R \& D$ approach, we must explore these 
Table 6-1. Major R\&D Issues for Fuels from Microalgae

\begin{tabular}{|c|c|}
\hline & $\begin{array}{l}\text { Water demand } \\
\text { Carbon dioxide demand } \\
\text { Algal productivity and products } \\
\text { Harvesting }\end{array}$ \\
\hline A. & $\begin{array}{l}\text { Algal Production } \\
\text { - Water Demand } \\
\text { - Carbon Dioxide Demand } \\
\text { - Algal Productivity and Products } \\
\text { - Algal Harvesting }\end{array}$ \\
\hline B. & $\begin{array}{l}\text { Fuel Processing } \\
\text { - Alternative Fuel Products } \\
\text { - Scale Economies } \\
\text { - Process Productivity } \\
\text { - By-Product Values }\end{array}$ \\
\hline C. & $\begin{array}{ll}\text { Cost Goals (1985 \$) for Fuels from Microalgae (2010) } \\
\text { - Gasoline } & \$ 1.75 / \mathrm{gal} \\
\text { - PVO } & \$ 1.75 / \mathrm{gal} \\
\text { - Ester Fuel } & \$ 1.75 / \mathrm{gal} \\
\text { - Methane } & \$ 7.40 / \mathrm{mscf} \\
\text { - Ethanol } & \$ 1.20 / \mathrm{gal}\end{array}$ \\
\hline
\end{tabular}

interdependencies; we must estimate both the marginal costs and the marginal benefits implied by variations in the system configuration or its parameters.

The reference case represents a set of equilibrated parameter levels picked so that the complete production system is a technically feasible and consistent representation of values across all components of the system. The cascading effects that emanate from the variation of a single parameter have an impact on many important process parameters. These linkages are vital also to our effort to isolate factors important in technology improvement and to evaluate ways to reduce production costs.

The initial screening of individual parameters indicate how the system parameters affect feedstock cost. However, by careful examination of these initial parameters, we can define interrelationships or linkages among groups of these parameters that enabled us to reduce the number of major effects to those listed in Table 6-1. The analysis begins with the parameter sensitivity identified in the initial screening, works through the major linkages inherent in the model, and then identifies the implications for $R \& D$ improvements. This approach can be viewed as an attempt to develop a frame of reference for evaluating alternative approaches to lower production costs by reducing a large number issues to their lowest common denominator--the technology improvements required for an economic production system. We begin by exploring interrelations with and among the algal production groupings of Table 6-1. 


\subsubsection{Water Demand}

Inspection of the sensitivities for resource and biology parameters in Section 5.0 indicates the critical sensilivity of reference production cost to water demand. In order to systematically evaluate the technology of growing microalgae in the saline waters of the southwestern United States, we begin by illustrating the linkages among water quality, environmental conditions, and algal species characteristics. Figure 6-1 illustrates the progressive linkage of source water through system design alternatives to net water demand and cost. This figure shows an expansion of the original production concept (on the right side of the figure) to include a means of mitigating high water costs--an environmental protection system or cover to reduce evaporation and nutrient outgassing (on the left side of the figure). First, we examine mechanisms for controlling water costs in the reference (uncovered) system and then we estimate the value of covers to the system.

For uncovered systems, water demand is a function of net makeup water, which is determined by evaporation; salinity of the source water; and the maximum operating salinity of the culture system, which is determined in turn by the salinity tolerance of the microalgae. For a given evaporation rate, higher source water salinities or lower species salinity tolerance result in higher blowdown requirements to maintain culture salinity. These parameters are linked; controllable parameters (e.g., species salinity tolerance) can be varied to mitigate effects of uncontrollable parameters (e.g., salinity of the source water) to minimize overall water demand, subject to the degree of control that can be exercised.

Variations of the reference cost of producing algae to variations in both the evaporation rate and the algal salinity tolerance are illustrated in Figure 6-2. The three curves, A, B, and $C$, map combinations of salinity tolerance and algae production costs for the reference, high, and low evaporation rates, respectively. Because of the effect on blowdown and water demand for any given evaporation rate, algal salinity tolerances above the reference level of $35 \mathrm{~g}$ TDS/L decrease production cost and tolerances below $35 \mathrm{~g}$ TDS/L increase costs.

The combined effect of salinity tolerance and evaporation rate, illustrated in Figure 6-2, demonstrates the importance of choosing an algal species with as high a salinity tolerance as possible to control costs when evaporation rates are high (either at a specific site or as a result of diurnal or seasonal effects). Curve $A$ shows the reference case variation in production cost due to variations in the salinity tolerance. Production costs rise, as salinity tolerance decreases, from the reference tolerance of $35 \mathrm{~g}$ TDS/L, reaching $\$ 420 / \mathrm{t}$ at $20 \mathrm{~g}$ TDS/L (Curve A, Figure 6-2). At salinity tolerances above $35 \mathrm{~g}$, (again using Curve $A$ with the reference evaporation rate) production costs decline, although slowly, until most of the cost reducing benefits have been realized at $80 \mathrm{~g} \mathrm{TDS} / \mathrm{L}$. Increasing salinity tolerance from 35 to $80 \mathrm{~g}$ TDS/L (130\%) amounts to a cost reduction of only about $2 \%$; incremental benefits are not symmetrical to incremental decrements measured relative to reference salinity tolerance.

As the evaporation rate is varied from the reference value of $0.0035 \mathrm{~m} / \mathrm{d}$ to the high and low levels, 0.01 and $0.002 \mathrm{~m} / \mathrm{d}$, respectively, the same relationship is apparent--some benefits of higher salinity tolerances are realized, while cost increases dramatically at lower than reference salinity tolerance levels. The result is most dramatic for the high evaporation case (Curve B) where costs increase almost $12 \%$ as the salinity tolerance is decreased from 35 to $25 \mathrm{~g}$ TDS/L. On the other hand, increasing the tolerance from 35 to $80 \mathrm{~g} \mathrm{TDS} / \mathrm{L}$ for the same evaporation rate (B) virtually compensates for the cost 


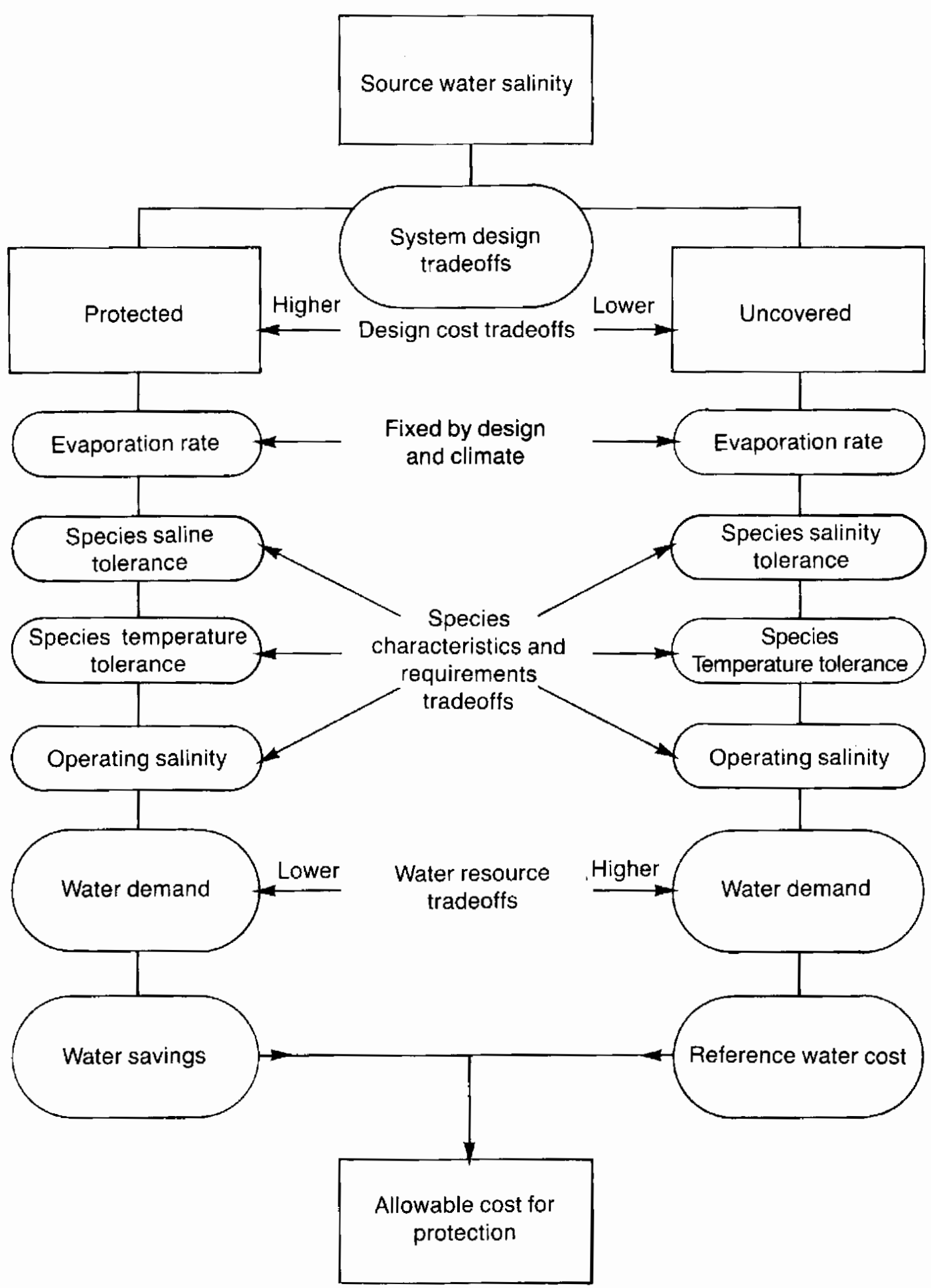

Figure 6-1. Linkages among Water Source Salinity, Water Use, and Algal Species Requirements 


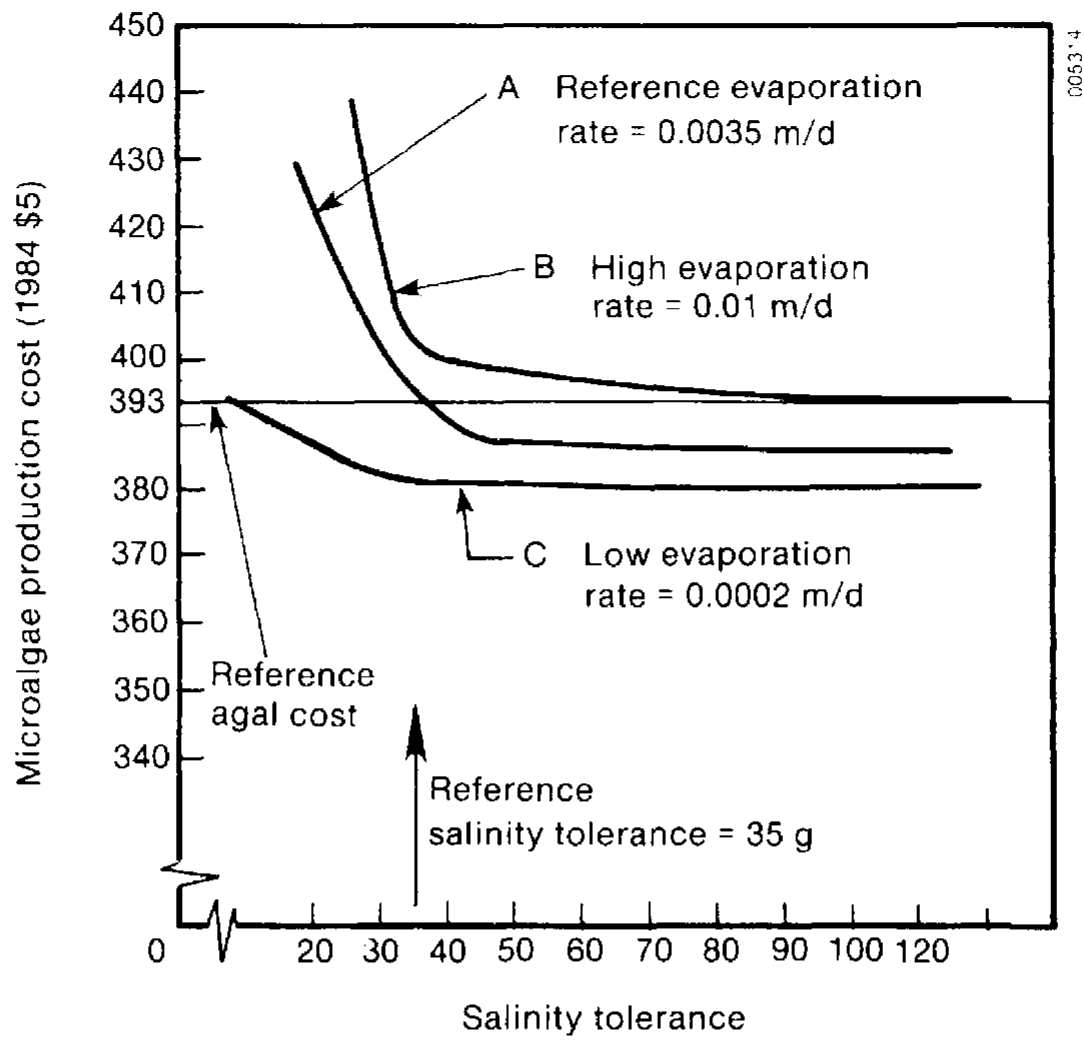

Figure 6-2. Sensitivity of Reference Production Cost to Variations in the Evaporation Rate and Algal Salinity Tolerance

increase imposed over the reference case. That is, under high evaporation situations, production costs can be maintained at the reference level $(\$ 393 / \mathrm{t})$ by raising salinity tolerance from 35 to about $80 \mathrm{~g}$ TDS/L, thus verifying a biological control function.

Uncovered algal production systems are likely to experience diurnal and seasonal changes in the evaporation rate. Thus, even if the average rate of evaporation is $0.0035 \mathrm{~m} / \mathrm{d}$, as characterized in the reference case, salinity tolerances above the reference $35 \mathrm{~g} \mathrm{TDS} / \mathrm{L}$ can help mitigate these variations. Since most benefits of increased salinity tolerance are realized at $80 \mathrm{~g}$ TDS/L, regardless of the evaporation rate, screening and selection of algal species should include provision for characterizing species that can sustain production in waters of salinity as high as $80 \mathrm{~g}$ TDS/L. This affords the maximum protection available against the effects of evaporation and provides an additional benefit in terms of mitigating the effects of higher salinity source water to be discussed below. The $80 \mathrm{~g}$ TDS/L target is the ideal, but as Figure 6-2 illustrates, there are substantial benefits to be realized by increasing salinity tolerance to the 50-60 g TDS/L level and this should serve as a minimum target for directing species selection and optimization.

Given the prominent role that evaporation rate plays in cost sensitivities, it is useful to examine strategies to reduce evaporation. Covers have been proposed as a means of providing environmental protection to algal cultures, reducing evaporation along with providing protection against dust, predators, wind, and other deleterious effects. The complete definition of the technical benefits of covers has not been established, so at this time it is not possible to define the complete system impacts. Moreover, cover systems proposed to date are not optimized for microalgae and therefore estimated costs are subject to considerable uncertainty. 
A preliminary estimate of the value of covers is derived by estimating the maximum physical impacts expected, and calculating the associated cost reduction and maximum allowable cost for covering the system, either entirely or in some intermittent fashion (only over carbon dioxide spargers, over half of the pond, etc.). Maximum benefits are calculated by lowering, from the reference to the low values, the evaporation rate and all nutrient outgassing losses; raising the growing season to 365 days; increasing the source water salinity to $35 \mathrm{~g}$ TDS/L and salinity tolerance to $80 \mathrm{~g}$ TDS/L; and evaluating the algae production cost at $\$ 0.05, \$ 0.067$, and $\$ .20 / \mathrm{m}^{3}$ water cost. We do not contend that these targets can be met singularly or collectively but use these levels only to establish the maximum expected benefits of covers.

Measured relative to the reference algae production cost of $\$ 393 / t$, the low, reference, and high water cost cases, with cover effects, result in $\$ 96 / t, 108 / \mathrm{t}$, and $112 / \mathrm{t}$ cost reductions, respectively. These savings, amortized over the facility life (20 years), translate into allowable initial capital costs for covers of $\$ 2.25-\$ 2.35$ per square meter of covering material, assuming the entire pond or raceway area is covered. Thus, covering half of the pond area doubles allowable cost and so forth, although the likelihood of the assumed optimistic parameter levels and associated cost reductions being achieved with partial coverage is low. These allowable cover costs are insufficient compared to covers typical of greenhouses that cost in the neighborhood of $\$ 15 / \mathrm{m}^{2}$ and must be replaced every 3-4 years (no allowance for maintenance or replacement is embodied in the estimated allowable cover cost). Clearly, conventional covers are not cost effective, except where production costs well in excess of $\$ 393 / \mathrm{t}$ are feasible owing to high product value. Cost-effective covers, even given high water costs, will require new materials or concepts with low costs and high benefits. Research directed in this area must look for novel concepts that can meet the rigorous standards used above and provide system protection for well below the cost of conventional covers.

Another tradeoff function, involving the development of biological improvements to mitigate resource constraints, is between algal salinity tolerance and the cost of saline water. Since algae compete with other uses for water, at least for low salinity water, we expect that the lower the source water salinity the higher the cost and therefore the greater need for higher salinity tolerance to absorb cost increases. Conversely, high salinity water has few alternative uses and should be lower in cost. Therefore, in the latter case salinity tolerance should be less important, provided the tolerance is above the salt content of the source water.

We examine this hypotheses through two cases. The first is constructed by setting the water cost at the high level $\left(\$ 0.20 / \mathrm{m}^{3}\right)$ and the corresponding source water salinity at the low value ( $10 \mathrm{~g}$ TDS/L) and then calculating the algae production cost for the reference level of salinity tolerance and for higher values (50 and $80 \mathrm{~g}$ TDS/L). The same calculations are also performed for the low water cost $\left(\$ 0.05 / \mathrm{m}^{3}\right)$ with high $(25 \mathrm{~g}$ TDS/L) source salinity. The results of these two cases (Table 6-2) confirm the hypothesis that if more expensive, lower salinity water is used, salinity tolerance of the algae is more important in terms of minimizing algal production cost.

The two cases illustrated in Table 6-2 both result in variations in price from the reference case. We can further enrich the comparison by calculating the increase or decrease in the evaporation rate that restores the cost to the reference level of $\$ 393 / \mathrm{t}$. In the high water cost case (A), the evaporation must be lower to compensate for increased water costs and for the low water case, a higher evaporation rate is allowable, thereby relaxing another binding environmental constraint. We define the equivalent evaporation rate as the level of evaporation that, given specific changes (from the reference case) in water cost and salinity, returns the algal production cost to the reference $\$ 393 / \mathrm{t}$ level. 
Table 6-2. Tradeoffs between Salinity Tolerance and Water Costs

Case A: High cost $\left(\$ 0.20 / \mathrm{m}^{3}\right) /$ low salinity $(10 \mathrm{~g}$ TDS $/ \mathrm{L})$ water

\begin{tabular}{cccc}
\hline $\begin{array}{c}\text { Salinity Tolerance } \\
(\mathrm{g} \text { TDS } / \mathrm{L})\end{array}$ & $\begin{array}{c}\text { Algae Cost } \\
(\$ / \mathrm{t})\end{array}$ & $\begin{array}{c}\text { Increase from Reference } \\
(\$ / \mathrm{t})\end{array}$ & $\begin{array}{c}\text { Equivalent } \\
\text { Evaporation Rate }\end{array}$ \\
\hline 35 & 425 & 32 & 0.0009 \\
50 & 420 & 27 & 0.0010 \\
80 & 417 & 24 & 0.0012 \\
120 & 415 & 22 & 0.0020 \\
\hline
\end{tabular}

Case B: Low cost $(\$ 0.05) /$ high salinity $(25 \mathrm{~g}$ TDS/L) water

\begin{tabular}{cccc}
\hline $\begin{array}{c}\text { Salinity Tolerance } \\
(\mathrm{g} \text { TDS/L) }\end{array}$ & $\begin{array}{c}\text { Algae Cost } \\
(\$ / \mathrm{t})\end{array}$ & $\begin{array}{c}\text { Decrease from Reference } \\
(\$ / \mathrm{t})\end{array}$ & $\begin{array}{c}\text { Equivalent } \\
\text { Evaporation Rate }\end{array}$ \\
\hline 35 & 381 & 12 & 0.0085 \\
50 & 380 & 13 & 0.0090 \\
80 & 380 & 13 & 0.0100 \\
120 & 380 & 13 & 0.0150
\end{tabular}

The equivalent evaporation rate is that which restores algal production cost to the reference level, given the indicated level of salinity tolerance.

For Case A, with high water cost, the equivalent evaporation rate, assuming a salinity tolerance of $35 \mathrm{~g}$ TDS/L, is $0.0009 \mathrm{~m} / \mathrm{d}$, a reduction of $75 \%$ compared to reference evaporation. Thus, to make up for the more expensive water, drastic reductions in evaporation rate are required to maintain algal production costs. If salinity tolerance is raised from 35 to $80 \mathrm{~g}$ TDS/L to offset the increase in water cost, the equivalent (allowable) evaporation rate in Case A rises to $0.0012 \mathrm{~m} / \mathrm{d}$--mitigating the higher water cost still requires a $65 \%$ reduction in evaporation rate or production costs will rise from the reference case level of $\$ 393 / \mathrm{t}$. These stringent evaporation rate limits are not achievable without covers in extensive technology adoption in desert climates and, as demonstrated above, are not currently feasible. Our first approximation of the impacts of high water cost on microalgae for fuel production suggests that high cost/low saline water resources are not economical and algae must be developed to withstand high source water salinities.

The low water cost case, Case B in Table 6-2, is characterized by reduced production costs owing to the assumed lower (than reference) water cost associated with the higher source water salinity. In contrast to Case $A$, the equivalent evaporation rate is higher than the reference rate of $0.0035 \mathrm{~m} / \mathrm{d}$; higher evaporation can be tolerated because the water is cheaper. At $35 \mathrm{~g}$ TDS/L salinity tolerance in Case B, the equivalent evaporation rate is over $0.0085 \mathrm{~m} / \mathrm{d}$ and higher for higher salinity tolerances. The availability of low cost water greatly relaxes the evaporation rate constraint, at least in the reference production regime. Note, however, that in Case B even though evaporation rates considerably higher than the reference case are allowable, holding production cost ${ }_{3}$ constant at the reference $\$ 393 / \mathrm{t}$, the absolute cost reduction due to low cost $\left(\$ 0.05 / \mathrm{m}^{3}\right)$ water and 
very high algal salinity tolerance $(80 \mathrm{~g} \mathrm{TDS} / \mathrm{L})$ is only $\$ 12-13 / \mathrm{t}$. Low water cost helps reduce production costs, but only marginally. To substantially reduce the reference case microalgae production cost, we will need to identify other high impact areas that offer the opportunity for cost reduction.

To summarize this section on water costs, we conclude that algae salinity tolerance is an important characteristic and that technology feasibility depends on development of species with a tolerance of at least $35 \mathrm{~g} \mathrm{TDS} / \mathrm{L}$, and the goal should be at least $50 \mathrm{~g} \mathrm{TDS} / \mathrm{L}$ to provide most of the potential protection possible against extreme environmental conditions. Although covering algal systems offers many benefits, the task of developing a cost-effective covering system is arduous. At the extreme, the allowable cost for covers in the reference system is only about $2.25 / \mathrm{m}^{2}$ of production area, well below the cost of conventional plastic covering systems. Partial covering, say over the carbon dioxide delivery system to improve utilization of this expensive input, may prove cost effective, but efforts to devise a full system cover are constrained by the low allowable cost and must be evaluated relative to other opportunities to reduce production cost.

\subsubsection{Carbon Dioxide}

The cost of carbon dioxide is a large fraction (about $30 \%$ in the reference case) of product cost because $40 \%-50 \%$ of the dry organic weight of algae is carbon. Additional carbon dioxide requirements, beyond the biological requirements for respiration and algal carbon storage, are due to carbon losses from outgassing and blowdown.

The supply assessment in Section 3.2 concluded that flue gas might be a vast potential source of large quantities of carbon dioxide for mass culture of algae. The cost of flue gas carbon dioxide is a function of three components. The first is the cost of buying the $\mathrm{CO}_{2}$ from the power plant. Given projected estimates of the demand for $\mathrm{CO}_{2}$, electric power plant operators are likely to charge a nominal price for the flue gas. The second component of $\mathrm{CO}_{2}$ price is the amortized cost of recovery, purification, and compression of $\mathrm{CO}_{2}$ from the flue gas. These recovery costs are a large part of the final delivered cost because carbon dioxide represents only about $16 \%$ of the total flue gas volume and purification requires extensive processing. The third price component is the amortized pipeline and pumping costs for delivery of $\mathrm{CO}_{2}$ to the algal production facility.

The cost $\left(\$ 0.13 / \mathrm{m}^{3}\right)$ of purchased $\mathrm{CO}_{2}$ for the reference microalgae facility was about $\$ 80 / \mathrm{t}$ microalgae produced. This cost was based on the assumptions that the microalgae system required $0.082 \times 10^{6} \mathrm{~m}^{3} / \mathrm{d}$ of carbon dioxide piped from a power plant $80 \mathrm{~km}$ from the production facility.

The effect of varying transportation distance on $\mathrm{CO}_{2}$ cost is illustrated in Figure 6-3. Unit costs of $\mathrm{CO}_{2}$, given reference demand $\left(0.082 \times 10^{6} \mathrm{~m}^{3} / \mathrm{d}\right)$ for the mass culture facility, increase $\$ 0.18$ for each addițional kilometer of pipeline. However, the unit cost of $\mathrm{CO}_{2}$ does not drop below $\$ 0.11 / \mathrm{m}^{3}$, the amortized fixed capital and operating costs of the recovery system.

Due to assumed scale economies in the transportation of carbon dioxide, the unit cost increases sharply with $\mathrm{CO}_{2}$ demand levels below $0.082 \times 10^{6} \mathrm{~m}^{3} / \mathrm{d}$. Figure $6-4$ illustrates scale economies for constructing a $\mathrm{CO}_{2}$ supply system. At $\mathrm{CO}_{2}$ delivery rates below $0.082 \times 10^{6} \mathrm{~m}^{3} / \mathrm{d}$, unit costs for carbon dioxide increase rapidly $(\mathrm{e} . \mathrm{g}$. , assuming a fixed supply distance of $80 \mathrm{~km}$ ). At delivery rates above $0.082 \times 10^{6} \mathrm{~m}^{3} / \mathrm{d}$, these unit costs 


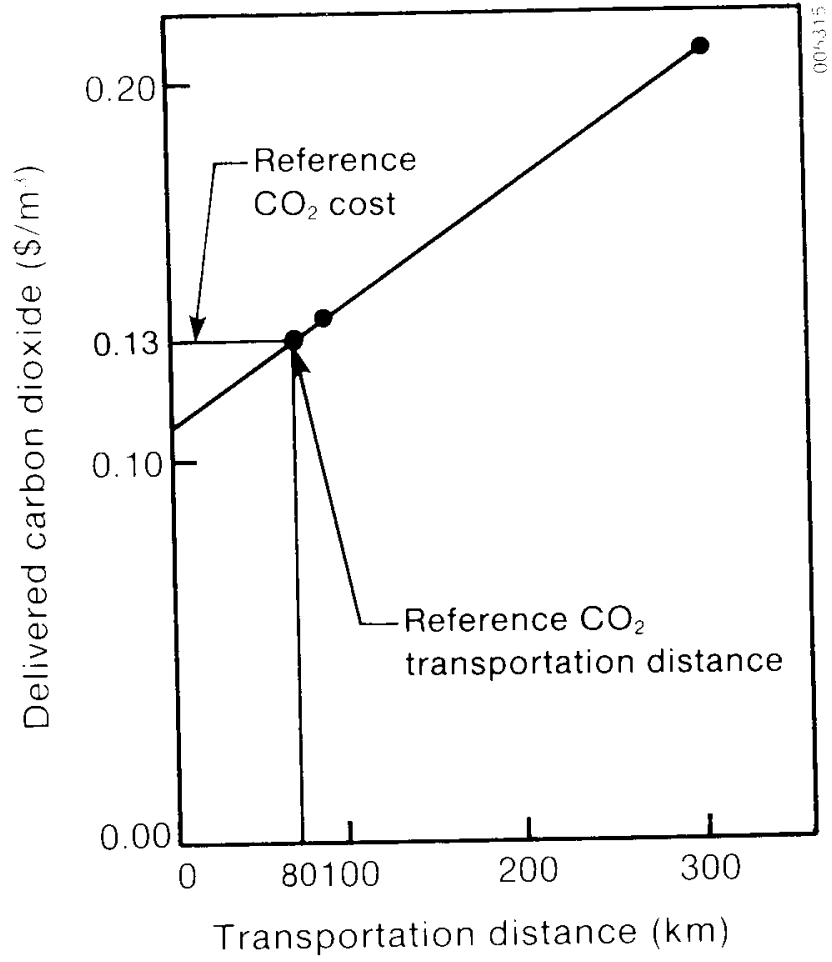

Figure 6-3. Carbon Dipxide Costs and Transportation Distance for Reference $\left(0.84 \times 10^{6} \mathrm{~m}^{3} / \mathrm{d}\right)$

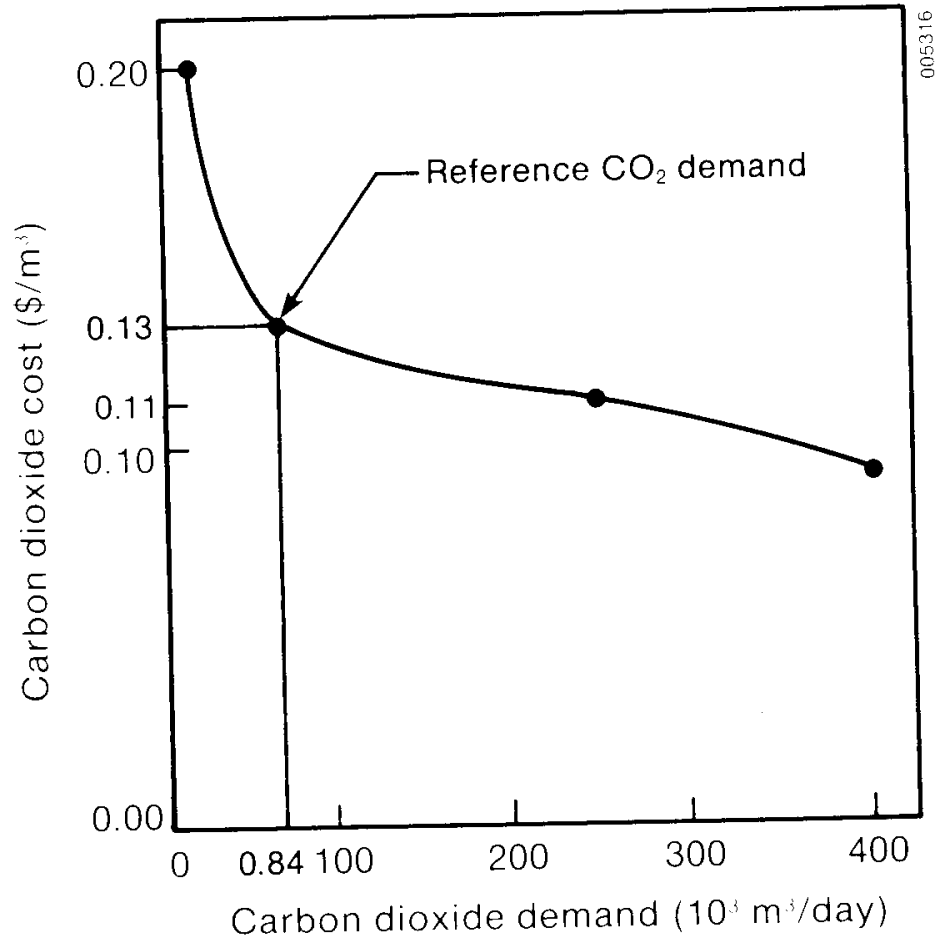

Figure 6-4. Carbon Dioxide Scale Economies for Reference Transportation Distance $(80 \mathrm{~km})$ 
decrease. If $\mathrm{CO}_{2}$ must be piped to production facilities, cost reductions can arise from the colocation of several facilities. If three facilities were colocated, the cost of $\mathrm{CO}_{2}$ would be $\$ 0.11 / \mathrm{m}^{3}$, the same result achieved above if the transportation distance is zero (the production plant is colocated with the $\mathrm{CO}_{2}$ source).

The following scenarios illustrate possible means to reduce $\mathrm{CO}_{2}$ costs to a mass culture facility. The scenarios take advantage of ways to minimize $\mathrm{CO}_{2}$ supply distance and maximize $\mathrm{CO}_{2}$ supply economies to reduce the final $\mathrm{CO}_{2}$ unit costs and therefore product cost. Table 6-3 presents the cost summary for reference during the following discussion of combining possible cost reductions to achieve minimum-cost $\mathrm{CO}_{2}$.

Above we illustrated that a $15 \%\left(\$ 0.13\right.$ to $\left.\$ 0.11 / \mathrm{m}^{3}\right)$ decrease in unit cost for $\mathrm{CO}_{2}$ is achieved if we assume that the microalgae mass culture facility is located next to a power plant and the transport distance is zero. The same result achieves by assuming that at least 3 algal facilities are colocated and scale economies are thereby realizes. The combined effect of both assumptions is a reduction in $\mathrm{CO}_{2}$ cost to $\$ 0.09 / \mathrm{m}^{3}$. Locating a mass culture facility next to a small coal- or gas-fired power plant has considerable merit, given that the mass culture facility would represent a captive market for the power plant's flue gas, some of the electricity, and the waste heat. An alternative scenario would be that the operators of the algal facility elect to construct and operate a power plant as a co-generating facility, selling excess energy to the utility grid.

A coal-fired power plant is estimated to produce $5400 \mathrm{~m}^{3}$ of flue gas per MWh containing $16 \% \mathrm{CO}_{2}$ (Hesketh 1979). A natural-gas-fired plant produces $4700 \mathrm{~m}^{3}$ of $11 \% \mathrm{CO}_{2}$ flue gas. To produce sufficient $\mathrm{CO}_{2}$ to meet a 1000-ha mass culture facility's need would require a coal-fired power plant of $5.6 \mathrm{MW}$ or a gas-fired plant of $9.4 \mathrm{MW}$. The power plant is assumed to operate at a $70 \%$ capacity. Additional power plant capacity would be required to ensure a constant, reliable source of $\mathrm{CO}_{2}$ to the facility, so two $10-\mathrm{MW}$ are used as a conservative basis for analysis of colocation of a single production facility and associated electrical power plant.

Projected capital costs of coal-fired power plants range from $\$ 900$ to $\$ 1000 / \mathrm{kW}$ with a busbar energy cost of between 30 and $40 \mathrm{mils} / \mathrm{kWh}$, depending on the type of coal and region of the country (Reynolds 1982). Because of the small size of the coal plants, a capital cost of $\$ 1200 / \mathrm{kW}$ was assumed. The two $10-\mathrm{MW}$ power plants would add $\$ 35$ million to the algal plant capital costs. Operating costs for the power plant are assumed to be recoverable through the sale of excess energy ( $95 \%$ of plant output) to the utility grid, sale of energy (at $\$ 0.03 / \mathrm{kWh}$ ), and sale of carbon dioxide to the mass culture facility. These transactions, accomplished through "arms-distant" agreements for internal accounting purposes, would ensure a constant supply of $\mathrm{CO}_{2}$ and energy to the facility. The resulting $\mathrm{CO}_{2}$ cost is $\$ .07 / \mathrm{m}^{3}$ and algae production costs drop to $\$ 356 / \mathrm{t}$ (from the reference $\$ 393 / t$ ).

If several microalgae production systems are colocated with a large coal facility, then scale economies are possible due to economies in power plant production rather than $\mathrm{CO}_{2}$ transportation. Five colocated production facilities combined with ownership of a $100-\mathrm{MW}$ coal-fired plant might be able to internally price $\mathrm{CO}_{2}$ as low as $\$ 0.05 / \mathrm{m}^{3}$ and lower production costs to $\$ 345 / \mathrm{t}$.

Above, we illustrated specific scenarios that lower the cost of $\mathrm{CO}_{2}$ from the reference level of $\$ 0.13 / \mathrm{m}^{3}$ to as low as $\$ 0.05 / \mathrm{m}^{3}$, thereby lowering production costs to as low as $\$ 345 / \mathrm{t}$. Over the range of $\mathrm{CO}_{2}$ costs from zero to $\$ 0.20 / \mathrm{m}^{3}$, a $1 \phi / \mathrm{m}^{3}$ change in $\mathrm{CO}_{2}$ cost 
Table 6-3. Summary of Alternative Scenarios to Reduce $\mathrm{CO}_{2}$ Costs to a Mass Culture Facility

\begin{tabular}{|c|c|c|}
\hline Scenario & ${ }_{\left(\$ 9 \mathrm{~m}^{3}\right)}^{\mathrm{CO}_{3}}$ & $\begin{array}{l}\text { Algae Production } \\
\qquad(\$ / t)\end{array}$ \\
\hline Reference system & 0.13 & 393 \\
\hline $\begin{array}{l}\text { Power plant ownership } \\
\text { increase scale }\end{array}$ & 0.11 & 383 \\
\hline $\begin{array}{l}\text { Increase scale and colocated } \\
\text { plants } \\
\text { with olant ownershin and }\end{array}$ & 0.09 & 370 \\
\hline $\begin{array}{l}\text { With plant ownership and } \\
\text { colocated power plant } \\
\text { Several colocated power plants }\end{array}$ & $\begin{array}{l}0.07 \\
0.05\end{array}$ & $\begin{array}{l}356 \\
345\end{array}$ \\
\hline
\end{tabular}

changes algal production costs about $\$ 6 / \mathrm{t}$. So, free $\mathrm{CO}_{23}$ yields a production cost of about $\$ 315 / \mathrm{t}$ and the most expensive $\mathrm{CO}_{2}$ considered $\left(\$ 0.20 / \mathrm{m}^{3}\right)$ implies a cost of $\$ 435 / \mathrm{t}$.

Currently, no developed and available carbon dioxide supplies, sufficient to meet a single plant, exist in the desert southwest except at sites located next to existing pipelines, and no arrangements to draw from these sources are currently in place. The reference $\mathrm{CO}_{2}$ cost of $\$ 0.13 / \mathrm{m}^{3}$ was derived by assuming the plant is located $80 \mathrm{~km}$ from a dedicated $\mathrm{CO}_{2}$ well--the cost includes provision for drilling, laying the pipe, and transporting the $\mathrm{CO}_{2}$ to the algae production site.

Isolated $\mathrm{CO}_{2}$ resources may be available to support the reference scenario but extensive algal culturing will require a supply infrastructure including vast $\mathrm{CO}_{2}$ resources, main transportation pipelines, and a distribution system to serve individual sites. If the source is a large or several large power plants colocated in the same region with the algal farms, then costs as low or lower than $\$ 0.05 / \mathrm{m}^{3}$ are possible. Alternatively, if the $\mathrm{CO}_{2}$ resources and infrastructure are developed as part of enhanced oil recovery technology, then the emergence of an algal industry may be supported by a large, reliable $\mathrm{CO}_{2}$ supply and infrastructure and low-cost $\mathrm{CO}_{2}$ will be available.

At this time, we can only speculate about the availability and cost of $\mathrm{CO}_{2}$ for microalgae production in the desert southwest. The technology development effort must emphasize the efficient utilization of $\mathrm{CO}_{2}$ through well-designed sparging systems, hydraulics and process controls, and by screening for algae that operate as close as possible to the limits of energy conversion efficiency. But even with these provisions, the cost of carbon will still dominate production costs, and factors beyond the control of the scientific domain will play a major role in the economic feasibility of funds from microalgae. Resource studies, conducted in support of the technology development program, must continually evaluate systems for providing $\mathrm{CO}_{2}$, including possibilities such as direct extraction of carbon dioxide from the atmosphere, and provide updated costs. In this way, it is possible to update targets for controllable parameters in light of the best available economic information and evaluate the feasibility of microalgae technology for fuel production. Achieving the highest possible productivity with respect to the most expensive resource is essential in all competitive production practices, and for algal production, carbon dioxide is the vital resource. 


\subsubsection{Algal Productivity and Products}

Section 5.0 presented the reference microalgae production cost of $\$ 393 / \mathrm{t}$ given a total biomass productivity of $17 \mathrm{~g} / \mathrm{m}^{2} / \mathrm{d}$ consisting of $30 \mathrm{wt} \%$ lipids. The sensitivity analysis in Section 5.0 indicates that lipid costs are highly sensitive to biomass yield and lipid content. Biomass productivity rates in excess of $40 \mathrm{~g} / \mathrm{m}^{2} / \mathrm{d}$, achieved in highly controlled, outdoor experimental cultures, and lipid yields of over $60 \%$ in laboratory exper ments indicate the potential for improving productivity or lipid content or, albeit more difficult, both.

While these experimental performance levels are encouraging, they do not by themselves establish that needed productivity improvements can be achieved in large-scale facilities on a sustained basis. The improvements in lipid content or productivity have resulted from using different species or by maximizing one characteristic, of ten at the expense of other characteristics. To examine the effect of species improvement on biomass and lipid production costs, we examine alternative productivity yields where we assume that a species is developed especially to maximize the yield of a specific product.

The algal production model (Hill 1984) used in this analysis includes as inputs culture depth, density and detention time, cell energy content (as a function of lipid, carbohydrate, protein, and ash content), and daily solar constant. Productivity and photosynthetic efficiency are then calculated. In effect, we are approximating the effect of manipulating carbon allocation in the algal cell, where lipids or carbohydrates are maximized. The purpose of this analysis is to evaluate the benefits attributable to reductions in biomass and lipid production costs from improvements in both algal productivity (or photosynthetic efficiency) and carbon allocation properties.

For our first level evaluation of the effect of productivity and algal lipid content on production cost, we examine 9 different combinations of photosynthetic efficiency and lipid content levels. Table 6-4 is a complete list of input parameter assumptions, and Figure 6-5 displays the experimental design conducted to evaluate algal species improvements. Note in Table 6-4 that, for a given photosynthetic efficiency, increasing the percentage of lipids by altering the carbon allocation comes at the expense of carbohydrate production and vice versa.

The data in Table 6-4 illustrate the impact of alternative levels of lipid content at the reference and two higher nominal levels of PSE, as illustrated in Figure 6-5. The PSE is not varied directly but is calculated through the equation

$$
\text { PSE }=\frac{\text { Depth } \times \text { Density }}{\text { Detention Time }} \times \sum_{i=1} \text { PC }_{i} E_{i} \times \text { DSC } \times 0.42
$$

where

$\mathrm{PC}_{\mathrm{i}}=$ percentage content of product $\mathrm{i}$,

$E C_{i}=$ energy content of product $i$,

$i=$ lipid, carbohydrate, protein, ash and intermediate cell products, and

DSC = daily solar constant (multiplied by 0.42 to convert PSE to a PAR basis).

According to Eq. 6-1, for any given level of PSE we can vary the lipid content, adjusting the other cell components so that the fractions add to one; algal output will remain the same but PSE will vary as will production costs. So, a higher lipid content, offset by a lower carbohydrate, for fixed productivity, requires more energy inputs $\left(\mathrm{CO}_{2}\right.$, etc. $)$. For variations about the reference case of $4.86 \mathrm{PSE}$, we simply adjust the relative cell product contents to evaluate different lipid contents (20\% and $50 \%)$. 
Facility size ha Module size ha
Photosynthetic efficiency PAR \%

\section{Lipid} content

$\%$

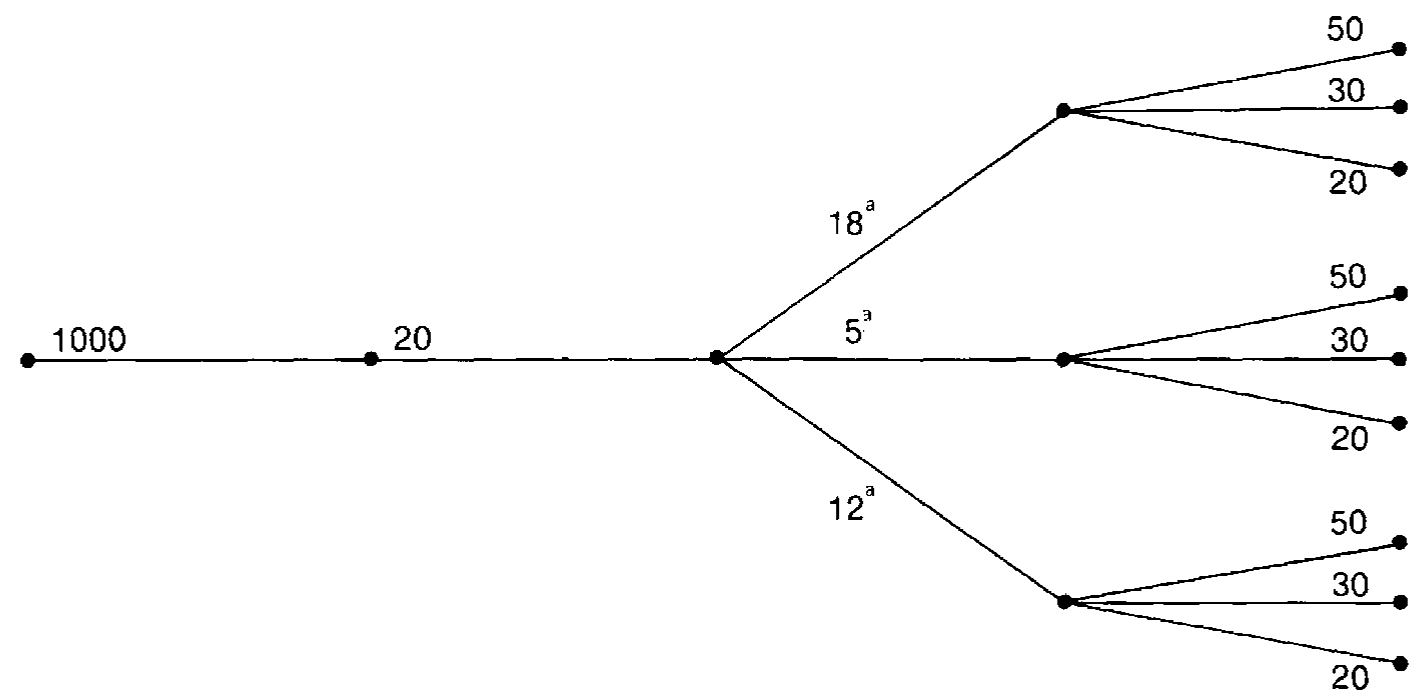

${ }^{a}$ Nominal PSE. Actual PSE level is system determined for each set of assumptions.

Figure 6-5. Experimental Design for Evaluation of Algal Species Improvements

To evaluate the changes in lipid content at other nominal rates of PSE, e.g., 12\% and $18 \%$, it is necessary to calibrate all elements of Eq. 6-1 such that the nominal PSE is achieved. This is achieved by setting the lipid content at the reference level of $30 \%$ and varying depth, density, and detention time until a PSE of the nominal rate (12\% and $18 \%$ ) results and then lipid content can be varied to $20 \%$ and $50 \%$. A large number of combinations of depth, density, and detention time can be constructed such that PSE equals the nominal design levels of $12 \%$ and $18 \%$. To get the desired PSEs, we first lowered detention time to bring PSE close to the desired level and then adjusted the density to get the nominal level (within 0.1\%). Table 6-4 gives the input parameter values used to identify the level of density and detention time in Eq. 6-1 and used as the basis for altering the lipid content. Because we selected integer values for detention time and density levels were rounded to the nearest $5 \mathrm{mg} / \mathrm{L}$, the resulting PSEs do not match exactly the nominal experimental design levels indicated in Figure 6-5.

As indicated in Table $6-4$, sensitivity of lipid content is examined at three different levels of algal biomass production corresponding to the three different levels of PSE. But, for any nominal level of PSE, varying the lipid content from the reference level of $30 \%$ changes the gross lipid output and the PSE. Thus, costs of production change since variations in cell energy content change input requirements even though productivity remains constant.

The costs of producing algal biomass and algal lipids for each case identified in Table 6-4 are listed in Table 6-5 and the results are illustrated in Figure 6-6. Increasing the nominal PSE from the reference $4.86 \%$ to $12 \%$ and to $18 \%$, with lipids held constant, reduces 
Table 6-5. Comparison of Reference Algal Feedstock Values and Attainable Levels for Productivity Variations

\begin{tabular}{|c|c|c|c|c|c|}
\hline \multicolumn{3}{|c|}{ Productivity Assumptions } & \multirow[b]{2}{*}{$\begin{array}{c}\text { Major Fuel } \\
\text { Product }\end{array}$} & \multicolumn{2}{|c|}{ Comparative Values } \\
\hline $\begin{array}{l}\text { Photosynthetic } \\
\text { Efficiency } \\
\text { (\%) }\end{array}$ & $\begin{array}{l}\text { Lipid } \\
\text { Content } \\
\text { (\%) }\end{array}$ & $\begin{array}{l}\text { Biomass } \\
\text { Annual } \\
\text { Production } \\
\left(10^{3} t\right)\end{array}$ & & $\begin{array}{c}\text { Allowable } \\
\text { Feeds tock } \\
\text { Processing Cost a } \\
(\$ / t)\end{array}$ & $\begin{array}{c}\text { Productivity } \\
\text { Attainable } \\
\text { Production Cost } \\
(\$ / t)\end{array}$ \\
\hline 4.21 & 20 & 33 & $\begin{array}{l}\text { Ethanol } \\
\text { Pvo } \\
\text { Ester fuel } \\
\text { Methane } \\
\text { Gasoline }\end{array}$ & $\begin{array}{l}12 \\
63 \\
50 \\
62 \\
69\end{array}$ & 383 \\
\hline 4.86 & 30 & 33 & $\begin{array}{l}\text { Ethanol } \\
\text { PVO } \\
\text { Ester fuel } \\
\text { Methane } \\
\text { Gasoline }\end{array}$ & $\begin{array}{l}48 \\
85 \\
72 \\
83 \\
97\end{array}$ & 393 \\
\hline 5.58 & 50 & 33 & $\begin{array}{l}\text { Ethanol } \\
\text { PVo } \\
\text { Ester fuel } \\
\text { Methane } \\
\text { Gasoline }\end{array}$ & $\begin{array}{r}74 \\
109 \\
98 \\
101 \\
138\end{array}$ & 388 \\
\hline 10.36 & 20 & 82 & $\begin{array}{l}\text { Ethanol } \\
\text { PVO } \\
\text { Ester fuel } \\
\text { Methane } \\
\text { Gasoline }\end{array}$ & $\begin{array}{l}41 \\
79 \\
75 \\
76 \\
88\end{array}$ & 214 \\
\hline 11.96 & 30 & 82 & $\begin{array}{l}\text { Ethanol } \\
\text { PVO } \\
\text { Ester fuel } \\
\text { Methane } \\
\text { Gasoline }\end{array}$ & $\begin{array}{r}71 \\
102 \\
100 \\
97 \\
117\end{array}$ & 224 \\
\hline 13.73 & 50 & 82 & $\begin{array}{l}\text { Ethanol } \\
\text { Pvo } \\
\text { Ester fuel } \\
\text { Methane } \\
\text { Gasoline }\end{array}$ & $\begin{array}{r}96 \\
128 \\
132 \\
116 \\
160\end{array}$ & 217 \\
\hline 17.65 & 20 & 123 & $\begin{array}{l}\text { Ethanol } \\
\text { pvo } \\
\text { Ester fuel } \\
\text { Methane } \\
\text { Gasoline }\end{array}$ & $\begin{array}{l}50 \\
82 \\
80 \\
77 \\
91\end{array}$ & 171 \\
\hline 18.07 & 30 & 123 & $\begin{array}{l}\text { Ethanol } \\
\text { pvo } \\
\text { Ester fuel } \\
\text { Methane } \\
\text { Gasoline }\end{array}$ & $\begin{array}{r}76 \\
105 \\
106 \\
99 \\
124\end{array}$ & 180 \\
\hline 20.76 & 50 & 123 & $\begin{array}{l}\text { Ethanol } \\
\text { Pvo } \\
\text { Ester fuel } \\
\text { Methane } \\
\text { Gasoline }\end{array}$ & $\begin{array}{l}100 \\
133 \\
142 \\
118 \\
167\end{array}$ & 176 \\
\hline
\end{tabular}

${ }^{a}$ Allowable feedstock cost is the maximum value of the feedstock to the indicated processing facility in the production and sale of products and by-products at the prices dictated by the fuel costs goals. Feedstock allowable costs are calculated for the reference processing systems.

${ }^{b}$ Productivity attainable production cost is the cost of feedstock production for the indicated levels of photosynthetic efficiency, lipid content and biomass production with all other variables held at their reference level. Thus, the concept of attainability, as used here, relates only to improvements in algal productivity. 


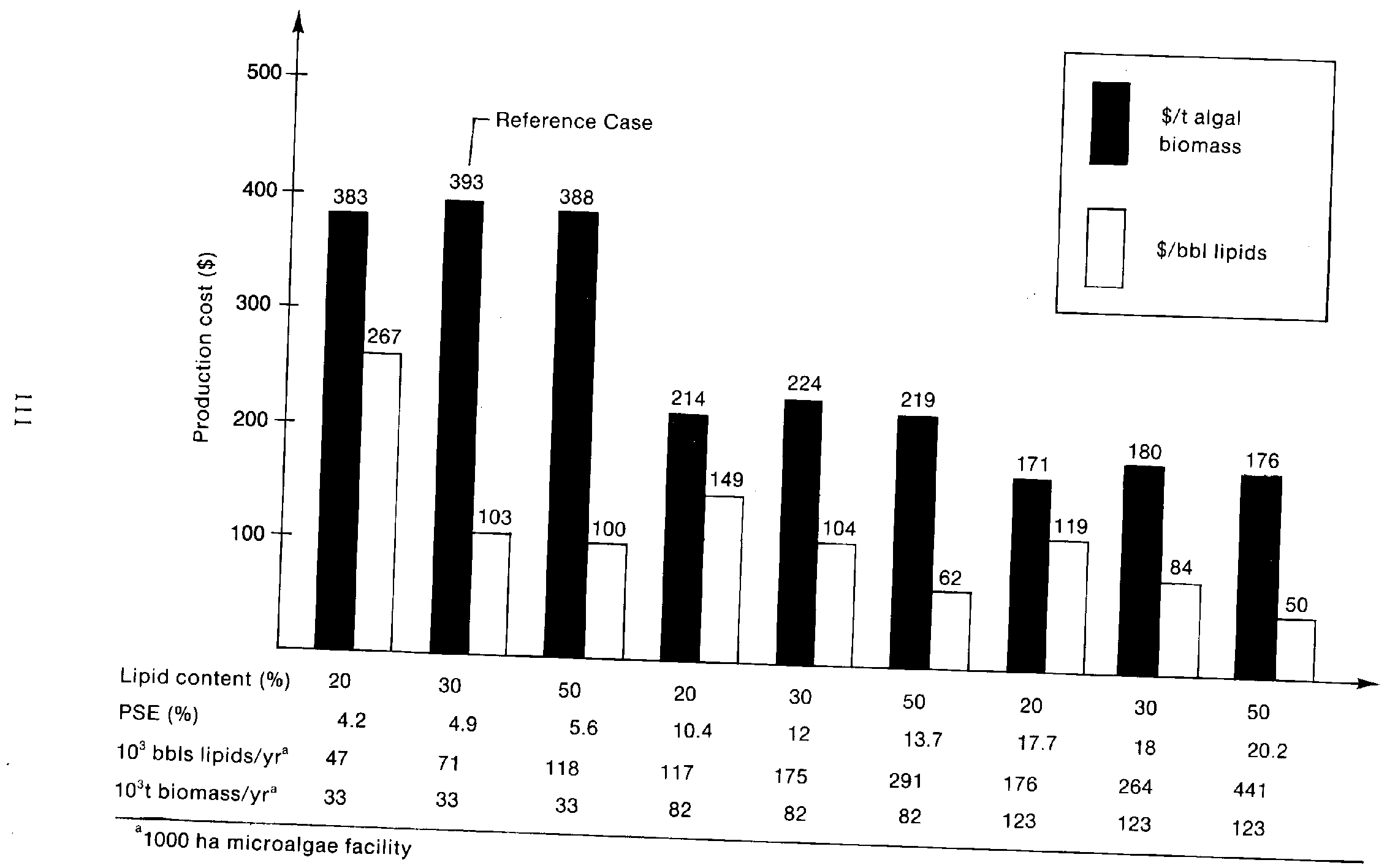

Figure 6-6. Biomass Production Cost Sensitivity to Photosynthetic Efficiency and Lipid Content 
the algal product cost from the reference $\$ 393 / \mathrm{t}$ to $\$ 224 / \mathrm{t}$ and $\$ 180 / \mathrm{t}$, reaffirming the large impact of PSE (through improvements in depth and density, characteristics of the biological culture system) on algal production cost. Lipid costs drop considerably less, only about $20 \%$ from increases of $5 \%$ to $18 \%$ PSE, since the cost is calculated based on sale of the lipids only; although lipid output increases as PSE increases, carbon costs per unit of lipid are constant, and a large proportion of operating costs therefore increase proportionately. [Thus, while lipid production increases from 71 to 264 thousand barrels from $5 \%$ to $18 \%$ PSE (lipid content constant), an increase of $271 \%$, per unit costs decrease only 50\%, so the cost per barrel drops only about 50\%.] Since we assume that variable costs increase in proportion to production, improved productivity spreads the fixed cost over a large base, but total costs do not drop proportionally with productivity improvement.

Variations in lipid content around a given PSE level illustrate that while the cost per ton of algae varies only slightly (reflecting increased or decreased inputs to support the implicit energy content of the cells), the lipid cost varies dramatically as fixed costs are spread over proportionally more or less barrels of lipid produced. Reference costs of $\$ 103$ per barrel lipids increase sharply $(60 \%)$ if only $20 \%$ lipids are produced and decrease slightly at $50 \%$ lipid content. At higher PSEs, where algal productivity is higher, the variations about $30 \%$ are more symmetric, approximately $40 \%$. Lipid costs, across both PSE and lipid content variations, range from $\$ 267 /$ barrel to $\$ 50 /$ barrel. While these data help show the impact of lipid content on the cost per barrel, evaluating the value of the algae produced requires examining refining processes and final product values, a topic addressed below. The cost comparison notwithstanding, algal productivity improvements are essential to realizing substantial decreases in algal production costs.

\subsubsection{Harvesting}

As discussed in Section 3.0, the reference harvesting system, consisting of microstrainers followed by centrifugation, contributes over $25 \%$ to the total capital investment of the mass culture facility. In Section 5.0, some variations in harvesting strategy were considered. Increasing solids removal efficiency would decrease operating costs by allowing the use of smaller equipment and thus less electric power to harvest the same amount of microalgae. Another type of first-stage harvester, the belt-filter, was also considered but was found to increase product cost despite this system's higher concentration ratio of 70 , compared with the microstrainer's concentration ratio of 10 .

There is much uncertainty concerning the technology and cost of harvesting algae. Solidliquid separation is an inexact science, especially when the important characteristics of the influent vary as widely as in microalgae species. Also, most of the available technical data were gained using fresh-water influent streams, and the effects of saline water on harvestability are difficult to estimate.

Operating strategies can be suggested that might improve the harvesting situation. Increasing culture density will decrease the overall concentration factor required by the harvesting system. The more expensive stage (usually the second stage) could then be scaled back, resulting in capital and operating cost savings. Operating at the higher density allows shallower pond depth, which decreases head losses and reduces power requirements. These savings must be weighed against the decreased temperature stability at the shallower depth systems. More operating data will be required to fully evaluate these and other harvesting strategies to determine the best solution to the harvesting problem. Data are insufficient at this time to characterize completely all harvesting research improvements and their impact on microalgae production cost. 


\subsection{Assessment of Fuel Product Options}

The first task in evaluating fuel processing options is to establish a reference case to characterize current processing technology status and to identify potential improvements. We use the basic process data and costs presented in Section 4.0 for each of the five fuel options to be evaluated. Then, we fix the value of the final fuel product, and all by-products, and derive the total revenues for each fuel product. The difference between revenues and processing costs gives an allowable feedstock processing cost--the amount a processing facility operator can afford to pay for the microalgae feedstock and produce the final product slate at the given prices. These allowable costs are compared to algal production costs for various levels of productivity to determine the feasibility of the various fuel product options. That is, algal production costs must be less than or equal to the allowable feedstock cost for the system to be judged cost effective by the fuel price standards established for 2010.

\subsubsection{Process Options}

The processing costs developed in Section 4.0 are sensitive to both the total amount of algal biomass processed and the lipid and carbohydrate content of the feedstock. Thus, in developing processing cost and subsequent allowable feedstock costs, we match assumptions used in developing feedstock production costs with those employed for processing cases: the quantity and content of the feedstock processed are matched to the corresponding productivity and feedstock composition assumptions for every combination of production and processing. In all cases, the processing plants process the output of the 1000-ha facility.

Figure 6-7 illustrates the cases evaluated for screening fuel processing options. Three levels of photosynthetic efficiency and three levels of lipid content are examined and correspond to the productivity linkages examined in Section 6.1. The five fuel options and processes correspond to those identified in Section 4.0. Thus, a total of 45 different allowable costs for fuel production are estimated and compared to the corresponding production cost, where allowable and production costs are matched according to the assumptions about photosynthetic efficiency, lipid content, and fuel type.

Throughout the next section we compare productivity (photosynthetic efficiency and lipid content) cases (which we call productivity attainable production cost) to reference fuel processing values (allowable feedstock processing costs) to screen alternative fuels for the most promising options. In the sections below we will examine, for the most promising options, the implications of research improvements in fuel processing.

\subsubsection{Screening of Fuel Option Feasibility}

The allowable algal feedstock costs derived from the fuel product cost goals and the reference processing costs for each of the 45 fuel option cases are presented in Table 6-5 along with the corresponding productivity attainable production cost. The largest gap between productivity attainable production cost and the corresponding allowable feedstock processing cost is at the $5 \%$ photosynthetic efficiency level--the current state of the art in algal production technology. For this low level of algal biomass productivity, even at $50 \%$ lipid content, production costs $(\$ 388 / t)$ far exceed the value of the feedstock for all five fuel process options. It is apparent that current levels of productivity are inadequate for the production of cost-competitive fuels. 


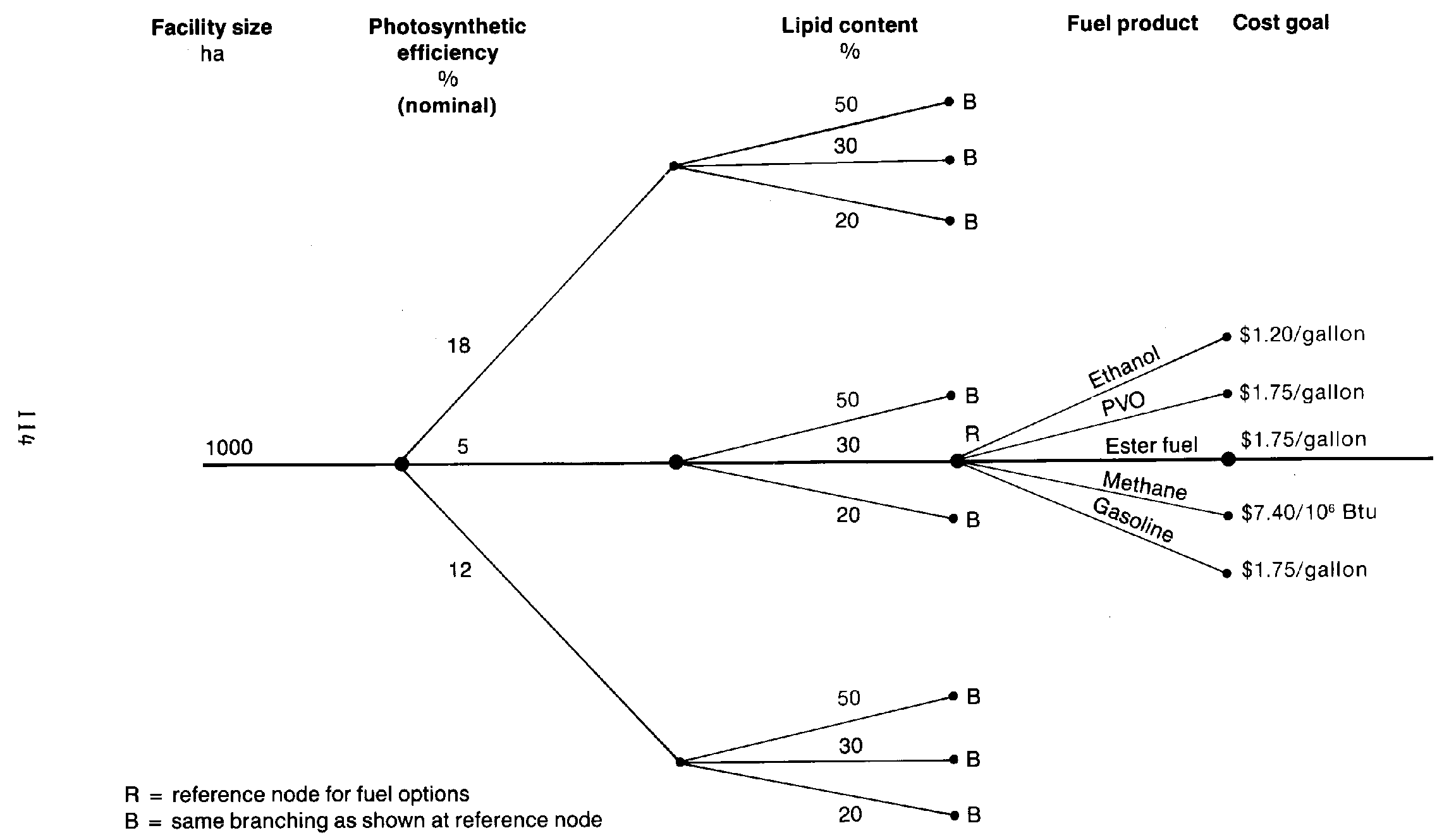

Figure 6-7. Reference, Research Opportunity, and Fuel Option Cases and Parameter Values 
At $12 \%$ photosynthetic efficiency (nominal), the gap between allowable costs and production cost is less, but still too wide to indicate feasibility. At the most optimistic productivity levels--18\% photosynthetic efficiency and $50 \%$ lipid content--production costs exceed fuel values by 5\%-45\%. This comparison involves reference processing cost and potential productivity costs only--we do not incorporate other production cost reductions identified in Section 6.1. Thus, we are testing whether microalgae productivity improvements (the so-called productivity attainable production cost) alone yield costcompetitive fuel production. The result is that productivity improvements alone do not, at least within the constraints imposed by the fuel cost goals, achieve the costcompetitiveness goal.

Some preliminary conclusions about the various fuel options can be made from the data in Table 6-5. First, feedstocks of greater lipid content are always of higher value for fuels that utilize primarily the lipid content (PVO, ester fuel, and gasoline). This is to be expected because the structure of the lipids is conducive to producing higher-valued fuels, and the remaining components are conducive to lesser-value fuels (methane or fuel gas) or to by-products. The greater the fraction of lipids in the feedstock, the higher the value of the feedstock in fuel production. One exception is the production of ethanol, which utilizes only the carbohydrate fraction of the algal feedstock. In the best case examined in terms of carbohydrate content, the lipid content is $20 \%$ and the corresponding carbohydrate content is $49 \%$. Since carbohydrate levels of $70 \%$ have been reported to be attainable, in the next section we will look at ethanol as a special case in which both production conditions and processing design are optimized for carbohydrate and ethanol production.

Comparison of alternative fuel options for various levels of productivity indicates that methane processes consistently have lower feedstock values relative to other fuels. While all of the fuel processes have a methane process attached to convert wastes to fuel, the process that uses the feedstock exclusively for methane does not compare well against the other fuel options. The relative price target of methane, $\$ 7.40 / 10^{6} \mathrm{Btu}$, is about half that of the other fuels; therefore, despite high conversion rates, allowable feedstock costs are lower for methane-only processes. Moreover, examination of the other fuel processing options indicates that as the algal composition switches from carbohydrates and protein (methane-only substrate) to lipids, process economics improve. Thus, it appears that the high cost of producing algal feedstocks rules out production of methane as the primary fuel product. Feasible technology schemes must produce higher valued products from the lipid fraction to offset the high cost of producing algal feedstocks.

Based on the data in Table 6-5, we can eliminate several of the experimental design cases from further evaluation. First, all cases with $20 \%$ lipid content exhibit discrepancies between production cost and feedstock values of a factor of 2 or more and all evidence indicates that higher lipid content is needed to achieve cost effectiveness. Second, PSE levels of 5 and 12 (nominal) appear to be too low to merit extensive evaluation, so we eliminate these cases in order to concentrate on higher levels of productivity. Third, cost-effective alcohol production depends on high carbohydrate content of the algae, so we can limit the evaluation of alcohol fuel feasibility cases to those of high carbohydrate content. And, as noted above, methane from algae appears to be not viable, so we eliminate combinations that involve methane processing as the primary product.

The combined effect of screening the initial 45 options originally proposed leaves 4 cases for further analysis--the two initial lipid content levels ( $30 \%$ and $50 \%$ ), an intermediate lipid level of $40 \%$ to allow for greater specification of the lipid response function, and a 
special case constructed to evaluate ethanol productions. These cases are listed in Table 6-6 where each case is associated with an alphanumeric indicator to indicate the fuel type ( $L=$ lipid-based fuels; $A=$ alcohol) and the corresponding level of the primary chemical content (30,40, and 50 for $L$ cases and A 60 case referring to the algal lipid and carbohydrate content). With this small set of cases, we can concentrate on evaluating the value of improvements in the processing of microalgae and then integrate production and processing improvements to derive a set of technology targets that meet the operative definition of cost effectiveness--achieving fuel production at 2010 cost goal levels.

\subsection{Attainability Targets for Microalgae Feedstock Production and Fuel Processing}

The objective of this section is to match sets of algal production parameters with sets of consistent processing values such that a cost-effective system is defined. Sensitivity evaluations of previous sections suggest the degree to which individual parameter variations improve costs and how clusters of parameters can be varied to represent likely situations. The large number of parameters, most of which are measured on a continuous scale, mean that the solution space is dense--a large number of parametric combinations may meet the criteria of equality of algal production and allowable feedstock cost. Choosing among these for technically feasible sets is a necessary condition for establishing attainability targets to guide technology research and development objectives and priorities.

Solving for attainability configurations is confounded by the subjective nature of costeffective solutions--they invariably include expectations and judgments about future economic conditions and about the attainability of technology advancements. We cannot hope to enumerate all solutions available since they number in the thousands. An alternative is to evoke standard operations research procedures to explore the simulated solution space based on an objective function. This approach is inappropriate in this evaluation due to the indeterministic nature of the models data and functional relationships and because it is premature to specify the required objective function.

Given the objective of this technology evaluation, to define the limits of and needs for technology improvement consistent with achievement of a cost-effective system, we must be content with providing a perspective on needed improvements wide enough to allow for constructing meaningful tradeoffs among options. This section approaches attainability through the use of a first approximation to an "attainable" technology configuration with subsequent variation of the subjective elements of that configuration,

Table 6-6. Productivity and Fuel Type Cases for Algal Processing Evaluation

\begin{tabular}{lccc}
\hline Case & $\begin{array}{c}\text { Carbohydrate } \\
\text { Content }\end{array}$ & $\begin{array}{c}\text { Lipid } \\
\text { Content }\end{array}$ & PSE \\
\hline L30 & 20 & 30 & 14.18 \\
L40 & 16 & 40 & 15.17 \\
L50 & 12 & 50 & 16.18 \\
A60 & 60 & 2 & 10.35 \\
\hline
\end{tabular}


enumeration of their impact, and identification of mitigating instruments--ways of overcoming constraints by improvement of additional aspects of the technology. This approach will not exhaust the full set of possible solutions but will provide a context from which technology analysts and scientists can construct a technology development program.

\subsubsection{An Attainable Production Technology Configuration}

An attainable production technology configuration is defined as a fully specified production scheme, with single values for all parameters, that meets a specific criteria for cost-effectiveness. This attainable technology configuration can then be varied according to important dimensions and by manipulation of various controllable elements. The attainable configuration is defined by specific levels of resource, facility design, and biological and financial parameters: To define an attainable feedstock production concept, we must select levels for all variables that define the system. Below we review the categories of model parameters and indicate our initial specification of attainability.

\section{Resource Parameters}

The large sensitivity of production cost to growing season length, illustrated in Section 5.0, is an obvious area for improvement. Achievement of a season longer than the reference 250 days per year rests on a number of improvements in the system. Greater temperature tolerance of culture algae is needed to endure seasonal temperature fluctuations. In the absence of covers to keep heat in during cool weather and ventilation to prevent elevated temperatures in hot weather, temperature tolerance improvements can be considered an innate characteristic of the algae themselves and one that must be developed as part of the species selection, development, and adaptation program.

An accurate representation of temperature fluctuations and growing season effects requires a more disaggregate representation of time--the annual frame of the existing model can only reflect averages over all seasons and conditions. For the purposes here, an annual evaluation will have to suffice using average sunlight energy $\left(5000 \mathrm{kcal} / \mathrm{m}^{2} / \mathrm{d}\right)$ and a growing season of 300 days, representative of desert Southwest production for 10 months (February through November).

Another attainability target is lower $\mathrm{CO}_{2}$ cost, which speaks more to the site selection and efficient carbon utilization, recycling, and uptake. Extensive development of enhanced oil recovery operations in the southwest could leave the area with an extensive transportation network and developed supplies available at low cost. Alternatively, one could speculate that the pristine, undeveloped nature of prime algal production regions in the southwest persists into the 21 st century and algal facilities must construct their own infrastructure for $\mathrm{CO}_{2}$ and electricity, in which case costs could be higher than the reference case.

Carbon dioxide cost for the attainability case is set at $\$ 3.00$ per thousand standard cubic feet $\left(\$ 0.10 / \mathrm{m}^{3}\right)$, a reduction of about $23 \%$ from the reference case. This reduction could come about as a result of lower acquisition costs or from reduced outgassing and recycle of carbon (Weissman 1985), or both. 


\section{Facility Design Parameters}

Large reductions in algal production cost can only feasibly come about with improvements in the PSE through higher densities and shorter detention times. For the attainability case, we lower the detention time from the reference level of 7 days to 3 and raise the culture density from 800 to $1000 \mathrm{mg} / \mathrm{L}$. Culture densities of $1000 \mathrm{mg} / \mathrm{L}$ can be achieved by good hydraulics that circulate the algae into and out of the light, thereby making fuller use of the available sunlight. Shorter detention times require more vigorous, faster-growing algae, a property that must be developed, and through species selection and development combined with system optimization of the culture environment. This attainability target is under the control of scientists, provided it is technically feasible.

\section{Financial Parameters}

Future economic conditions are difficult to forecast accurately and generally are beyond the control of technology development activities. These variables establish the conditions within which the technology operates. All financial parameters are held constant except the return on debt. The base rate of return on owner capital, $4.5 \%$ (real), is low for highly speculative investments. Although we do not expect a 1000 hectare facility to be proposed, financed, and constructed until the technology feasibility has been established at a pilot scale and the near-term horizon for economic success is favorable, higher returns may be required for the first generation of facilities to of fset inherent risks. In the spirit of constructing a "believable" set of attainability parameters, we raise the return on debt from $4.5 \%$ to $6.5 \%$, which raises financing costs over the reference case that must be offset by physical parameter improvements.

\section{Biological Parameters}

Attainability, as defined by the parameters above, is further partitioned by the assumed cell lipid content into three cases, L30, L40, and L50 corresponding to 30\%, 40\%, and $50 \%$ lipid content with corresponding adjustments in the carbohydrate and nitrogen content. In addition, salinity tolerance is raised to $80 \mathrm{~g}$ TDS/L (from the reference level of 35). Since this characteristic was demonstrated above as having substantial value in overcoming resource constraints, this level of salinity tolerance appears to be a critical target for attainment of an economic system, although relaxing the requirement to $50 \mathrm{~g}$ TDS/L would offer sufficient water related insurance for most situations.

\section{Attainability Costs}

Full detail of the attainability algal production cases, relative to the reference case, is presented in Table 6-7, and resulting production cost and summary data for the cases are in Table 6-8. For all three levels of lipid content except for the ethanol case A60, the cost of production is below $\$ 200 / \mathrm{t}$, ranging from $\$ 192 / \mathrm{t}$ to $\$ 199 / \mathrm{t}$ and lipid costs range from $\$ 93$ to $\$ 54 / \mathrm{bbl}$, the lowest cost associated with $50 \%$ lipids. The allowable cost of these feedstocks must be derived from evaluation of the processing options, the topic of the next section. 
Table 6-7. Parameter Levels for Attainability Microalgae Production Cases: Changes from Reference Case

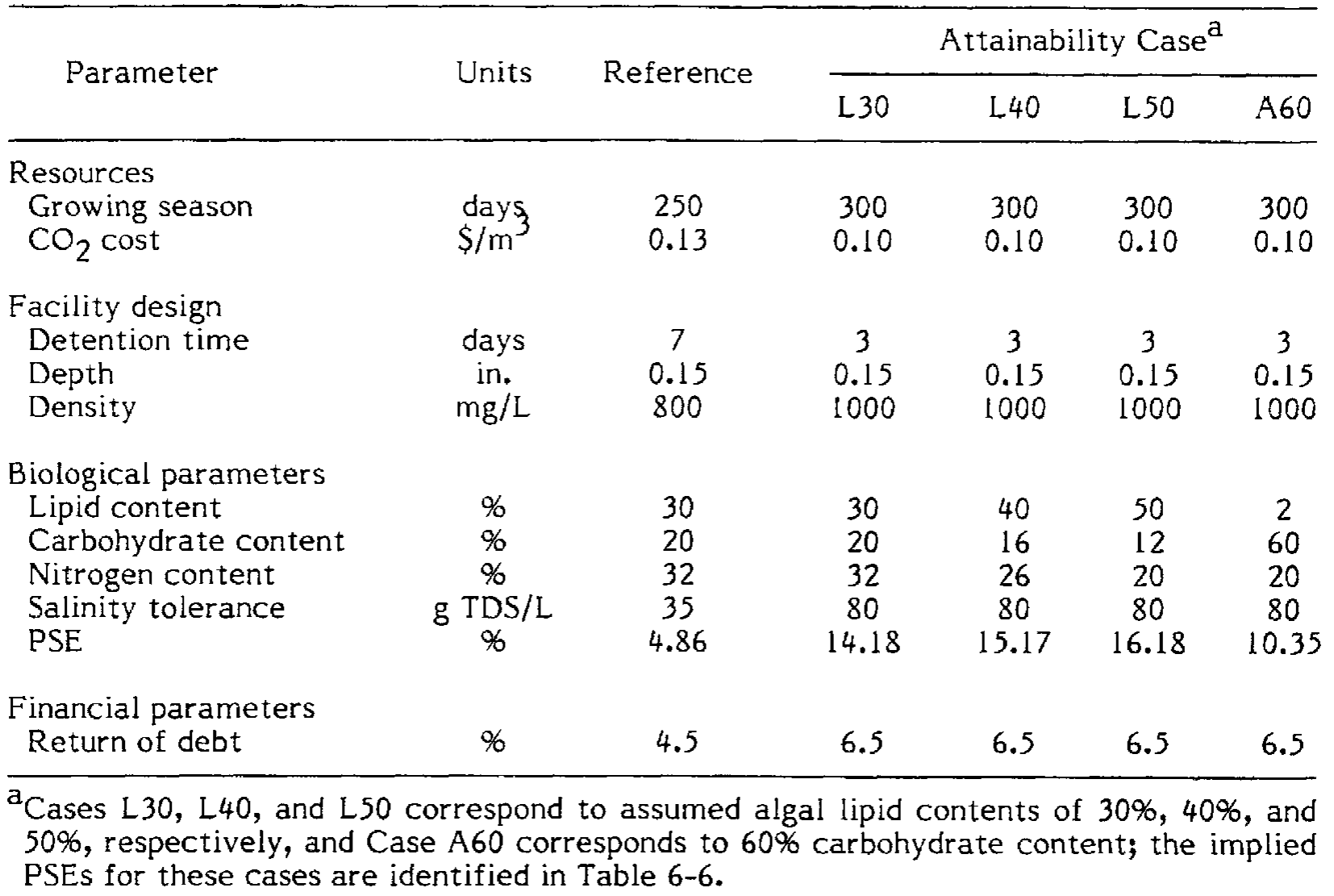

\subsubsection{Fuel Processing}

Processing costs can be reduced, and allowable feedstock cost increased, by increased process conversion efficiency and by the use of larger scale processing facilities. The conversion efficiencies used in the reference cases were derived from preliminary evaluation of the feedstock composition and utilization of available conversion technology. Directed process research and development may be able to significantly raise lipid conversion efficiencies for PVO, ester fuel, and gasoline technologies. These improvements increase the proportion of the feedstock converted to high-value, primary fuels while reducing lower-valued by-product production. For PVO and ester fuel processes, the fuel yield from $50 \%$ lipid feedstocks is raised from $50 \%$ to $75 \%$ and for gasoline from $80 \%$ to $90 \%$. These improvements assume that lipid-rich feedstocks are mostly storage lipids, which are highly conducive to processing.

Scale economies can be expected if larger feedstreams are available. The fuel outputs from the reference cases, based on the feedstock produced on a 1000-ha facility, range from about 1.4 million gallons for PVO, ester fuel, and gasoline to 600,000 gallons for ethanol. By commercial standards, these are very small processing plants that do not take advantage of scale economies that are usually available in chemical and fuel processing. Thus, we raise the scale of the processing plants to approximately ten times that of the reference case, again evoking an assumption in which several colocated algal production facilities are linked to a large processing facility. 
Table 6-8. Summary Output for Attainability Microalgae Production Cases

\begin{tabular}{lccccc}
\hline & & \multicolumn{4}{c}{ Attainability Case } \\
\cline { 4 - 6 } \multicolumn{1}{c}{ Parameter } & Units & L30 & L40 & L50 & A60 \\
\hline System algal yield & $10^{3} \mathrm{t} / \mathrm{yr}$ & 116 & 116 & 116 & 116 \\
Productivity & $\mathrm{g} / \mathrm{m}^{2} / \mathrm{d}$ & 50 & 50 & 50 & 50 \\
Specific growth rate & $\mathrm{d}$ & 0.37 & 0.37 & 0.37 & 0.37 \\
PSE & $\% \mathrm{PAR}$ & 14.18 & 15.17 & 16.18 & 10.35 \\
$\mathrm{CO} 2$ demand & $10^{9} \mathrm{scf} / \mathrm{y}$ & 3.24 & 3.24 & 3.24 & 3.24 \\
Water demand & $10^{6} \mathrm{~m}^{3} / \mathrm{g}$ & 118 & 118 & 118 & 118 \\
Capital investment & $10^{6} 1984 \$$ & 41.08 & 41.08 & 41.08 & 41.08 \\
Operating cost & $\$ / \mathrm{hab}$ & 48 & 48 & 48 & 48 \\
Algal production cost & $10^{6} 1984 \$$ & 17.32 & 16.94 & 16.59 & 16.59 \\
Lipid production cost & $1984 \$ / \mathrm{t}$ & 199 & 195 & 192 & 192 \\
\hline
\end{tabular}

${ }^{\mathrm{a}}$ Cases L30, L40, and L50 correspond to assumed algal lipid contents of $30 \%, 40 \%$, and $50 \%$, respectively.

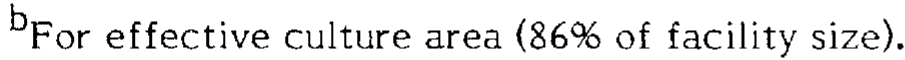

Reference case and attainability values for microaigae processing cases L 30, L40, and L50 and A60 are listed in Table 6-9. The improved ethanol efficiency comes from the assumption that, in addition to the carbohydrate fraction which is hydrolyzed to glucose, the remaining carbohydrates are hydrolyzed to xylose, which also may be convertible to ethanol. Recall also that we constructed a special case, not used in the reference evaluation, where $60 \%$ of the algal biomass is carbohydrate at the highest rate of photosynthetic efficiency. This best attainable ethanol case is constructed to determine the maximum potential for production of ethanol from algal biomass.

Mass and energy balances for the attainability fuel processing concepts are presented in Table 6-10, and fuel and by-product outputs are listed in Table 6-11. By increasing processing efficiencies and raising the scale of the processing plants (to serve a total of 8,000-10,000 ha of microalgae production), major product yields more closely represent commercial design criteria for production level--annual output exceeds 80 mitlion gallons of primary fuel for PVO, ester fuel, and gasoline.

Estimated plant capital costs are illustrated in Figure 6-8 and operating costs in Figure 6-9. Total fuel processing capital costs (Table 6-12) range from about $\$ 70$ million for PVO to over $\$ 160$ million for ester fuel processing plants. The complexity of the transesterification (ester fuel) process, which requires multistage operation and interstep solvent recovery and catalyst neutralization, results in estimated capital costs higher than the other two processes. We caution the reader that fuel process configurations, and therefore costs, are based on preliminary data of feedstock composition; considerable evaluation of process kinetics is needed before more detailed data can be developed. 
Table 6-9. Reference Case and Attainability Values for Lipid Processing Plant Scale and Conversion Efficiency

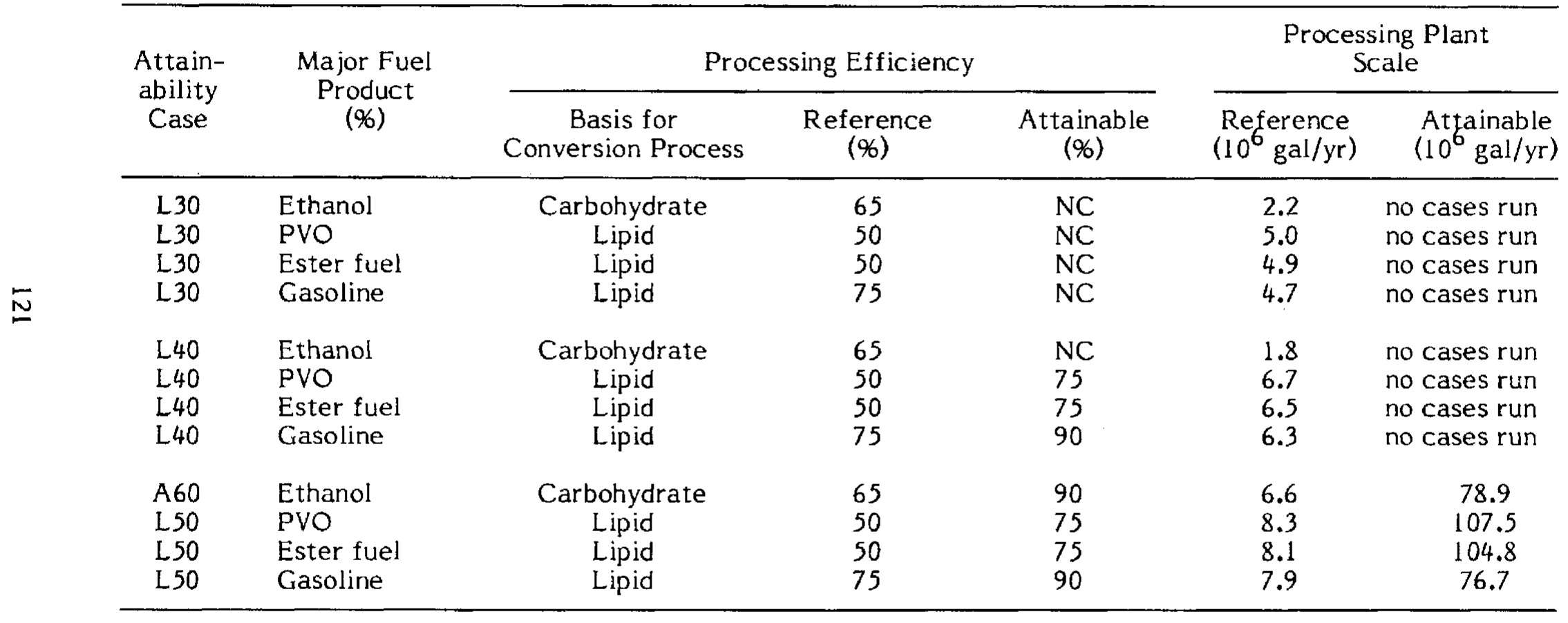

Notes: Efficiency and processing scale attainability values are not varied for different levels of photosynthetic efficiency since the major variation in scale refers to lipid content, and efficiencies are independent of product stream size. 
Table 6-10. Summary of Attainability Product Yields

\begin{tabular}{|c|c|c|c|c|}
\hline & PVO & $\begin{array}{l}\text { Ester } \\
\text { Fuel }\end{array}$ & Gasoline & Ethanol \\
\hline Major algal component & Lipid & Lipid & Lipid & Carbohydrate \\
\hline Fraction of total algae & 0.50 & 0.50 & 0.50 & 0.60 \\
\hline $\begin{array}{l}\text { Fraction available for } \\
\text { conversion }\end{array}$ & 0.75 & 0.75 & 090 & 0.90 \\
\hline Efficiency of utilization & 1.00 & 0.98 & $0.90^{\mathrm{a}}$ & $0.86^{b}$ \\
\hline Production $(t / t$ algae $)$ & 0.38 & 0.35 & 0.21 & 0.24 \\
\hline Common unit/t algae & $107.5 \mathrm{gal}$ & $104.8 \mathrm{gal}$ & $76.7 \mathrm{gal}$ & $78.9 \mathrm{gal}$ \\
\hline $\begin{array}{l}\text { Product energy content } \\
(\mathrm{GJ} / \mathrm{t})\end{array}$ & 39.50 & 36.90 & 43.86 & 26.70 \\
\hline $\begin{array}{l}\text { Main product energy content } \\
(\mathrm{GJ} / \mathrm{t} \text { algae })\end{array}$ & 14.8 & 12.9 & 9.18 & 6.40 \\
\hline $\begin{array}{l}\text { Total energy available } \\
\text { (GJ/t algae) }\end{array}$ & 28.44 & 28.44 & 28.44 & 18.19 \\
\hline $\begin{array}{l}\text { Recovery efficiency } \\
\text { (major product) }\end{array}$ & $52.0 \%$ & $45.4 \%$ & $32.3 \%$ & $35.2 \%$ \\
\hline
\end{tabular}

Table 6-11. Production Summary for Attainability Microalgae Refinery Options

\begin{tabular}{|c|c|c|c|c|}
\hline \multirow{2}{*}{ Process Product (unit) } & \multicolumn{4}{|c|}{ Major Products } \\
\hline & PVO & Ester Fuel & Gasoline & Ethanol \\
\hline Production $\left(10^{6} \mathrm{gal} / \mathrm{yr}\right)$ & 107.5 & 104.8 & 76.7 & 78.9 \\
\hline $\begin{array}{l}\text { Other Products } \\
\text { Methane }\left(10^{15} \mathrm{~J} / \mathrm{yr}\right)\end{array}$ & 4.7 & 4.7 & $4.1^{b}$ & 2.0 \\
\hline $\mathrm{CO}_{2}\left(10^{6} \mathrm{~m}^{3} / \mathrm{yr}\right)$ & 85 & 85 & 50 & 130 \\
\hline $\begin{array}{l}\text { Nitrogen }\left(10^{3} \mathrm{t} / \mathrm{yr}\right) \\
\text { as } \mathrm{NH}_{3}\end{array}$ & 16.9 & 16.9 & 14.9 & 12.5 \\
\hline Glycerol $\left(10^{3} \mathrm{t} / \mathrm{yr}\right)$ & -- & 40 & -- & - \\
\hline $\begin{array}{l}\operatorname{LPG}\left(10^{15} \mathrm{~J} / \mathrm{yr}\right) \\
\left(10^{6} \mathrm{gal} / \mathrm{yr}^{\mathrm{a}}\right)\end{array}$ & $\begin{array}{l}-- \\
--\end{array}$ & $\begin{array}{l}-- \\
--\end{array}$ & $34^{3.7}$ & -- \\
\hline $\begin{aligned} \text { Diesel } & \left(10^{15} \mathrm{~J} / \mathrm{yr}\right) \\
& \left(10^{6} \mathrm{gal} / \mathrm{yr}\right)\end{aligned}$ & -- & -- & 1.8 & -- \\
\hline
\end{tabular}




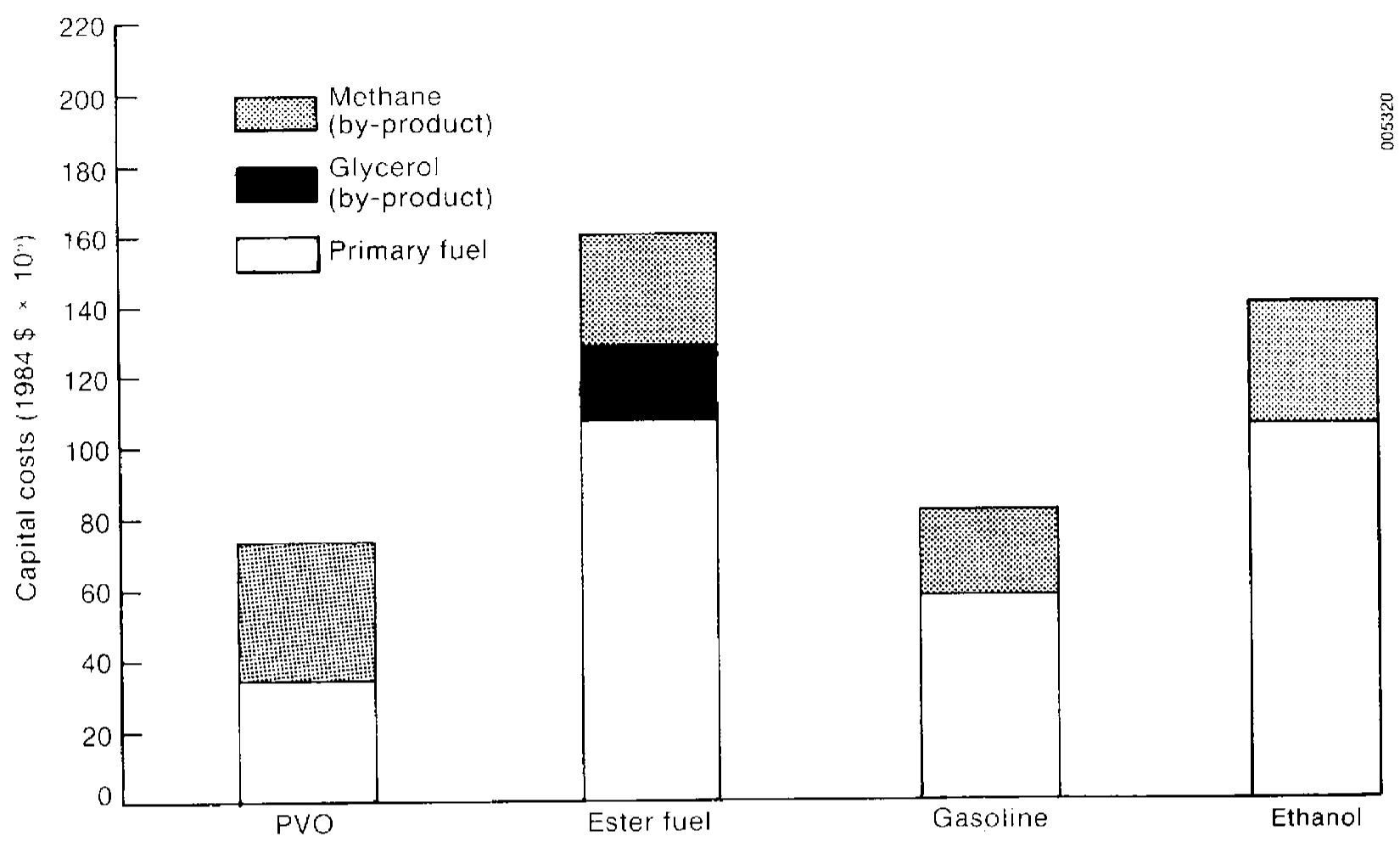

Figure 6-8. Capital Costs for Attainability Fuel Processing Plants

Operating costs for the attainability fuel processes, illustrated in Figures 6-9 and 6-10 are representative of commercial chemical and fuel processes. By achieving all the highest possible conversion rates, feedstock cost becomes the largest single fraction of total operating cost. The success of the fuels-from-microalgae concept depends on the development of processes that can meet the standards imposed by the fuel processing industry, including capital-intensive, large-scale plant designs operating at high efficiency.

\subsubsection{Analysis of Feedstock Production and Fuel Processing Requirements}

Now that we have screened algal production options to the most promising cases and have quantified the impacts of improvements in the processing of algal feedstock for fuels, the two stages of production can be integrated to derive conditions consistent with our measure of cost effectiveness. As before, we fix the value of all fuel products and processing coproducts at 2010 levels and compare the value of the feedstocks implied by a particular processing concept with the cost of producing the feedstock, seeking equality of these values.

Table 6-13 presents the integrated attainability algal production costs (the cases L 30 , L40, L50, and A60 with improvements in other parameters as defined in Table 6-8) and scale, process, and combined improvements in processing described in Table 6-9. 

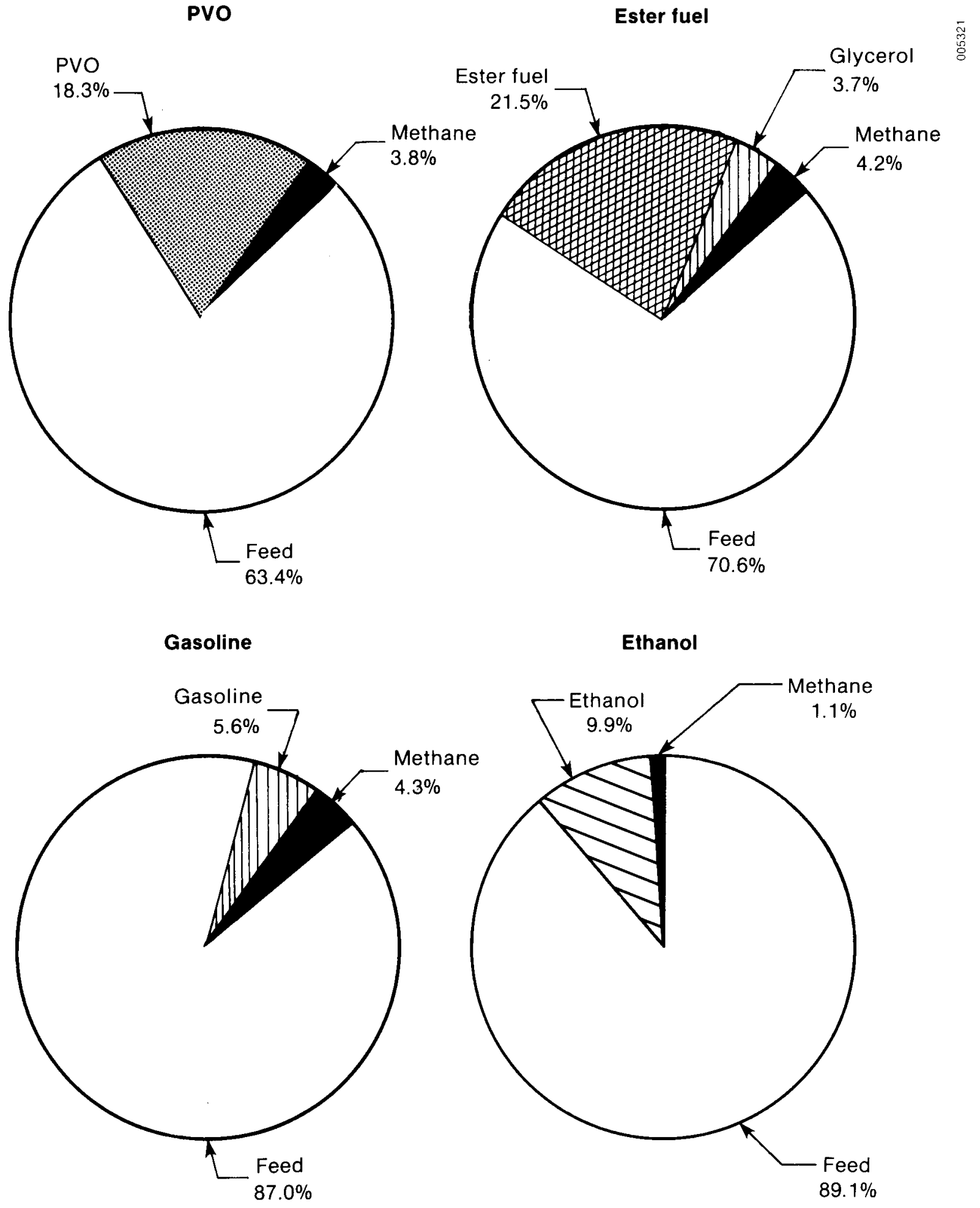

Figure 6-9. Total Operating Costs for Attainability Fuel Processing Plants 
Table 6-12. Summary of Attainability Capital and Operating Costs for Fuel Processing Options

\begin{tabular}{|c|c|c|c|c|}
\hline \multirow{2}{*}{ Cost Category } & \multicolumn{4}{|c|}{ Process } \\
\hline & PVO & Ester Fuel & Gasoline & Ethanol \\
\hline \multicolumn{5}{|l|}{ Capital costs $\left(\$ 10^{6}\right)$} \\
\hline Main process unit & 36.7 & 109.0 & 58.2 & 113.0 \\
\hline Glycerol by-product unit & -- & 17.7 & -- & -- \\
\hline Methane by-product unit & 33.0 & 33.0 & 27.9 & 26.3 \\
\hline Total & 69.7 & 159.7 & 86.1 & 139.3 \\
\hline \multicolumn{5}{|l|}{ Operating costs $\left(\$ 10^{6} / \mathrm{yr}\right)$} \\
\hline Raw materials & 192.3 & 210.2 & 192.1 & 193.1 \\
\hline Electric power & 3.4 & 3.7 & 4.0 & 2.1 \\
\hline Water & 0.2 & 1.3 & 0.2 & 2.3 \\
\hline Steam & 4.1 & 3.5 & 4.1 & 14.2 \\
\hline Labor, maintenance, taxes & 92.1 & 31.9 & 7.8 & 16.5 \\
\hline Depreciation & 7.4 & 16.4 & 8.9 & 13.9 \\
\hline Return on investment & 3.4 & 5.1 & 3.4 & 4.0 \\
\hline Total (gross) & 302.9 & 272.1 & 220.5 & 246.1 \\
\hline \multicolumn{5}{|c|}{ Credits from product sales $c\left(\$ 10^{6} / \mathrm{yr}\right)$} \\
\hline Carbon dioxide & $(7.2)$ & $(7.2)$ & $(4.8)$ & $(10.8)$ \\
\hline Water & $(0.3)$ & $(0.3)$ & $(0.3)$ & $(0.2)$ \\
\hline Nitrogen & $(3.1)$ & $(3.1)$ & $(2.7)$ & $(2.3)$ \\
\hline Methane & $(33.1)$ & $(33.1)$ & $(28.8)$ & $(16.5)$ \\
\hline LPG & 0.0 & 0.0 & $(32.4)$ & - \\
\hline Diesel & 0.0 & 0.0 & (22.1) & - \\
\hline Glycerol & 0.0 & $\underline{(59.2)}$ & 0.0 & - \\
\hline Subtotal (credits) & $(43.7)$ & $(99.8)$ & $(91.1)$ & $(29.6)$ \\
\hline Net operating cgst & 259.2 & 172.3 & 129.4 & 216.3 \\
\hline Fuel production & 107.5 & 104.8 & 76.7 & 78.9 \\
\hline Main product cost $(\$ / g a l)$ & 2.40 & 1.65 & 1.70 & 2.75 \\
\hline
\end{tabular}

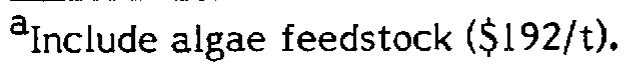

$\mathrm{b}_{\text {Process and cooling water. }}$

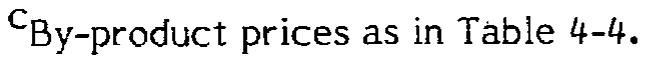

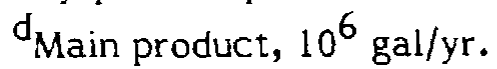

Appropriate comparisons, seeking equality of value, are between columns 4, 5, 6 (scale, process, and both processing improvements) and column 7, the production cost associated with the feedstock assumed for each processing configuration and primary fuel type (Table 6-13).

Our evaluation of the data in Table 6-13 will be by fuel type, beginning with ethanol (case A60). None of the individual processing improvements (columns 3 and 4) achieves 

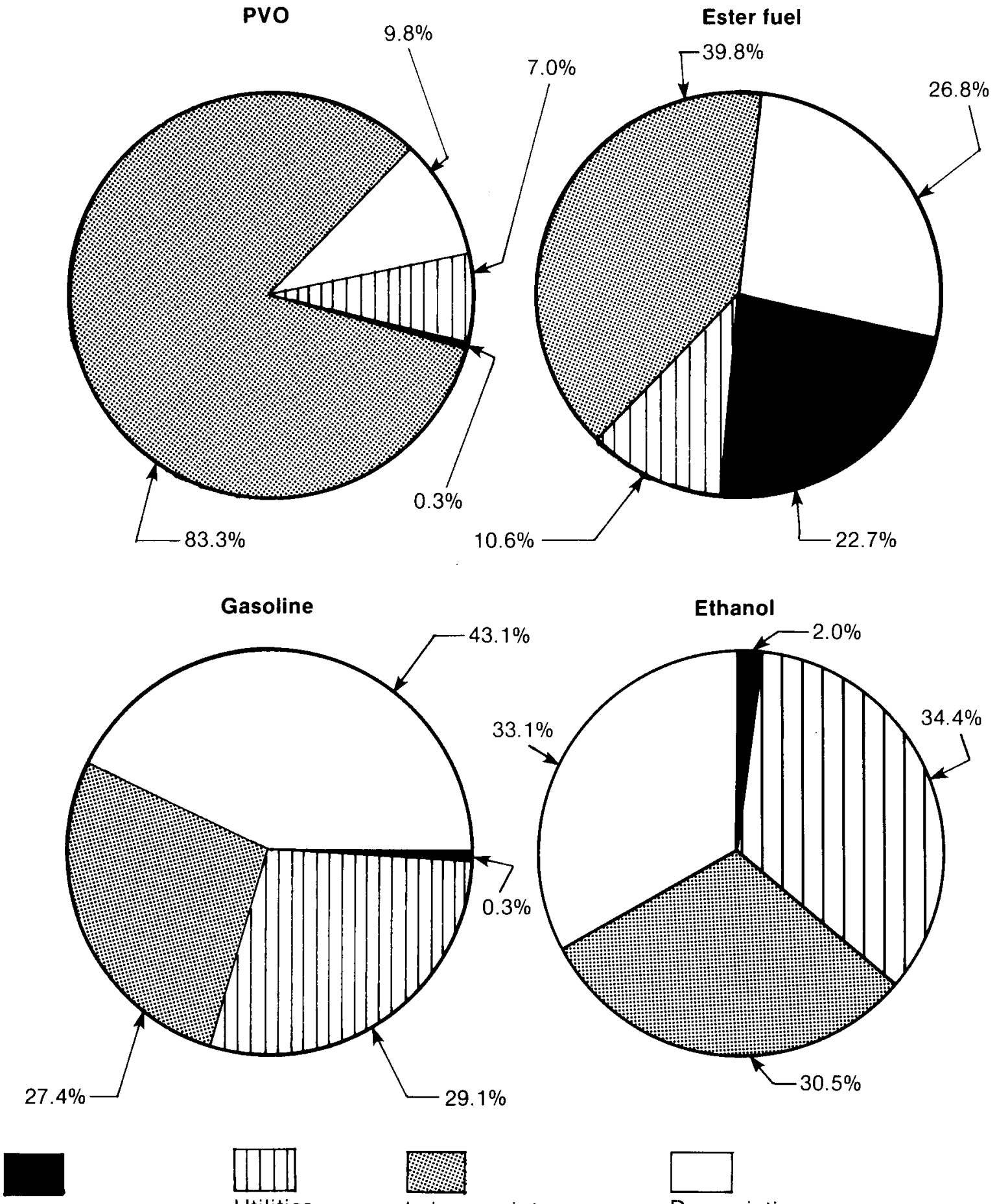

Raw materials
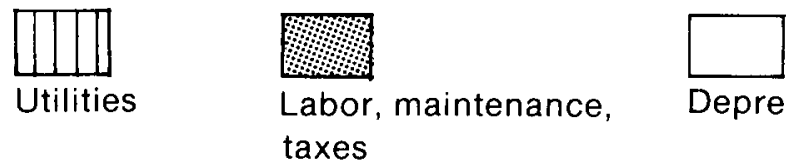

Depreciation taxes

Figure 6-10. Operating Costs (Excluding Feedstock) for Attainability Fuel Processing Plants 
Table 6-13. Comparison of Integrated Allowable Costs and Production Costs for Microalgae-Derived Fuels

\begin{tabular}{|c|c|c|c|c|c|c|}
\hline \multicolumn{2}{|c|}{ Fuel and Production Case } & \multicolumn{5}{|c|}{ Allowable Algal Feedstock Processing Cost $(\$ / t)$} \\
\hline $\begin{array}{l}\text { Primary } \\
\text { Fuel Case } \\
\text { (1) }\end{array}$ & $\begin{array}{l}\text { Attainable } \\
\text { Production } \\
\text { Case } \\
\text { (2) }\end{array}$ & Reference $^{b}$ & $\begin{array}{c}\text { Scale } \\
\text { Onlyc } \\
(4)\end{array}$ & $\begin{array}{l}\text { Attainable } \\
\text { Process Improye- } \\
\text { ments Only } \\
\text { (5) }\end{array}$ & $\begin{array}{c}\text { Scale and } \\
\text { Attainable Process } \\
\text { Improvements } \\
(6)\end{array}$ & $\begin{array}{c}\text { Integrated } \\
\text { Attainability } \\
\text { Production Cost } \\
(7)\end{array}$ \\
\hline Ethanol & A 60 & 36 & 63 & 39 & 71 & 192 \\
\hline $\begin{array}{l}\text { PVO } \\
\text { PVO } \\
\text { PVO }\end{array}$ & $\begin{array}{l}\mathrm{L} 30 \\
\mathrm{~L} 40 \\
\mathrm{~L} 50\end{array}$ & $\begin{array}{l}107 \\
120 \\
132\end{array}$ & $\begin{array}{l}118 \\
131 \\
151\end{array}$ & $\begin{array}{l}115 \\
132 \\
145\end{array}$ & $\begin{array}{l}127 \\
145 \\
165\end{array}$ & $\begin{array}{l}199 \\
195 \\
192\end{array}$ \\
\hline $\begin{array}{l}\text { Ester } \\
\text { Ester } \\
\text { Ester }\end{array}$ & $\begin{array}{l}\text { L } 30 \\
\text { L } 40 \\
\text { L } 50\end{array}$ & $\begin{array}{l}108 \\
124 \\
141\end{array}$ & $\begin{array}{l}131 \\
151 \\
171\end{array}$ & $\begin{array}{l}122 \\
145 \\
170\end{array}$ & $\begin{array}{l}150 \\
177 \\
206\end{array}$ & $\begin{array}{l}199 \\
195 \\
192\end{array}$ \\
\hline $\begin{array}{l}\text { Gasoline } \\
\text { Gasoline } \\
\text { Gasoline }\end{array}$ & $\begin{array}{l}\text { L } 30 \\
\text { L } 40 \\
\text { L } 50\end{array}$ & $\begin{array}{l}123 \\
144 \\
166\end{array}$ & $\begin{array}{l}138 \\
161 \\
184\end{array}$ & $\begin{array}{l}128 \\
152 \\
177\end{array}$ & $\begin{array}{l}143 \\
169 \\
197\end{array}$ & $\begin{array}{l}199 \\
195 \\
192\end{array}$ \\
\hline
\end{tabular}

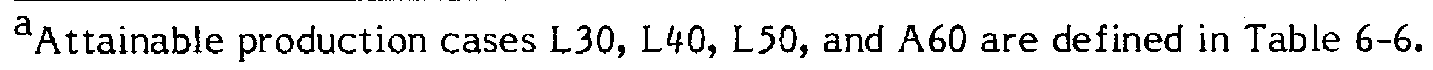

${ }^{b}$ Reference processing throughput is $116,000 \mathrm{t} / \mathrm{yr}$.

${ }^{\mathrm{C}}$ Scale processing throughput is $1,000,000 \mathrm{t} / \mathrm{yr}$ ( 9 plants).

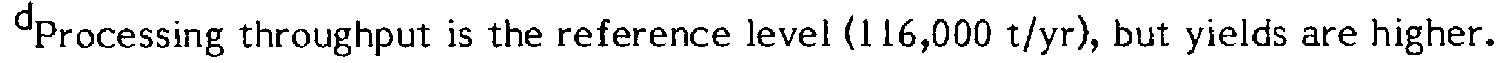

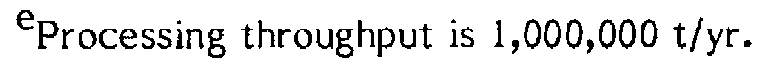


equality of algal feedstock production cost and processing value, and even assuming all possible processing improvements (column 6), the production cost exceeds the processing value (column 7) by a factor of almost three. The high cost of producing carbohydrates by algal culture appears to preclude the cost-effective production of alcohol, given the 2010 alcohol cost targets established for this analysis. This conclusion can be seen from another perspective by comparing the ethanol cost goal for 2010 with the ethanol cost implied by processing algal feedstock costing $\$ 71$, the best case evaluated. Table 6-14 shows that ethanol prices would have to exceed the 2010 cost goal by about $\$ 1.55$ for this technology to be cost effective. Since current expectations for fuel prices equivalent to $\$ 2.75$ ethanol are beyond 2020 , it appears that ethanol from microalgae is a technology with, at best, a distant opportunity and therefore deserving a low priority for research and development.

The prospects for PVO are not as dismal as those for ethanol, but this fuel option also fails our cost-effectiveness test. Even with the most favorable realization of production (L50) and processing improvements (both scale and processing improvements), the production cost exceeds the processing value by $14 \%$, implying that technology feasibility would occur sometime after 2010 and, as we see below, this process is inferior to other options and therefore may never become attractive as a commercial technology. Again, the value of research directed at this option appears low.

Ester fuel suggests the possibility of becoming cost effective but only if all possible improvements are realized. As Table 6-14 indicates, realization of these conditions results in production costs of $\$ 0.10$ below the cost goal level and thus our cost-effective criterium is exceeded. The same is true for gasoline from microalgae, again, in the case where all specified production and processing improvements are achieved.

The result of our extensive screening and integrated production and process analysis suggests that two fuels show promise of cost effectiveness by the first decade of the 21st century. However, ester fuel and gasoline can achieve this distinction only if the full set of technology improvements specified above are achieved. Specifically, algal production must achieve a PSE of slightly above $16 \%$ with $50 \%$ lipid content and production must find conditions that support extenşive carbon dioxide and water resources with costs at or below $\$ 0.10 / \mathrm{m}^{3}$ and $\$ 0.13 / \mathrm{m}^{3}$, respectively, and the other reference parameter assumptions must be met. On the processing side, substantial process efficiency

Table 6-14. Derived Microalgae Fuel Production Costs for Integrated Attainability Concept

\begin{tabular}{lcc}
\hline $\begin{array}{l}\text { Fuel } \\
\text { Product }\end{array}$ & $\begin{array}{c}\text { Cost } \\
\text { Goals } \\
\text { (\$/gal) }\end{array}$ & $\begin{array}{c}\text { Attainable } \\
\text { Fuel Cost } \\
\text { (\$/gal) }\end{array}$ \\
\hline PVO & 1.75 & 2.40 \\
Ester fuel & 1.75 & 1.65 \\
Gasoline & 1.75 & 1.70 \\
Ethanol & 1.20 & 2.75 \\
\hline
\end{tabular}


improvements must be achieved and industry scale economies realized to achieve the needed cost level, $\$ 192 / \mathrm{t}$. Tables 6-15 through 6-17 list the important variations from reference assumptions and represent the technology development targets derived from the integrated technology evaluation.

The scale requirement for cost effectiveness appears to describe a chicken-egg dilemma; scale economies are needed for commercial viability but unless first applications are at the required scale (10,000 hectares), they will not be cost effective. This apparent paradox should not be judged as reason to rule out development of this technology since our cost-effectiveness criterium requires that the first year cost meet the exact cost goal for 2010, thus ignoring that later year prices exceed the cost goal and thus losses in the early years could be compensated by later year revenues above costs. Taking the price forecast for 2010 for gasoline, for example, gives a first year price of $\$ 1.75 / \mathrm{g}$, but the process improvement only case implies a production cost of about $\$ 1.90 / \mathrm{g}$, with an implied loss of $\$ 0.15 / \mathrm{g}$. However, as gasoline prices rise from 2010 , this loss is eroded and eventually positive margins accrue to first offset the loss and later contribute to return to investment.

We adopted a stringent first year cost criteria to focus attention on $R \& D$ requirements. However, given the near equality, for gasoline and ester fuel, of production cost and value, it would be remiss not to take into account outyear forecasts in determining technology feasibility. We leave subsequent studies of market penetration rates and the economics of early adoption to another forum.

Table 6-15. Parameter Values for Attainability Microalgae Production: Variances from Reference Case Values

\begin{tabular}{|c|c|c|}
\hline Parameter $^{\mathrm{a}}$ & Reference Value & Attainability \\
\hline $\begin{array}{l}\text { Carbon dioxide cost }\left(\$ / \mathrm{m}^{3}\right) \\
\text { Nitrogen cost }(\$ / \mathrm{t}) \\
\text { Phosphorus cost }(\$ / \mathrm{t}) \\
\text { Potassium cost }(\$ / \mathrm{t}) \\
\text { Electricity cost }(\$ / \mathrm{kWh})\end{array}$ & $\begin{array}{l}0.13 \\
203 \\
282 \\
102 \\
0.05\end{array}$ & $\begin{array}{l}0.10 \\
183 \\
253 \\
92 \\
0.03\end{array}$ \\
\hline $\begin{array}{l}\text { Productivity }\left(\mathrm{g} / \mathrm{m}^{2} / \mathrm{d}\right) \\
\text { Algal composition } \\
\text { Lipids (\%) } \\
\text { Carbohydrate (\%) } \\
\text { Protein }(\%) \\
\text { Potassium (g/g DW) }\end{array}$ & $\begin{array}{l}25 \\
30 \\
20 \\
32 \\
0.007\end{array}$ & $\begin{array}{c}53 \\
50 \\
9 \\
13 \\
0.0012\end{array}$ \\
\hline $\begin{array}{l}\text { Nutrient outgasssing } \\
\text { Carbon }\left(\mathrm{g} / \mathrm{m}^{2} / \mathrm{d}\right) \\
\text { Nitrogen }\left(\mathrm{g} / \mathrm{m}^{2} / \mathrm{d}\right)\end{array}$ & $\begin{array}{l}0.05 \\
0.05\end{array}$ & $\begin{array}{l}0.1 \\
0.1\end{array}$ \\
\hline $\begin{array}{l}\text { Source water composition (g TDS/L) } \\
\text { Algal salinity tolerance }(\mathrm{g} \text { TDS/L) } \\
\text { Carbon dioxide transportation }(\mathrm{km})\end{array}$ & $\begin{array}{l}8 \\
35 \\
80\end{array}$ & $\begin{array}{c}25 \\
80 \\
0\end{array}$ \\
\hline
\end{tabular}


Table 6-16. Input and Output Material Flows for the Integrated Attainability Microalgae Production Concept (1000-ha facility)

\begin{tabular}{|c|c|}
\hline Material Flows & Quantity \\
\hline \multicolumn{2}{|l|}{ Inputs to Microalgae Production } \\
\hline \multicolumn{2}{|l|}{ Resources } \\
\hline $\begin{array}{l}\text { Carbon dioxide }\left(10^{-} \mathrm{m} / \mathrm{yr}\right) \\
\text { Water }\left(10^{6} \mathrm{~m}^{3} / \mathrm{vr}\right)\end{array}$ & $\begin{array}{c}109 \\
12\end{array}$ \\
\hline Nitrogen $(t / y r)$ & 481 \\
\hline Phosphorus (t/yr) & 319 \\
\hline Potassium (t/yr) & 392 \\
\hline Labor (t/yr) & \\
\hline \multicolumn{2}{|l|}{ Energy } \\
\hline Electricity $\left(\mathrm{L}^{6} \mathrm{kWh} / \mathrm{yr}\right)$ & 15 \\
\hline Sunlight ${ }^{\mathrm{a}}\left(10^{9} \mathrm{kcal}\right)$ & 99 \\
\hline \multicolumn{2}{|l|}{ Outputs } \\
\hline Microalgae (t/yr) & 130 \\
\hline Lipids (t/yr) & 78 \\
\hline Water disposal $\left(10^{6} \mathrm{~m}^{3} / \mathrm{yr}\right)$ & 2.6 \\
\hline Brine disposal $\left(10^{-} \mathrm{t} / \mathrm{yr}\right)$ & 210 \\
\hline
\end{tabular}

\subsection{Sensitivity Analysis}

The attainability cases we constructed above are representative of the many combinations and permutations of the model and technology parameters that meet the criteria of an algal production price of $\$ 192 / t$. In this section, we examine variations of the attainability case to see how our concept of attainability withstands alternative assumptions about the assumed economic and resource conditions.

\subsubsection{Sensitivity of the Attainability Production Cost to Constraints}

Cases in this section represent variations in parameters from their level in the first attainability case specified in the last section. The objective of this exercise is to lend a perspective on the stability of "attainability" under alternative assumptions about the state of the economic environment--factors largely beyond the control of the technology development program. These constraints, increases in implicit cost of production, are evaluated in terms of increases in controllable parameters, mainly productivity, needed to mitigate these effects.

Sensitivity cases for increased cost are described in Table 6-18 and cover a variety of factors subject to considerable uncertainty. The levels of parameter changes are intended to give a perspective on the sensitivity of attainability over a range of values that correspond to progressively more stringent constraints, but the particular values are arbitrary--not referenced to a particular site or set of circumstances. 
Table 6-17. Summary of Integrated Attainability Production Facility Cost Contributions (1984 \$)

\begin{tabular}{|c|c|c|c|}
\hline Cost Category & $\$ / t$ & $\begin{array}{l}\text { Percentage } \\
\text { of Category }\end{array}$ & $\begin{array}{l}\text { Percentage } \\
\text { of Total Cost }\end{array}$ \\
\hline \multicolumn{4}{|l|}{ Capital Cost } \\
\hline Culture system & 25.2 & 51.3 & \\
\hline Harvester system & 12.1 & 24.7 & \\
\hline Engineering fees & 4.0 & 8.2 & \\
\hline Contingency & 6.2 & 12.6 & \\
\hline Land & 1.5 & 3.1 & \\
\hline Total capital cost & 49.0 & 100.0 & 25.5 \\
\hline \multicolumn{4}{|l|}{ Operating Costs } \\
\hline Labor and overhead & 20.9 & 13.7 & \\
\hline Utilities & 13.4 & 2.7 & \\
\hline Nutrients & 92.7 & 63.2 & \\
\hline Water & 6.8 & 4.9 & \\
\hline Other operating & 9.8 & 6.5 & \\
\hline Total operating cost & 143 & 100.0 & 74.5 \\
\hline Total feedstock cost & 224.0 & & 100.0 \\
\hline
\end{tabular}

The main factor, under the control of the development program, for mitigating these impacts is algal productivity (PSE). For each case where an individual economic parameter is varied, we sequentially raise the PSE until the production cost is decreased back to the attainability level of $\$ 192 / \mathrm{t}$. Changes in the PSE are induced via increases in the density from the attainability target of 1000 , up to $1400 \mathrm{mg} / \mathrm{L}$, where the PSE exceeds $23 \%$. Further cost decreases, where needed, are achieved by increasing the level of other biological targets (nutrient outgassing, growing season, and the evaporation rate) judged to be more difficult to achieve but which do offer opportunity for improvement, if required.

Table 6-19 and Figure 6-11 present the results of solving for the mitigating level of PSE for each sensitivity case. As defined in Table 6-18, there are 10 primary sensitivity cases (A.1-A.6, B.1-B.2, C.1-C.2), each with two or three subdivisions of the levels of increase or decrease (growing season days are decreased to represent more stringent environmental conditions) for a total of 28 cases. Taking all first level increases as approximately equal increments of increases in the constraint and the same for second and third level increases allows for a subjective evaluation of the need to raise the PSE target above the $16 \%$ attainability level.*

Of the 10 first level increases (the first level change for all cases, column 4 in Table 6-19), two require a PSE increase to less than $16.49 \%$, two more of these increases

* This is a subjective assessment of relative probability across diverse events and is not derived from any formal probabilistic analysis. 
Table 6-18. Sensitivity Cases for Attainable Microalgae Production Cost ${ }^{\mathrm{a}}$ : Uncertainties that Increase Costs

\begin{tabular}{|c|c|c|c|c|c|c|c|}
\hline \multirow[b]{2}{*}{ Case } & \multirow[b]{2}{*}{$\begin{array}{c}\text { Parameter } \\
\text { Type }\end{array}$} & \multirow[b]{2}{*}{ Parameter } & \multirow[b]{2}{*}{ Lnits } & \multirow[b]{2}{*}{$\begin{array}{c}\text { Attaina- } \\
\text { bility }\end{array}$} & \multicolumn{3}{|c|}{ Levels of Change $^{b}$} \\
\hline & & & & & Ist & 2nd & $3 r d$ \\
\hline A.l & Resource & Evaporation rate & $\mathrm{m} / \mathrm{d}$ & 0.0035 & 0.007 & 0.01 & 0.02 \\
\hline A. 2 & Resource & Land cost & $\$ /$ ha & 1245 & 2500 & 5000 & 10000 \\
\hline A.3 & Resource & Water cost & $\$ / m^{3}$ & 0.067 & 0.13 & 0.17 & 0.20 \\
\hline A. 4 & Resource & Carbon dioxide cost & $\$ / m^{3}$ & 0.10 & 0.12 & 0.14 & -- \\
\hline A.5 & Resource & Source water salinity & $\mathrm{g} \mathrm{TDS} / \mathrm{L}$ & 25 & 35 & 50 & -- \\
\hline A.6 & Resource & Growing season & $d / y r$ & 300 & 285 & 270 & 265 \\
\hline B. 1 & Facility design & BOS $\cos t^{c}$ & $\%$ & 0 & 10 & 15 & 20 \\
\hline B.2 & Facility design & Liner cost & \$/ha & 5000 & 7500 & 10000 & 12500 \\
\hline C.1 & Financial & Maintenance cost & $\% \mathrm{CI}^{\mathrm{d}}$ & 7 & 10 & 15 & 20 \\
\hline C. 2 & Financial & Equity capital & $\% / \mathrm{ROI}^{\mathrm{e}}$ & $30 / 6.5$ & $100 / 7$ & $100 / 8$ & $100 / 9$ \\
\hline
\end{tabular}

${ }^{\mathrm{a} C a s e s}$ represent imposition of additional constraints, through higher levels of indicated variables, on the attainability microalgae production concept.

${ }^{b}$ Levels correspond to a second level of indexing of cases, e.g.; A.1.l (1st level), A.l.2 (2nd level), A.1.3 (3rd level).

${ }^{C} \mathrm{~A}$ balance-of-system contingency defined as a percentage of capital cost.

${ }^{\mathrm{d}} \mathrm{Cl}=$ capital investment.

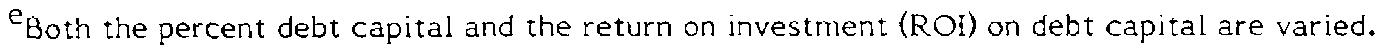

are mitigated by a PSE less than $17 \%$, and all but one (A.4.1, a $16 \%$ increase in $\mathrm{CO}_{2}$ cost) require a PSE of less than $17.5 \%$. Except for the three most constraining cases (A.4.3, D.1.3, and D.2.3), all second and third level increases are mitigated at PSEs below 18.5\%. First we offer some remarks about these cases and then look more closely at the effect of increased $\mathrm{CO}_{2}$ costs.

The most restrictive cases are those requiring increases above $18.50 \%$ PSE. Extreme cases that are still below that threshold include evaporation rates up to $0.02 \mathrm{~m} / \mathrm{d}$ (twice

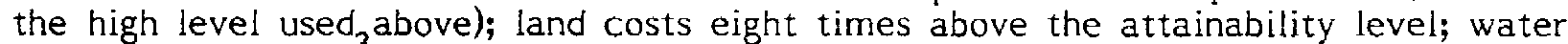
costs up to $\$ 0.20 / \mathrm{m}^{3}$ (also the high level); a growing season as low as 265 days; liner costs two and a half times as high as the attainability level; and $100 \%$ equity financing at the high ROI of $8 \%$. These represent a wide range of uncertainties and demonstrate that raising the target PSE to $18 \%$ would result in a feasible technology configuration for most situations.

Cases requiring PSE in excess of $18.5 \%$ to keep the algal production cost at $\$ 192 / \mathrm{t}$ are full equity financing at $9 \% \mathrm{ROI}$, maintenance costs of $20 \%$ of the capital investment cost, and carbon dioxide costs in excess of $\$ 4 /$ thermal scf $\left(\$ 0.14 / \mathrm{m}^{3}\right)$. Rates of return of $9 \%$ (real) and $100 \%$ equity financing are conditions usually associated with risky investments, and these extreme conditions can be avoided by careful technology 
Table 6-19. Mitigating PSE Levels for Attainability Production Case Sensitivities ${ }^{\mathbf{a}}$

\begin{tabular}{|c|c|c|c|c|c|}
\hline \multirow{3}{*}{$\begin{array}{l}\text { Case }^{\mathrm{C}} \\
\text { (1) }\end{array}$} & \multirow{3}{*}{$\begin{array}{l}\text { Parameter } \\
\text { Type } \\
\text { (2) }\end{array}$} & \multirow{3}{*}{$\underset{\text { (3) }}{\text { Parameter }}$} & \multirow{2}{*}{\multicolumn{3}{|c|}{$\frac{\text { Equilibrating Level of PSE }}{\text { Levels of Change }}$}} \\
\hline & & & & & \\
\hline & & & $\begin{array}{l}\text { 1st } \\
(4)\end{array}$ & $\begin{array}{l}\text { 2nd } \\
\text { (5) }\end{array}$ & $\begin{array}{l}3 r d \\
(6)\end{array}$ \\
\hline A. 1 & Resource & Evaporation rate & 16.58 & 16.99 & 18.44 \\
\hline A.2 & Resource & Land cost & 16.34 & 16.66 & 17.03 \\
\hline A. 3 & Resource & Water cost & 17.23 & 17.80 & 18.44 \\
\hline A. 4 & Resource & Carbon dioxide cost & 18.50 & 23.05 & -- \\
\hline A. 5 & Resource & Source water salinity & 16.29 & 16.58 & -- \\
\hline A.6 & Resource & Growing season & 16.82 & 17.63 & 18.44 \\
\hline B.1 & Facility design & BOS cost & 17.15 & 17.50 & 17.96 \\
\hline B.2 & Facility design & Liner cost & 17.56 & 17.96 & 18.40 \\
\hline C.1 & Financial & Maintenance cost & 17.02 & 18.44 & 19.84 \\
\hline C. 2 & Financial & Equity capital return & 16.92 & 18.47 & 20.05 \\
\hline
\end{tabular}

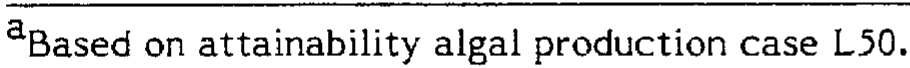

${ }^{b}$ The level of PSE required to maintain algal production cost at $\$ 192 / t$, the level of attainability case L50, given the parameter changes specified for the various cases.

${ }^{\mathrm{C}}$ Cases are defined in Table 6-18.

development including staged demonstrations of individual and integrated components that confirm technological and economic feasibility. In the same vein, well-designed components should keep maintenance costs closer to the typical level of $7 \%-10 \%$ of capital cost. The impact of extreme maintenance costs should alert technology developers to stress equipment reliability at all stages of development. We conclude that two of the three extreme cases (requiring a PSE in excess of 18.5\%) can be viewed as signaling design requirements and not indicative of rigid constraints that threaten feasibility. The extreme carbon dioxide case, however, presents a more formidable constraint.

The cost of $\mathrm{CO}_{2}$ was increased in two increments from the attainability assumption of $\$ 0.10 / \mathrm{m}^{3}$ to first $\$ 0.12$ and then $\$ 0.14 / \mathrm{m}^{3}$, increases of $20 \%$ and $40 \%$, respectively. The $20 \%$ increase was mitigated by increasing the PSE to $18.5 \%$ and decreasing outgassing losses to zero, but the $40 \%$ increase in $\mathrm{CO}_{2}$ cost requires an equilibrating PSE of about $23 \%$. Thus, we looked at other improvements to reach the $\$ 192$ price at a PSE below $20 \%$. At almost $20 \%$ PSE, the production cost still exceeded the attainability requirement of $\$ 192 / \mathrm{t}$ by over $\$ 20 / \mathrm{t}$. We first raised the lipid content to $60 \%$ (lowering 


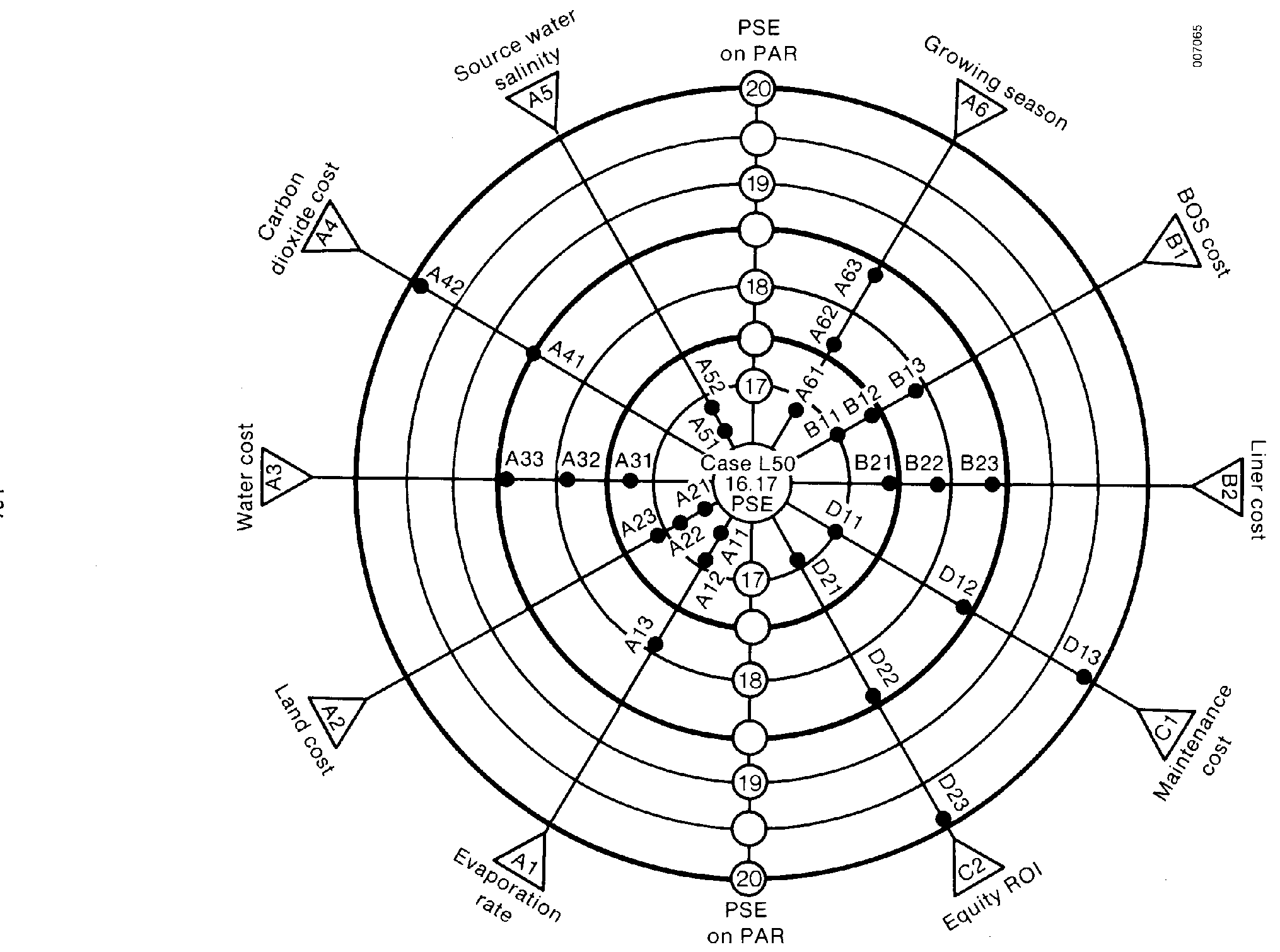

Figure 6-11. Photosynthetic Efficiencies Required to Maintain Fuel Product Costs Equal to the 
cost to $\$ 206 / t)$, then increased the growing season to 320 days $(\$ 200 / t)$, and finally reached $\$ 192 / \mathrm{t}$ by lowering the evaporation rate to about $0.0015 \mathrm{~m} / \mathrm{d}$ (half the attainability rate assumption). Clearly, algal production costs are extremely sensitive to $\mathrm{CO}_{2}$ cost at the attainability configuration.

Carbon is a vital input to the production of any petroleum substitute and probabiy the most constraining factor for any alternative fuel producing technology. That this is the case for microalgae technology should not be viewed as a reason to drop the concept, but rather as an important factor in evaluating the ultimate success of this, versus other alternative fuel technologies. The United States has vast $\mathrm{CO}_{2}$ reserves that may be available at costs compatible with their use in microalgae production. In terms of setting technology performance targets, protecting against high $\mathrm{CO}_{2}$ costs suggests maximizing carbon utilization through developing efficient species and careful design of $\mathrm{CO}_{2}$ input systems. The larger question of the cost and availability of $\mathrm{CO}_{2}$ is a resource question that must be resolved through further evaluation of options and the evolution of $\mathrm{CO}_{2}$-based enhanced oil recovery, a factor outside the realm of influence to microalgae technology development efforts.

\subsubsection{Special Attainability Cases with Low Production Costs}

The previous section examined how resource and economic constraints work to raise the technology performance targets. Equally important are situations where more ideal parameter conditions are encountered since these situations represent the opportunity for a cost-effective system at less stringent levels of PSE and other biological parameters. For example, if conditions exist where a system is cost effective at, say a PSE of only $12 \%$, then the opportunity exists to gain valuable commercial experience that will speed technology development. The goals chosen to guide program activities should be stringent enough to ensure that meeting these goals fulfills the program objective. However, lower interim targets serve to set serial milestones for measuring progress and, if they correspond to special conditions of opportunity, their early adoption will complement the development effort. Thus, we reverse the process of the previous section and look at how more favorable assumptions about certain parameters lower the required PSE.

Sensitivity cases for cost decrements are identified in Table 6-20 and the mitigating PSE levels are illustrated in Figure 6-12. Those factors that had small PSE effects previously, in the case of less favorable levels, also have small impacts in the other direction. Lowering land or water costs by $50 \%$ only decreases production costs by one to three dollars, not enough to offer opportunities for early commercialization, at least for fuel production. The same is true of low salinity source water and $\mathrm{CO}_{2}$ costs of only $\$ 0.08 / \mathrm{m}^{3}$. However, if we bundle together a set of favorable conditions, we do find algal productions costs significantly below the attainability figure of $\$ 192 / \mathrm{t}$.

For example, assuming costs for land $(\$ 500 / \mathrm{ha})$, water $\left(\$ 0.03 / \mathrm{m}^{3}\right)$, carbon dioxide $\left(\$ 0.06 / \mathrm{m}^{3}\right)$, and favorable environmental conditions such as a long growing season (320 days) with higher solar radiation (daily solar constant of $6000 \mathrm{kcal} / \mathrm{m}^{2}$ ), the algal production cost for case $\mathrm{L} 50$ biological target is $\$ 155 / \mathrm{t}$, a $20 \%$ cost reduction that, if processed in a small refinery, might yield a gasoline or ester fuel at under $\$ 1.50 /$ gal. The fuel price projections presented earlier suggest that hydrocarbon fuel prices approach $\$ 1.50 /$ gal about the turn of the century. Thus, early adoption, at least in limited circumstances, is possible if the L50 case biological targets are achieved. But, for the 
Table 6-20. Sensitivity Cases for Attainable Microalgae Production Cost: Uncertainties that Decrease Costs ${ }^{\mathrm{a}}$

\begin{tabular}{|c|c|c|c|c|c|c|c|}
\hline \multirow[b]{2}{*}{ Case } & \multirow[b]{2}{*}{$\begin{array}{c}\text { Parameter } \\
\text { Type }\end{array}$} & \multirow[b]{2}{*}{ Parameter } & \multirow[b]{2}{*}{ Units } & \multirow[b]{2}{*}{$\begin{array}{c}\text { Attaina- } \\
\text { bility }\end{array}$} & \multicolumn{3}{|c|}{ Leveis of Change ${ }^{b}$} \\
\hline & & & & & Ist & 2nd & $3 r d$ \\
\hline $\mathrm{Dl}$ & Resource & Carbon dioxide cost & $\$ / m^{3}$ & 0.10 & 0.08 & 0.06 & -- \\
\hline D2 & Conversion & Fuel value $^{c}$ & $\$ /$ gal & 1.75 & 1.93 & 2.10 & 2.28 \\
\hline D3 & Resource & Growing season & $d / y r$ & 300 & 320 & 340 & -- \\
\hline $\mathrm{D} 4$ & Biological & Lipid content & $\%$ & 50 & 55 & 60 & -- \\
\hline D5 & Resource & Water cost & $\$ / m^{3}$ & 0.067 & 0.045 & 0.034 & - \\
\hline
\end{tabular}

${ }^{\mathrm{a}}$ Cases represent imposition of additional constraints, through higher levels of indicated variables, on the attainability microalgae production concept.

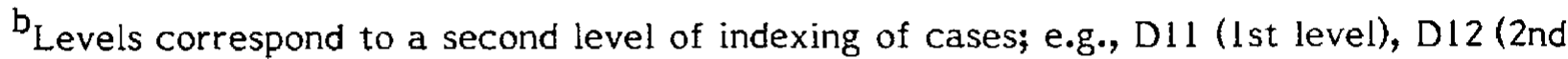
level), D13 (3rd level).

${ }^{\mathrm{C}}$ Gasoline or diesel fuel product cost goal for 2010 .

production of fuel, it appears that the PSE target of at least $16 \%$ with $50 \%$ algal lipid content is a necessary condition for the technology to become economically viable in the first decade of the 21 st century.

\subsubsection{Lipid Sensitivity Cases}

The above sensitivity cases are defined in terms of the PSE increment or decrement required to mitigate changes in parameter assumptions. However, it is of interest to researchers to examine the tradeoff between PSE and lipid content. To evaluate the option between developing productivity (PSE) or lipid content, it is necessary to characterize the trade of functions and then examine the technical feasibility versus the technical requirements.

To assist in the evaluation of alternative research thrusts, we constructed a sensitivity analysis, based on case L50, where PSE and lipid content we revaried against the constraint that the final fuel production cost did not exceed the 2010 cost goal target. Since changing the lipid content changes the value of the raw algal product to the fuel processing step, solution of the tradeoff function requires resolving all steps of the model. That is, as we vary the lipid content from $50 \%$ level of case L50, we must solve for a PSE that holds the final fuel cost constant given the changes in the relative chemical product distribution of the algae.

A single sensitivity case, involving attainability case L50 but for gasoline as the final product, serves to illustrate the tradeoff between PSE and lipid content. For each variation of lipid content, from the $50 \%$ attainability, all changes induced in fuel processing chemistry and cost calculated and a new gasoline production cost is estimated at the $16.17 \%$ PSE level. Then, the PSE is varied until gasoline production costs are returned to the cost goal level and the lipid content PSE pair is plotted. 
The results of this sensitivity analysis, illustrated in Figure 6-13, indicate a relative steep tradeoff function between lipid content and PSE. Of special interest is the section of the curve between $50 \%$ and $60 \%$ lipid content as it indicates that the PSE requirement could be dropped from the L50 attainability level of $16.17 \%$ to about $13 \%$ if $60 \%$ lipids can be achieved. This suggests that careful attention be given to opportunities to increase lipid content, rather that PSE, be seriously evaluated before committing to a specific research strategy. New techniques to induce increased lipid content may yield high returns if PSE reductions can be realized according to Figure 6-13.

The shape of the response or tradeoff curve between lipid content and PSE will vary according to the specific set of parameter assumptions. For fuel products less dependent (than gasoline) on the lipid content for cost effectiveness will demonstrate a less elastic response, and changing the basic input assumptions will shift the curve across the response surface. Specific cases require respecification of the model inputs and are beyond the scope of the analysis. However, we have demonstrated the general concept, and specific evaluations can be conducted to fit the questions of interest in this important area of fuels from microalgae evaluation.

\subsection{Summary}

Although numerous options for the production of fuels from microalgae have been proposed, our analysis indicates that only two qualify for extensive development--gasoline and ester fuel. In developing the comparisons that support this conclusion, we have identified the major areas of microalgae production and processing that require extensive development. Technology success requires developing and testing processes that fully utilize the polar and nonpolar lipids produced by microalgae. Process designs used in these analyses were derived from fragmented, preliminary laboratory data. These results must be substantiated and integrated processes proposed, tested, and refined to be able to evaluate the commercial feasibility from microalgae.

The production of algal feedstocks for processing to gasoline or ester fuel requires algae of high productivity and high lipid content that efficiently utilize saline waters. Species screening and development suggest that algae can achieve required standards taken individually, but algae that can meet the integrated requirements still elude researchers. Effective development of fuels from microalgae technology requires that $R \& D$ be directed toward meeting the integrated standards set out in the analysis. As technology analysts, it is inappropriate for us to dicate how the R\&D effort should proceed to meet these standards. We end our role by noting that alternative approaches to meeting the feasibility targets have been identified, and it is now the task of program managers and scientists to choose the appropriate approach to assure the greatest likelihood of realizing a commercially viable technology. 


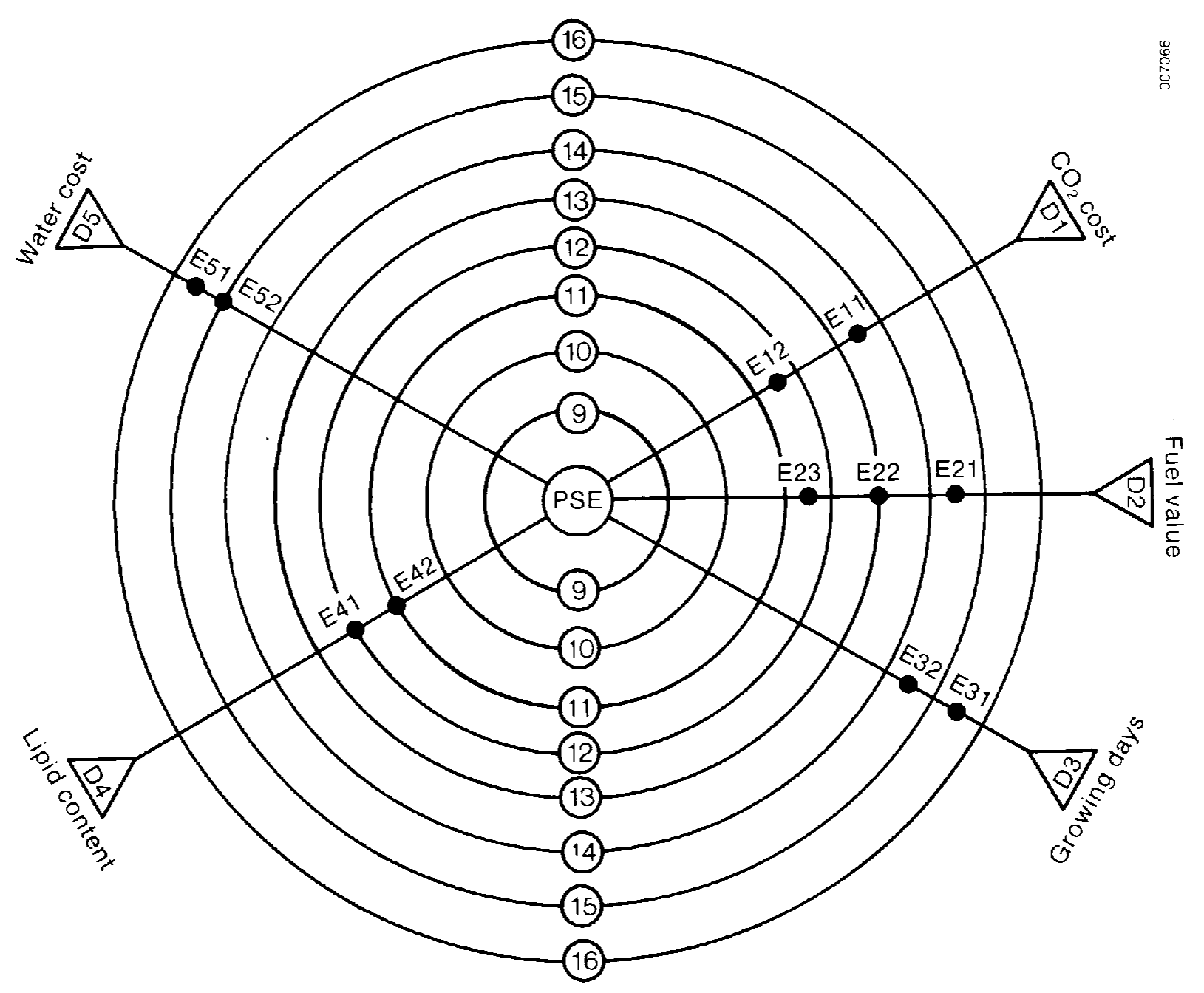

Figure 6-12. Photosynthetic Efficiencies Required to Maintain Fuel Product Costs Equal to the Cost Goals: Uncertainties that Decrease Costs 


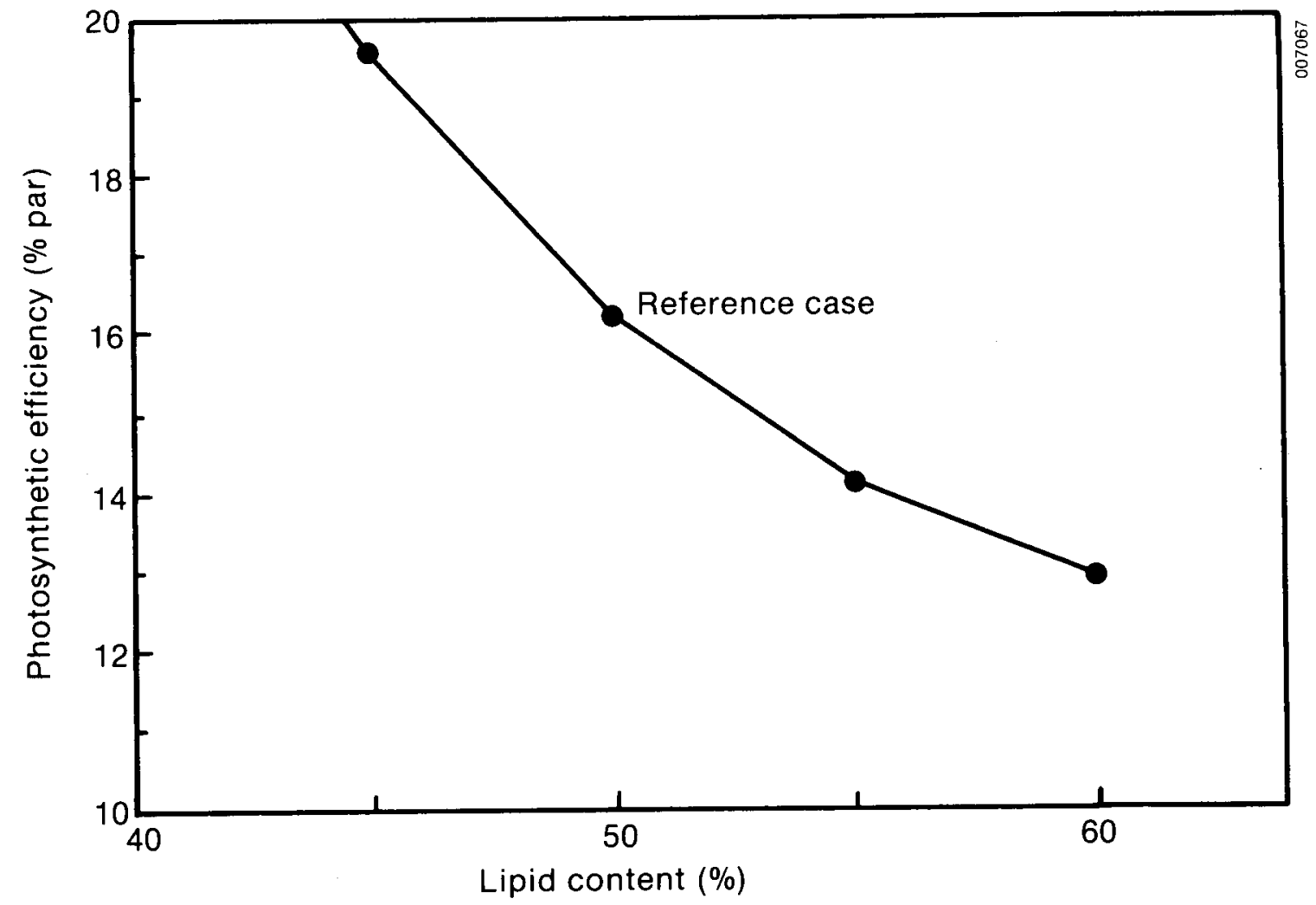

Figure 6-13. Combinations of Lipid Content and Photosynthetic Efficiency that Will Lead to the Production of Cost-Competitive Liquid Fuels 


\subsection{CONCLUSIONS AND RECOMMENDATIONS}

This technology assessment suggests that gasoline and diesel fuels could be produced from mass cultured microalgae at prices that will be competitive with conventional fuels. Aggressive research is needed to fulfill the performance requirements defined by the analysis, but the required improvements are within the bounds of attainability and have been closely approached under controlled conditions. Based on the achievement of these research goals, liquid fuels that are potential direct substitutes for conventional hydrocarbon fuels can be produced from microalgae for $\$ 1.60-\$ 2.00 / g a l$. Conclusions from the analysis and recommendations for future research fit into three categories: fuel products, resource and environmental considerations, and the biological and engineering aspects of fuels from microalgae.

\subsection{Fuel Products from Microalgal Feedstocks}

Analysis of a number of fuel conversion options for microalgal biomass has demonstrated that the promise of microlgae for fuel production is best realized through the utilization of conversion processes based on cellular lipids. The ability to produce lipids that can constitute $50 \%$ or more of the total biomass is a distinguishing characteristic of microalgae and makes them uniquely attractive candidates for conversion to liquid fuels. Conversion of this feedstock to other fuels that fail to take advantage of these unique characteristics, such as fermentation to ethanol or anaerobic digestion to methane, is much less promising.

While microalgal lipids represent the premium energy product, the energy trapped in the other biomass constituents can also be utilized. The cell residue after lipid extraction can be anaerobically digested for the production of methane and carbon dioxide. Analysis has shown that conversion of residues in this manner can contribute to improved process economics.

The two most promising fuel conversion options explored here were transesterification to produce fuels similar to diesel fuels, and catalytic conversion to produce gasoline. However, since only limited performance data are available for these conversion processes, the characterization and optimization of these processes with microalgal lipids must be a subject for continued research. Other unique opportunities for the utilization of microalgal lipids should also be explored.

Research Recommendations. The immediate research priority in microalgal fuel product assessment is a detailed characterization of the fuel properties of microalgal lipids. Attention must be directed toward (1) the identification of techniques by which these lipids can be extracted on a large scale and (2) a detailed description of the characteristics of these lipids as they relate to their suitability as feedstocks for fuel conversion processes. Ultimately, conversion processes specifically tailored to the characteristics of microalgal lipids must be developed, either through the optimization of existing techniques or through the development of innovative conversion technologies. Such research activities will require the production of algal biomass samples on a scale suitable for extraction and fuels characterization. Samples from a number of promising species should be included, since there are strong indications that the characteristics of lipids vary widely between taxa. 


\subsection{Resources and Environmental Considerations}

One of the most attractive features of the microalgal fuels concept is its use of resources for which there are few competing demands. The use of arid lands and saline waters that are not suitable for conventional agriculture will permit the development of microalgae mass culture without detrimental effects on other important plant crops. Adequate suitable land is available in the southwestern United States to provide for a microalgal technology that could make significant contributions to national energy needs.

The availability of saline water will be an important factor in determining the ultimate scale of technology based on this resource. Due to high evaporation rates, water demands for uncovered cultures in this region will be extremely high. While saline aquifers are found throughout the Southwest, the total volume these aquifers can supply on a sustained basis has not been determined. Since very little information is available on the saline water resources of the desert Southwest, the quantification of this resource is a high priority activity.

In addition, we recommend that the concept of microalgal culture for fuels be expanded to include the use of coastal seawater and irrigation runoff water. The scope of the concept should include the consideration of areas where available land with high insolation rates can be identified in coastal regions. The use of irrigation water may also provide an opportunity for an expanded contribution of microalgal fuels to the nation's energy needs. These runoff waters contain large amounts of dissolved inorganics that threaten to pollute clean waters. If these waters can be diverted to microalgal facilities where they contribute to the production of valuable fuels, then microalgal fuel production might be able to contribute another valuable by-product to the economy.

The acquisition of sufficient quantities of carbon dioxide should not impose constraints on the ultimate scale of the microalgal fuels technology. Existing and proposed power plants will produce carbon dioxide in excess of the quantity required for microalgal production. Competing demands from enhanced oil recovery (EOR) are not anticipated, since most oil fields in the area are expected to be depleted by the year 2000 , which is the expected date of emergence of an extensive microalgal mass culture technology for liquid fuel production. If methods were developed for the recovery of carbon dioxide previously injected into oil wells, these large reservoirs could supply additional abundant quantities of low-cost carbon dioxide.

While the supply of carbon dioxide will be more than adequate to support the microalgal technology, this raw material is the largest single contributor to the cost of liquid fuels derived from microalgae. Improvements in the techniques for carbon dioxide recovery from flue gas, or the identification of sources of inexpensive $\mathrm{CO}_{2}$ such as $\mathrm{EOR}$ residuals, would contribute significant additional improvements to the cost competitiveness of microalgal fuels.

No major regional climatic effects are anticipated from the introduction of large uncovered areas of water in this otherwise arid region. The technology will produce two major waste streams--concentrated brines from the blowdown stream and digester sludge from the anaerobic digesters (or similar biomass residues). These waste streams can present either an opportunity for utilization at an economic advantage, or introduce a cost for disposal. Neither possibility has been considered in the economic analysis.

Research Recommendations. Both the microalgal production model and the advice of experts in the field have emphasized the significance of saline groundwater as a potential 
limiting resource for the development of a large-scale microalgal technology in the desert Southwest. To assess the potential scale of this technology, it is critical that accurate estimates of the sustainable yields of the saline aquifers in the area be determined. Factors of interest are total aquifer volume, recharge rates, and potential changes in water composition with prolonged pumping. In addition, the effects of extensive pumping of saline aquifers on potable water aquifers in the same region will have to be assessed. Beyond an assessment of the saline groundwater resource, we also recommend an assessment of the magnitude of the possible contribution of coastal seawater and agricultural runoff to a microalgal fuels technology.

\subsection{Biological and Engineering Aspects of Feedstock Production}

The major issues to be resolved in mass culture technology are biological. In order for this technology to become cost competitive, the biological productivity of these systems must be improved. The production analysis indicates that photosynthetic efficiencies on the order of $16 \%$ must be attained, and that about $50 \%$ of the biomass produced must be in the form of lipids. If higher lipid contents were achieved, lower photosynthetic efficiency would be acceptable, and vice versa.

Lipid content of $50 \%$ or more can be obtained routinely in the laboratory, but program activities to date have only achieved photosynthetic efficiencies on the order of 10\%-13\%. Nevertheless, these photosynthetic efficiencies represent significant improvements over those previously obtained, and can be attributed to improvements in both the selection of species for cultivation and in culture management strategy. With appropriate research in these areas, continued progress can be expected. It is critical, however, that both biomass and lipid productivity requirements be achieved simultaneously at sustained rates. That is, the allocation of carbon between lipids and other cell constituents must be shifted in favor of the lipids without excessive loss of photosynthetic efficiency.

In addition to displaying improved productivity, species must demonstrate environmental tolerance characteristics that make them suitable for outdoor culture in arid regions. The productivity analysis has identified species salinity tolerance as a particularly significant aspect of environmental tolerance, since the operation of cultures at high salinities is necessary to control water requirements through a minimization of water consumption for blowdown. Additional reduction in water and nutrient use requires mechanisms for control of evaporation and nutrient outgassing. Conventional plastic covers are too expensive, so new techniques are required to improve resource productivity.

The basic engineering needs for microalgal culture have been identified and form the basis of a significant portion of the production analysis. Considerable additional engineering research and development will be required, but such activities cannot be undertaken until the basic requirements of suitable microalgal species can be established. The harvesting of microalgae, however, is an engineering issue that will require continued attention. The aspects of microalgae that affect their harvestability have been characterized adequately to permit the continued exploration of different harvesting options. In addition, an understanding of the performance of various harvesting processes will help to determine desirable species characteristics for harvestability. 
Research Recommendations. The identification or development of microalgal strains that will meet the performance criteria of high productivity, high lipid content, and wide ranges of environmental tolerance is the single most critical research requirement for the economic viability of a microalgal fuels technology. The ability to meet these requirements must first be established in closely controlled experimental cultures, then confirmed under conditions that more closely approximate outdoor mass culture conditions. The ultimate success of the fuels-from-microalgae concept is critically dependent on the rate and degree of species improvement.

Continued investigation of harvesting techniques is recommended, as is an emphasis on a characterization of the performance of different species in various harvesting systems. The identification of species characteristics that determine their harvestability is a critical input to the selection and development of microalgal species for use in outdoor production systems: a strong interaction between species development and harvester engineering activities will be critical to the development of species that meet performance criteria and yet can be harvested economically. Finaliy, it is recommended that mechanisms for control of evaporation and nutrient outgassing be explored to evaluate the potential for minimizing the cost of these resources. 


\subsection{REFERENCES}

Adams, A. H., W. E. Luttges, M. Mullen, M. C. Rand, J. R. Peters, B. J. Schorer, B. J., and M. C. Ziemke, 1981, Study on Production, Marketing and Utilization of Degummed Soybean Oil as a Diesel Fuel Extender, University of Alabama at Huntsville.

Aiba, S., and T. Ogawa, 1983, "On the Criticism of Measurements in Phososynthetic Efficiency," Biotech. Bioeng. Vol. 25, pp. 2775-2779.

Anada, H. M., D. Fraser, D. F. King, A. P. Seskus, and J. T. Sears, 1983, "Economics of By-Product $\mathrm{CO}_{2}$ Recovery and Transportation for EOR," Energy Progress, Vol. 3, No. 4, p. 233.

Ashare, E, and E. H. Wilson, 1979, Analysis of Digester Design Concepts, USDOE COO2991-42, Cambridge, MA: Dynatech R. and D. Laboratory.

Barclay, W., 1984a, unpublished data.

Barclay, W., 1984b, personal communication, 28 November.

Barclay, W., and R. McIntosh, eds., 1986, Algal Biomass Technologies: An Interdisciplinary Perspective, Berlin Stuttgart, Germany, J. Cramer.

Benemann, J. R., et al., 1977, Species Control in Large Scale Microalgae Biomass Production," Final Report, Sanitary Engineering Research Laboratory, University of California at Berkeley, SERL Report 77-5, USDOE TID27702.

Benemann, J. R., et al., 1982, Microalgae as a Source of Liquid Fuels, prepared by Enbio, Inc., for the U.S. Department of Energy's Office of Energy Research, Contract DEACOS 81 ER 30014.

Bonin, D. J., S. Y. Maestrini, and J. W. Leftly, 1981, "Some Processes and Physical Factors that Affect the Ability of Individual Species of Algae to Compete for Nutrient Partition," Can. Bull. Fish Aquatic Sci., Vol. 210, pp. 292-309.

Burlew, J. S., ed., 1953, Algal Culture: From Laboratory to Pilot Plant, Washington, DC: Carnegie Institution of Washington, Publication No. 600, 351 pp.

Caperon, J., and J. Meyer, 1972, "Nitrogen-Limited Growth of Marine Phytoplankton--I. Changes in Population Characteristics with Steady-State Growth Rate," Deep-Sea Res., Vol. 19, p. 601 .

Caperon, J., and D. F. Smith, 1978, "Photosynthetic Rates of Marine Algae as a Function of Inorganic Carbon Concentration," Limnol. Oceanogr., Vol. 23, p. 704.

Chemical Marketing Reporter, 23 July 1984.

Cheney, D., 1986, "Genetic Engineering in Seaweeds: Applications and Current Status," in Algal Biomass Technologies, W. Barclay and R. P. McIntosh, eds., Beihefte zur Nova Hedwigia, Vol. 83, pp. 22-29. 
Chin, L.-Y., and A. J. Engel, 1981, "Hydrocarbon Feedstocks from Algae Hydrogenation," Biotech. and Bioeng. Symp., No. 11, pp. 171-86.

Clark, S. J., L. Wagner, M. D., and P. G. Piennar, 1984, "Methyl and Ethyl Soybean Esters as Renewable Fuels for Diesel Engines," J. Am. Oil Chem. Soc., Vol. 61 (10), p. 1632.

Data Resources, Inc., 1984, The Data Resources U. S. Long Term Review, NY: McGrawHill.

Doane, J. W., R. P. O'Toole, R. G. Chamberlain, P. B. Bos, and P. D. Maycock, 1976, The Cost of Energy from Utility Owned Solar Electric Systems, ERDA/JPL-1012-76/3, Pasadena, CA: Jet Propulsion Laboratory.

Dodd, J., 1984, personal communication, 12 November.

Dubinsky Z., T. Berner, and S. Aaronson, "Potential of Large Scale Algal Culture for Biomass and Lipid Production in Arid Lands," Biotech. and Bioeng. Symp. No. 8, p. 51.

Edison Electric Institute, 1982, Statistical Yearbook of the Electric Utility Industry.

Energy Information Agency, 1983, Typical Electric Bills Report.

Feinberg D. A., 1984, Fuel Options from Microalgae with Representative Chemical Compositions, SERI/TR-231-2427, Golden, CO: Solar Energy Research Institute.

Feth, J. H., et al., 1965, Preliminary Map of the Coterminous United States Showing Depth to and Quality of Shallowest Ground Water Containing More than $1000 \mathrm{ppm}$ Dissolved Solids, Hydrologic Investigations Atlas, HA-199, U. S. Geological Survey.

Freedman, B., and E. H. Pryde, 1982, "Fatty Esters from Vegetable Oils for Use as a Diesel Fuels," Proceedings of the International Conference on Plant and Vegetable Oils as Fuels, American Society of Agricultural Engineers, p. 117.

Goldman, J. C., 1978, Fuels from Solar Energy: Photosynthetic Systems-State of the Art and Potential for Energy Production, USDOE Report No. COO-4151-2.

Hesketh, H. E., 1979, Air Pollution Control, Ann Arbor, MI: Ann Arbor Science Publishers, Inc.

Hill, A. M., 1984, Algal Production and Economic Model Documentation, draft report available from the Aquatic Species Program, Solar Energy Research Institute, Golden, CO.

Hill, A. M., and D. A. Feinberg, 1984, Fuel From Microalgae Lipid Products, SERI/TR231-2348, Golden, CO: Solar Energy Research Institute.

Huntsman, S. A., and W. G. Sunda, 1980, "The Role of Trace Metals in Regulating Phytoplankton Growth," in The Physiological Ecology of Phytoplankton, I. Morris, ed., Berkeley: Univ. of Calif. Press, pp. 285-328.

Huguenin J., 1984, personal communication, 6 September. 
International Trade Commission, 1981, Synthetic Organic Chemicals, U. S. Production and Sales, 1980, Washington, D.C.

International Trade Commission, 1982, Synthetic Organic Chemicals, U. S. Production and Sales, 1981, Washington, D.C.

Jaycor, 1983, Biological and Engineering Parameters of Algal Mass Culture Systems, report on subcontract XX-02123-01 to the Solar Energy Research Institute.

Kusy, P. F., 1982, "Transesterification of Vegetable Oils as Fuels," Proceedings of the International Conference of Plant and Vegetable Oils as Fuels, American Society of Agricultural Engineers, p. 127.

Kern, J. C., 1980, "Glycerol," in Encyclopedia of Chemical Technology, 3rd ed., Kirk and Othmer, eds., NY: Wiley-Interscience.

Lane J. C., 1980, "Gasoline and Other Motor Fuels," in Encyclopedia of Chemical Technology, 3rd ed., Kirk and Othmer, eds., NY: Wiley Interscience, Vol. 11, p. 652.

Laws, E. A., K. L. Terry, J. Wickman, and M. S. Chalup, 1983, "Preliminary Results from a Simple Algal Production System Designed to Utilize the Flashing Light Effect," Biotech. Bioeng., Vo!. 25, p. 2319.

Laws, E. A., 1984, Research Development of Shallow Algal Mass Culture Systems for the Production of Oils, SERI/STR-231-2496, Subcontract Report, Golden, CO: Solar Energy Research Institute.

Maxwell, E. L., A. G. Folger, and S. E. Hogg, 1984, Resource Evaluation and Site Selection for Microalgae Production Systems, SERI/TR-215-2484, Golden, CO: Solar Energy Research Institute.

McCallum, P. W., T. J. Timbario, R. C. Bechtold, and E. E. Ecklund, 1982, "Alcohol Fuels for Highway Vehicles," Chemical Engineering Progress, Vol. 78 (8), p. 52.

McLachlan, J., 1964, "Some Considerations on the Growth of Marine Algae in Artificial Media," Can. J. Microbiol., Vol. 10, p. 769.

Meegan, M. K., ed., 1977, The Kline Guide to the Chemical Industry, Third Ed., Fairfield, NJ: Charles H. Kline \& Co., Inc.

Meier, R. L., 1955, "Biological Cycles in the Tranformation of Solar Energy into Useful Fuels," in Solar Energy Research, F. Daniels and J. A. Duffie, eds., Madison: Univ. Wisconsin Press, p. 179.

Miyachi, S., M. Tsuzuki, and Y. Yagawa, 1985, "Carbonic Anhydrase in Various Microalgae," in Inorganic Uptake by Aquatic Photosynthetic Organisms," W. J. Lucas and J. A. Berry, eds., American Society of Plant Physiologists, pp. 145-154.

Mohn F. H., 1980, "Experiences and Strategies in the Recovery of Biomass from Mass Cultures of Microalgae," in Algal Biomass, G. Shelef and C. J. Soeder, eds., NY: Elsevier/North Holland Biomedical Press, p. 547. 
National Petroleum Council, 1984, "Enhanced Oil Recovery," National Petroleum Council's Committee on Enhanced Oil Recovery, also, National Petroleum Council's "Enhanced Oil Recovery" report, 1976.

Nelson, S. H., 1982, An Investigation of the Availability of Carbon Dioxide for the Production of Microalgal Lipids in the Southwest, ANL/CNSV-42, Argonne, IL: Argonne National Laboratory.

Parsons et al., 1961, "On the Pigment Composition of Eleven Species of Marine Phytoplanktons," J. Fish. Res. Bd. Can., Vol. 18, p. 1017.

Perry R. H., and D. W. Green, eds., 1984, Perry's Chemical Engineers Handbook, 6th ed., NY: McGraw-Hill.

Petzet, G. A., 1983, "U. S. Industry Eyes Buildup in Oil Output via $\mathrm{CO}_{2}$ Floods," Oil \& Gas Journal, Jan. 3, p. 39.

Pryde E. H., 1982, "Vegetable Oil Fuel Standards," in Proceedings of the International Conference on Plant \& Vegetable Oils as Fuels, p. 101.

Pryde, E. H., 1984, personal communication, 14 November.

Radmer, R., and B. Kok, 1977, "Photosynthesis: Limited Yields, Unlimited Dreams," Bioscience, Vol. 27, p. 599.

Raphael Katzen Associates, 1980, "Grain Motor Fuel Technical and Economic Assessment Study," in Large and Small Scale Ethyl Alcohol Manufacturing Processes from Agricultural Raw Materials, J. K. Paul, ed., Park Ridge, NJ: Noyes Data Corp.

Reynolds A., 1982, Projected Costs of Electricity from Nuclear and Coal-fired Power Plants, DOE/EIA-0356/2, U. S. Energy Information Administration.

Ryan, T. W., III, L. G. Dodge, and T. J. Callahan, 1984, "The Effects of Vegetable Oil Properties on Injection and Combustion in two Different Diesel Engines," J. Am. Oil Chem. Soc.,Vol. 61 (10), p. 1610.

Saga, N., M. Bolne-Fuller, and A. Gibor, 1986, "Protoplasts from Seaweeds: Production and Fusion," in Algal Biomass Technologies, W. Barclay and R. P. McIntosh, eds., Beihefte zur Nova Hedwigia, Vol. 83, pp. 37-43.

Samson R., and A. Leduy, 1983, "Influence of Mechanical and Thermochemical Pretreatments on Anaerobic Digestion of Spirulina maxima Algal Biomass," Biotechnology Letters, Vol. $5(10)$, p. 671 .

Shelef, G., and C. J. Soeder, eds., 1980, Algae Biomass, Production and Use, Amsterdam: Elsevier/North-Holland Biomedical Press, 852 pp.

Sherbet, G. V., 1978, The Biophysical Characterization of the Cell Surface, NY: Academic Press, 298 pp.

Shifrin, M. S. 1980, Phytoplankton Lipids: Environmental Influences on Production and Possible Commercial Applications, Ph. D. Thesis, Massachusetts Institute of Technology, Boston, MA. 
Shreve, R. N., and J. A. Brink, Jr., 1977, Chemical Process Industries, 4th ed., NY: McGraw-Hill.

Stumm, W., and J. J. Morgan, 1970, Aquatic Chemistry, NY: Wiley-Interscience.

Tahir, A. B., H. M. Lapp, and L. C. Buchanan, 1982, "Sunflower Oil as a Fuel for Compression Ignition Engines," in Proceedings of the International Conference on Plant and Vegetable Oils as Fuels, American Society of Agricultural Engineers, p. 82.

Technical Insights, Inc., 1980, Biomass Process Handbook, NJ: Fort Lee.

Terry, K. L., 1982, "Nitrate Uptake and Assimilation in Thalassiosira weissflogii and Phaeodactylum Tricornutum: Interactions with Photosynthesis and with the Uptake of Other Ions," Mar. Biol., Vol. 69, p. 21.

Terry, K. L., and L. P. Raymond, 1985, "System Design for the Autotrophic Production of Microalgae," Enzyme and Microbial Technology, Vol. 7, pp. 474-487.

Terry, K. L., J. Hirata, and E. A. Laws, 1985, "Light-Limited, Nitrogen-Limited, and Phosphorus-Limited Growth of Phaeodactylum tricornutum Bohlin strain TFX-1: Chemical Composition, Carbon Partitioning, and the Diel Periodicity of Physiological Processes," J. Exp. Mar. Biol. Ecol., Vol. 86, No. 1, pp. 85-100.

Tornabene, T. G., A. Ben-Amotz, S. Raziuddin, and J. Hubbard, 1983, "Chemical Profiles of Microalgae with Emphasis on Lipids," Final Report for SERI Subcontract No. XK-202149-1 (portions also published in Screening for Lipid Yielding Microalgae, Final Subcontract Report SERI/STR-231-2207), Golden, CO: Solar Energy Research Institute.

Ukeles, R., 1976, "Cultivation of Plants: Unicellular Plants," in Marine Ecology, Vol. HI, Cultivation, NY: John Wiley and Sons, pp. 367-466.

Vigon, B. W., M. F. Arthur, L. G. Taft, C. K. Wagner, E. S. Lipinsky, J. N. Litchfield, C. McCandlish, and R. Clark, 1982, "Resource Assessment for Microalgal/Emergent Aquatic Biomass Systems in the Arid Southwest," draft report to Solar Energy Research Institute by Battelle Columbus Laboratories, Columbus, $\mathrm{OH}$.

Voltz, S. E., et al., 1976, Development Studies on Conversion of Methanol \& Related Oxgenates to Gasoline, Energy Research and Development Administration Report FE-1773-25, Paulsboro, NJ: Mobil Research and Development Corp.

Wagener, K, 1981, "Mariculture on Land-A System for Biofuel Farming in Coastal Deserts," Biomass, Vol. 1, p. 145.

Weisz, P. B., et al., 1979, "Catalytic Production of High-Grade Fuel (Gasoline) from Biomass Compounds by Shape-Selective Catalysis," Science, Vol. 206, p. 57.

Zimmerman, F. W., and C. W. Perry, 1979, Naturally Occurring Carbon Dioxide Sources in the United States, FE-2024-38, Bellaire, TX: Gulf Universities Research Consortium. 


\section{APPENDIX A}

This appendix presents the Microalgae Production Model in Fortran 5. The program listing is provided for those readers interested in determining specific values as they were presented in this report. The program listing is provided as a reference and should not be considered as a executable program listing as it is presented. Comments and suggestions for improvement to this model are encouraged by the authors of this report. Should the public be interested in running this program, copies of this program are available on PC floppy disks by calling or writing:

Donna A. Johnson

Aquatic Species Program

Solar Energy Research Institute

1617 Cole Blvd.

Golden, CO 80401

(303) $231-1472$ 
Page 1

D Line\# 17 Microsoft

FORTRAN77 V3.20 02/84

PROGRAM TMICRO

DIMENSION AA(86),HCONC(4), UHARV(4), HCOST(4), $\operatorname{HEXP(4)}$

REAL

NUT,IU, NORM, LENGTH,MIXVEL,K WHCOST, LIPCON, NITCOST, NITWT, $\& \mathrm{~K}, \mathrm{KC}, \mathrm{KD}, \mathrm{KP}, \mathrm{MNT}, \mathrm{N}, \mathrm{M}, \mathrm{LAND}, \mathrm{NDCI}$, MODULE,MODSIZE, NUTC, \& NUTN, NUTP, NUTK, LCOST,MIXCC,NW,KW, NAR,KAR, NAG,ICON,NPROT, \&LEC,IEC,KCON,NCON,LINE, NHARV,NHARVB

CHARACTER*60 ATEXT(86)

EQUIVALENCE (AA(1), RESOUR),(AA(2),ERATE),(AA(3),SALIN), $\&(\mathrm{AA}(4), \mathrm{NW}),(\mathrm{AA}(5), \mathrm{PW}),(\mathrm{AA}(6), \mathrm{KW}),(\mathrm{AA}(7), \mathrm{CW}),(\mathrm{AA}(8)$, \&LAND),(AA(9),KWHCOST),(AA(10),WATCOST),(AA(11),CF), \&(AA(12),DSC),(AA(13), NITCOST),(AA(14),COKM),(AA(15), " \&POTCOST),(AA(16),PHOCOST),(AA(17),FACIL),(AA(18), \&FACSIZ),(AA(19),EFFCUL),(AA(20),EFFOP),(AA(21),MODULE), \&(AA(22),X),(AA(23),Y),(AA(24),CHAN),(AA(25), ROUGH), \&(AA(26),DTIME),(AA(27),DEPTH),(AA(28),CAR),(AA(29),NAR), \&(AA(30),PAR),(AA(\# 1),KAR),(AA(32),CAG),(AA(33),NAG), \&(AA(34),MIXVEL),(AA(35), EFFPUMP),(AA(36),EFFDRIV), $\&(\mathrm{AA}(37), \mathrm{H}),(\mathrm{AA}(38), \mathrm{ALC}),(\mathrm{AA}(39), \mathrm{EFFHARV}),(\mathrm{AA}(40), \mathrm{EH})$, \&(AA(41),BIOL),(AA(42),EFFPHOT),(AA(43),LIPCON), $\&(\mathrm{AA}(44), \mathrm{CARBCON}),(\mathrm{AA}(45), \mathrm{ACON}),(\mathrm{AA}(46), \mathrm{ICON}),(\mathrm{AA}(47), \mathrm{UMAX})$, \&(AA(48), NPROT),(AA(49), RPROT),(AA(50),QN),(AA(51),LEC), $\&(\mathrm{AA}(52), \mathrm{CEC}),(\mathrm{AA}(53), \mathrm{PEC}),(\mathrm{AA}(54), \mathrm{IEC}),(\mathrm{AA}(55), \mathrm{CCON}),(\mathrm{AA}$ $\&(56), \mathrm{PCON}),(\mathrm{AA}(57), \mathrm{KCON}),(\mathrm{AA}(58), \mathrm{DENSITY}),(\mathrm{AA}(59), \mathrm{SALTOL})$ \&,(AA $(60), F I N A N),(A A(61), X B),(\mathrm{AA}(62), Y T),(\mathrm{AA}(63)$, \&YP),(AA(64),YCO),(AA(65),N),(AA(66),M),(AA(67),B1) EQUIVALENCE (AA(68),B2),(AA(69),T),(AA(70), ALPHA), \&(AA(71),DV),(AA(72),CV),(AA(73),PV),(AA(74),KD), $\&(\mathrm{AA}(75), \mathrm{KC}),(\mathrm{AA}(76), \mathrm{KP}),(\mathrm{AA}(77), \mathrm{G}),(\mathrm{AA}(78), \mathrm{GC}),(\mathrm{AA}(79)$, $\& \mathrm{GO}),(\mathrm{AA}(80), \mathrm{GM}),(\mathrm{AA}(81), \mathrm{K}),(\mathrm{AA}(82), \mathrm{CRF}),(\mathrm{AA}(83), \mathrm{FCR})$ \&,(AA(84),XBBLS),(AA(85),XPRO),(AA(86),XCAR)

DATA HCONC/10.,10.,150.,70./

DATA UHARV $/ .2, .1,1.2, .88 /$

DATA HCOST $/ 123200 ., 33000 ., 625000,184800 . /$

DATA HEXP $/ .8, .8, .8, .87 /$

DATA NITWT/1.21/CARWT/3.67/POTWT/1.20/PHOWT/2.29/

DATA VISC/.00131/DENS/1011.0/

OPEN $(5$, FILE $=$ TMICIN.DAT')

OPEN $\left(6, F I L E=' T M I C O U T . D A T ', S T A T U S=N E W^{\prime}\right)$

C

OPEN $\left(8, F I L E=T E C O I N . D A T^{\prime}\right)$ 
C VARIABLE DEFINITIONS

C

C EFFHARV = HARVESTER SOLIDS REMOVAL EFFICIENCY

C EH = SOLIDS CONC. AFTER HARVEST (SYSTEM REQUIREMENT)

C ATNET = THEORETICAL, LIGHT LIMITED NET PRODUCTION YIELD, MT/YR

C ANET = SYSTEM DEPENDANT NET YIELD, MT/YR

C FACSIZ = SIZE OF FACILITY, HECTARES

C MODULE = NUMBER OF MODULES Page 2

FORTRAN77 V3.20 02/84

C MODSIZE = SIZE OF MODULES, HA

C EFFCUL = EFFECTIVE CULTURE AREA, \%

C EFFOP = CULTURE AREA DOWN FOR MAINTENANCE, $\%$

C TPI =TOTAL PLANT INVESTMENT, \$

C CIPOND $=$ CAPITAL INVESTMENT FOR POND, \$

C CIHARV = CAPITAL INVESTMENT FOR HARVESTOR, \$

C ENGFEE = ENGINEERING AND FEE COSTS, \$

C CONTG $=$ CONTINGENCY FEES, $\$$

C $\quad \mathrm{CI}=$ TOTAL DEPRECIABLE INVESTMENT, \$

C YB,BASE YEAR FOR CONSTANT DOLLARS

C YT, YEAR FOR INVESTMENT OUTLAY

C YP, YEAR FOR COST INFORMATION

C YCO, YEAR OF FIRST COMMERCIAL OPERATION

C N, SYSTEM OPERATING LIFETIME (BOOK LIFE)

C M, TAX LIFE FOR DEPRECIATION,YEARS

C B1, ANNUAL 'OTHER TAXES', AS A FRACTION OF CI

C B2, ANNUAL INSURANCE PREMIUMS, AS A FRACTION OF CI

C T, EFFECTIVE INCOME TAX RATE

C ALPHA, INVESTEMENT TAX CREDIT

C DV, RATIO OF DEBT TO TOTAL CAPITALIZATION

C CV, RATIO OF COMMON STOCK TO TOTAL CAPITALIZATION

C PV, RATIO OF PREFERRED STOCK TO TOTAL CAPITALIZATION

C KD, ANNUAL RATE OF RETURN ON DEBT

C KC, ANNUAL RATE OF RETURN ON COMMON STOCK

C KP, ANNUAL RATE OF RETURN ON PREFERRED STOCK

C G, GENERAL RATE OF INFLATION

C GC, ESCALATION RATE FOR CAPITAL COSTS

C GO, ESCALATION RATE FOR OPERATING COSTS

C GM, ESCALATION RATE FOR MAINTANCE COSTS

C K, COST OF CAPITAL (OPTIONAL)

C CRF,CAPITAL RECOVERY FACTOR(OPTIONAL)

C FCR, FIXED CHARGE RATE (OPTIONAL)

C LIPCON, FRACTIONAL LIPID CONTENT OF ALGAE

C CARBCON, FRACTIONAL CARBOHYDRATE CONYENT OF ALGAE

C ICON $=$ FRACTIONAL NON-SPECIFIED CONTENT OF ALGAE

C BBLS, NET LIPID OIL PRODUCED, BBLS/YR

C NUT = NUTRIENTS, \$

C $\quad$ DL $=$ DIRECT LABOR, \$

C OH = OVERHEAD, \$

C HCONC(TH) $=$ CONCENTRATION RATIO FOR HARVESTER TYPE IH

C HEXP(TH $)=$ EXPONENT FOR CAPITAL COST OF HARVESTOR

C EFFPUMP $=$ MIXING PUMP EFFICIENCY

C EFFDRIVE = MIXING DRIVE EFFICIENCY 
C DTIME $=$ DETENTION (OR DOUBLING) TIME, DAYS

C DEPTH $=$ TROUGH DEPTH, METERS

C $\mathrm{X}=$ MODULE WIDTH, NORMALIZED

C $Y=$ MODULE LENGTH, NORMALIZED

C LENGTH = MODULE LENGTH, METERS

C NORM = NORMALIZING FACTOR FOR MODULE DIMENSIONS

C CHAN = DESIRED CHANNEL WIDTH (METERS)

C MIXVEL = MIXING VELOCITY, METERS/SEC

C KWHCOST = COST OF ENERGY, \$/KWH

C $\quad$ VOL = MODULE VOLUME, M3

C FLRATE = REQUIREDFLOW RATE INTO HARVESTER, CUBIC METERS/MIN

C QHARV = MASS FLOW RATE INTO HARVESTER, GPM/MODULE

C QRECYC = MASS FLOW RATE OF RECYCLED MEDIA, GPM/MODULE

C QMAKEUP = MASS FLOW RATE OF MAKEUP WATER, GPM/MODULE

C ERATE = EVAPORATION RATE, CM/YR

C QEVAP = EVAPORATION LOSSES, GPM/MODULE Page 3

D Line\# 17

FORTRAN77 V3.20 02/84

Microsoft

C UPHARV = PUMPING POWER REQUIREMENT INTO HARVESTER, HP

C UPRECYC = PUMPING POWER REQUIREMENT FOR RECYCLED MEDIA, HP

C UPMUP = PUMPING POWER REQUIREMENT FOR MAKE UP WATER, HP

C UPTOT = TOTAL PUMPING POWER REQUIREMENT, HP

C UPKWH = UTILITY REQUIREMENT FOR TOTAL PUMPING, KWH/YR

C HL = HEAD LOSS FOR MIXING, METERS

C UMIX = MIXING POWER REQUIREMENT, HP

C UMKWH = UTILITY REQUIREMENT FOR MIXING,KWH/YR

C UHARV(IH) = HARVESTOR TYPE IH ENERGY REQUIREMENT, KWH/M3

C UHKWH = UTILITY REQUIREMENT FOR HARVESTING, KWH/YR

C UTOT = TOTAL ENERGY REQUIREMENT FOR PUMPING, MIXING, AND HARVESTING,

C UT = COST OF ENERGY FOR PUMPING, MIXING, AND HARVESTING, $\$ / Y R$

C MNT = OPERATION AND MAINTENENCE, $\$$

C OP = TOTAL VARIABLE COSTS, $\$$

C RH $=$ HYDRAULIC DIAMETER, METERS

C RE = REY NOLDS NUMBER

C RF $=$ RFACTOR

C $Q F=$ INTERIM

C OT = INTERIM

C DCI = DEPRECIABLE CAPITAL INVESTMENT

C NDCI = NON DEPRECIABLE CAPITAL INVESTMENT

C CF = CAPACITY FACTOR, DAYS/YR

C DSC = DAILY SOLAR CONSTANT, KCAL/SQ M-DAY

C EFFPHOT = PHOTOSYNTHETIC EFFICIENCY, \%

C $H=I H$, INDEX FOR HARVESTOR TYPE (1 THRU 3)

C ALC INDEX FOR SECOND HARVESTOR SYSTEM

C NITCOST, PRICE FOR NITROGEN SOURCE, NH3, \$/MT

C CARCOST, PRICE FOR CARBON SOURCE, CO2, \$/MT

C POTCOST, PRICE FOR POTASSIUM SOURCE, KO2, \$/MT

C PHOCOST, PRICE FOR PHOSPHORUS SOURCE, CAP205, \$/MT

C NUTC =TOTAL COST FOR CARBON, \$/YR

C NUTN $=$ TOTAL COST FOR NITROGEN, \$/YR

C $\quad$ NUTK $=$ TOTAL COST FOR POTASSIUM, \$ $/$ YR 


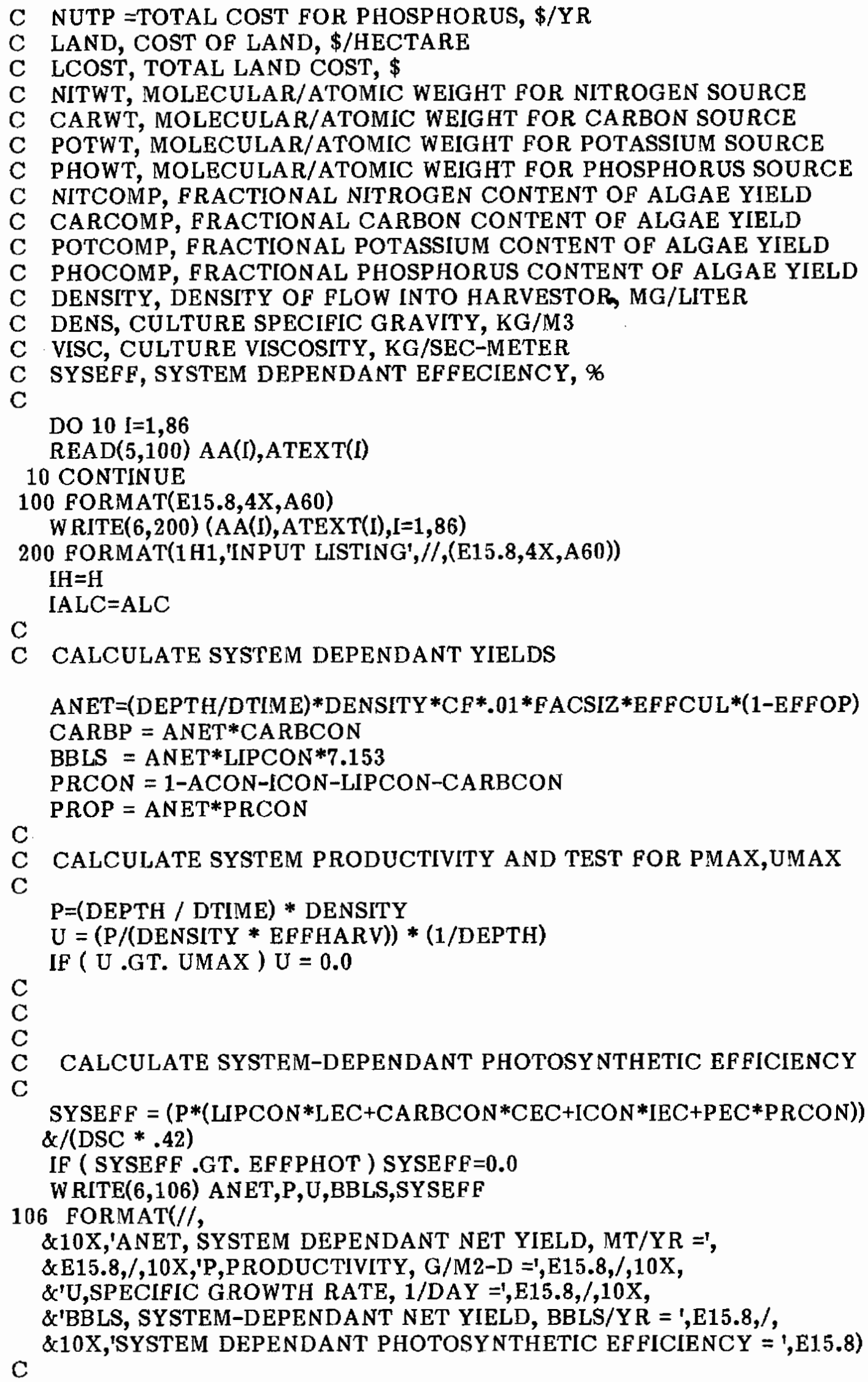


C

CALCULATE CHANNEL DIMENSIONS,EFFECTIVE VOLUME AND FLOW RATES

MODSIZE $=($ EFFCUL $*$ FACSIZ $) /$ MODULE

WIDTH $=$ SQRT $((M O D S I Z E * 10000) / Y)$

LENGTH $=($ MODSIZE $* 10000) /$ WIDTH

W RITE $(6,101)$ WIDTH,LENGTH,MODULE,MODSIZE

101 FORMAT(10X,'WIDTH,METERS=',F10.2,5X,'LENGTH,METERS=',F10.2,/

$\mathrm{C}$

\&10X,'NUMBER OF MODULES = ',F8.2,5X,'MODULE SIZE,HA = ',F8.2)

C CALCULATE WATER BALANCE FOR FACILITY (M3/MIN)(.000694=1/HR*MIN)

$\mathrm{C}$

QMAKEUP $=(($ SALTOL*ERATE $) /($ SALTOL-SALIN $)) *$ FACSIZ*

$\& 10000 *$ EFFCUL*(1-EFFOP)*.0006944

WATLOSS $=(($ SALIN *ERATE $) /($ SALTOL-SALIN $)) *$ F ACSIZ*

$\& 10000 *$ EFFCUL*(1-EFFOP)*.0006944

WATPRO $=((P *$ FACSIZ* $10000 *$ EFFCUL*(1-EFFOP $) * 0.0006944)-$

$\&($ W ATLOSS*DENSITY*(1-EFFHARV)))/((EH*10000)-

\&DENSITY*(1-EFFHARV))

WATDISP $=$ WATLOSS - WATPRO

QHARV $=(($ P $/($ DENSITY*EFFHARV $))$

\&*FACSIZ*10000*EFFCUL*(1-EFFOP)*.0006944)-

$\&($ WATDISP $*((1-$ EFFHARV)/EFFHARV $))$

QRECY = QHARV - WATLOSS

C

IF ( WATDISP .LT. 0.0) QHARV $=0.0$

C

C

C CALCULATE HARVEST SYSTEM REQUIREMENTS

C

$\mathrm{C}$

NHARV $=$ INT(QHARV/13.3+0.5)

QHARVB $=(($ QHARV- $($ WATDISP*(1-EFFHARV $))) *$ DENSITY $) /($ HCONC(IH) $)$

Page 5

FORTRAN77 V3.20 02/84

IF ( QHARVB .LT. WATPRO ) QHARV $=0.0$

HARVR $=($ HCONC $($ IALC $) *(D E N S I T Y * H C O N C(I H))) / 10000$

NHARVB = INT(QHARVB/3.8+0.5)

C

IF (NHARVB .LT. 1.0) NHARVB $=1.0$

$\mathrm{C}$

C CALCULATE HYDRAULIC DIAMETER, REYNOLDS NUMBER, HEAD LOSS

$\mathrm{C}$

$\mathrm{RH}=(\mathrm{CHAN} *$ DEPTH $) /($ CHAN $+(2 *$ DEPTH $))$

$\mathrm{RE}=\left(\mathrm{DENS} *\right.$ MIXVEL $\left.* \mathrm{RH}^{*} 2\right) /$ VISC

HL $=($ MIXVEL $* * 2) *\left(\right.$ ROUGH $\left.^{* *} 2\right) *($ LENGTH $*$ INT(WIDTH/CHAN +0.5$\left.)\right)$

C

$\& /(\mathrm{RH} * * 1.3333)$

C CALCULATE PUMPING HORSEPOWER REQUIREMENTS(M3/M)

C

UPHARVB $=1.04 *$ QHARVB

UPHARV $=.21 *$ QHARV

UPWATD $=1.55 *$ WATDISP

UPRECYC $=1.55 *$ QRECY 


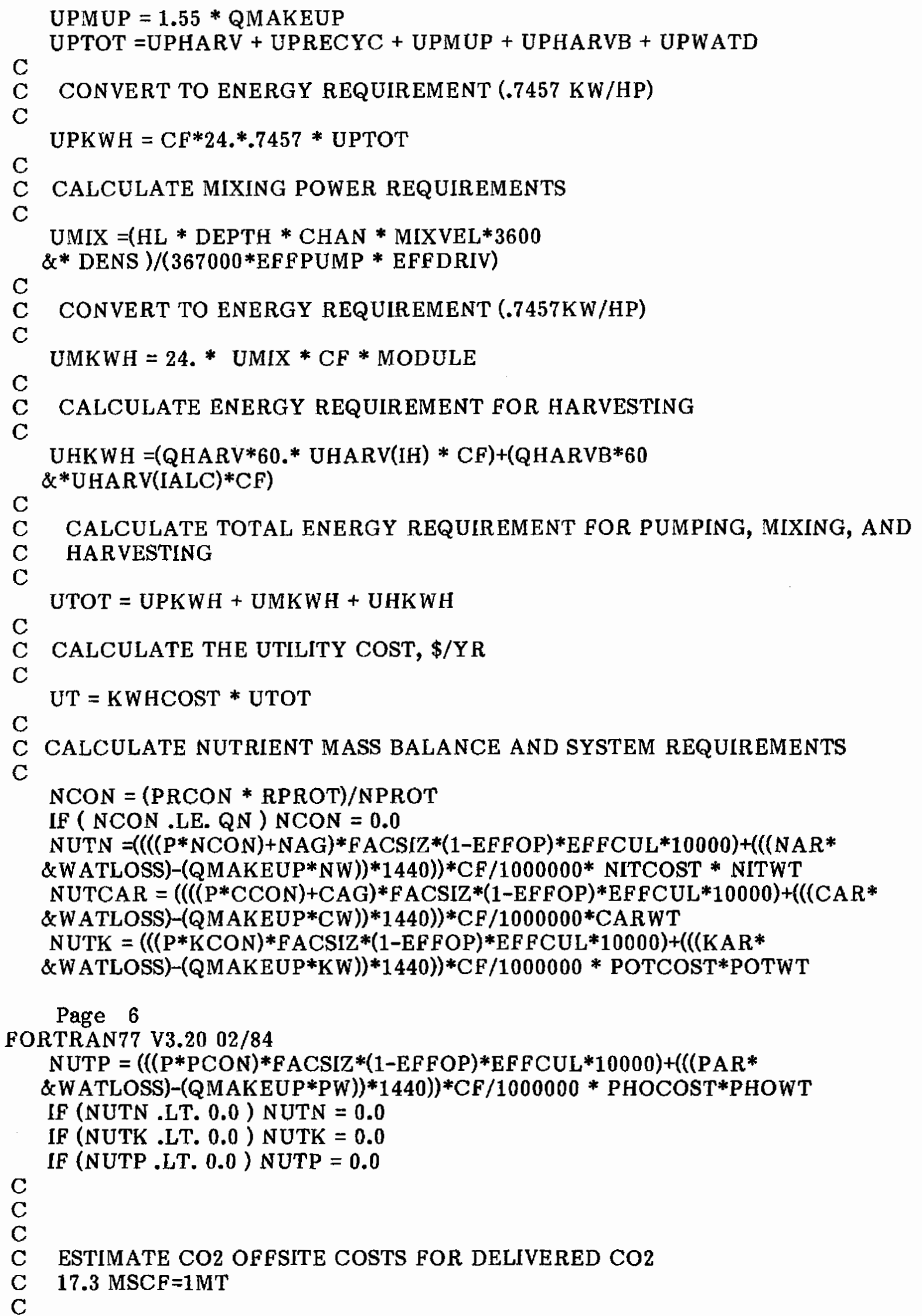




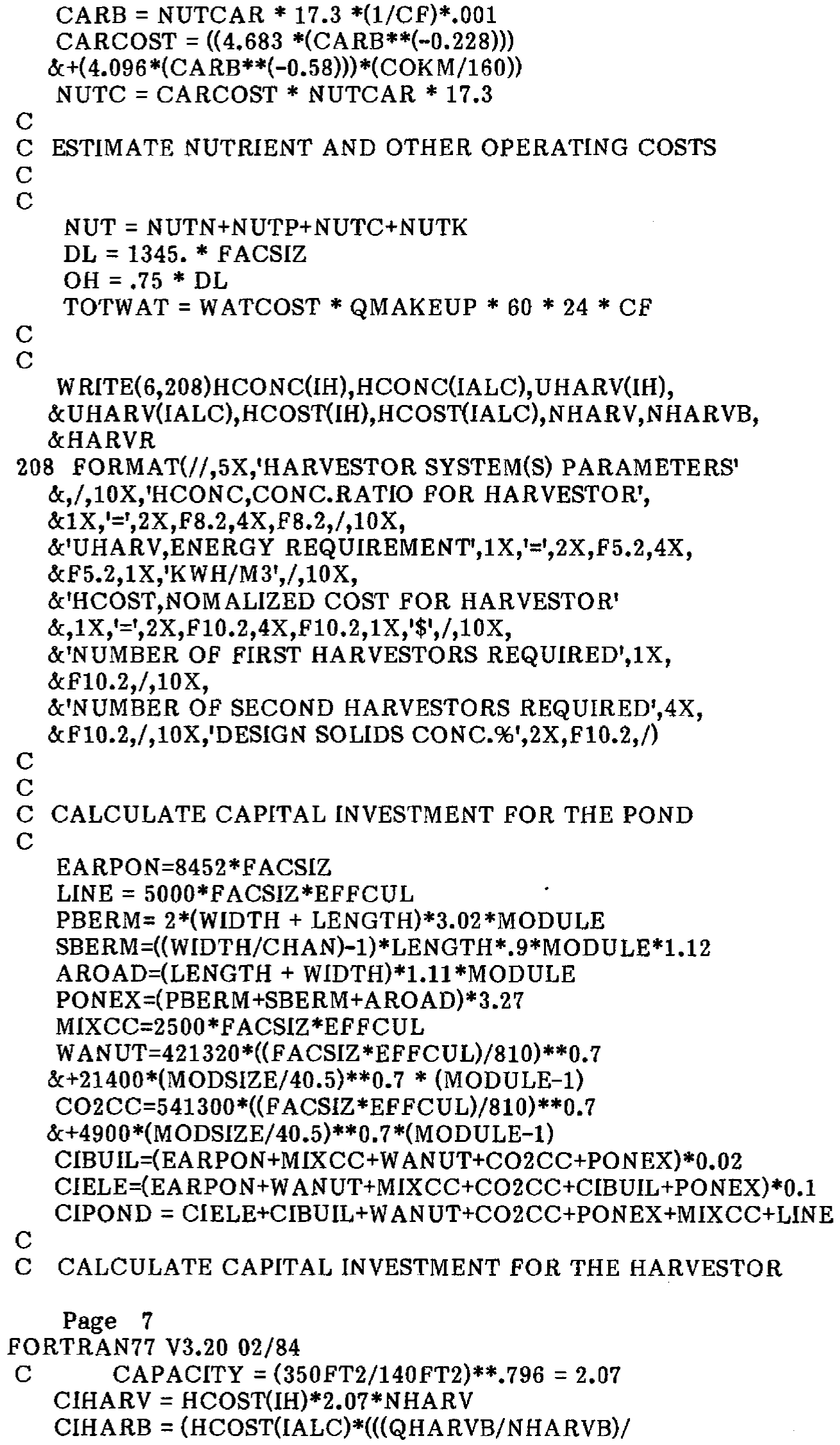


$\mathrm{C}$

C CALCULATE THE TOTAL DEPRECIABLE INVESTMENT

C

C

C

C

C

$\mathrm{C}$

C CALCULATE ENGINEERING, CONTINGENCIES, AND LAND COSTS

C

C

C CALCULATE DEPRECIABLE INVESTMENT

C

$\mathrm{DCI}=\mathrm{CI}$

$\mathrm{NDCI}=\mathrm{ENGFEE}+\mathrm{CONTG}+\mathrm{LCOST}+$ EARPON

W RITE $(6,201)$ LINE,MIXCC,PONEX, WANUT,CO2CC,CIBUIL,CIELE, \&CIPOND,CIHARV,CIHARB,DCI,EARPON,ENGFEE,CONTG,LCOST,NDCI

201 FORMAT(/,10X,'CAPITAL COST OF LINING, \$ ',E15.8,

$\& /, 10 X, '$ CAPITAL COST OF MIXING SYSTEM $\$, E 15.8$,

$\& /, 10 \mathrm{X}$, 'CAPITAL COST OF CULTURE SYSTEM \$',E15.8,

$\& /, 10 X,{ }^{\prime}$ CAPITAL COST OF WATER/NUTRIENT SYSTEM, $\$ 1$, E15.8,

$\& /, 10 X,{ }^{\prime}$ CAPITAL COST OF CARBON DIOXIDE SYSTEM, $\$, E 15.8$,

$\& /, 10 X, ' C A P T I A L$ COST OF BUILDINGS, \$',E15.8,

$\& /, 10 X,{ }^{2}$ A PTIAL COST OF ELECTRICAL SYSTEM, \$'E15.8,

$\& /, 10 X, ' C A P I T A L$ COST OF TOTAL CULTURE SYSTEM, $\$$ ',E15.8,

$\& /, 10 \mathrm{X}$, 'CAPITAL COST OF 1STHARVESTOR SYSTEM, $\$$ ',E15.8,

$\& /, 10 \mathrm{X}$, 'CAPITAL COST OF 2ND HARVESTOR SYSTEM, $\$$ 'E15.8,

$\& /, 10 X$, TOTAL DEPRECIABLE IN VESTMENT, $\$=1$, E15.8,

$\& / /, 10 \mathrm{X},{ }^{\prime}$ COST OF SITE PREP.AND SURVEY, $\$$ 'E15.8,

$\& /, 10 X,{ }^{\prime} E N G I N E E R I N G$ FEE, $\$=1$, E15.8,

$\& /, 10 \mathrm{X}$, 'CONTIGENCIES, $\$='$,E15.8,

$\& /, 10 \mathrm{X}$, 'LAND COST, $\$=+$, E15.8,

$\& /, 10 X$,'TOTAL NON-DEPRECIABLE INVESTMENT, $\$=+$,E15.8)

W RITE $(6,204)$ RE,QHARV,UPHARV,QHARVB,UPHARVB,QRECY, \&UPRECYC,WATDISP,UPW ATD,QMAKEUP,UPM UP,UPTOT,UPKWH, \&UMIX,UMKWH,UHKWH,UTOT

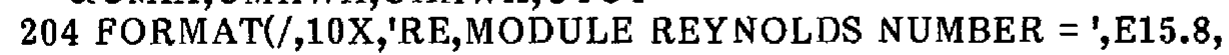

$\& /, 10 X, ' Q H A R V, M 3 / M I N=t, E 15.8,5 X, ' U P H A R V, H P,='$ 'E15.8,

$\& /, 10 X, ' Q H A R V B, M 3 / M I N=', E 15.8,5 X, '$ UPHARVB,HP, = ',E15.8, $\& /, 10 X, ' Q R E C Y, M 3 / M I N,=', E 15.8,5 X,{ }^{\prime} U P R E C Y C, H P,=', E 15.8$, $\& /, 10 X,{ }^{\prime}$ W ATDISP, M3/MIN, =',E15.8,5X,'UPWATD,HP, =',E15.8, $\& /, 10 X, ' Q M A K E U P, M 3 / M I N,={ }^{\dagger}, E 15.8,5 X, ' U P M U P, H P,='$ E15.8, $\& /, 10 X,{ }^{\prime}$ UPTOT,HP, $='$ 'E15.8,5X,'UPKW H,KWH, =',E15.8, $\& /, 10 \mathrm{X}, \mathrm{UM} I \mathrm{XX}, \mathrm{HP},=^{t}, \mathrm{E} 15.8,5 \mathrm{X}, \mathrm{UMKWH}, \mathrm{KWH},=^{\prime}, \mathrm{E} 15.8$, $\& /, 10 X, '$ 'UHKWH, KWH = ',E15.8,/,10X,'UTOT,KWH, =',E15.8) 
WRITE $(6,202)$ DL,OH,UT,CARB,CARCOST,NUTC,NUTN, \&NUTK,NUTP,NUT,TOTWAT,OPX,MNT,OP

Page 8

FORTRAN77 V3.20 02/84

202 FORMAT(//,10X,'DIRECT LABOR, $\$=1, E 15.8, /, 10 \mathrm{X}$, 'OVERHEAD,

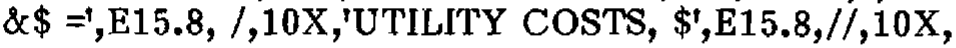
\&'CARBON REQUIRED',4X,E15.8,2X,'MMSCFD',/,10X, $\&^{\prime} \mathrm{AT} \mathrm{T}^{\prime}, 2 \mathrm{X}, \mathrm{E} 15.8,{ }^{\prime} \$$ PER MSCF',/, $\& 10 \mathrm{X}$, 'CARBON, $\$=1, \mathrm{E} 15.8, /, 10 \mathrm{X}$, \&'NITROGEN, $\$=$ ',E15.8,/,10X,'POTASSIUM, \$ =', $\&$ E15.8,/,10X,'PHOSPHORUS, $\$='$ 'E15.8,//, 10X, \&'TOTAL NUTRIENTS, $\$='$,E15.8,///,10X, \&'TOTAL WATER COSTS, $\$=', \mathrm{E} 15.8, / /, 10 \mathrm{X}$, \&'OTHER OPERATING COSTS, $\$=', \mathrm{E} 15.8, / /, 10 \mathrm{X}$, \&'MAINTENANCE COSTS, $\$=\prime$, E15.8, $/ /, 10 \mathrm{X}$, C \&"TOTAL OPERATING COSTS, $\$=', E 15.8, /)$

$\mathrm{C}$

C WRITE INPUT FILE FOR DOANE METHODOLOGY

C

$\mathrm{C}$

C

$499 \mathrm{ZMNT}=0.0$ W RITE $(8,500)(\mathrm{AA}(\mathrm{I}), \operatorname{ATEXT}(\mathrm{I}),[=60,86), \mathrm{DCI}, \mathrm{NDCI}, \mathrm{OP}, \mathrm{MNT}$, \&ANET,BBLS,CARBP,PROP

500 FORMAT(27(E15.8,4X,A60,/),E15.8,4X, \&'DCI, DEPRECIABLE CAPITAL INVESTMENT, \$',/,E15.8,4X, \&'NDCI, NON DEPRECIABLE CAPITAL INVESTMENT, \$',/,E15.8,4X, \&'OP, ANNUAL OPERATING COSTS, \$ $, /, \mathrm{E} 15.8,4 \mathrm{X}$, \&'MNT, ANNUAL MAINTENANCE COSTS, \$ ',/,E15.8,4X, \&'ANET, ANNUAL SYSTEM ALGAL YIELD,MT/YR ',/,E15.8,4X, \&'BBLS, ANNUAL SYSTEM LIPID YIELD,BBLS/YR ',/,E15.8,4X, \&'CARBP,CARBOHYDRATE YIELD MT/YR ',/, \&E15.8,4X,'PROTEIN YIELD, MT/YR',/)

STOP

END 


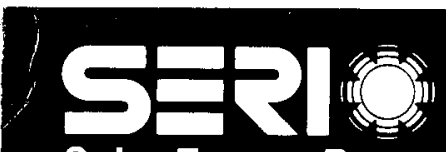

Solar Energy Research Institute

A Division of Midwest Research Institute

1617 Cole Boulevard

Goldèn: Colơrado 80401

Operated for the

U.S. Department of Energy

Contract No. DE-AC02-83CH10093 